Supporting Information

\title{
Diastereoselective synthesis of 2,3,4-trisubstituted tetrahydrofurans via thermally reactive 1,5-diene-tert-butyl carbonates.
}

\author{
Fabien Emmetiere ${ }^{1}$, Alexander J. Grenning ${ }^{1 *}$ \\ ${ }^{1}$ University of Florida, Department of Chemistry, Gainesville, FL, 32611 \\ *grenning@ufl.edu
}

Table of content

1. General experimental details --- 2

2. General procedures -------- 2

a. Knoevenagel adduct $\mathrm{S}_{\mathrm{N}} 2$ alkylation ---

i. Procedure A-1

ii. Procedure A-2

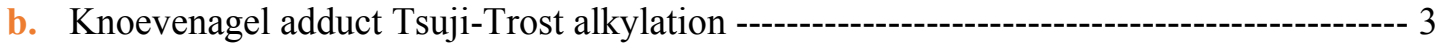

i. Procedure B-1

ii. Procedure B-2

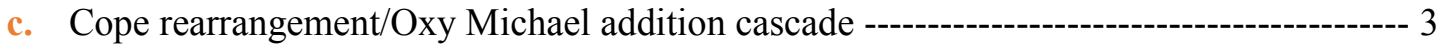

i. Procedure $\mathrm{C}-1$

ii. Procedure $\mathrm{C}-2$

d. Specific experimental procedures --- 4

i. Allylation/RCM sequence of iso-3a and 3a

ii. Ring-closing metathesis of $\mathbf{5 a} / \mathbf{5 b}$ and $\mathbf{3 c} / \mathbf{8 a}$

iii. Krapcho decarboxylation of $\mathbf{3 g}$

iv. Cross-metathesis of iso-3a and SI-6a

v. Oxidative esterification of SI-7a and 8a

vi. Retro-Oxy-Michael addition on 3a

vii. Detailed representative large-scale reaction

3. ${ }^{1} \mathrm{H},{ }^{13} \mathrm{C}$ NMR \& mass spectrometry data

4. Structural and Stereochemical assignment control experiments

a. Conversion and diastereoselectivity ratio for Scheme 4

b. Stereochemical outcome of the Cope rearrangement (3 and 4 position)

c. Stereochemical outcome of the Cope rearrangement/Oxy-Michael (2 position)

5. References

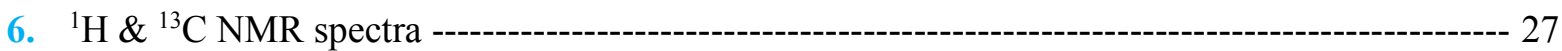




\section{General experimental details}

All commercial materials were used without further purification. Knoevenagel adducts were synthesized from modified reported literature procedures that are referenced along the way. All other synthetic protocols are outlined below. ${ }^{1} \mathrm{H}$ NMR and ${ }^{13} \mathrm{C}$ NMR spectra were recorded in $\mathrm{CDCl}_{3}$ using a $400 \mathrm{MHz}$ or $600 \mathrm{MHz}$ Varian VNMRS spectrometer (with $\mathrm{CHCl}_{3}$ residual peak as an internal standard) unless specified otherwise. All ${ }^{13} \mathrm{C}$ NMR spectra were recorded with complete proton decoupling. HRMS data were recorded on Agilent Time of Flight 6200 spectrometer. Reaction progress was monitored by thin-layer chromatography (TLC) and visualized by UV light, phosphomolybdic acid stain, and $\mathrm{KMnO}_{4}$ stain. Compounds were purified via silica gel column chromatography using Hexanes/Ethyl Acetate (Hex/EtOAc) solvent mixture. All reactions were carried out using anhydrous solvents obtained dried by passing through activated alumina columns. Notation: Hex/EtOAc X \% reads "mixture of $10 \%$ ethyl acetate (EtOAc) in hexanes (Hex)". Thermal reaction $\left(175^{\circ} \mathrm{C}\right.$ or above) were performed in Biotage ${ }^{\circledR}$ microwave reaction vial $2-5 \mathrm{~mL}$ or 10-20 mL sealed with Biotage ${ }^{\circledR}$ cap with septum. Microwaves vials were heated in Chemglass ${ }^{\circledR} 8$-place pie wedge for $2-5 \mathrm{~mL}$ microwave vials or Chemglass ${ }^{\circledR}$ 4-place pie wedge for $10-20 \mathrm{~mL}$ microwave vials. reaction time $(12 \mathrm{~h})$ was controlled by setting a timer using IKA® hotplates.

Deprotected by-products $\mathbf{4 e}, \mathbf{4 f}, \mathbf{4 g}$ and iso-4g weren't fully characterized. They were nonetheless unambiguously identified by ${ }^{1} \mathrm{H}$ and ${ }^{13} \mathrm{C}$ NMR and by comparison with their respective protected, fully characterized, precursors $E$-1e, $E$-1f, $E-\mathbf{1 g}$ and $Z$-1g (please see NMR spectra and reprints for details). All the other new molecules are fully documented.

\section{General procedures}

a. Knoevenagel adduct $\mathrm{S}_{\mathrm{N}} 2$ alkylation

i. Procedure A-1

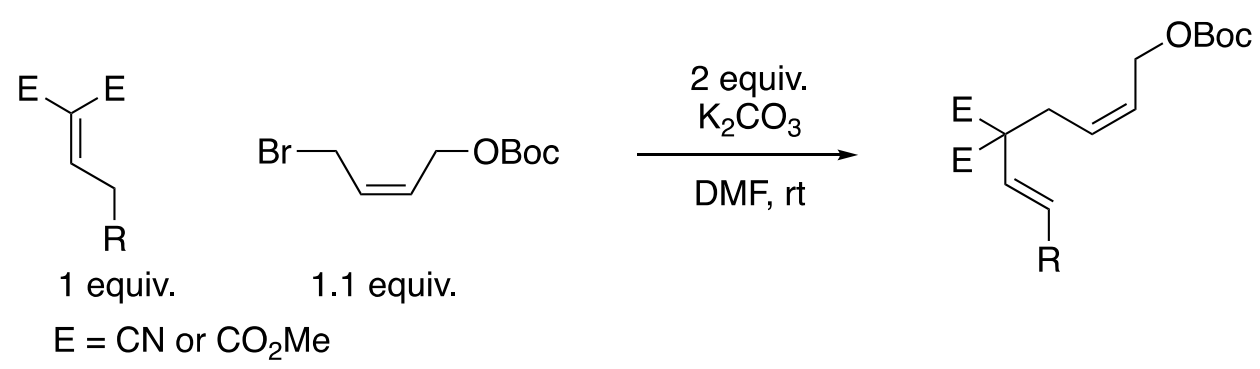

Pulverized $\mathrm{K}_{2} \mathrm{CO}_{3}$ (2 equiv.) was suspended in dry DMF in a flame-dried Schlenk flask under $\mathrm{N}_{2}$. A mixture of Knoevenagel adduct (1 equiv.) and (Z)-4-bromobut-2-en-1-yl tert-butyl carbonate ${ }^{1}$ (1.1 equiv.) in DMF (resulting in $0.1 \mathrm{M}$ concentration) was then added at room temperature. The reaction mixture was monitored by TLC. Once the reaction reached completion, the reaction mixture was quenched slowly using a $2 \mathrm{M}$ $\mathrm{HCl}_{(\mathrm{aq})}$ solution until no more effervescence was observed. The resulting aqueous mixture was extracted with EtOAc three times. The organic layers were then combined and washed sequentially with $2 \mathrm{M} \mathrm{HCl}_{(\mathrm{aq})}$ and brine before being dried over $\mathrm{Na}_{2} \mathrm{SO}_{4}$. The excess solvent was removed under reduced pressure and the crude mixture was purified via silica gel column chromatography (Hex/EtOAc).

\section{ii. Procedure A-2}

Same procedure as above but with $\mathrm{NaH}$ (1 equiv.) as base.

\section{b. Knoevenagel adduct Tsuji-Trost alkylation}


i. Procedure B-1<smiles>[R]CC=C(F)F</smiles>

1 equiv.

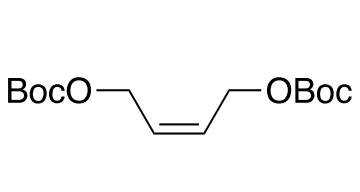

1.1 equiv.

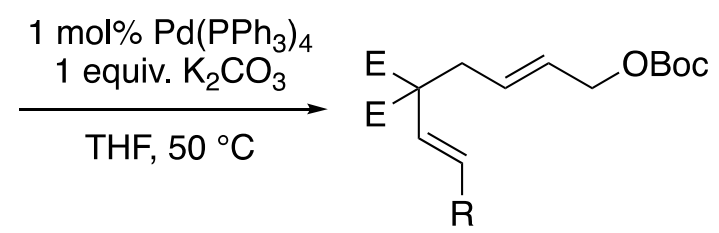

$$
\mathrm{E}=\mathrm{CN} \text { or } \mathrm{CO}_{2} \mathrm{Me}
$$

$\mathrm{Pd}\left(\mathrm{PPh}_{3}\right)_{4}(1 \mathrm{~mol} \%)$ and $\mathrm{K}_{2} \mathrm{CO}_{3}$ (1 equiv.) were charged in a flame-dried Schlenk flask under $\mathrm{N}_{2}$ and suspended in THF (0.1 M). bis-Boc protected (Z)-but-2-ene-1,4-diol ${ }^{2}$ (1.1 equiv.) and Knoevenagel adduct ( 1 equiv.) were added sequentially in this order. The reaction mixture was heated at $50{ }^{\circ} \mathrm{C}$ until completion (monitored by TLC). After completion, the reaction mixture was filtered through a short pad of silica eluted with EtOAc. The filtrate was concentrated down and the crude mixture was purified via silica column chromatography (Hex/EtOAc).

\section{ii. Procedure B-2}<smiles>[R]CC=C(F)F</smiles>

1 equiv.

$$
\mathrm{E}=\mathrm{CN} \text { or } \mathrm{CO}_{2} \mathrm{Me}
$$
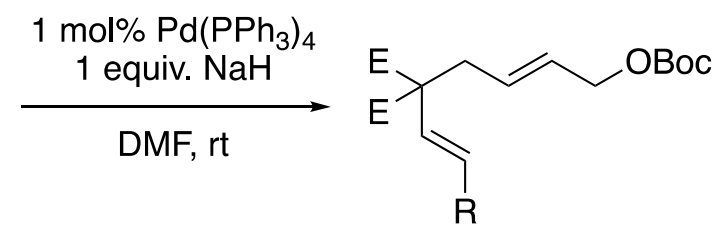

$\mathrm{Pd}\left(\mathrm{PPh}_{3}\right)_{4}(1 \mathrm{~mol} \%)$ and $\mathrm{NaH}$ (1 equiv.) were charged in a flame-dried Schlenk flask under $\mathrm{N}_{2}$ and suspended in DMF (0.1 M). bis-Boc protected (Z)-but-2-ene-1,4-diol ${ }^{2}$ (1.1 equiv.) and Knoevenagel adduct (1 equiv.) were added sequentially in this order. The reaction mixture was stirred at room temperature until completion (monitored by TLC). Once the reaction reached completion, the reaction mixture was quenched slowly using a $2 \mathrm{M} \mathrm{HCl}_{(\mathrm{aq})}$ solution until no more effervescence was observed. The resulting aqueous mixture was extracted with EtOAc three times. The organic layers were then combined and washed sequentially with $2 \mathrm{M} \mathrm{HCl}_{(\mathrm{aq})}$ and brine before being dried over $\mathrm{Na}_{2} \mathrm{SO}_{4}$. The excess solvent was removed under reduced pressure and the crude mixture was purified via silica gel column chromatography (Hex/EtOAc).

\section{c. Cope rearrangement/Oxy Michael addition cascade}

\section{i. Procedure $\mathrm{C}-1$}<smiles>[R]C=CC(F)(F)CC=CCOC(C)(C)C</smiles>

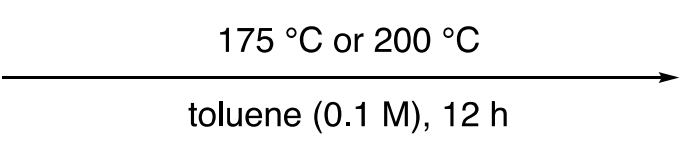<smiles>[2H][C@@H]1COC(C(F)F)[C@H]1C=C</smiles>

1 equiv. $\mathrm{E}=\mathrm{CN}$ or $\mathrm{CO}_{2} \mathrm{Me}$ 
Alkylated Knoevenagel precursor was charged in a flame-dried microwave pressured vial (see specifics in the General Experimental Section) under $\mathrm{N}_{2}$. Toluene is added $(0.1 \mathrm{M})$ and the pressured flask was sealed. The reaction mixture was heated at $175^{\circ} \mathrm{C}$ for $12 \mathrm{~h}$ (see specifics in the General Experimental Section). After $12 \mathrm{~h}$ reaction, the flask was cooled down to room temperature and toluene was removed under reduced pressure. The resulting crude mixture was purified via silica gel column chromatography (Hex/EtOAc).

\section{ii. Procedure C-2}

Same as Procedure C-1 but at $200{ }^{\circ} \mathrm{C}$.

\section{d. Specific experimental procedures}

i. Allylation/RCM sequence of iso-3a and 3a

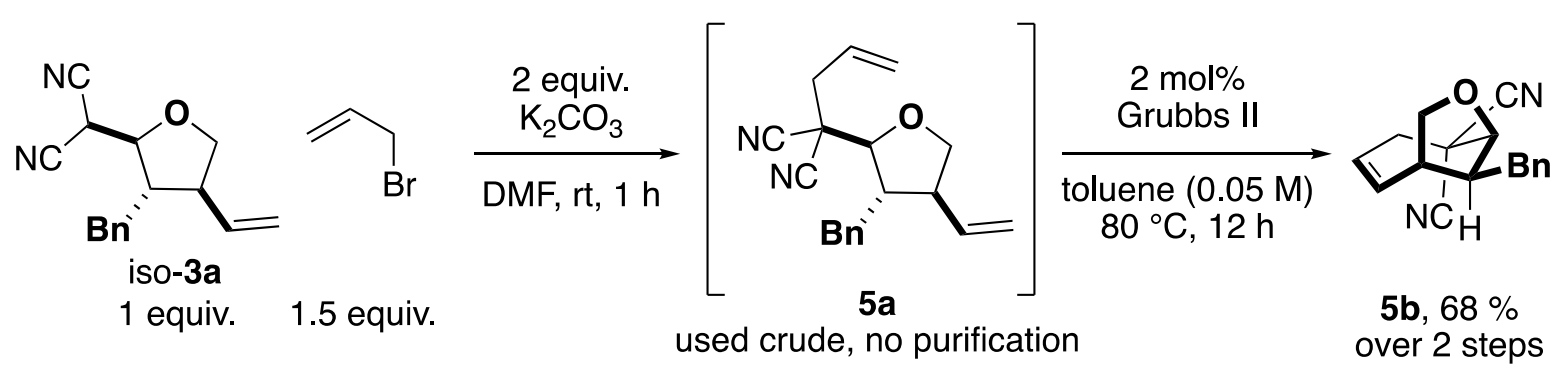

$\mathrm{K}_{2} \mathrm{CO}_{3}$ (2 equiv., $22 \mathrm{mg}, 0.16 \mathrm{mmol}$ ) was charged in a flame-dried Schlenk flask under $\mathrm{N}_{2}$ and suspended in DMF $(0.5 \mathrm{~mL})$. iso-3a (1 equiv., $20 \mathrm{mg}, 0.079 \mathrm{mmol}$ ) in $0.5 \mathrm{~mL}$ DMF was then added immediately followed by allyl bromide ( 1.5 equiv., $10 \mu \mathrm{L}, 0.12 \mathrm{mmol}$ ) at room temperature. The reaction was stirred for $1 \mathrm{~h}$ at room temperature and was then quenched slowly using a $2 \mathrm{M} \mathrm{HCl}_{(\mathrm{aq})}(3 \mathrm{~mL})$ solution until no more effervescence was observed. The resulting aqueous mixture was extracted with EtOAc three times $(3 \times 10$ $\mathrm{mL})$. The organic layers were then combined and washed sequentially with $2 \mathrm{M} \mathrm{HCl}_{(\mathrm{aq})}(10 \mathrm{~mL})$ and brine $(10 \mathrm{~mL})$ before being dried over $\mathrm{Na}_{2} \mathrm{SO}_{4}$. The excess solvent was removed under reduced pressure and $\mathbf{5 a}$ was used in the next step without further purification (8:1 d.r. from crude NMR).

Note: Crude intermediate 5a wasn't fully characterized (no HRMS) but crude ${ }^{1} \mathrm{H}$ and ${ }^{13} \mathrm{C}$ NMR data analysis can be found below:

${ }^{1}$ H NMR (400 MHz, Chloroform- $d$ ) $\delta 7.31(\mathrm{t}, J=7.3 \mathrm{~Hz}, 2 \mathrm{H}), 7.22(\mathrm{t}, J=9.4 \mathrm{~Hz}, 3 \mathrm{H}), 5.91$ (ddt, $J=17.3$, $10.4,7.3 \mathrm{~Hz}, 1 \mathrm{H}), 5.60(\mathrm{dt}, J=17.9,9.2 \mathrm{~Hz}, 1 \mathrm{H}), 5.49-5.33(\mathrm{~m}, 2 \mathrm{H}), 5.09-4.99(\mathrm{~m}, 2 \mathrm{H}), 3.95(\mathrm{dt}, J=$ $8.0,4.6 \mathrm{~Hz}, 2 \mathrm{H}), 3.82(\mathrm{t}, J=9.2 \mathrm{~Hz}, 1 \mathrm{H}), 3.11(\mathrm{dd}, J=14.0,4.6 \mathrm{~Hz}, 1 \mathrm{H}), 2.93(\mathrm{dd}, J=14.1,7.3 \mathrm{~Hz}, 1 \mathrm{H})$, $2.86-2.76(\mathrm{~m}, 2 \mathrm{H}), 2.64-2.51(\mathrm{~m}, 2 \mathrm{H})$.

${ }^{13}$ C NMR $\left(101 \mathrm{MHz}, \mathrm{CDCl}_{3}\right) \delta 137.3,135.4,130.1,128.7,127.0,123.4,118.3,114.6,114.1,83.6,77.2$, $73.3,50.3,49.6,42.9,39.6,37.3$.

Grubbs $2^{\text {nd }}$ generation catalyst $(2 \mathrm{~mol} \%, 1 \mathrm{mg}, 0.0014 \mathrm{mmol}$ ) was charged in a flame-dried Schlenk flask under $\mathrm{N}_{2}$ and dissolved in toluene $(0.4 \mathrm{~mL})$. Crude $5 \mathbf{a}$ in $1 \mathrm{~mL}$ toluene $(0.05 \mathrm{M}$ final concentration) was added at room temperature. The reaction mixture was then stirred at $80^{\circ} \mathrm{C}$ for $12 \mathrm{~h}$. After $12 \mathrm{~h}$, the reaction mixture was cooled down to room temperature and the solvent was removed under reduced pressure. The crude residue was purified via silica gel column chromatography (Hex/EtOAc $10 \%$ ) yielding bicyclic $\mathbf{5 b}$ as a colorless oil (13.5 mg, $68 \%$ over 2 steps, single diastereomer).

${ }^{1}$ H NMR (600 MHz, Chloroform- $d$ ) $\delta 7.35(\mathrm{t}, J=7.6 \mathrm{~Hz}, 2 \mathrm{H}), 7.30-7.26(\mathrm{~m}, 1 \mathrm{H}), 7.20(\mathrm{~d}, J=7.3 \mathrm{~Hz}$, $2 \mathrm{H}), 6.28$ (ddd, $J=11.5,8.7,3.1 \mathrm{~Hz}, 1 \mathrm{H}), 5.64(\mathrm{ddd}, J=11.7,8.6,3.5 \mathrm{~Hz}, 1 \mathrm{H}), 4.63(\mathrm{~s}, 1 \mathrm{H}), 4.39$ (dd, $J=$ $8.7,6.6 \mathrm{~Hz}, 1 \mathrm{H}), 4.17(\mathrm{dd}, J=8.7,1.3 \mathrm{~Hz}, 1 \mathrm{H}), 3.10(\mathrm{dt}, J=15.9,3.3 \mathrm{~Hz}, 1 \mathrm{H}), 2.79(\mathrm{q}, J=9.0,8.5 \mathrm{~Hz}$, $1 \mathrm{H}), 2.74(\mathrm{q}, J=5.9 \mathrm{~Hz}, 2 \mathrm{H}), 2.71(\mathrm{~d}, J=5.9 \mathrm{~Hz}, 1 \mathrm{H}), 2.70-2.66(\mathrm{~m}, 1 \mathrm{H})$.

${ }^{13}$ C NMR $\left(151 \mathrm{MHz}, \mathrm{CDCl}_{3}\right) \delta 140.5,138.0,129.1,129.0,127.2,122.7,116.0,114.4,85.7,75.4,50.0$, $41.1,38.9,38.1,31.2$. 
HRMS (ESI - TOF) m/z: Calcd for $\mathrm{C}_{17} \mathrm{H}_{15} \mathrm{~N}_{2} \mathrm{O}[\mathrm{M}-\mathrm{H}]^{-}$263.1179, found 263.1183.

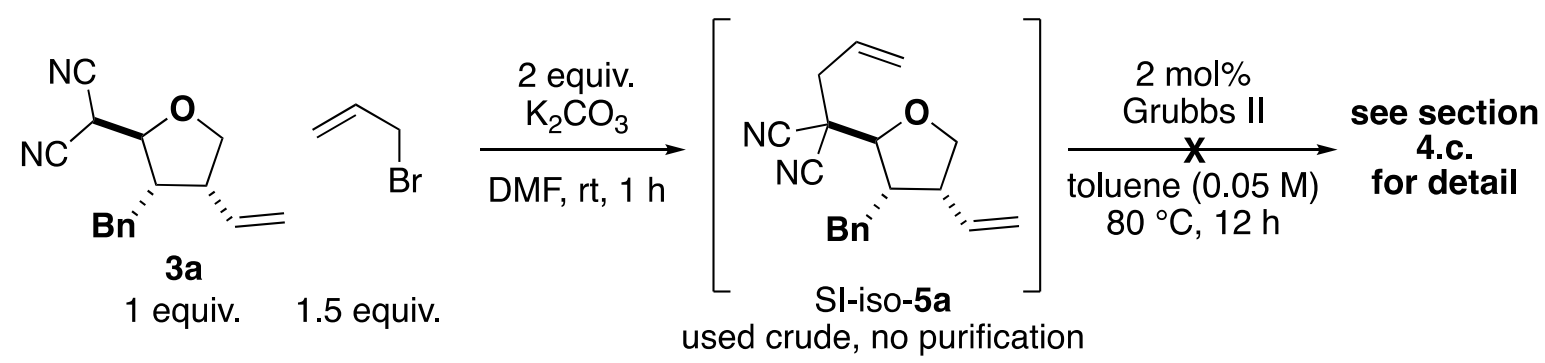

$\mathrm{K}_{2} \mathrm{CO}_{3}$ (2 equiv., $22 \mathrm{mg}, 0.16 \mathrm{mmol}$ ) was charged in a flame-dried Schlenk flask under $\mathrm{N}_{2}$ and suspended in DMF (0.5 mL). 3a (1 equiv., $19 \mathrm{mg}, 0.078 \mathrm{mmol}$ ) in $0.5 \mathrm{~mL}$ DMF was then added immediately followed by allyl bromide ( 1.5 equiv., $10 \mu \mathrm{L}, 0.12 \mathrm{mmol}$ ) at room temperature. The reaction was stirred for $1 \mathrm{~h}$ at room temperature and was then quenched slowly using a $2 \mathrm{M} \mathrm{HCl}_{(\mathrm{aq})}(3 \mathrm{~mL})$ solution until no more effervescence was observed. The resulting aqueous mixture was extracted with EtOAc three times $(3 \times 10$ $\mathrm{mL})$. The organic layers were then combined and washed sequentially with $2 \mathrm{M} \mathrm{HCl}_{(\mathrm{aq})}(10 \mathrm{~mL})$ and brine $(10 \mathrm{~mL})$ before being dried over $\mathrm{Na}_{2} \mathrm{SO}_{4}$. The excess solvent was removed under reduced pressure and SIiso-5a was used in the next step without further purification (5:1 d.r. from crude NMR).

Grubbs $2^{\text {nd }}$ generation catalyst $(2 \mathrm{~mol} \%, 1 \mathrm{mg}, 0.0014 \mathrm{mmol})$ was charged in a flame-dried Schlenk flask under $\mathrm{N}_{2}$ and dissolved in toluene $(0.4 \mathrm{~mL})$. Crude SI-iso-5a in $1 \mathrm{~mL}$ toluene (0.05 M final concentration) was added at room temperature. The reaction mixture was then stirred at $80{ }^{\circ} \mathrm{C}$ for $12 \mathrm{~h}$. After $12 \mathrm{~h}$, the reaction mixture was cooled down to room temperature and the solvent was removed under reduced pressure. This reaction was performed as a control experiment, please see Section 4.c.

Note: Crude intermediate SI-iso-5a wasn't fully characterized (no HRMS) but crude ${ }^{1} \mathrm{H}$ and ${ }^{13} \mathrm{C}$ NMR data analysis can be found below:

${ }^{1}$ H NMR $(600 \mathrm{MHz}$, Chloroform- $d$ ) $\delta 7.32(\mathrm{t}, J=7.5 \mathrm{~Hz}, 2 \mathrm{H}), 7.26-7.20(\mathrm{~m}, 3 \mathrm{H}), 5.80$ (tq, $J=16.7,8.4$, $7.3 \mathrm{~Hz}, 2 \mathrm{H}), 5.37(\mathrm{~d}, J=10.2 \mathrm{~Hz}, 1 \mathrm{H}), 5.33(\mathrm{~d}, J=17.0 \mathrm{~Hz}, 1 \mathrm{H}), 5.26(\mathrm{~d}, J=10.3 \mathrm{~Hz}, 1 \mathrm{H}), 5.16(\mathrm{~d}, J=$ $17.2 \mathrm{~Hz}, 1 \mathrm{H}), 4.19(\mathrm{dd}, J=8.6,5.9 \mathrm{~Hz}, 1 \mathrm{H}), 3.96(\mathrm{~d}, J=4.6 \mathrm{~Hz}, 1 \mathrm{H}), 3.86(\mathrm{dd}, J=8.6,6.1 \mathrm{~Hz}, 1 \mathrm{H}), 3.21$ $(\mathrm{p}, J=6.7 \mathrm{~Hz}, 1 \mathrm{H}), 2.93-2.87(\mathrm{~m}, 1 \mathrm{H}), 2.84-2.77(\mathrm{~m}, 2 \mathrm{H}), 2.58(\mathrm{dd}, J=14.0,7.0 \mathrm{~Hz}, 1 \mathrm{H}), 2.45(\mathrm{dd}, J=$ 13.9, 7.6 Hz, 1H).

${ }^{13}$ C NMR $\left(151 \mathrm{MHz}, \mathrm{CDCl}_{3}\right) \delta 138.6,134.0,129.3,128.9,128.6,126.9,123.2,119.0,114.5,113.8,83.7$, $77.2,73.5,48.6,46.3,43.2,39.4,34.8$.

ii. Ring-closing metathesis of $\mathbf{3 c} / \mathbf{8 a}$
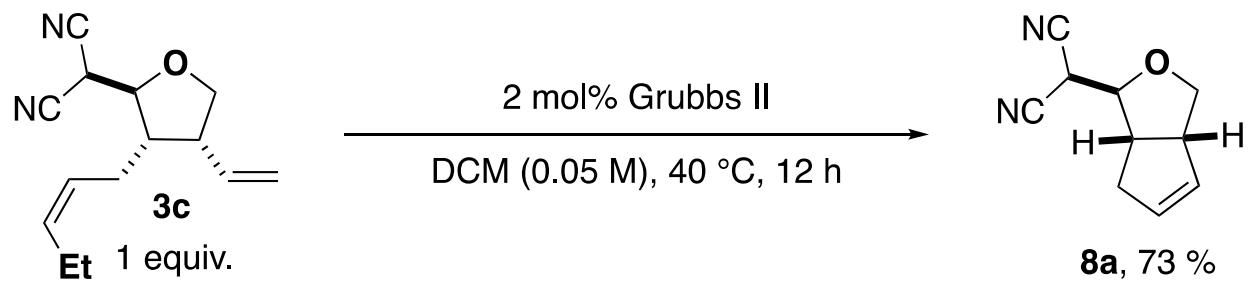

$8 a, 73 \%$

Grubbs $2^{\text {nd }}$ generation catalyst ( $2 \mathrm{~mol} \%, 1.5 \mathrm{mg}, 0.0017 \mathrm{mmol}$ ) was charged in a flame-dried Schlenk flask under $\mathrm{N}_{2}$ and dissolved in DCM $(0.7 \mathrm{~mL})$. 3c (1 equiv., $\left.20 \mathrm{mg}, 0.086 \mathrm{mmol}\right)$ in $1 \mathrm{~mL} \mathrm{DCM}(0.05 \mathrm{M}$ final concentration) was added at room temperature. The reaction mixture was then stirred at $40{ }^{\circ} \mathrm{C}$ for $12 \mathrm{~h}$. After $12 \mathrm{~h}$, the reaction mixture was cooled down to room temperature and the solvent was removed under reduced pressure. The crude residue was purified via silica gel column chromatography (Pentanes/Et ${ }_{2} \mathrm{O} 20$ \%) yielding bicyclic 8a as a colorless oil (11 mg, $73 \%$, single diastereomer). 
${ }^{1}$ H NMR (600 MHz, Chloroform- $d$ ) $\delta 5.74(\mathrm{dq}, J=4.7,2.2 \mathrm{~Hz}, 1 \mathrm{H}), 5.66$ (dq, $\left.J=5.7,2.1 \mathrm{~Hz}, 1 \mathrm{H}\right), 4.28$ (dd, $J=8.9,7.3 \mathrm{~Hz}, 1 \mathrm{H}), 3.96(\mathrm{dd}, J=6.3,5.2 \mathrm{~Hz}, 1 \mathrm{H}), 3.92$ (d, $J=5.2 \mathrm{~Hz}, 1 \mathrm{H}), 3.83$ (dd, $J=8.9,3.6 \mathrm{~Hz}$, $1 \mathrm{H}), 3.63$ (dddq, $J=9.8,5.9,3.9,2.2 \mathrm{~Hz}, 1 \mathrm{H}), 2.91$ (tdd, $J=8.0,6.3,1.6 \mathrm{~Hz}, 1 \mathrm{H}$ ), 2.75 (ddq, $J=17.2,7.8$, $2.4 \mathrm{~Hz}, 1 \mathrm{H}), 2.40(\mathrm{dp}, J=17.2,2.1 \mathrm{~Hz}, 1 \mathrm{H})$.

${ }^{13}$ C NMR $\left(151 \mathrm{MHz}, \mathrm{CDCl}_{3}\right) \delta 132.2,130.4,111.3,111.0,84.2,77.2,74.3,52.6,46.4,37.2,28.3$.

HRMS (ESI - TOF) m/z: Calcd for $\mathrm{C}_{10} \mathrm{H}_{9} \mathrm{~N}_{2} \mathrm{O}[\mathrm{M}-\mathrm{H}]^{-}$173.0720, found 173.0715.

iii. Krapcho decarboxylation of $\mathbf{3 g}$

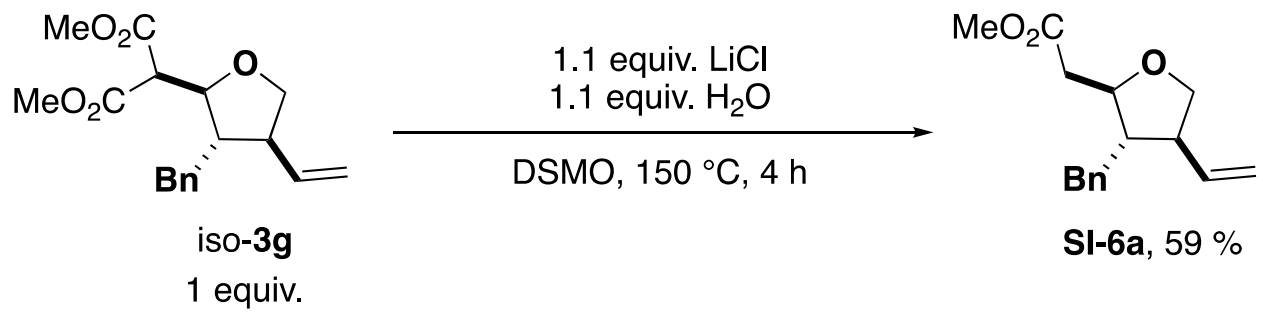

iso-3g (1 equiv., $35 \mathrm{mg}, 0.11 \mathrm{mmol}$ ) was dissolved in $0.5 \mathrm{~mL}$ DMSO is a microwave pressured flask. $\mathrm{LiCl}$ (1.1 equiv. $5 \mathrm{mg}, 0.12 \mathrm{mmol})$ and $\mathrm{H}_{2} \mathrm{O}(\sim 1.1$ equiv., $7 \mu \mathrm{L}, \sim 0.12 \mathrm{mmol})$ were added and the flask was sealed. The reaction mixture was heated at $150{ }^{\circ} \mathrm{C}$ for $4 \mathrm{~h}$. After completion, the reaction mixture was cooled down to room temperature and diluted with a $2 \mathrm{M} \mathrm{HCl}_{(\mathrm{aq})}(5 \mathrm{~mL})$ solution. The resulting aqueous mixture was extracted with EtOAc three times $(3 \times 5 \mathrm{~mL})$. The organic layers were then combined and washed sequentially with $2 \mathrm{M} \mathrm{HCl}_{(\mathrm{aq})}(10 \mathrm{~mL})$ and brine $(10 \mathrm{~mL})$ before being dried over $\mathrm{Na}_{2} \mathrm{SO}_{4}$. The excess solvent was removed under reduced pressure and the crude residue was purified via silica gel column chromatography (Hex/EtOAc $10 \%$ ). SI-6a was obtained as a colorless oil (17 mg, 59 \%, 7:1 d.r., major reported below).

${ }^{1}$ H NMR (400 MHz, Chloroform- $d$ ) $\delta 7.31-7.26(\mathrm{~m}, 3 \mathrm{H}), 7.23-7.18(\mathrm{~m}, 1 \mathrm{H}), 7.18-7.13(\mathrm{~m}, 2 \mathrm{H}), 5.72$ - $5.59(\mathrm{~m}, 1 \mathrm{H}), 5.15-5.00(\mathrm{~m}, 2 \mathrm{H}), 4.17-4.05(\mathrm{~m}, 1 \mathrm{H}), 4.01-3.91(\mathrm{~m}, 1 \mathrm{H}), 3.70-3.57(\mathrm{~m}, 3 \mathrm{H}), 2.94$ $(\mathrm{dt}, J=13.6,5.5 \mathrm{~Hz}, 1 \mathrm{H}), 2.66-2.52(\mathrm{~m}, 2 \mathrm{H}), 2.22(\mathrm{dd}, J=15.3,8.9 \mathrm{~Hz}, 1 \mathrm{H}), 2.11-2.06(\mathrm{~m}, 1 \mathrm{H}), 1.94$ (qt, $J=8.9,3.7 \mathrm{~Hz}, 1 \mathrm{H}$ ).

${ }^{13}$ C NMR $\left(101 \mathrm{MHz}, \mathrm{CDCl}_{3}\right) \delta 171.8,139.4,137.6,129.1,129.1,128.6,126.5,117.0,80.9,77.2,71.7$, 52.0, 51.8, 51.0, 40.0, 37.4.

HRMS (ESI - TOF) m/z: Calcd for $\mathrm{C}_{16} \mathrm{H}_{20} \mathrm{NaO}_{3}[\mathrm{M}+\mathrm{Na}]^{+} 283.1305$, found 283.1293.

iv. Cross-metathesis of iso-3a and SI-6a
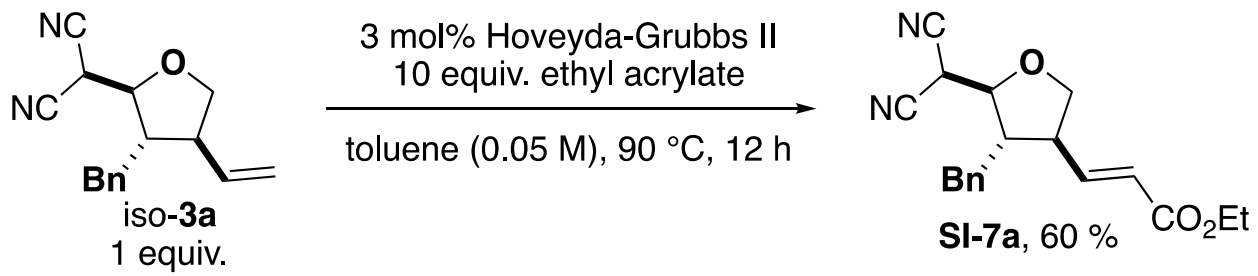

Hoveyda-Grubbs $2^{\text {nd }}$ generation catalyst $(3 \mathrm{~mol} \%, 1.5 \mathrm{mg}, 0.0024 \mathrm{mmol}$ ) was charged in a flame-dried Schlenk flaks under $\mathrm{N}_{2}$ and dissolved in $1 \mathrm{~mL}$ toluene. iso-3a (1 equiv., $20 \mathrm{mg}, 0.08 \mathrm{mmol}$ ) was added in $0.5 \mathrm{~mL}$ toluene $(0.05 \mathrm{M}$ final concentration) followed by ethyl acrylate (10 equiv., $84 \mu \mathrm{L}, 0.8 \mathrm{mmol})$. The reaction flask was sealed and heated at $90{ }^{\circ} \mathrm{C}$ for $12 \mathrm{~h}$. After $12 \mathrm{~h}$, the reaction mixture was cooled down to room temperature and the solvent was removed under reduced pressure. The crude residue was purified via 
silica gel column chromatography (Hex/EtOAc $20 \%$ ) yielding SI-7a as a yellowish oil (15 mg, 60 \%, 6:1 d.r., major reported below).

${ }^{1}$ H NMR (600 MHz, Chloroform- $\left.d\right) \delta 7.37(\mathrm{t}, J=7.5 \mathrm{~Hz}, 2 \mathrm{H}), 7.32-7.28(\mathrm{~m}, 1 \mathrm{H}), 7.17(\mathrm{~d}, J=6.9 \mathrm{~Hz}$, 2H), 6.79 (dd, $J=15.6,9.0 \mathrm{~Hz}, 1 \mathrm{H}), 5.94$ (d, $J=15.5 \mathrm{~Hz}, 1 \mathrm{H}), 4.22$ (q, $J=7.2 \mathrm{~Hz}, 2 \mathrm{H}), 4.14$ (dd, $J=8.9$, $7.7 \mathrm{~Hz}, 1 \mathrm{H}), 4.09(\mathrm{dd}, J=8.5,2.7 \mathrm{~Hz}, 1 \mathrm{H}), 3.91(\mathrm{dd}, J=9.9,8.9 \mathrm{~Hz}, 1 \mathrm{H}), 3.12(\mathrm{dd}, J=13.7,4.9 \mathrm{~Hz}, 1 \mathrm{H})$, $2.96-2.89(\mathrm{~m}, 1 \mathrm{H}), 2.73(\mathrm{~d}, J=2.6 \mathrm{~Hz}, 1 \mathrm{H}), 2.57(\mathrm{dd}, J=13.7,10.1 \mathrm{~Hz}, 1 \mathrm{H}), 2.41$ (tdd, $J=10.0,8.5,5.0$ $\mathrm{Hz}, 1 \mathrm{H}), 1.31(\mathrm{t}, J=7.1 \mathrm{~Hz}, 3 \mathrm{H})$.

${ }^{13} \mathrm{C}$ NMR $\left(151 \mathrm{MHz}, \mathrm{CDCl}_{3}\right) \delta 165.6,143.6,137.4,129.6,128.6,127.9,125.2,111.3,110.8,82.0,72.9$, $60.9,50.4,49.3,36.9,28.6,14.4$.

HRMS (ESI - TOF) m/z: Calcd for $\mathrm{C}_{19} \mathrm{H}_{20} \mathrm{~N}_{2} \mathrm{NaO}_{3}[\mathrm{M}+\mathrm{Na}]^{+} 347.1366$, found 347.1375.

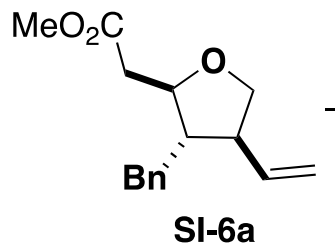

1 equiv.

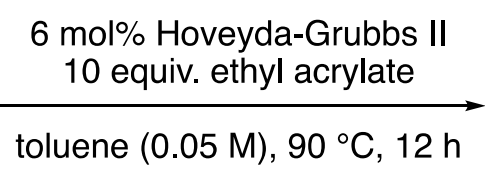

toluene $(0.05 \mathrm{M}), 90^{\circ} \mathrm{C}, 12 \mathrm{~h}$

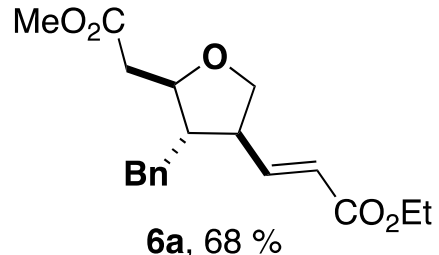

$6 a, 68 \%$

Hoveyda-Grubbs $2^{\text {nd }}$ generation catalyst $(6 \mathrm{~mol} \%, 2 \mathrm{mg}, 0.0029 \mathrm{mmol})$ was charged in a flame-dried Schlenk flaks under $\mathrm{N}_{2}$ and dissolved in $0.5 \mathrm{~mL}$ toluene. SI-6a (1 equiv., $15 \mathrm{mg}, 0.057 \mathrm{mmol}$ ) was added in $0.5 \mathrm{~mL}$ toluene $(0.05 \mathrm{M}$ final concentration) followed by ethyl acrylate (10 equiv., $80 \mu \mathrm{L}, 0.57 \mathrm{mmol})$. The reaction flask was sealed and heated at $90{ }^{\circ} \mathrm{C}$ for $12 \mathrm{~h}$. After $12 \mathrm{~h}$, the reaction mixture was cooled down to room temperature and the solvent was removed under reduced pressure. The crude residue was purified via silica gel column chromatography (Hex/EtOAc $15 \%$ ) yielding 6a as a yellow oil (13.5 mg, 68 $\%, 7: 1$ d.r., major reported below).

${ }^{1}$ H NMR (400 MHz, Chloroform- $d$ ) $\delta 7.30-7.27(\mathrm{~m}, 2 \mathrm{H}), 7.22-7.16(\mathrm{~m}, 1 \mathrm{H}), 7.16-7.11(\mathrm{~m}, 2 \mathrm{H}), 6.72$ $(\mathrm{dd}, J=15.6,9.2 \mathrm{~Hz}, 1 \mathrm{H}), 5.73(\mathrm{dd}, J=15.6,0.9 \mathrm{~Hz}, 1 \mathrm{H}), 4.17(\mathrm{q}, J=7.1 \mathrm{~Hz}, 2 \mathrm{H}), 4.14-4.07(\mathrm{~m}, 1 \mathrm{H})$, $3.96(\mathrm{dd}, J=8.9,7.6 \mathrm{~Hz}, 1 \mathrm{H}), 3.70-3.65(\mathrm{~m}, 2 \mathrm{H}), 3.65(\mathrm{~s}, 3 \mathrm{H}), 2.85(\mathrm{dd}, J=13.7,6.1 \mathrm{~Hz}, 1 \mathrm{H}), 2.76(\mathrm{tt}, J$ $=8.6,0.9 \mathrm{~Hz}, 1 \mathrm{H}), 2.66(\mathrm{dd}, J=13.8,8.2 \mathrm{~Hz}, 1 \mathrm{H}), 2.29(\mathrm{dd}, J=15.4,8.7 \mathrm{~Hz}, 1 \mathrm{H}), 2.15(\mathrm{dd}, J=15.4,3.5$ $\mathrm{Hz}, 1 \mathrm{H}), 2.08(\mathrm{qd}, J=8.4,6.2 \mathrm{~Hz}, 1 \mathrm{H}), 1.28(\mathrm{t}, J=7.1 \mathrm{~Hz}, 3 \mathrm{H})$.

${ }^{13}$ C NMR $\left(101 \mathrm{MHz}, \mathrm{CDCl}_{3}\right) \delta 171.6,166.2,147.1,138.7,129.1,128.8,126.8,122.9,81.0,71.2,60.5$, 52.0, 51.9, 49.2, 39.7, 37.6, 14.4.

HRMS (ESI - TOF) m/z: Calcd for $\mathrm{C}_{19} \mathrm{H}_{24} \mathrm{NaO}_{5}[\mathrm{M}+\mathrm{Na}]^{+} 355.1516$, found 355.1503.

\section{v. Oxidative esterification of SI-7a and 8a}

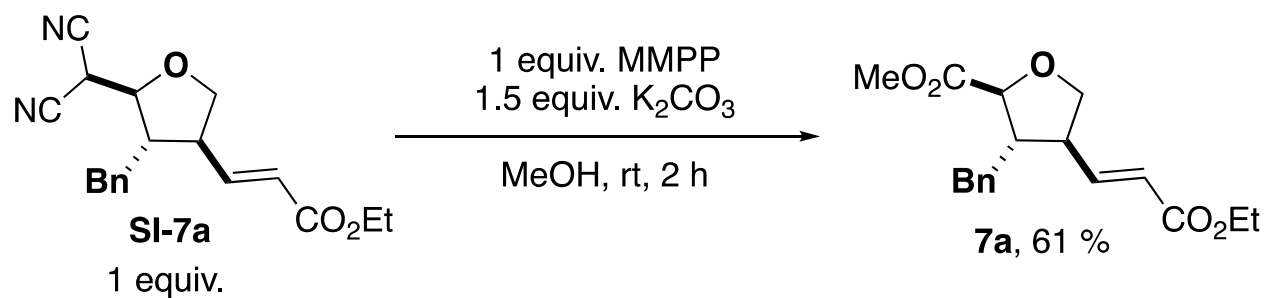

SI-7a (1 equiv., $10 \mathrm{mg}, 0.031 \mathrm{mmol})$ was dissolved in dry $\mathrm{MeOH}(1 \mathrm{~mL})$ in a flame-dried Schlenk flask under $\mathrm{N}_{2} . \mathrm{K}_{2} \mathrm{CO}_{3}$ (1.5 equiv., $6 \mathrm{mg}, 0.046 \mathrm{mmol}$ ) and MMPP (magnesium monoperoxyphthalate $80 \%, 1$ equiv., $12 \mathrm{mg}, 0.031 \mathrm{mmol}$ ) were added sequentially in this order at room temperature. After $2 \mathrm{~h}$ at room 
temperature, $\mathrm{MeOH}$ is removed under reduced pressure. The residue is diluted with EtOAc and was filtered through a short pad of silica to remove MMPP solid byproducts. The filtrate was then concentrated down and 7a was obtained clean without further purification. ( $6 \mathrm{mg}, 61 \%$, colorless oil, 6:1 d.r., major reported below).

${ }^{1}$ H NMR (600 MHz, Chloroform- $d$ ) $\delta 7.28(\mathrm{~d}, J=7.6 \mathrm{~Hz}, 3 \mathrm{H}), 7.20(\mathrm{tt}, J=7.6,1.4 \mathrm{~Hz}, 1 \mathrm{H}), 7.16(\mathrm{~d}, J=$ $6.7 \mathrm{~Hz}, 2 \mathrm{H}), 6.65(\mathrm{dd}, J=15.6,9.0 \mathrm{~Hz}, 1 \mathrm{H}), 5.72(\mathrm{dd}, J=15.7,0.9 \mathrm{~Hz}, 1 \mathrm{H}), 4.24(\mathrm{~d}, J=7.5 \mathrm{~Hz}, 1 \mathrm{H}), 4.16$ (q, $J=7.2 \mathrm{~Hz}, 2 \mathrm{H}), 4.07$ (dd, $J=8.8,7.4 \mathrm{~Hz}, 1 \mathrm{H}), 3.82$ (t, $J=8.9 \mathrm{~Hz}, 1 \mathrm{H}), 3.65(\mathrm{~s}, 3 \mathrm{H}), 2.89$ (h, $J=7.0$ $\mathrm{Hz}, 2 \mathrm{H}), 2.81-2.74(\mathrm{~m}, 1 \mathrm{H}), 2.57(\mathrm{dq}, J=8.7,7.1 \mathrm{~Hz}, 1 \mathrm{H}), 1.27(\mathrm{t}, J=7.1 \mathrm{~Hz}, 3 \mathrm{H})$.

${ }^{13}$ C NMR $\left(151 \mathrm{MHz}, \mathrm{CDCl}_{3}\right) \delta 172.9,165.9,145.6,137.9,129.6,128.6,126.9,123.6,81.5,72.9,60.6$, 52.3, 51.2, 48.1, 37.6, 14.4.

HRMS (ESI - TOF) m/z: Calcd for $\mathrm{C}_{18} \mathrm{H}_{22} \mathrm{NaO}_{5}[\mathrm{M}+\mathrm{Na}]^{+} 341.1359$, found 341.1360.

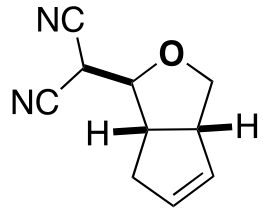

$8 \mathbf{a}$

1 equiv.

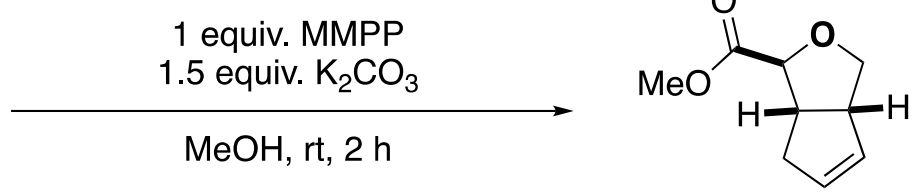

$8 b, 62 \%$

8a (1 equiv., $10 \mathrm{mg}, 0.057 \mathrm{mmol}$ ) was dissolved in dry $\mathrm{MeOH}(1 \mathrm{~mL})$ in a flame-dried Schlenk flask under $\mathrm{N}_{2} . \mathrm{K}_{2} \mathrm{CO}_{3}$ (1.5 equiv., $12 \mathrm{mg}, 0.086 \mathrm{mmol}$ ) and MMPP (magnesium monoperoxyphthalate $80 \%$, 1 equiv., $22 \mathrm{mg}, 0.057 \mathrm{mmol}$ ) were added sequentially in this order at room temperature. After $2 \mathrm{~h}$ at room temperature, $\mathrm{MeOH}$ is removed under reduced pressure. The residue is diluted with EtOAc and was filtered through a short pad of silica to remove MMPP solid byproducts. The filtrate was then concentrated down and $\mathbf{8 b}$ was obtained clean without further purification. (6 mg, $62 \%$, yellowish oil, single diastereomer).

${ }^{1}$ H NMR (600 MHz, Chloroform- $d$ ) $\delta 5.73$ (dq, $\left.J=4.6,2.2 \mathrm{~Hz}, 1 \mathrm{H}\right), 5.62-5.59(\mathrm{~m}, 1 \mathrm{H}), 4.18(\mathrm{dd}, J=8.7$, $7.1 \mathrm{~Hz}, 1 \mathrm{H}), 4.09(\mathrm{~d}, J=5.7 \mathrm{~Hz}, 1 \mathrm{H}), 3.79(\mathrm{dd}, J=8.8,3.1 \mathrm{~Hz}, 1 \mathrm{H}), 3.77(\mathrm{~s}, 3 \mathrm{H}), 3.46$ (dtd, $J=10.8,4.9$, $2.4 \mathrm{~Hz}, 1 \mathrm{H}), 2.98(\mathrm{tdd}, J=8.1,5.7,2.0 \mathrm{~Hz}, 1 \mathrm{H}), 2.71$ (ddq, $J=17.2,8.3,2.3 \mathrm{~Hz}, 1 \mathrm{H}), 2.45(\mathrm{dp}, J=17.3$, $2.2 \mathrm{~Hz}, 1 \mathrm{H})$.

${ }^{13}$ C NMR $\left(151 \mathrm{MHz}, \mathrm{CDCl}_{3}\right) \delta 173.4,132.0,130.6,84.7,73.6,52.2,51.9,46.4,38.6$.

HRMS (ESI - TOF) m/z: Calcd for $\mathrm{C}_{9} \mathrm{H}_{12} \mathrm{NaO}_{3}[\mathrm{M}+\mathrm{Na}]^{+}$191.0679, found 191.0671.

\section{vi. Retro-Oxy-Michael addition on 3a}

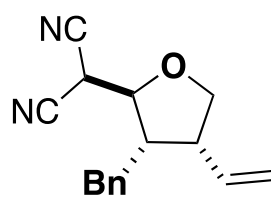

$3 a$

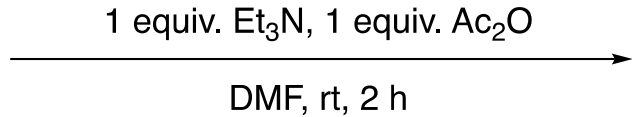

DMF, rt, 2 h

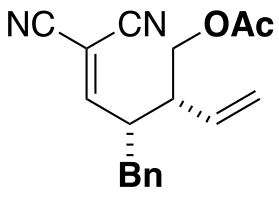

A, $60 \%$

3a (1 equiv., $10 \mathrm{mg}, 0.04 \mathrm{mmol})$ was dissolved in DMF $(1 \mathrm{~mL})$ at room temperature in a $4 \mathrm{~mL}$ vial under air. $\mathrm{Et}_{3} \mathrm{~N}$ (1.1 equiv., $6 \mu \mathrm{L}, 0.043 \mathrm{mmol}$ ) was added and the reaction was stirred for 5 min before $\mathrm{Ac}_{2} \mathrm{O}(1.1$ equiv., $4 \mu \mathrm{L}, 0.043 \mathrm{mmol}$ ) was added. The reaction mixture was stirred at room temeprature for $2 \mathrm{~h}$. After completion, the reaction mixture was diluted with $2 \mathrm{M} \mathrm{HCl}_{(\mathrm{aq})}(3 \mathrm{~mL})$ and extracted with EtOAc three times $(3 \times 1 \mathrm{~mL})$. The combined organic layers were washed with $2 \mathrm{M} \mathrm{HCl}_{(\mathrm{aq})}(3 \mathrm{~mL})$, brine $(6 \mathrm{~mL})$ and dried over $\mathrm{Na}_{2} \mathrm{SO}_{4}$. The solvent was removed under reduced pressure and the residue was purified via sílica gel column chromatography yielding compound A (7 mg, $60 \%)$. 
${ }^{1}$ H NMR (400 MHz, Chloroform- $d$ ) $\delta 7.39-7.29$ (m, 3H), $7.18-7.08$ (m, 3H), 5.72 (ddd, $J=17.0,10.4$, $9.0 \mathrm{~Hz}, 1 \mathrm{H}), 5.39(\mathrm{dd}, J=10.3,1.2 \mathrm{~Hz}, 1 \mathrm{H}), 5.29(\mathrm{dt}, J=17.1,1.0 \mathrm{~Hz}, 1 \mathrm{H}), 4.19(\mathrm{dd}, J=11.4,5.6 \mathrm{~Hz}, 1 \mathrm{H})$, 4.00 (dd, $J=11.4,7.7 \mathrm{~Hz}, 1 \mathrm{H}), 3.38$ (dddd, $J=11.2,9.1,5.8,4.7 \mathrm{~Hz}, 1 \mathrm{H}), 3.03$ (dd, $J=13.8,5.9 \mathrm{~Hz}, 1 \mathrm{H})$, $2.80-2.76(\mathrm{~m}, 1 \mathrm{H}), 2.72(\mathrm{dd}, J=13.8,9.2 \mathrm{~Hz}, 1 \mathrm{H}), 2.12(\mathrm{~s}, 3 \mathrm{H})$.

${ }^{13} \mathrm{C}$ NMR $\left(101 \mathrm{MHz}, \mathrm{CDCl}_{3}\right) \delta 170.8,169.3,136.6,132.7,129.1,129.0,127.5,121.2,111.8,110.3,91.3$, $77.2,64.8,46.3,46.1,38.3,21.0$.

\section{vii. Detailed representative large-scale reaction $(>1 \mathrm{mmol})$}

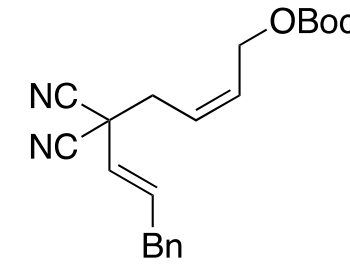

Z-1a, 1 equiv. $660 \mathrm{mg}(1.87 \mathrm{mmol})$

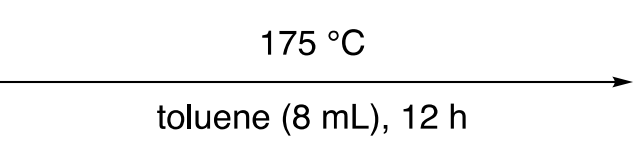

toluene $(8 \mathrm{~mL}), 12 \mathrm{~h}$<smiles>C=CC1COC(C(C#N)C#N)C1Br</smiles>

iso-3a

$395 \mathrm{mg}, 84 \%$

$8: 1$ d.r.

Z-1a (1 equiv., $660 \mathrm{mg}, 1.87 \mathrm{mmol}$ ) was dissolved in $8 \mathrm{~mL}$ toluene and charged in a Biotage ${ }^{\circledR}$ microwave reaction vial 10-20 mL. A small stir bar was added, and the vial was sealed with a cap with septum. The vial was placed in a Chemglass ${ }^{\circledR} 4$-place pie wedge for $10-20 \mathrm{~mL}$ microwave vials pre-heated at $175{ }^{\circ} \mathrm{C}$. The reaction mixture was then stirred at $175^{\circ} \mathrm{C}$ for exactly $12 \mathrm{~h}$ (controlled by a timer). After $12 \mathrm{~h}$, the reaction mixture was cooled down to room temperature. The solvent was then removed under reduced pressure and the residue was purified via silica gel column chromatography Hex/EtOAc $20 \%$ yielding iso3a (395 mg, 84 \% yield, 8:1 d.r., white solid). See iso-3a in the next section for analysis.

\section{3. ${ }^{1} \mathrm{H},{ }^{13} \mathrm{C}$ NMR \& mass spectrometry data}

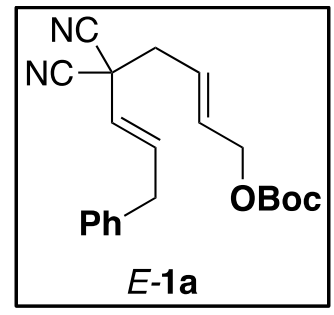

Synthesized according to Procedure B-1 (384 mg, $25 \%$ ).

Solvent system for column chromatography: Hex/EtOAc $10 \%$.

${ }^{1}$ H NMR (400 MHz, Chloroform- $d$ ): $\delta 7.33(\mathrm{tt}, J=7.8,1.6 \mathrm{~Hz}, 2 \mathrm{H}), 7.29-7.25(\mathrm{~m}, 1 \mathrm{H}), 7.15(\mathrm{~d}, J=7.0$ $\mathrm{Hz}, 2 \mathrm{H}), 6.38(\mathrm{dt}, J=15.2,6.6 \mathrm{~Hz}, 1 \mathrm{H}), 5.90(\mathrm{dtt}, J=15.4,5.7,0.8 \mathrm{~Hz}, 1 \mathrm{H}), 5.79(\mathrm{dtt}, J=15.4,7.2,1.3$ $\mathrm{Hz}, 1 \mathrm{H}), 5.38(\mathrm{dt}, J=15.1,1.6 \mathrm{~Hz}, 1 \mathrm{H}), 4.57(\mathrm{dd}, J=5.5,0.8 \mathrm{~Hz}, 2 \mathrm{H}), 3.48(\mathrm{dd}, J=6.4,1.6 \mathrm{~Hz}, 2 \mathrm{H}), 2.75$ (dd, $J=7.2,0.7 \mathrm{~Hz}, 2 \mathrm{H}), 1.50(\mathrm{~s}, 9 \mathrm{H})$.

${ }^{13}$ C NMR $\left(101 \mathrm{MHz}, \mathrm{CDCl}_{3}\right): \delta 153.2,137.6,137.5,133.0,129.0,128.8,127.0,124.1,121.6,114.0,82.7$, 66.0, 42.2, 38.9, 38.1, 27.9.

HRMS (ESI - TOF) m/z: Calcd for $\mathrm{C}_{21} \mathrm{H}_{24} \mathrm{~N}_{2} \mathrm{NaO}_{3}[\mathrm{M}+\mathrm{Na}]^{+} 375.1679$, found 375.1675. 


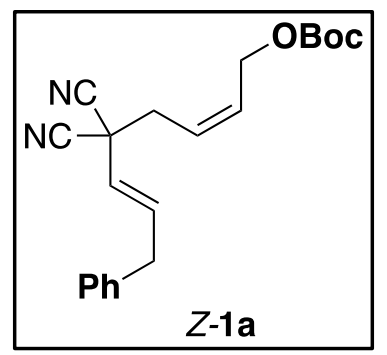

Synthesized according to Procedure A-1 (337 mg, $41 \%$ ).

Solvent system for column chromatography: Hex/EtOAc $10 \%$.

${ }^{1}$ H NMR (600 MHz, Chloroform- $d$ ) $\delta 7.36-7.31(\mathrm{~m}, 2 \mathrm{H}), 7.30-7.26(\mathrm{~m}, 1 \mathrm{H}), 7.19-7.14(\mathrm{~m}, 3 \mathrm{H}), 6.40$ $(\mathrm{dt}, J=15.2,6.6 \mathrm{~Hz}, 1 \mathrm{H}), 5.98(\mathrm{dtt}, J=11.2,6.9,1.4 \mathrm{~Hz}, 1 \mathrm{H}), 5.71(\mathrm{dtt}, J=10.8,7.7,1.4 \mathrm{~Hz}, 1 \mathrm{H}), 5.40$ $(\mathrm{dt}, J=15.2,1.7 \mathrm{~Hz}, 1 \mathrm{H}), 4.62(\mathrm{dd}, J=6.8,1.5 \mathrm{~Hz}, 2 \mathrm{H}), 3.48(\mathrm{dd}, J=6.5,1.7 \mathrm{~Hz}, 2 \mathrm{H}), 2.88(\mathrm{dd}, J=7.7$, $1.4 \mathrm{~Hz}, 2 \mathrm{H}), 1.48(\mathrm{~s}, 9 \mathrm{H})$.

${ }^{13}$ C NMR $\left(151 \mathrm{MHz}, \mathrm{CDCl}_{3}\right) \delta 153.3,137.6,131.3,129.0,128.8,127.0,124.3,121.5,114.0,82.8,61.9$, 38.7, 38.1, 37.4, 27.9.

HRMS (ESI - TOF) $\mathrm{m} / \mathrm{z}$ : Calcd for $\mathrm{C}_{21} \mathrm{H}_{24} \mathrm{~N}_{2} \mathrm{NaO}_{3}[\mathrm{M}+\mathrm{Na}]^{+} 375.1679$, found 375.1675.

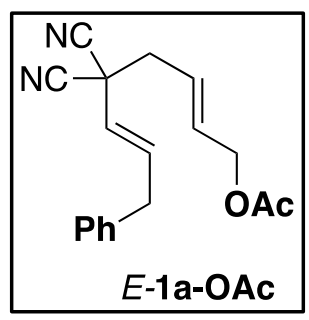

Synthesized according to Procedure B-1 with $\mathrm{Ac}_{2} \mathrm{O}$ instead of $\mathrm{Boc}_{2} \mathrm{O}(236 \mathrm{mg}, 49 \%)$.

Solvent system for column chromatography: Hex/EtOAc $10 \%$.

${ }^{1}$ H NMR (400 MHz, Chloroform- $d$ ) $\delta 7.37-7.30(\mathrm{~m}, 2 \mathrm{H}), 7.29-7.26(\mathrm{~m}, 1 \mathrm{H}), 7.18-7.13(\mathrm{~m}, 2 \mathrm{H}), 6.38$ $(\mathrm{dt}, J=15.2,6.6 \mathrm{~Hz}, 1 \mathrm{H}), 5.89(\mathrm{dtt}, J=15.3,5.6,1.1 \mathrm{~Hz}, 1 \mathrm{H}), 5.75(\mathrm{dtt}, J=15.6,7.3,1.4 \mathrm{~Hz}, 1 \mathrm{H}), 5.38$ $(\mathrm{dt}, J=15.2,1.7 \mathrm{~Hz}, 1 \mathrm{H}), 4.58(\mathrm{dd}, J=5.6,1.2 \mathrm{~Hz}, 2 \mathrm{H}), 3.49(\mathrm{dd}, J=6.6,1.6 \mathrm{~Hz}, 2 \mathrm{H}), 2.74(\mathrm{dd}, J=7.3$, $1.0 \mathrm{~Hz}, 2 \mathrm{H}), 2.08(\mathrm{~s}, 3 \mathrm{H})$.

${ }^{13}$ C NMR $\left(101 \mathrm{MHz}, \mathrm{CDCl}_{3}\right) \delta 170.7,137.6,137.5,133.2,129.0,128.8,127.0,123.8,121.6,114.0,63.7$, $42.2,39.0,38.1,21.0$.

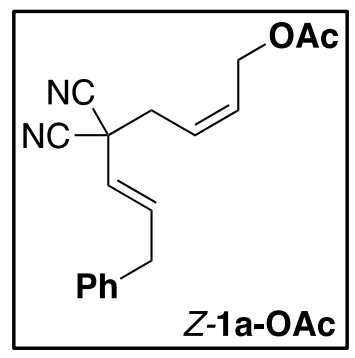

Synthesized according to Procedure A-1 with (Z)-4-bromobut-2-en-1-yl acetate (135 mg, 40 \%).

Solvent system for column chromatography: Hex/EtOAc $10 \%$.

${ }^{1}$ H NMR (400 MHz, Chloroform- $d$ ) $\delta 7.36$ - $7.31(\mathrm{~m}, 2 \mathrm{H}), 7.29-7.26(\mathrm{~m}, 1 \mathrm{H}), 7.23-7.09$ (m, 2H), 6.40 $(\mathrm{dt}, J=15.2,6.6 \mathrm{~Hz}, 1 \mathrm{H}), 5.96(\mathrm{dtt}, J=11.0,6.9,1.4 \mathrm{~Hz}, 1 \mathrm{H}), 5.70(\mathrm{dtt}, J=10.7,7.8,1.4 \mathrm{~Hz}, 1 \mathrm{H}), 5.40$ $(\mathrm{dt}, J=15.2,1.7 \mathrm{~Hz}, 1 \mathrm{H}), 4.63(\mathrm{dd}, J=7.0,1.4 \mathrm{~Hz}, 2 \mathrm{H}), 3.49$ (dd, $J=6.6,1.6 \mathrm{~Hz}, 2 \mathrm{H}), 2.87$ (dd, $J=7.6$, $1.4 \mathrm{~Hz}, 2 \mathrm{H}), 2.06(\mathrm{~s}, 3 \mathrm{H})$. 
${ }^{13}$ C NMR $\left(101 \mathrm{MHz}, \mathrm{CDCl}_{3}\right) \delta 170.8,137.5,137.5,131.4,129.0,128.8,127.0,124.2,121.5,114.0,59.6$, $38.8,38.1,37.4,21.0$.

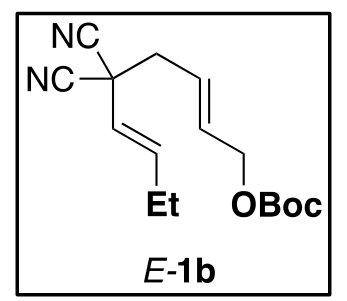

Synthesized according to Procedure B-1 (90 mg, $25 \%$ ).

Solvent system for column chromatography: Hex/EtOAc $10 \%$.

${ }^{1}$ H NMR (400 MHz, Chloroform- $d$ ) $\delta 6.24$ (dt, $\left.J=15.3,6.4 \mathrm{~Hz}, 1 \mathrm{H}\right), 5.92(\mathrm{dtt}, J=15.4,5.5,1.0 \mathrm{~Hz}, 1 \mathrm{H})$, $5.80(\mathrm{dtt}, J=15.5,7.3,1.3 \mathrm{~Hz}, 1 \mathrm{H}), 5.36(\mathrm{dt}, J=15.3,1.7 \mathrm{~Hz}, 1 \mathrm{H}), 4.59(\mathrm{dq}, J=5.7,0.9 \mathrm{~Hz}, 2 \mathrm{H}), 2.74$ (dd, $J=7.2,1.0 \mathrm{~Hz}, 2 \mathrm{H}), 2.23-2.14(\mathrm{~m}, 2 \mathrm{H}), 1.49(\mathrm{~s}, 9 \mathrm{H}), 1.06(\mathrm{t}, J=7.4 \mathrm{~Hz}, 3 \mathrm{H})$.

${ }^{13}$ C NMR $\left(101 \mathrm{MHz}, \mathrm{CDCl}_{3}\right) \delta 153.2,140.2,132.8,124.3,119.5,114.2,82.6,66.1,42.3,39.1,27.9,25.1$, 12.8 .

HRMS (ESI - TOF) m/z: Calcd for $\mathrm{C}_{16} \mathrm{H}_{22} \mathrm{~N}_{2} \mathrm{NaO}_{3}[\mathrm{M}+\mathrm{Na}]^{+}$313.1523, found 313.1522.

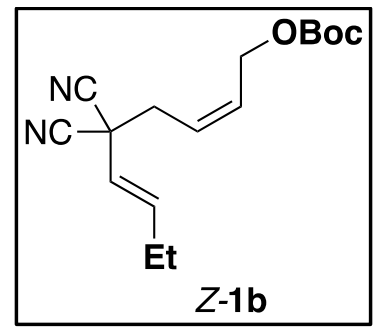

Synthesized according to Procedure A-1 (54 mg, $31 \%$ ).

Solvent system for column chromatography: Hex/EtOAc $10 \%$.

${ }^{1}$ H NMR $(400 \mathrm{MHz}$, Chloroform- $d$ ) $\delta 6.25(\mathrm{dt}, J=15.3,6.3 \mathrm{~Hz}, 1 \mathrm{H}), 5.98(\mathrm{dtt}, J=11.2,6.9,1.4 \mathrm{~Hz}, 1 \mathrm{H})$, $5.72(\mathrm{dtt}, J=10.7,7.7,1.5 \mathrm{~Hz}, 1 \mathrm{H}), 5.38$ (dt, $J=15.3,1.7 \mathrm{~Hz}, 1 \mathrm{H}), 4.63(\mathrm{dd}, J=7.0,1.4 \mathrm{~Hz}, 2 \mathrm{H}), 2.87$ (dd, $J=7.7,1.4 \mathrm{~Hz}, 2 \mathrm{H}), 2.18$ (qdd, $J=7.5,6.3,1.7 \mathrm{~Hz}, 2 \mathrm{H}), 1.48(\mathrm{~s}, 9 \mathrm{H}), 1.05(\mathrm{t}, J=7.4 \mathrm{~Hz}, 3 \mathrm{H})$.

${ }^{13} \mathbf{C}$ NMR $\left(101 \mathrm{MHz} \mathrm{CDCl}_{3}\right) \delta 153.3,140.2,131.1,124.4,119.5,114.2,82.7,61.9,38.7,37.4,27.9,25.1$, 12.7 .

HRMS (ESI - TOF) m/z: Calcd for $\mathrm{C}_{16} \mathrm{H}_{22} \mathrm{~N}_{2} \mathrm{NaO}_{3}[\mathrm{M}+\mathrm{Na}]^{+}$313.1523, found 313.1522.

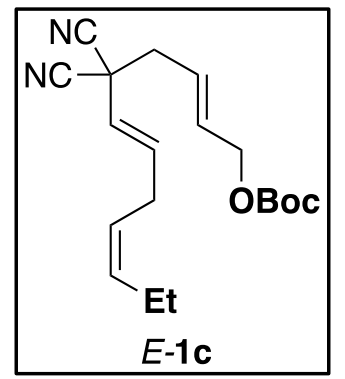

Synthesized according to Procedure B-1 (100 mg, $49 \%$ ).

Solvent system for column chromatography: Hex/EtOAc $10 \%$.

${ }^{1}$ H NMR (400 MHz, Chloroform- $d$ ) $\delta 6.20(\mathrm{dt}, J=15.3,6.0 \mathrm{~Hz}, 1 \mathrm{H}), 5.91(\mathrm{dtt}, J=15.4,5.6,0.5 \mathrm{~Hz}, 1 \mathrm{H})$, $5.84-5.74(\mathrm{~m}, 1 \mathrm{H}), 5.56(\mathrm{dtt}, J=10.6,7.4,1.2 \mathrm{~Hz}, 1 \mathrm{H}), 5.39(\mathrm{dt}, J=15.2,1.8 \mathrm{~Hz}, 1 \mathrm{H}), 5.31$ (dtt, $J=10.7$, 
$7.3,1.7 \mathrm{~Hz}, 1 \mathrm{H}), 4.58(\mathrm{~d}, J=5.6 \mathrm{~Hz}, 2 \mathrm{H}), 2.89(\mathrm{t}, J=6.6 \mathrm{~Hz}, 2 \mathrm{H}), 2.73(\mathrm{~d}, J=7.1 \mathrm{~Hz}, 2 \mathrm{H}), 2.11-1.96(\mathrm{~m}$, $2 \mathrm{H}), 1.49(\mathrm{~s}, 9 \mathrm{H}), 0.98(\mathrm{t}, J=7.5 \mathrm{~Hz}, 3 \mathrm{H})$.

${ }^{13}$ C NMR $\left(101 \mathrm{MHz}, \mathrm{CDCl}_{3}\right) \delta 153.2,137.0,135.0,132.9,124.2,123.5,120.5,114.1,82.6,66.1,42.2$, 39.1, 29.5, 27.9, 20.7, 14.3.

HRMS (ESI - TOF) m/z: Calcd for $\mathrm{C}_{19} \mathrm{H}_{26} \mathrm{~N}_{2} \mathrm{NaO}_{3}[\mathrm{M}+\mathrm{Na}]^{+}$353.1836, fouond 353.1827.

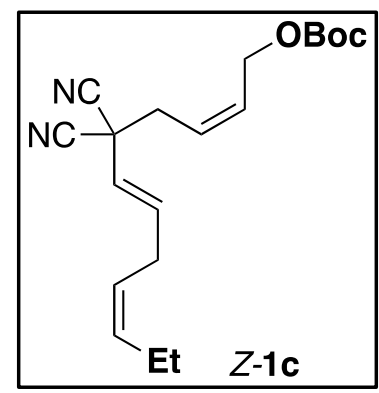

Synthesized according to Procedure A-2 (51 mg, $12 \%)$.

Solvent system for column chromatography: Hex/EtOAc $10 \%$.

${ }^{1}$ H NMR (400 MHz, Chloroform- $d$ ) $\delta 6.22(\mathrm{dt}, J=15.2,6.1 \mathrm{~Hz}, 1 \mathrm{H}), 5.98(\mathrm{dtt}, J=11.0,6.8,1.4 \mathrm{~Hz}, 1 \mathrm{H})$, $5.71(\mathrm{dtt}, J=10.7,7.7,1.5 \mathrm{~Hz}, 1 \mathrm{H}), 5.56(\mathrm{dtt}, J=10.3,7.3,1.5 \mathrm{~Hz}, 1 \mathrm{H}), 5.41(\mathrm{dt}, J=15.3,1.8 \mathrm{~Hz}, 1 \mathrm{H})$, $5.31(\mathrm{dtt}, J=10.6,7.3,1.6 \mathrm{~Hz}, 1 \mathrm{H}), 4.63(\mathrm{dd}, J=6.9,1.4 \mathrm{~Hz}, 2 \mathrm{H}), 2.94-2.84(\mathrm{~m}, 4 \mathrm{H}), 2.03(\mathrm{pd}, J=7.5$, $1.6 \mathrm{~Hz}, 3 \mathrm{H}), 1.48(\mathrm{~s}, 9 \mathrm{H}), 0.97(\mathrm{t}, J=7.5 \mathrm{~Hz}, 3 \mathrm{H})$.

${ }^{13}$ C NMR $\left(101 \mathrm{MHz}, \mathrm{CDCl}_{3}\right) \delta 153.3,137.1,135.0,131.2,124.3,123.5,120.5,114.1,82.7,61.9,38.7$, 37.4, 29.4, 27.9, 20.7, 14.2 .

HRMS (ESI - TOF) m/z: Calcd for $\mathrm{C}_{19} \mathrm{H}_{26} \mathrm{~N}_{2} \mathrm{NaO}_{3}[\mathrm{M}+\mathrm{Na}]^{+}$353.1836, fouond 353.1827.

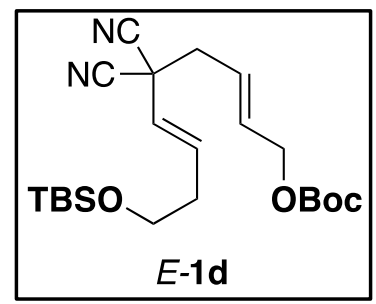

Synthesized according to Procedure B-1 (122 mg, $58 \%$ ).

Solvent system for column chromatography: Hex/EtOAc $5 \% \rightarrow 10 \%$.

${ }^{1}$ H NMR (400 MHz, Chloroform- $d$ ) $\delta 6.23$ (dt, $\left.J=15.3,7.0 \mathrm{~Hz}, 1 \mathrm{H}\right), 5.93$ (dtt, $\left.J=15.4,5.5,1.0 \mathrm{~Hz}, 1 \mathrm{H}\right)$, $5.80(\mathrm{dtt}, J=15.4,7.2,1.0 \mathrm{~Hz}, 1 \mathrm{H}), 5.46(\mathrm{dt}, J=15.3,1.5 \mathrm{~Hz}, 1 \mathrm{H}), 4.59(\mathrm{dd}, J=5.5,1.2 \mathrm{~Hz}, 2 \mathrm{H}), 3.70(\mathrm{t}$, $J=6.2 \mathrm{~Hz}, 2 \mathrm{H}), 2.74(\mathrm{~d}, J=7.6 \mathrm{~Hz}, 2 \mathrm{H}), 2.36(\mathrm{dtd}, J=7.6,6.2,1.5 \mathrm{~Hz}, 2 \mathrm{H}), 1.49$ (s, 9H), 0.89 (s, 9H), $0.06(\mathrm{~s}, 6 \mathrm{H})$.

${ }^{13}$ C NMR $\left(101 \mathrm{MHz}, \mathrm{CDCl}_{3}\right) \delta 153.2,135.7,132.9,128.1,124.3,122.0,114.0,82.6,66.1,62.4,61.6,42.3$, $39.2,35.4,27.9,26.0,18.4,-5.2$.

HRMS (ESI - TOF) m/z: Calcd for $\mathrm{C}_{22} \mathrm{H}_{36} \mathrm{~N}_{2} \mathrm{NaO}_{4} \mathrm{Si}[\mathrm{M}+\mathrm{Na}]^{+}$443.2337, found 443.2335.

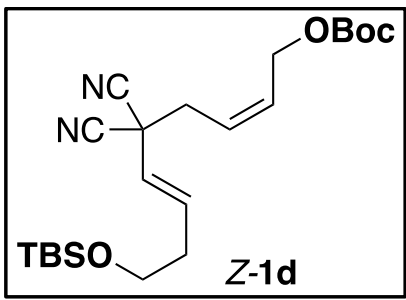


Synthesized according to Procedure A-2 (60 mg, $30 \% *)$.

*Note: $Z$-1d was isolated along with $10 \% \mathrm{SM}$, inseparable.

Solvent system for column chromatography: Hex/EtOAc $5 \% \rightarrow 10 \%$.

${ }^{1}$ H NMR $(400 \mathrm{MHz}$, Chloroform- $d$ ) $\delta 6.24(\mathrm{dt}, J=15.3,7.0 \mathrm{~Hz}, 1 \mathrm{H}), 5.98(\mathrm{dtt}, J=11.2,6.9,1.4 \mathrm{~Hz}, 1 \mathrm{H})$, $5.73(\mathrm{dtt}, J=11.1,7.5,1.4 \mathrm{~Hz}, 1 \mathrm{H}), 5.48(\mathrm{dt}, J=15.3,1.5 \mathrm{~Hz}, 1 \mathrm{H}), 4.64(\mathrm{dd}, J=6.9,1.4 \mathrm{~Hz}, 2 \mathrm{H}), 3.70(\mathrm{t}$, $J=6.2 \mathrm{~Hz}, 2 \mathrm{H}), 2.88(\mathrm{dd}, J=7.7,1.4 \mathrm{~Hz}, 2 \mathrm{H}), 2.41-2.31(\mathrm{~m}, 2 \mathrm{H}), 1.48$ (s, 9H), 0.89 (s, 9H), 0.06 (s, 6H). ${ }^{13}$ C NMR $\left(101 \mathrm{MHz}, \mathrm{CDCl}_{3}\right) \delta 153.3,135.8,131.2,124.4,121.9,114.1,82.7,61.9,61.6,38.9,37.4,35.4$, 27.9, 26.0, 18.4, -5.2.

HRMS (ESI - TOF) m/z: Calcd for $\mathrm{C}_{22} \mathrm{H}_{36} \mathrm{~N}_{2} \mathrm{NaO}_{4} \mathrm{Si}[\mathrm{M}+\mathrm{Na}]^{+}$443.2337, found 443.2335.

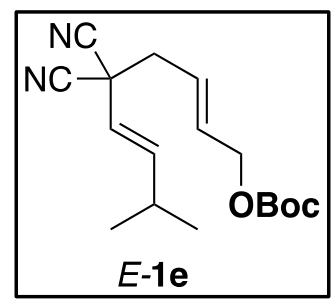

Synthesized according to Procedure B-1 (97 mg, $30 \%$ ).

Solvent system for column chromatography: Hex/EtOAc $10 \%$.

${ }^{1}$ H NMR $(400 \mathrm{MHz}$, Chloroform- $d$ ) $\delta 6.16(\mathrm{dd}, J=15.3,6.7 \mathrm{~Hz}, 1 \mathrm{H}), 5.92(\mathrm{dt}, J=15.5,5.5 \mathrm{~Hz}, 1 \mathrm{H}), 5.79$ $(\mathrm{dtt}, J=15.4,7.1,1.2 \mathrm{~Hz}, 1 \mathrm{H}), 5.32(\mathrm{dd}, J=15.3,1.4 \mathrm{~Hz}, 1 \mathrm{H}), 4.59(\mathrm{~d}, J=5.5 \mathrm{~Hz}, 2 \mathrm{H}), 2.74(\mathrm{~d}, J=7.3$ $\mathrm{Hz}, 2 \mathrm{H}), 2.47-2.37(\mathrm{~m}, 1 \mathrm{H}), 1.49(\mathrm{~s}, 9 \mathrm{H}), 1.05(\mathrm{~d}, J=6.7 \mathrm{~Hz}, 6 \mathrm{H})$.

${ }^{13} \mathbf{C}$ NMR $\left(101 \mathrm{MHz}, \mathrm{CDCl}_{3}\right) \delta 153.2,145.2,132.8,124.3,118.0,114.2,82.6,66.1,42.3,39.1,31.0,27.9$, 21.8 .

HRMS (ESI - TOF) m/z: Calcd for $\mathrm{C}_{17} \mathrm{H}_{24} \mathrm{~N}_{2} \mathrm{NaO}_{3}[\mathrm{M}+\mathrm{Na}]^{+} 327.1679$, found 327.1662.

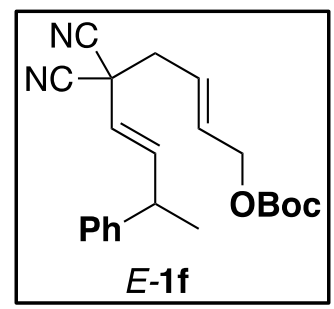

Synthesized according to Procedure B-1 (186 mg, $50 \%$ ).

Solvent system for column chromatography: Hex/EtOAc $10 \%$.

${ }^{1}$ H NMR (400 MHz, Chloroform- $d$ ) $\delta 7.34(\mathrm{t}, J=7.5 \mathrm{~Hz}, 2 \mathrm{H}), 7.29-7.26(\mathrm{~m}, 1 \mathrm{H}), 7.17$ (d, $J=7.3 \mathrm{~Hz}$, $2 \mathrm{H}), 6.37(\mathrm{dd}, J=15.3,6.5 \mathrm{~Hz}, 1 \mathrm{H}), 5.89(\mathrm{dt}, J=15.2,5.7 \mathrm{~Hz}, 1 \mathrm{H}), 5.77(\mathrm{dtt}, J=15.3,7.3,1.4 \mathrm{~Hz}, 1 \mathrm{H})$, $5.33(\mathrm{dd}, J=15.1,1.6 \mathrm{~Hz}, 1 \mathrm{H}), 4.56(\mathrm{~d}, J=5.2 \mathrm{~Hz}, 2 \mathrm{H}), 3.69-3.51(\mathrm{~m}, 1 \mathrm{H}), 2.74(\mathrm{~d}, J=7.2 \mathrm{~Hz}, 2 \mathrm{H}), 1.50$ $(\mathrm{s}, 9 \mathrm{H}), 1.42(\mathrm{~d}, J=7.0 \mathrm{~Hz}, 3 \mathrm{H})$.

${ }^{13}$ C NMR $\left(101 \mathrm{MHz}, \mathrm{CDCl}_{3}\right) \delta 153.2,143.2,143.0,133.0,129.0,127.3,127.1,124.1,119.7,114.1,114.0$, 82.6, 77.2, 66.0, 42.3, 41.9, 39.0, 27.9, 20.8.

HRMS (ESI - TOF) m/z: Calcd for $\mathrm{C}_{22} \mathrm{H}_{26} \mathrm{~N}_{2} \mathrm{NaO}_{3}[\mathrm{M}+\mathrm{Na}]^{+}$389.1836, found 389.1842. 


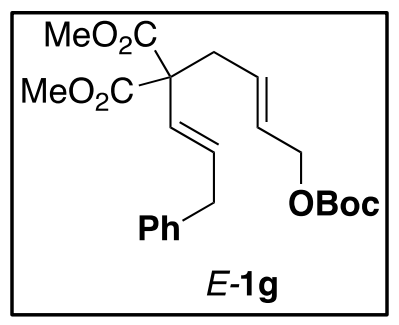

Synthesized according to Procedure B-2 (73 mg, $43 \%$ ).

Solvent system for column chromatography: Hex/EtOAc $10 \%$.

${ }^{1}$ H NMR (400 MHz, Chloroform- $d$ ) $\delta 7.32-7.27$ (m, 2H), 7.19 (dd, $\left.J=7.3,1.2 \mathrm{~Hz}, 1 \mathrm{H}\right), 7.16-7.12(\mathrm{~m}$, 2H), 5.99 (dt, $J=16.0,1.5 \mathrm{~Hz}, 1 \mathrm{H}), 5.71(\mathrm{dt}, J=16.0,6.9 \mathrm{~Hz}, 1 \mathrm{H}), 5.66-5.61(\mathrm{~m}, 2 \mathrm{H}), 4.44(\mathrm{~d}, J=4.7$ $\mathrm{Hz}, 2 \mathrm{H}), 3.73(\mathrm{~s}, 6 \mathrm{H}), 3.42(\mathrm{~d}, J=6.7 \mathrm{~Hz}, 2 \mathrm{H}), 2.80(\mathrm{~d}, J=5.5 \mathrm{~Hz}, 1 \mathrm{H}), 1.48(\mathrm{~s}, 9 \mathrm{H})$.

${ }^{13}$ C NMR $\left(101 \mathrm{MHz}, \mathrm{CDCl}_{3}\right) \delta 170.7,153.4,139.7,132.1,129.9,128.6,128.6,128.3,127.6,126.4,82.2$, $67.1,59.5,52.9,39.1,38.5,27.9$.

HRMS (ESI - TOF) m/z: Calcd for $\mathrm{C}_{23} \mathrm{H}_{30} \mathrm{NaO}_{7}[\mathrm{M}+\mathrm{Na}]^{+} 441.1884$, found 441.1888.

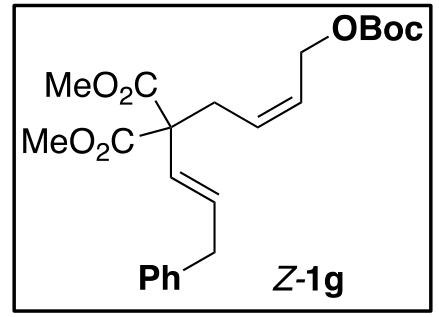

Synthesized according to Procedure A-2 (112 mg, $46 \%$ ).

Solvent system for column chromatography: Hex/EtOAc $10 \%$.

${ }^{1}$ H NMR (600 MHz, Chloroform- $\left.d\right) \delta 7.30-7.26(\mathrm{~m}, 2 \mathrm{H}), 7.19(\mathrm{tt}, J=7.5,1.2 \mathrm{~Hz}, 1 \mathrm{H}), 7.14(\mathrm{~d}, J=7.0$ $\mathrm{Hz}, 2 \mathrm{H}), 6.00(\mathrm{dt}, J=16.0,1.5 \mathrm{~Hz}, 1 \mathrm{H}), 5.74(\mathrm{dt}, J=16.0,6.9 \mathrm{~Hz}, 1 \mathrm{H}), 5.66(\mathrm{dtt}, J=11.1,6.8,1.5 \mathrm{~Hz}$, $1 \mathrm{H}), 5.52(\mathrm{dtt}, J=10.7,7.6,1.5 \mathrm{~Hz}, 1 \mathrm{H}), 4.57(\mathrm{dd}, J=6.7,1.5 \mathrm{~Hz}, 2 \mathrm{H}), 3.73(\mathrm{~s}, 6 \mathrm{H}), 3.43(\mathrm{~d}, J=6.7 \mathrm{~Hz}$, $2 \mathrm{H}), 2.86(\mathrm{dd}, J=7.5,1.6 \mathrm{~Hz}, 2 \mathrm{H}), 1.47(\mathrm{~s}, 9 \mathrm{H})$.

${ }^{13}$ C NMR $\left(151 \mathrm{MHz}, \mathrm{CDCl}_{3}\right) \delta 170.6,153.5,139.6,132.2,128.6,128.2,127.5,127.2,126.4,82.3,62.6$, 59.2, 52.9, 39.1, 33.6, 27.9.

HRMS (ESI - TOF) m/z: Calcd for $\mathrm{C}_{23} \mathrm{H}_{30} \mathrm{NaO}_{7}[\mathrm{M}+\mathrm{Na}]^{+} 441.1884$, found 441.1888 .

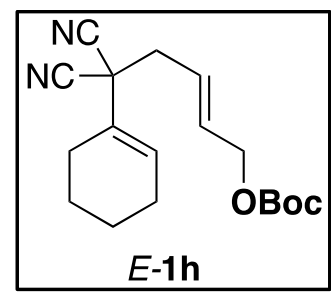

Synthesized according to Procedure B-1 (73 mg, $22 \%$ ).

Solvent system for column chromatography: Hex/EtOAc $10 \%$.

${ }^{1}$ H NMR (400 MHz, Chloroform- $d$ ) $\delta 6.21(\mathrm{tt}, J=3.8,1.5 \mathrm{~Hz}, 1 \mathrm{H}), 5.91(\mathrm{dtt}, J=15.3,5.6,1.0 \mathrm{~Hz}, 1 \mathrm{H})$, $5.76(\mathrm{dtt}, J=15.6,7.3,1.3 \mathrm{~Hz}, 1 \mathrm{H}), 4.58(\mathrm{dd}, J=5.7,1.2 \mathrm{~Hz}, 2 \mathrm{H}), 2.76(\mathrm{dd}, J=7.2,1.0 \mathrm{~Hz}, 2 \mathrm{H}), 2.22-$ $2.07(\mathrm{~m}, 4 \mathrm{H}), 1.77-1.69(\mathrm{~m}, 2 \mathrm{H}), 1.66-1.58(\mathrm{~m}, 2 \mathrm{H}), 1.49(\mathrm{~s}, 9 \mathrm{H})$.

${ }^{13} \mathrm{C}$ NMR $\left(101 \mathrm{MHz}, \mathrm{CDCl}_{3}\right) \delta 153.2,132.3,130.2,127.5,124.8,114.3,82.6,66.2,43.7,40.1,27.9,25.4$, 24.7, 22.4, 21.4.

HRMS (ESI - TOF) m/z: Calcd for $\mathrm{C}_{18} \mathrm{H}_{24} \mathrm{~N}_{2} \mathrm{NaO}_{3}[\mathrm{M}+\mathrm{Na}]^{+} 339.1679$, found 339.1674. 


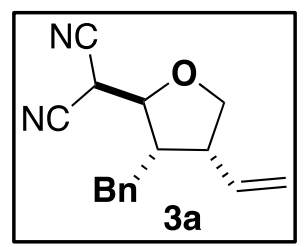

Synthesized according to Procedure C-1 (48 mg, $67 \%$, 5:1 d.r.). Solvent system for column chromatography: Hex/EtOAc $20 \%$.

${ }^{1}$ H NMR (400 MHz, Chloroform- $d$ ) $\delta 7.38-7.33(\mathrm{~m}, 2 \mathrm{H}), 7.32-7.27(\mathrm{~m}, 1 \mathrm{H}), 7.19(\mathrm{dd}, J=6.8,1.6 \mathrm{~Hz}$, 2H), 5.84 (ddd, $J=17.0,10.4,9.1 \mathrm{~Hz}, 1 \mathrm{H}), 5.29-5.19(\mathrm{~m}, 2 \mathrm{H}), 4.20(\mathrm{dd}, J=8.8,5.5 \mathrm{~Hz}, 1 \mathrm{H}), 4.06(\mathrm{dd}, J$ $=7.7,3.0 \mathrm{~Hz}, 1 \mathrm{H}), 3.90(\mathrm{dd}, J=8.8,3.6 \mathrm{~Hz}, 1 \mathrm{H}), 3.12(\mathrm{dtd}, J=9.3,6.0,3.7 \mathrm{~Hz}, 1 \mathrm{H}), 2.99(\mathrm{dd}, J=13.6$, $5.4 \mathrm{~Hz}, 1 \mathrm{H}), 2.87(\mathrm{~d}, J=2.9 \mathrm{~Hz}, 1 \mathrm{H}), 2.72-2.65(\mathrm{~m}, 1 \mathrm{H}), 2.54$ (dd, $J=13.6,10.5 \mathrm{~Hz}, 1 \mathrm{H})$.

${ }^{13}$ C NMR $\left(101 \mathrm{MHz}, \mathrm{CDCl}_{3}\right) \delta 138.5,134.5,129.4,128.6,127.5,118.7,111.6,110.9,80.8,74.0,48.2$, 47.3, 34.9, 28.8 .

HRMS (ESI - TOF) m/z: Calcd for $\mathrm{C}_{16} \mathrm{H}_{15} \mathrm{~N}_{2} \mathrm{O}[\mathrm{M}-\mathrm{H}]^{-}$251.1190, found 251.1182.

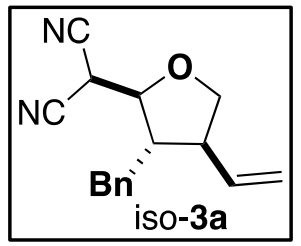

Synthesized according to Procedure C-1 (52 mg, 73 \%, 8:1 d.r.), see section 2.d.vii. for large-scale.

Solvent system for column chromatography: Hex/EtOAc $20 \%$.

${ }^{1}$ H NMR (400 MHz, Chloroform- $d$ ) $\delta 7.39-7.33(\mathrm{~m}, 2 \mathrm{H}), 7.33-7.27(\mathrm{~m}, 1 \mathrm{H}), 7.18(\mathrm{dd}, J=6.9,1.7 \mathrm{~Hz}$, $2 \mathrm{H}), 5.73(\mathrm{ddd}, J=17.0,10.2,8.5 \mathrm{~Hz}, 1 \mathrm{H}), 5.28-5.20(\mathrm{~m}, 2 \mathrm{H}), 4.12(\mathrm{dd}, J=8.7,7.7 \mathrm{~Hz}, 1 \mathrm{H}), 4.05(\mathrm{dd}, J$ $=8.6,2.5 \mathrm{~Hz}, 1 \mathrm{H}), 3.83(\mathrm{dd}, J=10.1,8.7 \mathrm{~Hz}, 1 \mathrm{H}), 3.17(\mathrm{dd}, J=13.7,4.5 \mathrm{~Hz}, 1 \mathrm{H}), 2.77(\mathrm{tt}, J=10.0,8.0$ $\mathrm{Hz}, 1 \mathrm{H}), 2.66(\mathrm{~d}, J=2.5 \mathrm{~Hz}, 1 \mathrm{H}), 2.49$ (dd, $J=13.7,10.3 \mathrm{~Hz}, 1 \mathrm{H}), 2.28$ (tdd, $J=10.2,8.5,4.4 \mathrm{~Hz}, 1 \mathrm{H})$.

${ }^{13}$ C NMR (101 MHz, $\left.\mathrm{CDCl}_{3}\right) \delta 138.1,134.8,129.6,128.6,127.7,119.3,111.6,111.0,82.0,73.4,51.1$, 50.4, 36.8, 28.7.

HRMS (ESI - TOF) m/z: Calcd for $\mathrm{C}_{16} \mathrm{H}_{15} \mathrm{~N}_{2} \mathrm{O}[\mathrm{M}-\mathrm{H}]^{-}$251.1190, found 251.1182.

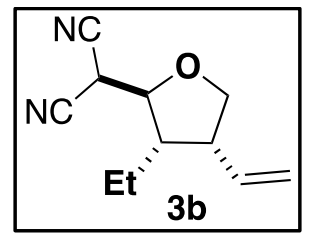

Synthesized according to Procedure C-1 (12 mg, $92 \%, 8: 1$ d.r.). Solvent system for column chromatography: Hex/EtOAc $20 \%$.

${ }^{1}$ H NMR (400 MHz, Chloroform- $d$ ) $\delta 5.74(\mathrm{ddd}, J=16.8,10.6,9.2 \mathrm{~Hz}, 1 \mathrm{H}), 5.21(\mathrm{dt}, J=2.3,1.0 \mathrm{~Hz}, 1 \mathrm{H})$, $5.20-5.16(\mathrm{~m}, 1 \mathrm{H}), 4.14(\mathrm{dd}, J=8.7,5.5 \mathrm{~Hz}, 1 \mathrm{H}), 4.07(\mathrm{dd}, J=7.3,4.0 \mathrm{~Hz}, 1 \mathrm{H}), 3.89(\mathrm{~d}, J=4.0 \mathrm{~Hz}, 1 \mathrm{H})$, $3.84(\mathrm{dd}, J=8.7,3.8 \mathrm{~Hz}, 1 \mathrm{H}), 3.07(\mathrm{dtd}, J=9.4,6.0,3.7 \mathrm{~Hz}, 1 \mathrm{H}), 2.26(\mathrm{p}, J=7.3 \mathrm{~Hz}, 1 \mathrm{H}), 1.56-1.45$ (m, $2 \mathrm{H}), 1.00(\mathrm{t}, J=7.3 \mathrm{~Hz}, 3 \mathrm{H})$.

${ }^{13}$ C NMR $\left(101 \mathrm{MHz}, \mathrm{CDCl}_{3}\right) \delta 134.1,118.3,111.6,111.0,80.7,74.1,48.9,46.3,28.8,21.1,12.2$.

HRMS (ESI - TOF) m/z: Calcd for $\mathrm{C}_{11} \mathrm{H}_{13} \mathrm{~N}_{2} \mathrm{O}[\mathrm{M}-\mathrm{H}]^{-}$189.1033, found 189.1023. 


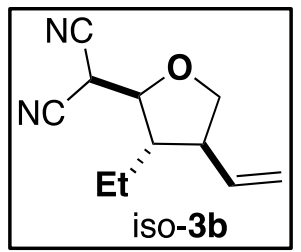

Synthesized according to Procedure C-1 (18 mg, $92 \%, 16: 1$ d.r.).

Solvent system for column chromatography: Hex/EtOAc $10 \%$.

${ }^{1}$ H NMR (400 MHz, Chloroform- $d$ ) $\delta 5.72(\mathrm{ddd}, J=17.1,10.2,8.5 \mathrm{~Hz}, 1 \mathrm{H}), 5.18$ (dt, $J=17.1,1.2 \mathrm{~Hz}$, 1H), 5.14 (dd, $J=10.2,1.4 \mathrm{~Hz}, 1 \mathrm{H}), 4.11-4.02(\mathrm{~m}, 2 \mathrm{H}), 3.89$ (d, $J=4.1 \mathrm{~Hz}, 1 \mathrm{H}), 3.80$ (t, $J=9.1 \mathrm{~Hz}, 1 \mathrm{H})$, $2.73(\mathrm{q}, J=8.1 \mathrm{~Hz}, 1 \mathrm{H}), 2.07-1.97(\mathrm{~m}, 1 \mathrm{H}), 1.64$ (qdd, $J=7.4,6.3,3.9 \mathrm{~Hz}, 2 \mathrm{H}), 1.01$ (t, $J=7.5 \mathrm{~Hz}, 3 \mathrm{H})$.

${ }^{13} \mathbf{C}$ NMR $\left(101 \mathrm{MHz}, \mathrm{CDCl}_{3}\right) \delta 136.2,118.2,111.6,111.1,81.8,73.7,50.6,50.5,28.9,24.2,11.8$.

HRMS (ESI - TOF) m/z: Calcd for $\mathrm{C}_{11} \mathrm{H}_{13} \mathrm{~N}_{2} \mathrm{O}[\mathrm{M}-\mathrm{H}]^{-}$189.1033, found 189.1023.

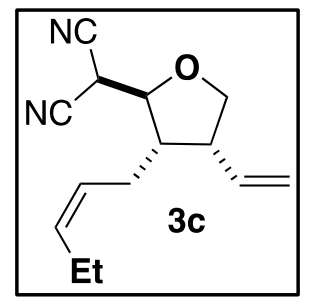

Synthesized according to Procedure C-1 (25 mg, $72 \%, 7: 1$ d.r.). Solvent system for column chromatography: Hex/EtOAc $10 \%$.

${ }^{1}$ H NMR (400 MHz, Chloroform- $d$ ) $\delta 5.76(\mathrm{ddd}, J=17.0,10.4,8.9 \mathrm{~Hz}, 1 \mathrm{H}), 5.54(\mathrm{dtt}, J=10.5,7.3,1.5$ $\mathrm{Hz}, 1 \mathrm{H}), 5.28(\mathrm{dtt}, J=10.8,6.5,1.7 \mathrm{~Hz}, 1 \mathrm{H}), 5.21(\mathrm{dd}, J=5.5,0.9 \mathrm{~Hz}, 1 \mathrm{H}), 5.18(\mathrm{dt}, J=14.1,1.1 \mathrm{~Hz}, 1 \mathrm{H})$, $4.17(\mathrm{dd}, J=8.7,5.8 \mathrm{~Hz}, 1 \mathrm{H}), 4.04(\mathrm{dd}, J=6.9,3.7 \mathrm{~Hz}, 1 \mathrm{H}), 3.92(\mathrm{~d}, J=3.7 \mathrm{~Hz}, 1 \mathrm{H}), 3.85(\mathrm{dd}, J=8.7,4.5$ $\mathrm{Hz}, 1 \mathrm{H}), 3.14-3.03(\mathrm{~m}, 1 \mathrm{H}), 2.39$ (dq, $J=8.3,7.1 \mathrm{~Hz}, 1 \mathrm{H}), 2.28-2.13(\mathrm{~m}, 2 \mathrm{H}), 2.10-1.99$ (m, 3H), 0.98 (t, $J=7.5 \mathrm{~Hz}, 3 \mathrm{H})$.

${ }^{13}$ C NMR $\left(101 \mathrm{MHz}, \mathrm{CDCl}_{3}\right) \delta 135.3,134.1,124.9,118.5,111.6,111.0,80.9,73.7,46.8,46.8,29.1,26.2$, 21.0, 14.2.

HRMS (ESI - TOF) m/z: Calcd for $\mathrm{C}_{14} \mathrm{H}_{17} \mathrm{~N}_{2} \mathrm{O}[\mathrm{M}-\mathrm{H}]^{-}$229.1335, found 229.1339.

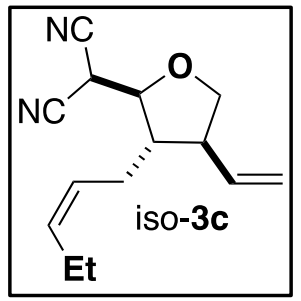

Synthesized according to Procedure C-1 (15 mg, $71 \%$, 10:1 d.r.).

Solvent system for column chromatography: Hex/EtOAc $10 \%$.

${ }^{1}$ H NMR (400 MHz, Chloroform- $d$ ) $\delta 5.69$ (ddd, $\left.J=17.0,10.2,8.5 \mathrm{~Hz}, 1 \mathrm{H}\right), 5.57$ (dtt, $J=11.0,7.3,1.5$ $\mathrm{Hz}, 1 \mathrm{H}), 5.31(\mathrm{dtt}, J=10.6,6.7,1.3 \mathrm{~Hz}, 1 \mathrm{H}), 5.23-5.14(\mathrm{~m}, 2 \mathrm{H}), 4.09(\mathrm{dd}, J=8.7,7.7 \mathrm{~Hz}, 1 \mathrm{H}), 4.03(\mathrm{dd}$, $J=8.2,3.2 \mathrm{~Hz}, 1 \mathrm{H}), 3.97(\mathrm{~d}, J=3.2 \mathrm{~Hz}, 1 \mathrm{H}), 3.81(\mathrm{dd}, J=9.8,8.7 \mathrm{~Hz}, 1 \mathrm{H}), 2.78-2.66(\mathrm{~m}, 1 \mathrm{H}), 2.43-$ $2.33(\mathrm{~m}, 1 \mathrm{H}), 2.24(\mathrm{dtd}, J=14.4,8.6,1.2 \mathrm{~Hz}, 1 \mathrm{H}), 2.10-2.02(\mathrm{~m}, 3 \mathrm{H}), 0.98(\mathrm{t}, J=7.5 \mathrm{~Hz}, 3 \mathrm{H})$.

${ }^{13} \mathbf{C}$ NMR $\left(101 \mathrm{MHz}, \mathrm{CDCl}_{3}\right) \delta 135.9,135.2,124.2,118.8,111.7,111.1,81.8,73.4,50.5,48.7,29.1,27.7$, 20.9, 14.2.

HRMS (ESI - TOF) m/z: Calcd for $\mathrm{C}_{14} \mathrm{H}_{17} \mathrm{~N}_{2} \mathrm{O}[\mathrm{M}-\mathrm{H}]^{-}$229.1335, found 229.1339. 


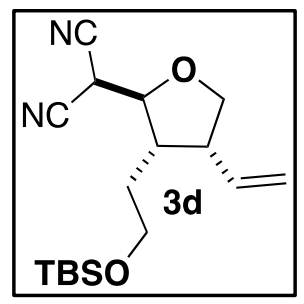

Synthesized according to Procedure C-1 (24 mg, $63 \%$, 14:1 d.r.).

Solvent system for column chromatography: Hex/EtOAc $10 \%$.

${ }^{1}$ H NMR (400 MHz, Chloroform- $d$ ) $\delta 5.74$ (ddd, $\left.J=17.0,10.4,9.4 \mathrm{~Hz}, 1 \mathrm{H}\right), 5.21-5.11(\mathrm{~m}, 2 \mathrm{H}), 4.44(\mathrm{~d}$, $J=2.7 \mathrm{~Hz}, 1 \mathrm{H}), 4.17(\mathrm{dd}, J=8.8,5.2 \mathrm{~Hz}, 1 \mathrm{H}), 4.13(\mathrm{dd}, J=8.2,2.7 \mathrm{~Hz}, 1 \mathrm{H}), 3.85(\mathrm{dd}, J=8.7,2.8 \mathrm{~Hz}$, $1 \mathrm{H}), 3.77(\mathrm{dt}, J=10.9,4.9 \mathrm{~Hz}, 1 \mathrm{H}), 3.64(\mathrm{ddd}, J=10.7,8.8,3.7 \mathrm{~Hz}, 1 \mathrm{H}), 3.08-2.93(\mathrm{~m}, 1 \mathrm{H}), 2.54-2.42$ $(\mathrm{m}, 1 \mathrm{H}), 1.76(\mathrm{dtd}, J=14.4,5.3,3.7 \mathrm{~Hz}, 1 \mathrm{H}), 1.55(\mathrm{ddt}, J=14.4,8.8,4.6 \mathrm{~Hz}, 1 \mathrm{H}), 0.90(\mathrm{~s}, 9 \mathrm{H}), 0.07(\mathrm{~s}$, $6 \mathrm{H})$.

${ }^{13}$ C NMR $\left(101 \mathrm{MHz}, \mathrm{CDCl}_{3}\right) \delta 135.0,118.2,112.2,111.5,81.3,73.9,62.1,47.9,45.2,31.8,28.5,26.1$, $18.4,-5.2,-5.3$.

HRMS (ESI - TOF) m/z: Calcd for $\mathrm{C}_{17} \mathrm{H}_{27} \mathrm{~N}_{2} \mathrm{O}_{2} \mathrm{Si}[\mathrm{M}-\mathrm{H}]^{-}$319.1836, found 319.1836.

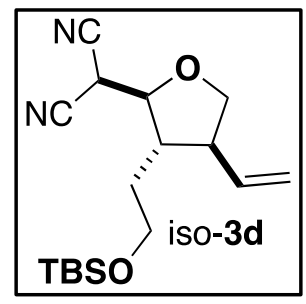

Synthesized according to Procedure C-1 ( $25 \mathrm{mg}, 66 \% *,>20: 1$ d.r.).

* Note: product iso-4c was isolated with $15 \%$ Knoevenagel adduct starting material, inseparable.

Solvent system for column chromatography: Hex/EtOAc $10 \%$.

${ }^{1}$ H NMR (400 MHz, Chloroform- $d$ ) $\delta 5.65(\mathrm{ddd}, J=17.0,10.1,8.6 \mathrm{~Hz}, 1 \mathrm{H}), 5.21-5.13(\mathrm{~m}, 2 \mathrm{H}), 4.65(\mathrm{~d}$, $J=2.2 \mathrm{~Hz}, 1 \mathrm{H}), 4.17(\mathrm{dd}, J=8.4,2.2 \mathrm{~Hz}, 1 \mathrm{H}), 4.08(\mathrm{dd}, J=8.6,7.7 \mathrm{~Hz}, 1 \mathrm{H}), 3.84-3.77(\mathrm{~m}, 2 \mathrm{H}), 3.62$ (ddd, $J=11.0,9.5,2.9 \mathrm{~Hz}, 1 \mathrm{H}), 2.67-2.58(\mathrm{~m}, 1 \mathrm{H}), 2.11-2.00(\mathrm{~m}, 1 \mathrm{H}), 1.90$ (ddt, $J=14.6,5.0,3.2 \mathrm{~Hz}$, $1 \mathrm{H}), 1.58-1.49(\mathrm{~m}, 1 \mathrm{H}), 0.90(\mathrm{~s}, 9 \mathrm{H}), 0.09(\mathrm{~s}, 6 \mathrm{H})$.

${ }^{13}$ C NMR $\left(101 \mathrm{MHz}, \mathrm{CDCl}_{3}\right) \delta 135.2,119.0,112.4,111.9,82.8,73.1,62.5,52.0,48.4,33.7,28.5,26.1$, $18.5,-5.2,-5.3$.

HRMS (ESI - TOF) m/z: Calcd for $\mathrm{C}_{17} \mathrm{H}_{27} \mathrm{~N}_{2} \mathrm{O}_{2} \mathrm{Si}[\mathrm{M}-\mathrm{H}]^{-}$319.1836, found 319.1836.

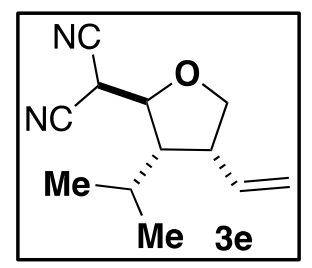

Synthesized according to Procedure C-1 (4.2 mg, $63 \%$ after 2 reaction cycle, 9:1 d.r.).

Solvent system for column chromatography: Hex/EtOAc $20 \%$.

${ }^{1}$ H NMR (400 MHz, Chloroform- $d$ ) $\delta 5.80(\mathrm{dt}, J=17.3,9.8 \mathrm{~Hz}, 1 \mathrm{H}), 5.22-5.18(\mathrm{~m}, 1 \mathrm{H}), 5.17(\mathrm{~s}, 1 \mathrm{H})$, $4.23(\mathrm{dd}, J=7.4,2.6 \mathrm{~Hz}, 1 \mathrm{H}), 4.19(\mathrm{dd}, J=8.6,5.3 \mathrm{~Hz}, 1 \mathrm{H}), 3.87(\mathrm{~d}, J=2.9 \mathrm{~Hz}, 1 \mathrm{H}), 3.85$ (d, $J=2.5 \mathrm{~Hz}$, $1 \mathrm{H}), 3.13-3.01(\mathrm{~m}, 1 \mathrm{H}), 2.10(\mathrm{dt}, J=9.0,7.1 \mathrm{~Hz}, 1 \mathrm{H}), 1.81(\mathrm{dp}, J=9.0,6.7 \mathrm{~Hz}, 1 \mathrm{H}), 1.00(\mathrm{~d}, J=6.6 \mathrm{~Hz}$, $3 \mathrm{H}), 0.95(\mathrm{~d}, J=6.7 \mathrm{~Hz}, 3 \mathrm{H})$.

${ }^{13}$ C NMR $\left(101 \mathrm{MHz}, \mathrm{CDCl}_{3}\right) \delta 134.7,118.0,111.9,111.4,79.6,74.7,53.8,47.4,30.3,27.3,21.9,21.3$. 
HRMS (ESI - TOF) m/z: Calcd for $\mathrm{C}_{12} \mathrm{H}_{15} \mathrm{~N}_{2} \mathrm{O}[\mathrm{M}-\mathrm{H}]^{-}$203.1179, found 203.1179.

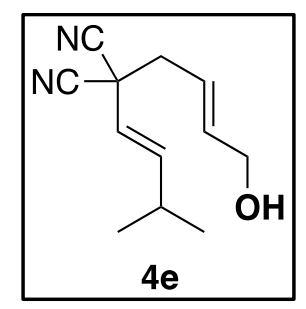

Deprotected by-product $E$-1e recovered after Procedure C-1 on $\mathbf{3 e}$.

${ }^{1}$ H NMR (400 MHz, Chloroform- $d$ ) $\delta 6.17(\mathrm{dd}, J=15.3,6.7 \mathrm{~Hz}, 1 \mathrm{H}), 5.99(\mathrm{dtt}, J=15.3,5.0,1.1 \mathrm{~Hz}, 1 \mathrm{H})$, $5.77(\mathrm{dtt}, J=15.1,7.3,1.7 \mathrm{~Hz}, 1 \mathrm{H}), 5.34(\mathrm{dd}, J=15.3,1.4 \mathrm{~Hz}, 1 \mathrm{H}), 4.22(\mathrm{ddt}, J=5.1,1.8,0.9 \mathrm{~Hz}, 2 \mathrm{H})$, $2.73(\mathrm{dq}, J=7.4,1.1 \mathrm{~Hz}, 2 \mathrm{H}), 2.43(\mathrm{dqd}, J=13.5,6.8,1.5 \mathrm{~Hz}, 1 \mathrm{H}), 1.06(\mathrm{~d}, J=6.8 \mathrm{~Hz}, 6 \mathrm{H})$.

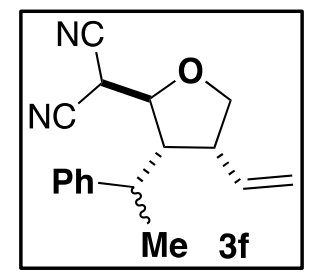

Synthesized according to Procedure C-1 (7.5 mg, 34 \%, 1:1 d.r., separable diastereomers).

Solvent system for column chromatography: Hex/EtOAc $20 \%$.

Diastereomer 1:

${ }^{1}$ H NMR (400 MHz, Chloroform- $d$ ) $\delta 7.42-7.36(\mathrm{~m}, 2 \mathrm{H}), 7.35-7.30(\mathrm{~m}, 1 \mathrm{H}), 7.22-7.18(\mathrm{~m}, 2 \mathrm{H}), 5.92$ $(\mathrm{dt}, J=17.4,9.9 \mathrm{~Hz}, 1 \mathrm{H}), 5.32(\mathrm{dd}, J=3.5,1.3 \mathrm{~Hz}, 1 \mathrm{H}), 5.29(\mathrm{~d}, J=1.2 \mathrm{~Hz}, 1 \mathrm{H}), 4.17(\mathrm{dd}, J=8.7,4.4 \mathrm{~Hz}$, $1 \mathrm{H}), 3.95(\mathrm{~d}, J=2.4 \mathrm{~Hz}, 1 \mathrm{H}), 3.93(\mathrm{~d}, J=1.9 \mathrm{~Hz}, 1 \mathrm{H}), 3.10(\mathrm{ddd}, J=10.1,6.0,4.2 \mathrm{~Hz}, 1 \mathrm{H}), 2.68(\mathrm{dq}, J=$ $11.2,6.7 \mathrm{~Hz}, 1 \mathrm{H}), 2.44(\mathrm{ddd}, J=11.3,9.4,6.1 \mathrm{~Hz}, 1 \mathrm{H}), 1.84(\mathrm{~d}, J=1.9 \mathrm{~Hz}, 1 \mathrm{H}), 1.37(\mathrm{~d}, J=6.7 \mathrm{~Hz}, 3 \mathrm{H})$. ${ }^{13}$ C NMR $\left(101 \mathrm{MHz}, \mathrm{CDCl}_{3}\right) \delta 144.0,134.6,129.8,128.2,126.9,118.4,111.9,111.2,80.1,74.6,53.9$, 47.1, 39.3, 28.5, 19.9.

HRMS (ESI - TOF) m/z: Calcd for $\mathrm{C}_{17} \mathrm{H}_{17} \mathrm{~N}_{2} \mathrm{O}[\mathrm{M}-\mathrm{H}]^{-}$265.1335, found 265.1339.

Diastereomer 2:

${ }^{1}$ H NMR (400 MHz, Chloroform- $d$ ) $\delta 7.36-7.29(\mathrm{~m}, 2 \mathrm{H}), 7.26-7.21(\mathrm{~m}, 1 \mathrm{H}), 7.20-7.16(\mathrm{~m}, 2 \mathrm{H}), 5.79$ (ddd, $J=17.2,10.4,8.9 \mathrm{~Hz}, 1 \mathrm{H}), 5.14(\mathrm{dd}, J=10.3,1.3 \mathrm{~Hz}, 1 \mathrm{H}), 4.88(\mathrm{dt}, J=17.2,1.2 \mathrm{~Hz}, 1 \mathrm{H}), 4.33$ (dd, $J=7.4,2.5 \mathrm{~Hz}, 1 \mathrm{H}), 4.19$ (dd, $J=8.7,5.2 \mathrm{~Hz}, 1 \mathrm{H}), 3.86(\mathrm{dd}, J=8.7,3.1 \mathrm{~Hz}, 1 \mathrm{H}), 3.80(\mathrm{~d}, J=2.5 \mathrm{~Hz}, 1 \mathrm{H})$, $2.96(\mathrm{dq}, J=9.7,7.0 \mathrm{~Hz}, 1 \mathrm{H}), 2.88-2.79(\mathrm{~m}, 1 \mathrm{H}), 2.72(\mathrm{dt}, J=9.4,7.1 \mathrm{~Hz}, 1 \mathrm{H}), 1.26(\mathrm{~d}, J=7.0 \mathrm{~Hz}, 3 \mathrm{H})$. ${ }^{13}$ C NMR $\left(101 \mathrm{MHz}, \mathrm{CDCl}_{3}\right) \delta 144.3,134.2,128.9,127.3,127.0,118.7,111.8,111.2,79.4,74.5,52.7$, 47.1, 38.7, 30.4, 21.1 .

HRMS (ESI - TOF) m/z: Calcd for $\mathrm{C}_{17} \mathrm{H}_{17} \mathrm{~N}_{2} \mathrm{O}[\mathrm{M}-\mathrm{H}]^{-}$265.1335, found 265.1339.

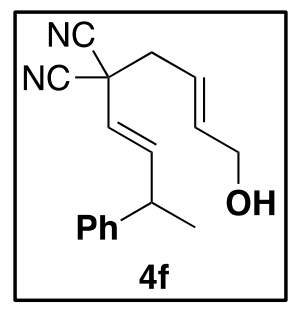

Deprotected by-product $E$-1f recovered after Procedure C-1 on 3f. (8 mg, 34 \%).

${ }^{1}$ H NMR (400 MHz, Chloroform- $d$ ) $\delta 7.38-7.31(\mathrm{~m}, 2 \mathrm{H}), 7.28-7.25(\mathrm{~m}, 1 \mathrm{H}), 7.20-7.15(\mathrm{~m}, 2 \mathrm{H}), 6.37$ $(\mathrm{dd}, J=15.3,6.6 \mathrm{~Hz}, 1 \mathrm{H}), 5.95(\mathrm{dtt}, J=15.4,5.0,1.2 \mathrm{~Hz}, 1 \mathrm{H}), 5.72(\mathrm{dtt}, J=15.1,7.4,1.7 \mathrm{~Hz}, 1 \mathrm{H}), 5.36$ 
$(\mathrm{dd}, J=15.3,1.5 \mathrm{~Hz}, 1 \mathrm{H}), 4.17(\mathrm{dd}, J=5.0,1.6 \mathrm{~Hz}, 2 \mathrm{H}), 3.59(\mathrm{pd}, J=7.0,1.5 \mathrm{~Hz}, 1 \mathrm{H}), 2.74(\mathrm{dt}, J=7.4$, $1.0 \mathrm{~Hz}, 2 \mathrm{H}), 1.43(\mathrm{~d}, J=7.1 \mathrm{~Hz}, 3 \mathrm{H})$.

${ }^{13}$ C NMR $\left(101 \mathrm{MHz}, \mathrm{CDCl}_{3}\right) \delta 143.28,142.79,138.34,128.95,127.30,127.07,120.78,119.78,114.23$, 114.17, 62.68, 42.29, 41.92, 39.31, 20.77.

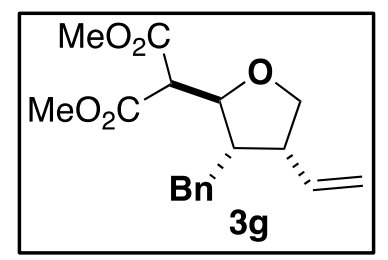

Synthesized according to Procedure C-2 (3 mg, $34 \%$ after 2 reaction cycle, 8:1 d.r.).

Solvent system for column chromatography: Hex/EtOAc $20 \%$.

${ }^{1}$ H NMR (600 MHz, Chloroform- $d$ ) $\delta 7.28(\mathrm{t}, J=7.6 \mathrm{~Hz}, 3 \mathrm{H}), 7.21-7.18(\mathrm{~m}, 1 \mathrm{H}), 7.16(\mathrm{~d}, J=6.6 \mathrm{~Hz}$, 2H), 5.87 (ddd, $J=17.1,10.3,8.8 \mathrm{~Hz}, 1 \mathrm{H}), 5.17$ (dd, $J=10.4,1.7 \mathrm{~Hz}, 1 \mathrm{H}), 5.09$ (dt, $J=17.1,1.2 \mathrm{~Hz}, 1 \mathrm{H}$ ), $4.39(\mathrm{dd}, J=7.2,5.8 \mathrm{~Hz}, 1 \mathrm{H}), 3.98(\mathrm{dd}, J=8.5,6.2 \mathrm{~Hz}, 1 \mathrm{H}), 3.79-3.76(\mathrm{~m}, 1 \mathrm{H}), 3.72(\mathrm{~s}, 3 \mathrm{H}), 3.56(\mathrm{~s}$, $3 \mathrm{H}), 3.36(\mathrm{~d}, J=7.2 \mathrm{~Hz}, 1 \mathrm{H}), 2.94(\mathrm{dq}, J=8.5,6.2 \mathrm{~Hz}, 1 \mathrm{H}), 2.79(\mathrm{dd}, J=13.7,7.0 \mathrm{~Hz}, 1 \mathrm{H}), 2.63-2.56$ $(\mathrm{m}, 1 \mathrm{H}), 2.50(\mathrm{dd}, J=13.7,8.2 \mathrm{~Hz}, 1 \mathrm{H})$.

${ }^{13}$ C NMR $\left(151 \mathrm{MHz}, \mathrm{CDCl}_{3}\right) \delta 167.7,167.5,139.9,135.4,129.1,128.6,126.4,117.9,80.7,72.3,56.7$, $52.7,52.7,48.0,46.5,34.4$.

HRMS (ESI - TOF) m/z: Calcd for $\mathrm{C}_{18} \mathrm{H}_{22} \mathrm{NaO}_{5}[\mathrm{M}+\mathrm{Na}]^{+} 341.1559$, found 341.1351 .

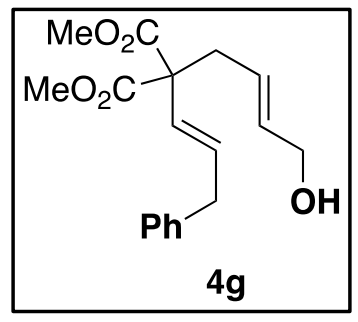

Deprotected by-product $E$-1g recovered after Procedure C-2 on $\mathbf{3 g}$ (1.8 mg, $21 \%$ after 2 reaction cycle).

${ }^{1}$ H NMR $(600 \mathrm{MHz}$, Chloroform- $d) \delta 7.29(\mathrm{t}, J=7.6 \mathrm{~Hz}, 2 \mathrm{H}), 7.22-7.18(\mathrm{~m}, 1 \mathrm{H}), 7.17-7.13(\mathrm{~m}, 2 \mathrm{H})$, $5.99(\mathrm{dt}, J=16.0,1.5 \mathrm{~Hz}, 1 \mathrm{H}), 5.73(\mathrm{dt}, J=16.0,7.0 \mathrm{~Hz}, 1 \mathrm{H}), 5.67(\mathrm{dtt}, J=15.4,5.7,1.3 \mathrm{~Hz}, 1 \mathrm{H}), 5.54$ $(\mathrm{dtt}, J=15.6,7.2,1.4 \mathrm{~Hz}, 1 \mathrm{H}), 4.02(\mathrm{dd}, J=5.7,1.4 \mathrm{~Hz}, 2 \mathrm{H}), 3.73(\mathrm{~s}, 6 \mathrm{H}), 3.43(\mathrm{~d}, J=7.0 \mathrm{~Hz}, 2 \mathrm{H}), 2.80$ (dd, $J=7.3,1.3 \mathrm{~Hz}, 2 \mathrm{H}$ ).

${ }^{13}$ C NMR $\left(151 \mathrm{MHz}, \mathrm{CDCl}_{3}\right) \delta 170.79,139.76,133.76,132.11,128.64,128.60,127.65,126.39,126.28$, $63.45,59.62,52.84,39.16,38.36$.

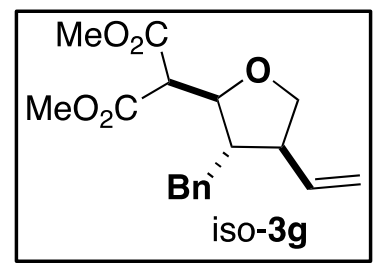

Synthesized according to Procedure C-2 (1.5 mg, $39 \%$ after 2 reaction cycle, $14: 1$ d.r.). Solvent system for column chromatography: Hex/EtOAc $20 \%$.

${ }^{1}$ H NMR (400 MHz, Chloroform- $d$ ) $\delta 7.30-7.26(\mathrm{~m}, 2 \mathrm{H}), 7.22-7.18(\mathrm{~m}, 1 \mathrm{H}), 7.17-7.12(\mathrm{~m}, 2 \mathrm{H}), 5.59$ (ddd, $J=17.8,9.9,8.2 \mathrm{~Hz}, 1 \mathrm{H}), 5.03-4.97(\mathrm{~m}, 1 \mathrm{H}), 4.96(\mathrm{t}, J=1.1 \mathrm{~Hz}, 1 \mathrm{H}), 4.36(\mathrm{t}, J=6.7 \mathrm{~Hz}, 1 \mathrm{H}), 3.95$ 
$(\mathrm{dd}, J=8.7,7.4 \mathrm{~Hz}, 1 \mathrm{H}), 3.73(\mathrm{~s}, 3 \mathrm{H}), 3.68(\mathrm{~s}, 3 \mathrm{H}), 3.61(\mathrm{t}, J=8.6 \mathrm{~Hz}, 1 \mathrm{H}), 3.26(\mathrm{~d}, J=6.4 \mathrm{~Hz}, 1 \mathrm{H}), 2.80$ (dd, $J=13.7,6.9 \mathrm{~Hz}, 1 \mathrm{H}), 2.72(\mathrm{dd}, J=13.8,7.1 \mathrm{~Hz}, 1 \mathrm{H}), 2.64(\mathrm{p}, J=8.1 \mathrm{~Hz}, 1 \mathrm{H}), 2.37-2.26(\mathrm{~m}, 1 \mathrm{H})$.

${ }^{13}$ C NMR $\left(101 \mathrm{MHz}, \mathrm{CDCl}_{3}\right) \delta 167.9,167.7,139.1,137.4,129.3,128.6,126.6,116.8,82.1,72.2,56.2$, 52.7, 52.6, 50.2, 50.1, 38.4.

HRMS (ESI - TOF) m/z: Calcd for $\mathrm{C}_{18} \mathrm{H}_{22} \mathrm{NaO}_{5}[\mathrm{M}+\mathrm{Na}]^{+} 341.1559$, found 341.1351.

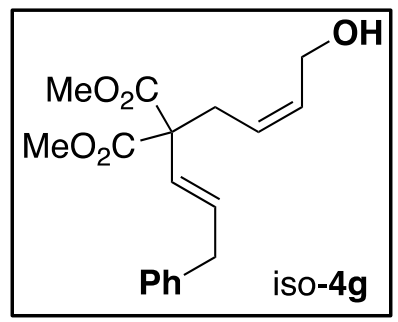

Deprotected by-product Z-1g recovered after Procedure C-2 on iso-3g $(\sim 1 \mathrm{mg}, 27 \%$ after 2 reaction cycle). ${ }^{1}$ H NMR (400 MHz, Chloroform- $d$ ) $\delta 7.35-7.29(\mathrm{~m}, 2 \mathrm{H}), 7.26-7.20(\mathrm{~m}, 1 \mathrm{H}), 7.20-7.15(\mathrm{~m}, 2 \mathrm{H}), 6.03$ $(\mathrm{dt}, J=16.0,1.5 \mathrm{~Hz}, 1 \mathrm{H}), 5.81-5.70(\mathrm{~m}, 2 \mathrm{H}), 5.44(\mathrm{dtt}, J=10.6,7.7,1.4 \mathrm{~Hz}, 1 \mathrm{H}), 4.15(\mathrm{~d}, J=6.8 \mathrm{~Hz}$, $2 \mathrm{H}), 3.76(\mathrm{~s}, 6 \mathrm{H}), 3.45(\mathrm{~d}, J=6.9 \mathrm{~Hz}, 2 \mathrm{H}), 2.86(\mathrm{dd}, J=7.7,1.5 \mathrm{~Hz}, 2 \mathrm{H})$.

\section{Structural and Stereochemical assignment control experiments}

a. Conversion and diastereoselectivity ratio for Scheme 4

Below are crude NMRs for 3a, iso-3a and their respective Cope products. An overlay of the starting material (in purple) is presented against the crude mixture (in green). All peaks from each diastereomers (pink and blue) and starting material could be clearly assigned. Each red box demonstrate how conversion and diastereoselective was determined. 

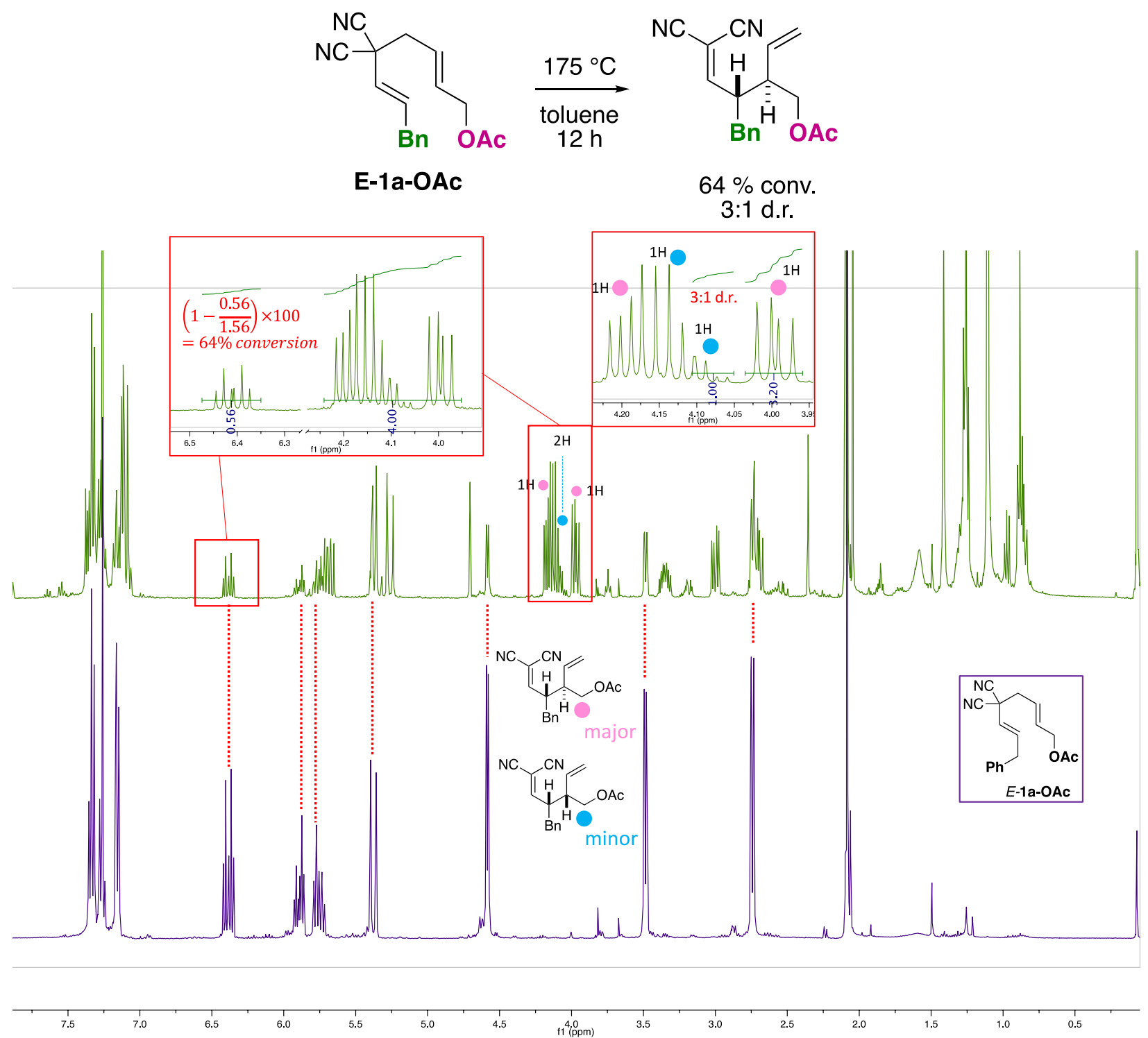


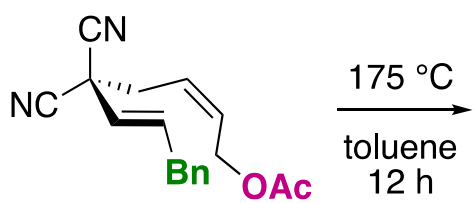

Z-1a-OAc

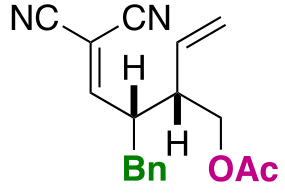

$74 \%$ conv.
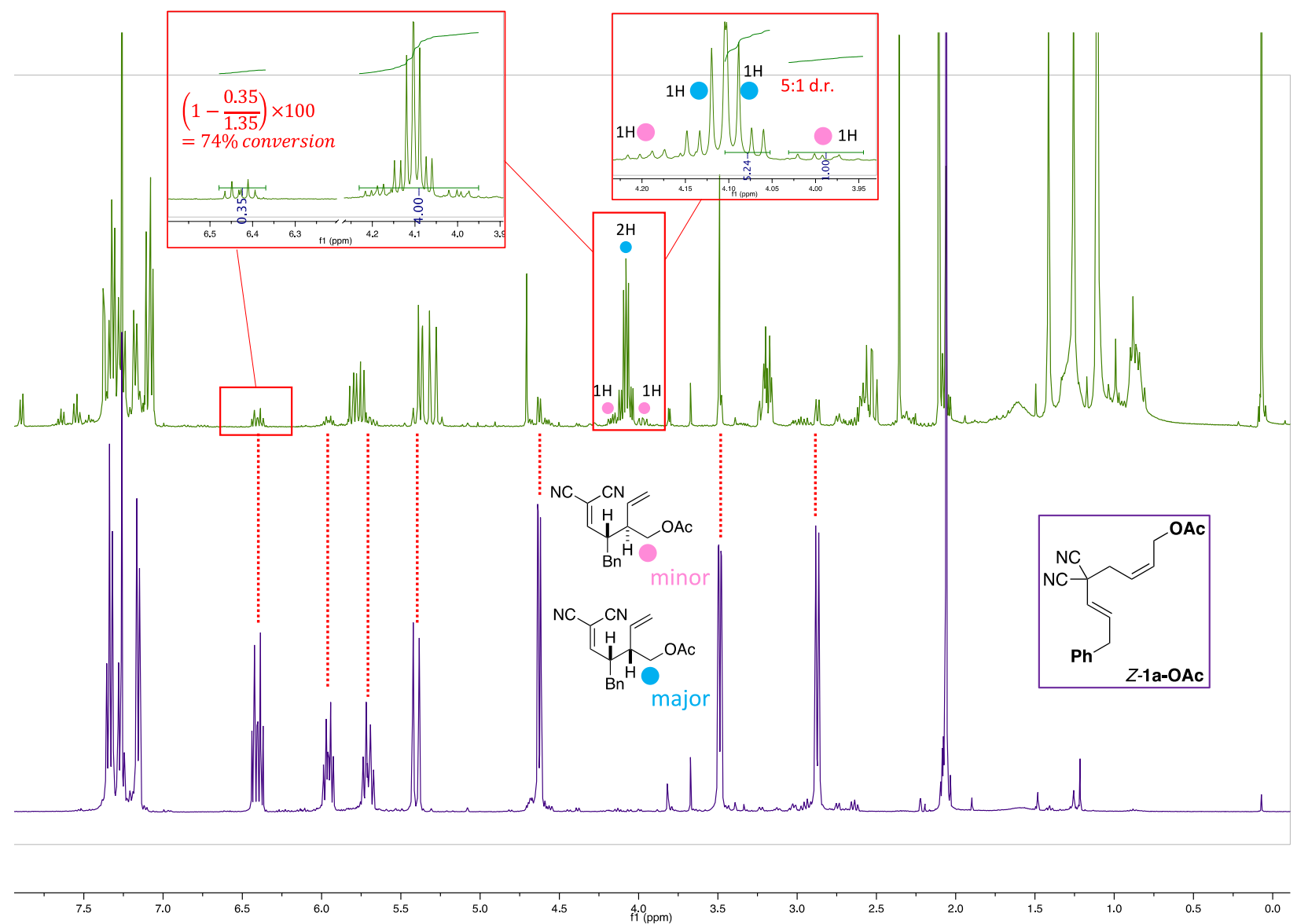

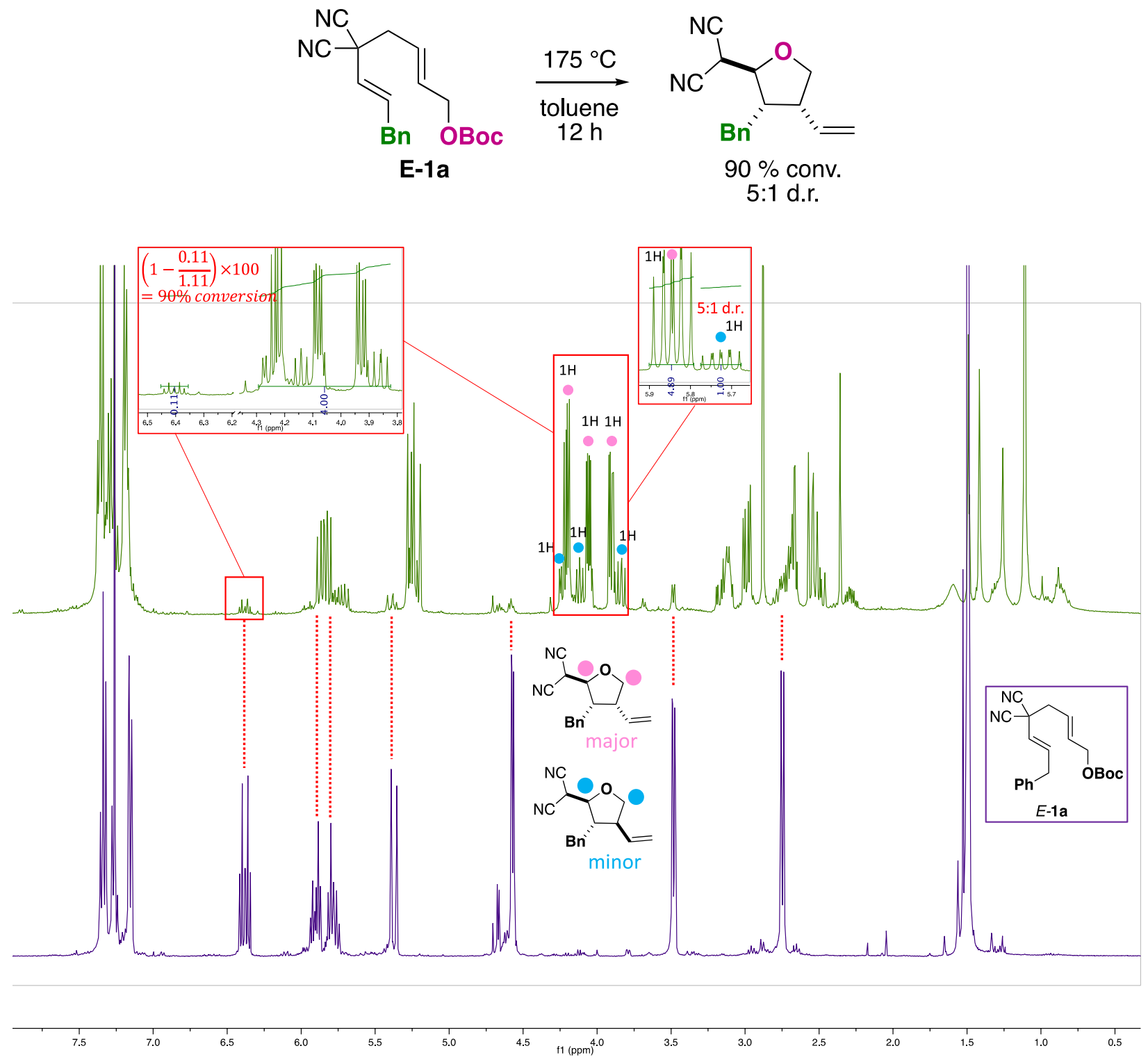


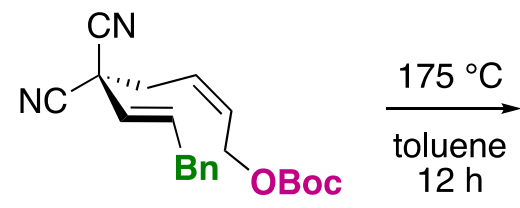

Z-1a

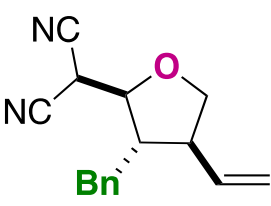

$94 \%$ conv.

10:1 d.r.
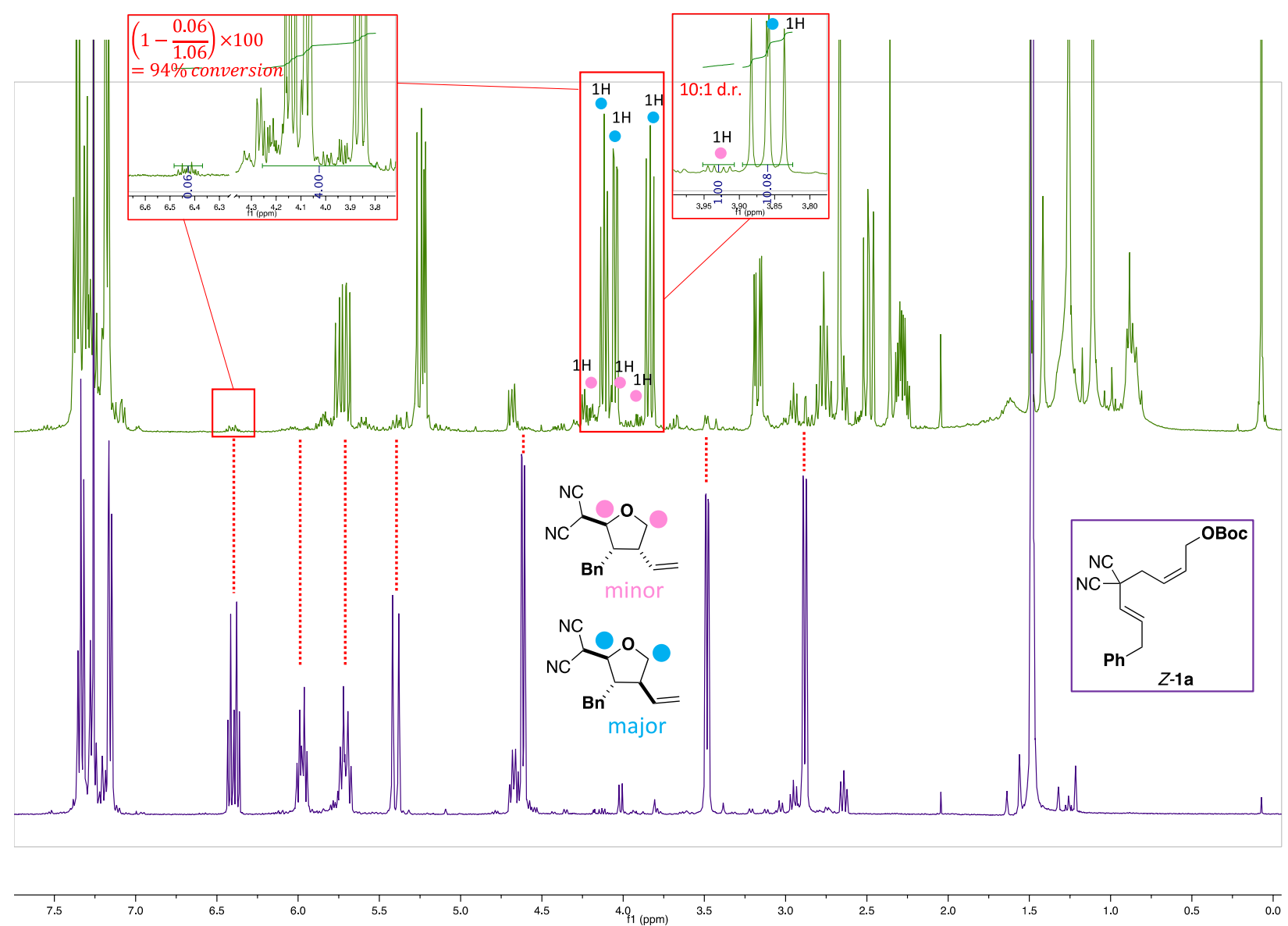
b. Stereochemical outcome of the Cope rearrangement (3 and 4 position)

In order to confirm the stereochemical outcome predicted by the Zimmerman-Traxler model, we performed the following experiment: $E$-1a-OAc was subjected to the Cope rearrangement conditions $\left(175^{\circ} \mathrm{C}, 12 \mathrm{~h}\right.$ in toluene). The expected Cope rearranged product $\mathbf{A}$ was observed (green pathway).

Starting from 3a (made from 1a), we performed a retro-Oxy-Michael addition as depicted in the figure below using $\mathrm{Et}_{3} \mathrm{~N}, \mathrm{Ac}_{2} \mathrm{O}$ in DMF at room temperature. The same product $\mathbf{A}$ was obtained in $60 \%$ (purple pathway). Since both reactions converge to the same product $\mathbf{A}$, we are confident about the assigned stereochemistry of 3a and iso-3a at the 3 and 4 position. (both ${ }^{1} \mathrm{H}$ NMR spectra clearly show that the same product is formed although with slightly different diastereomer ratios).

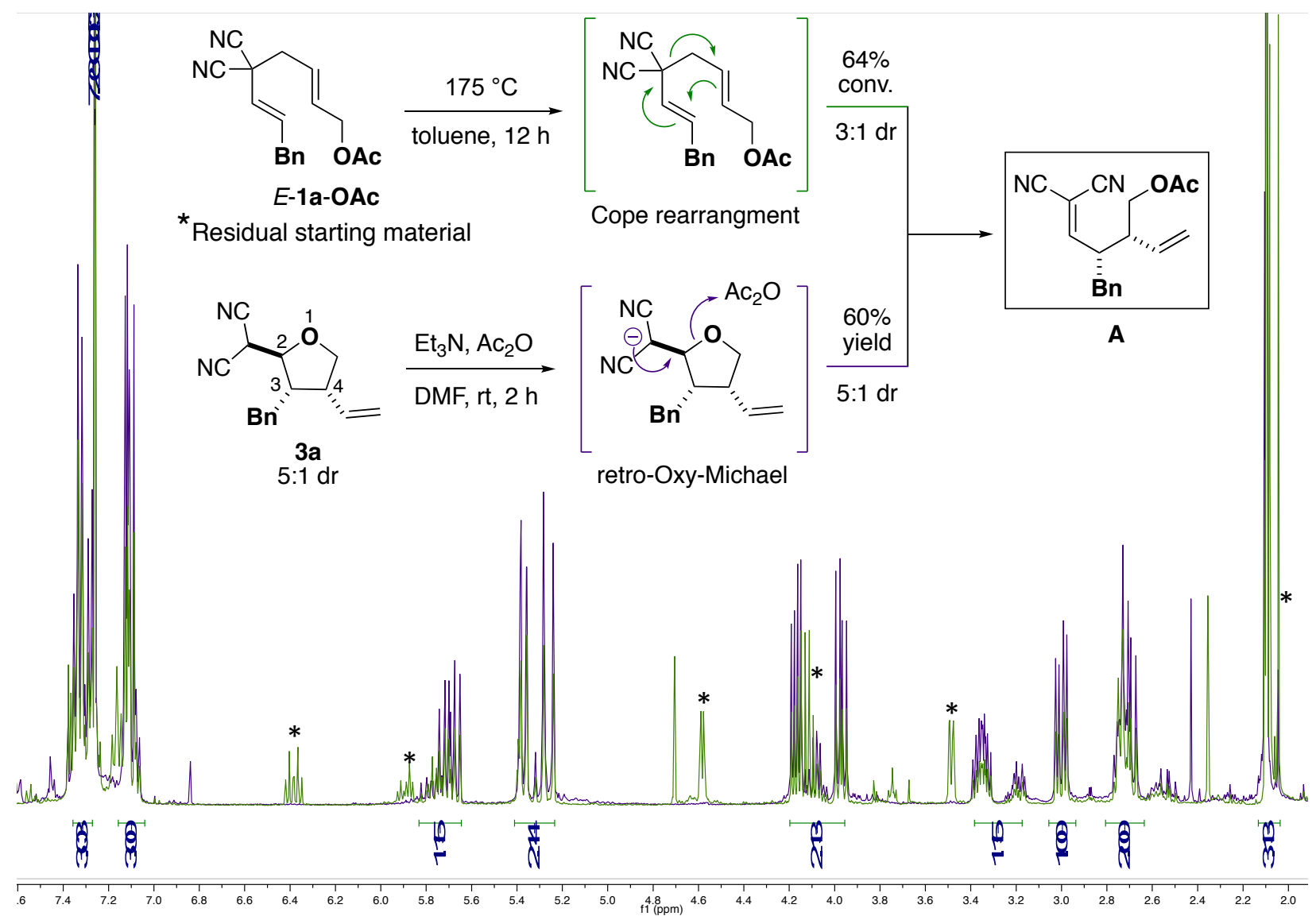

${ }^{13} \mathrm{C}$ NMR comparison of $\mathbf{3 a}$ and $\mathbf{A}$ demonstrate that they can unambiguously and easily be differentiated (please see next figure). 

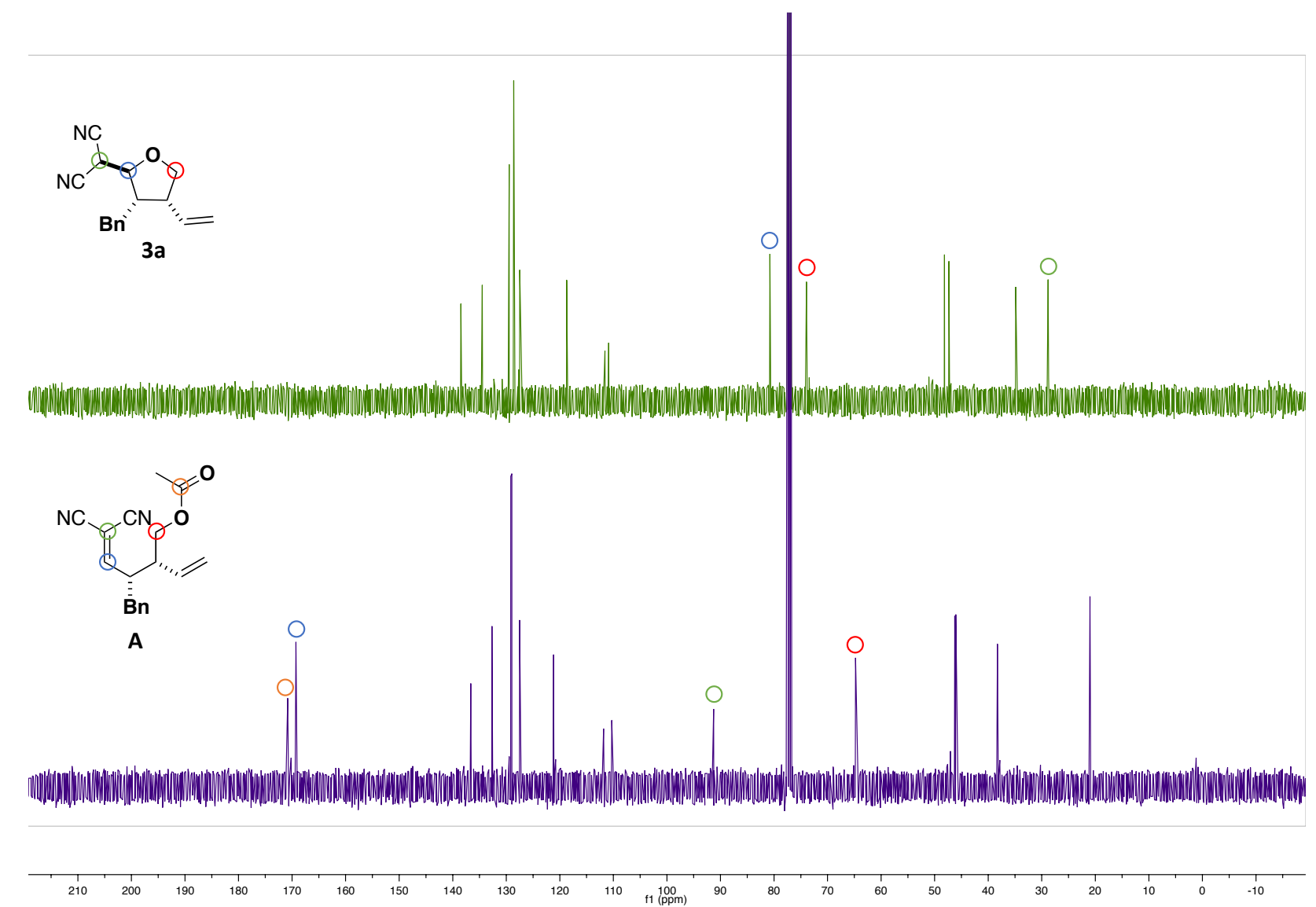

c. Stereochemical outcome of the Cope rearrangement/Oxy-Michael (2 position)

3a and iso-3a were both subjected to the same allylation conditions (described in detail in section 2.d.i.). Then, product 5a and SI-iso-5a were subjected to ring-closing metathesis conditions (described in detail in section 2.d.ii.). While 5a gave the expected product 5b, SI-iso-5a didn't react at all under these conditions and was entirely recovered. In this system, only one diastereomer, presenting the appropriate geometry, can react. This experiment allowed us to confirm the assigned stereochemistry of $\mathbf{3 a}$ and iso-3a at the 2 position.<smiles>C=C[C@@H]1CO[C@H](C(C#N)C#N)[C@@H]1Br</smiles><smiles>C=CC1COC(C(C#N)C#N)C1Br</smiles>
iso-3a

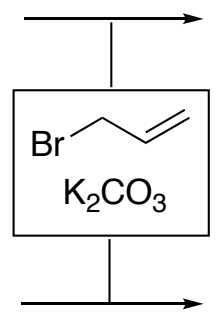

5a, 8:1 dr

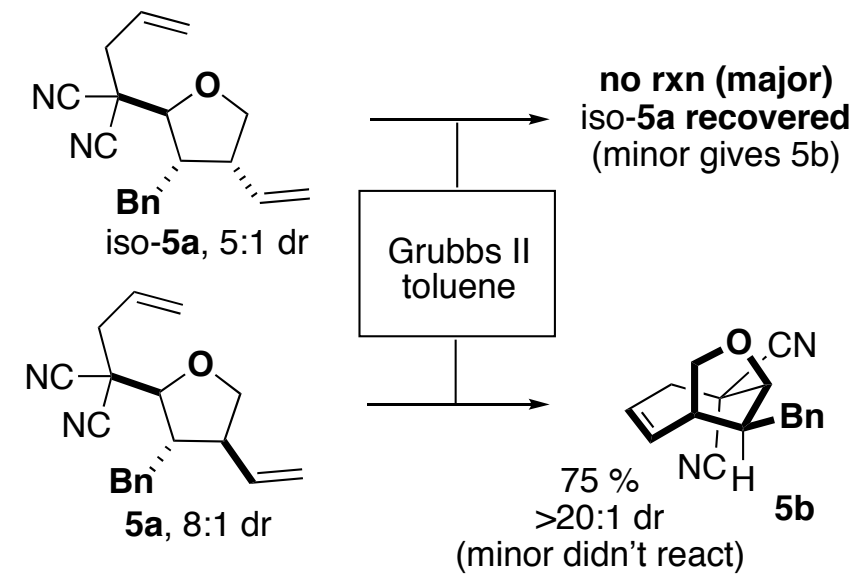




\section{References}

(1) Takemoto, S.; Ishii, T.; Yasuda, S.; Ohmiya, H. Synergistic N-Heterocyclic Carbene/PalladiumCatalyzed Allylation of Aldehydes with Allylic Carbonates Bull. Chem. Soc. Jpn. 2019, 92(5), 937-940.

(2) Wang, L.; Li, P.; Menche, D. Angew. Chem. Int. Ed. 2010, 49, 9270 -9273.

6. ${ }^{1} \mathrm{H} \&{ }^{13} \mathrm{C}$ NMR spectra 
${ }^{1} \mathrm{H} \&{ }^{13} \mathrm{C}$ NMR

spectra 


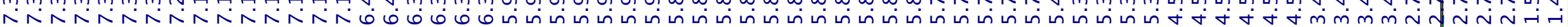
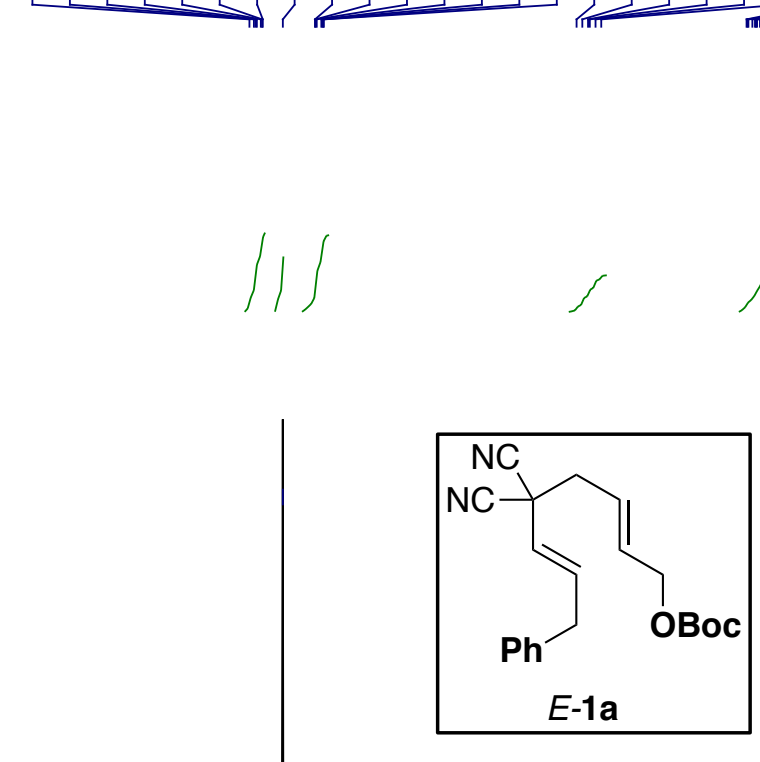


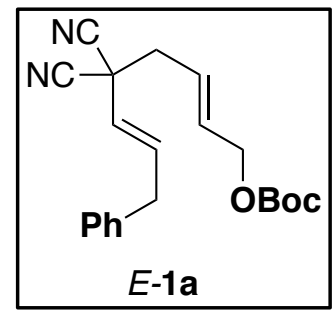

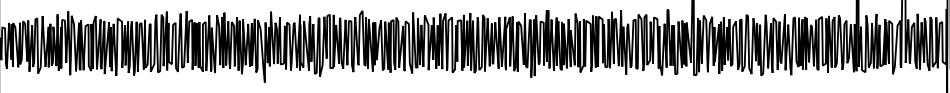

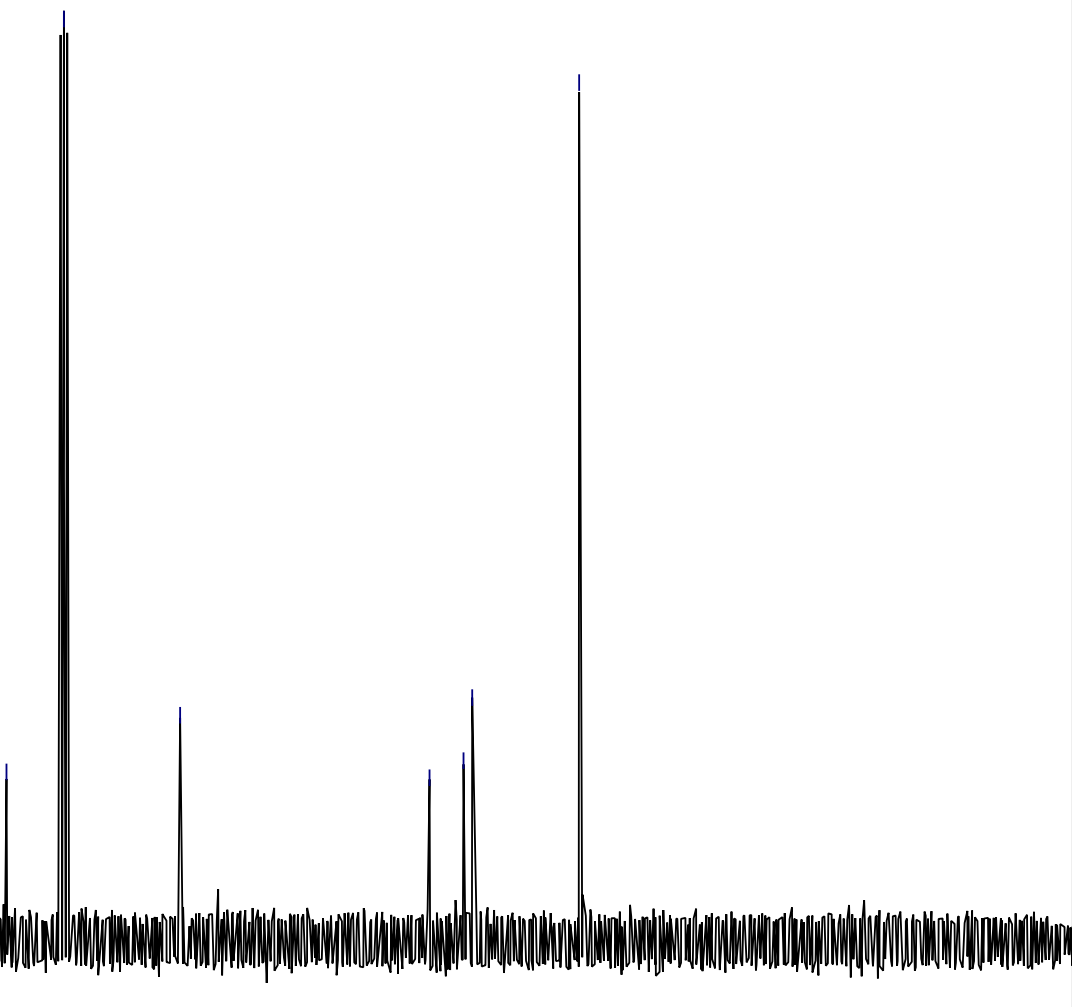



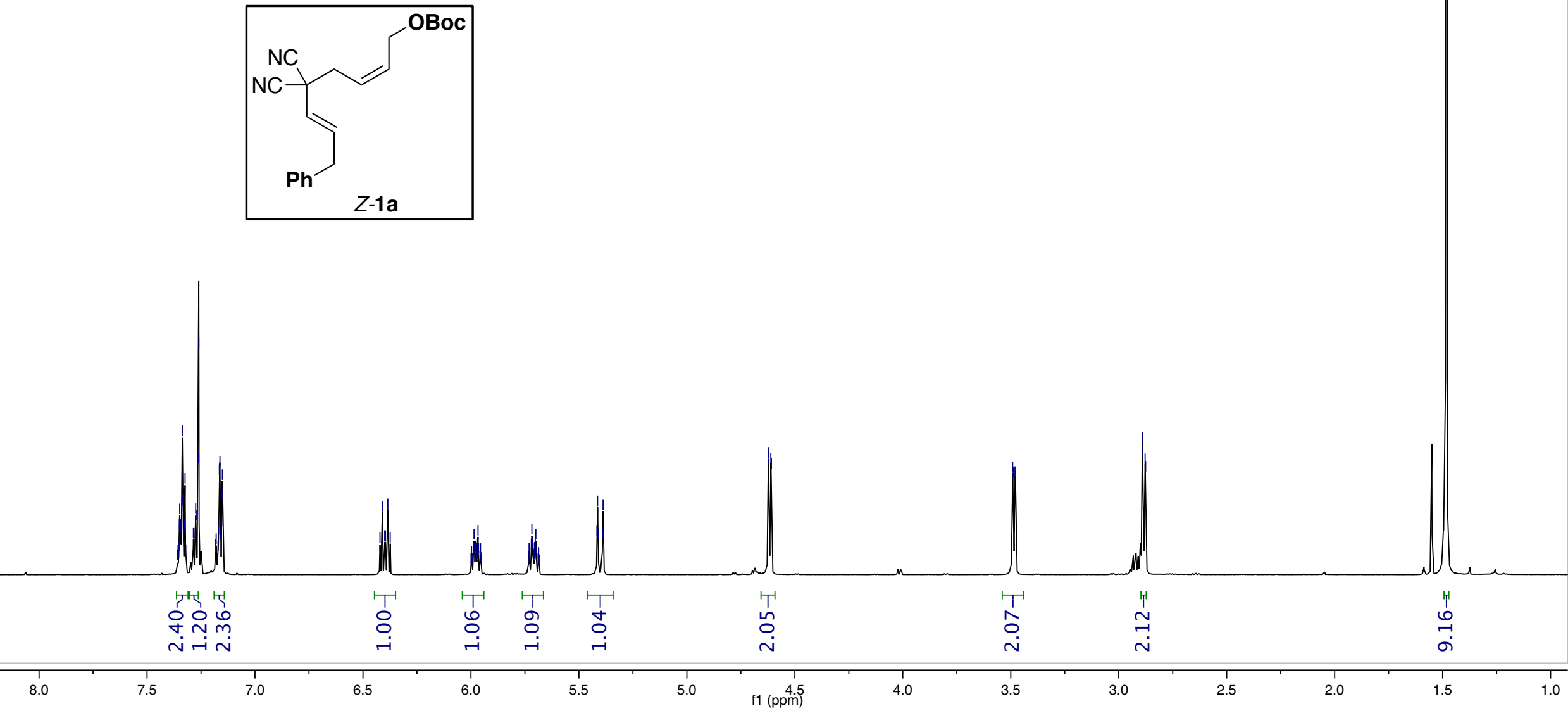


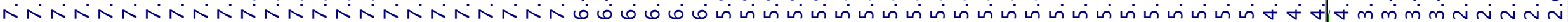
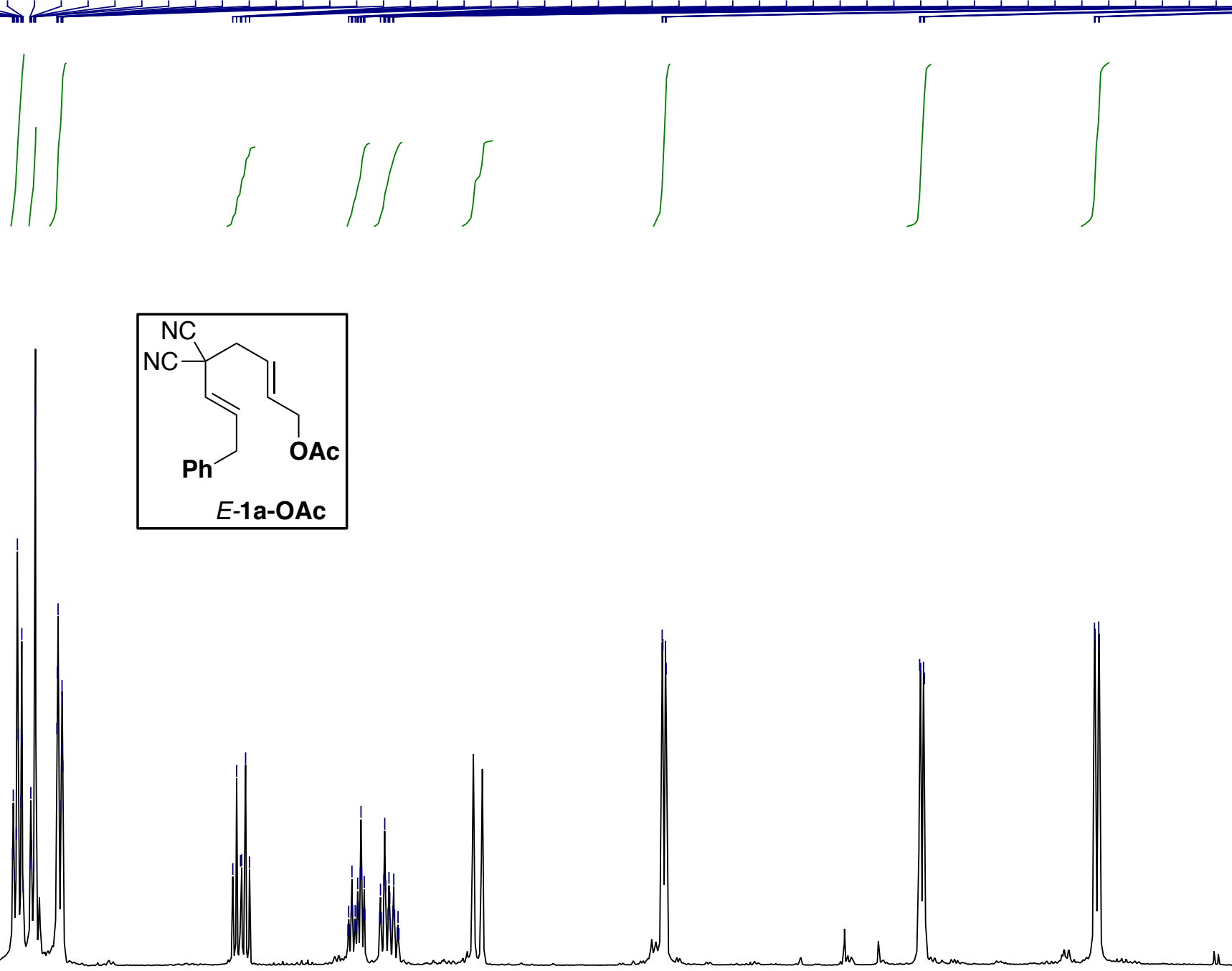

\section{श्साप्र}

ㄷำ

i $\sim \dot{\sim}$

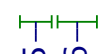

กิ

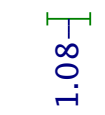

T
¿

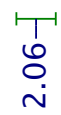

4.5




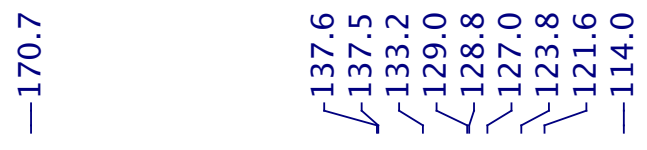

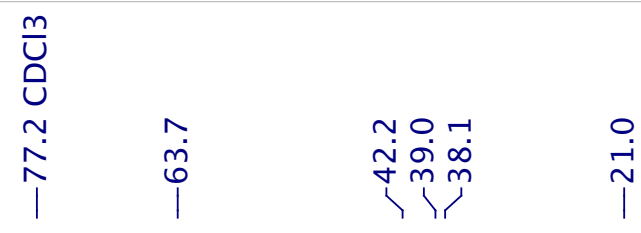

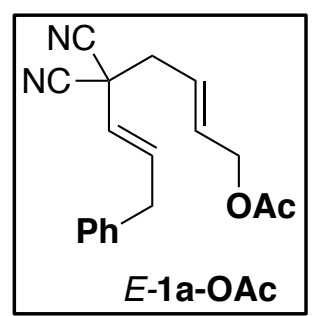

$210 \quad 200$ 


\section{$\stackrel{m}{\mathrm{U}}$}



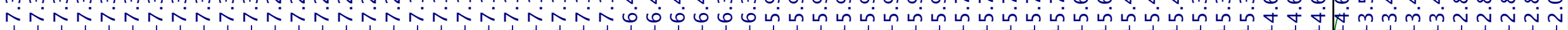
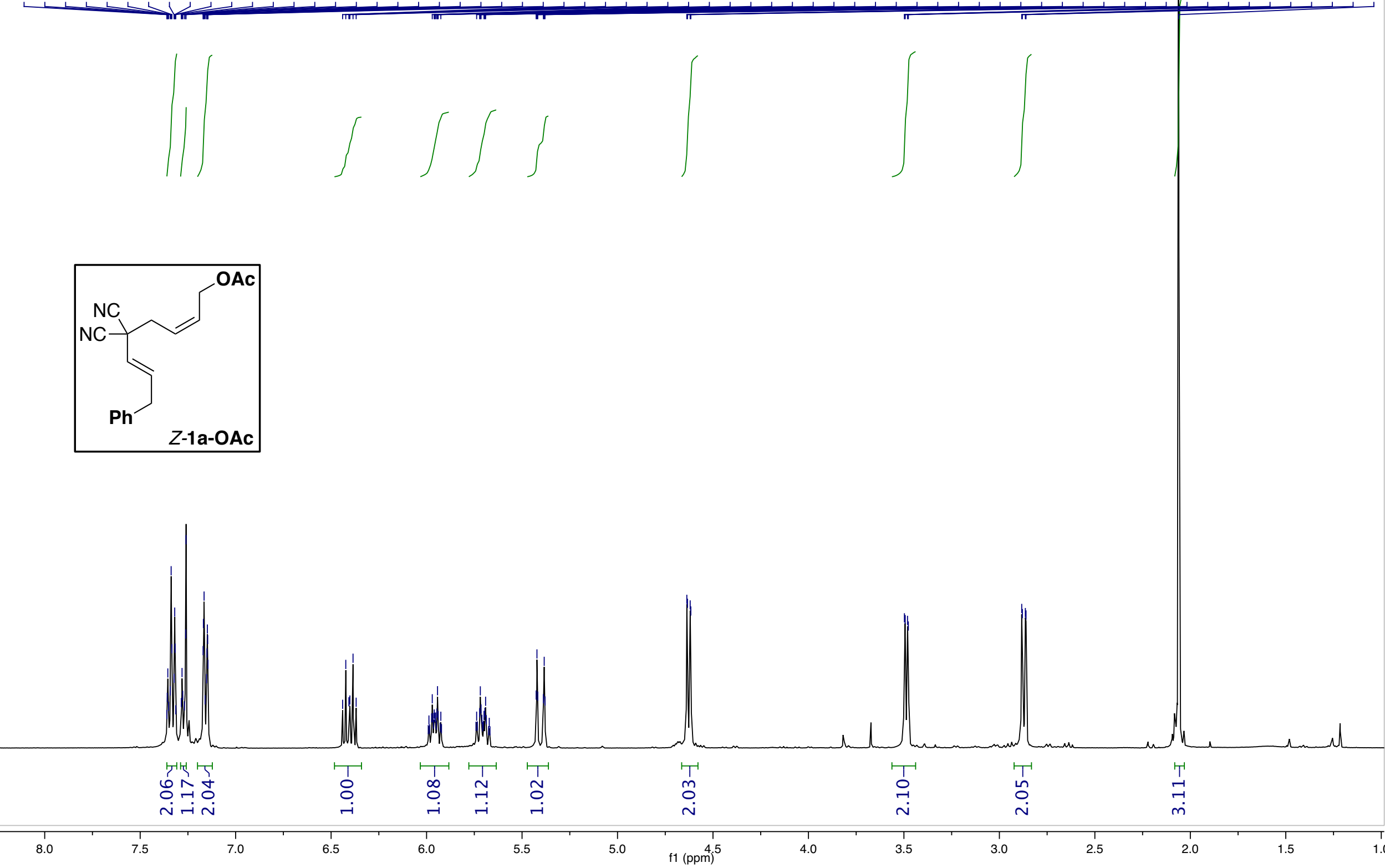


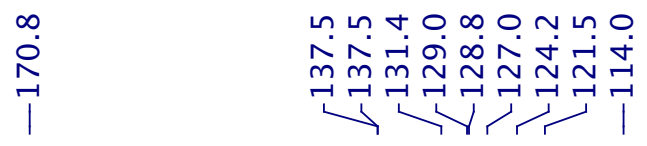

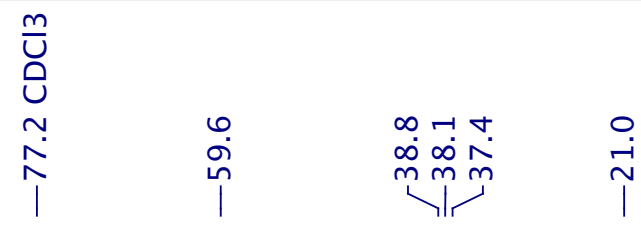
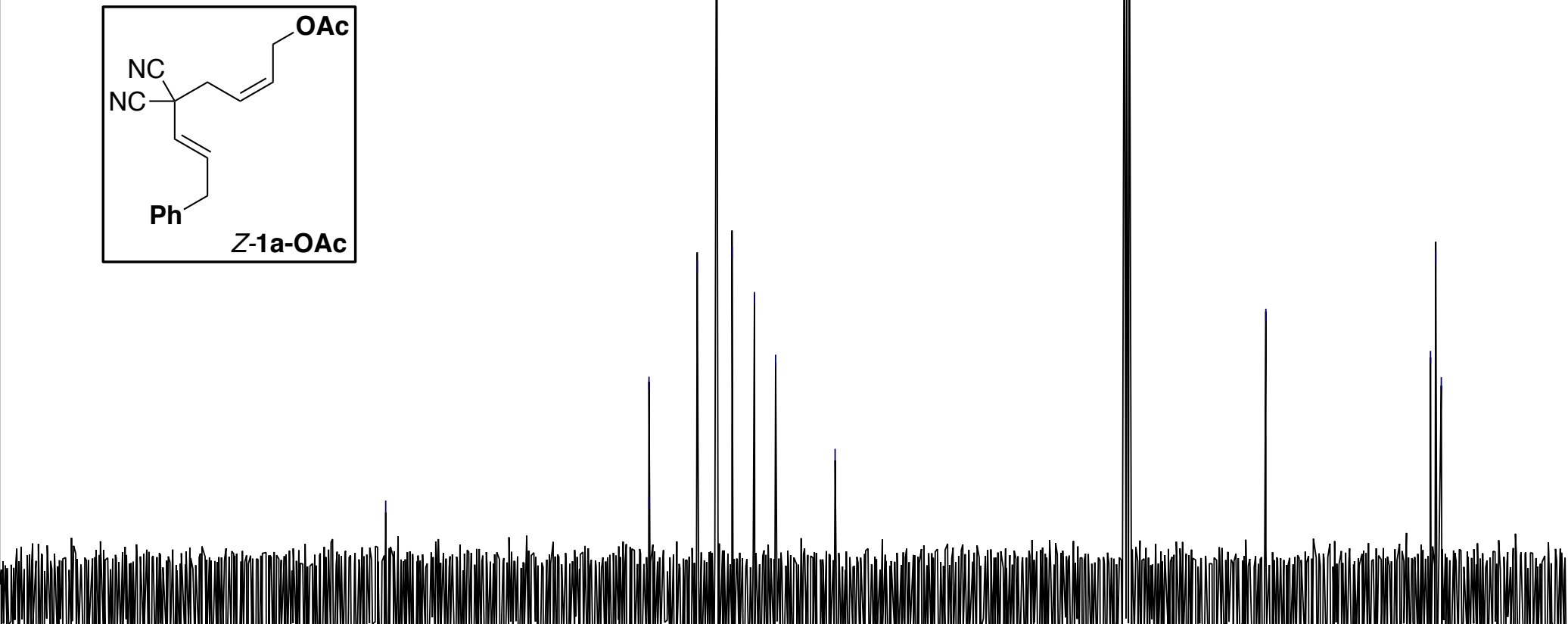

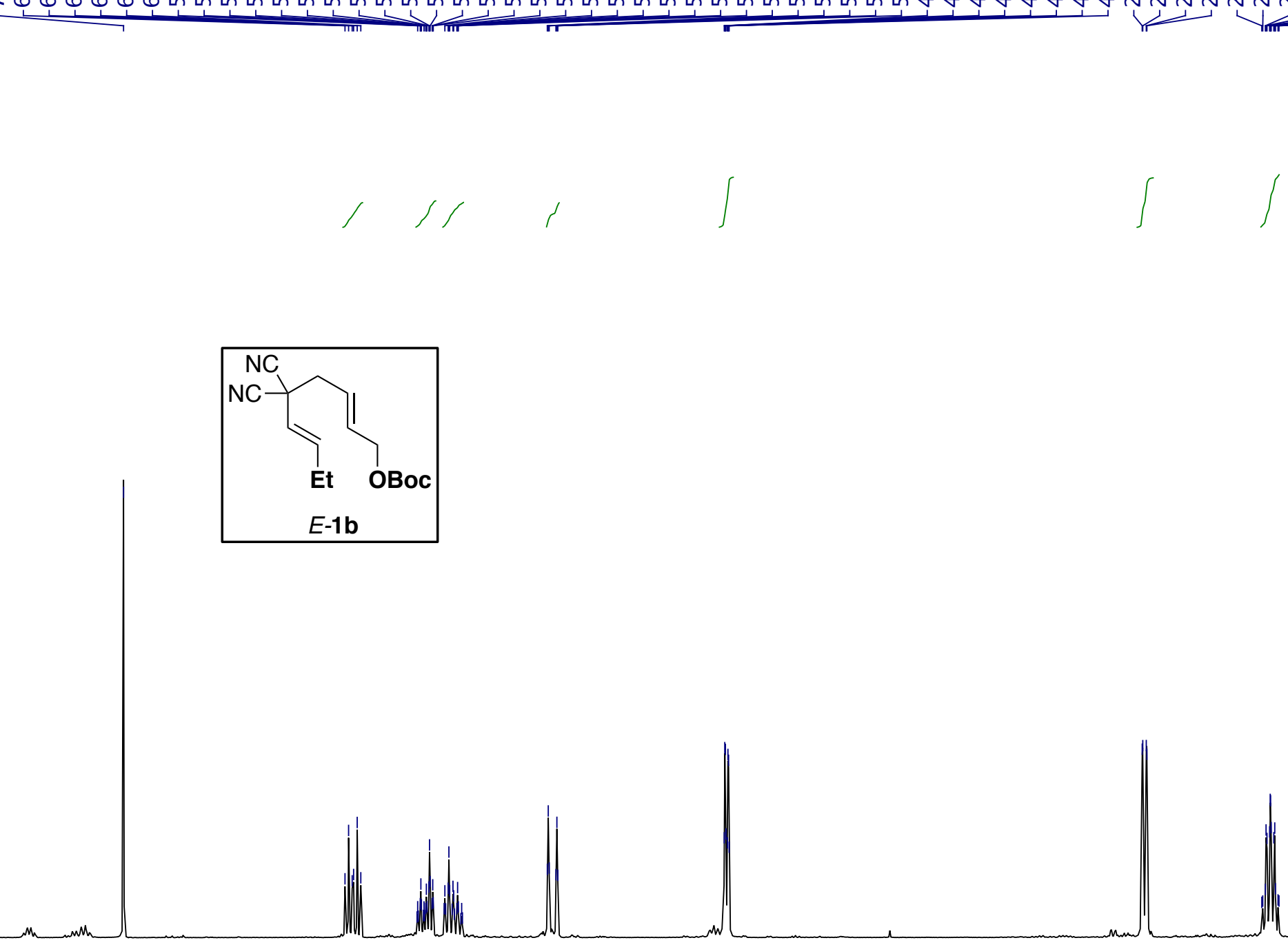

Tा

$\begin{array}{lll}1 & 1 \\ 0 & 0 & 0 \\ 0 & -1 & -1\end{array}$

T
no
n

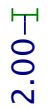

ำ

4.5

$\mathrm{f} 1(\mathrm{ppm})^{4.0}$ 


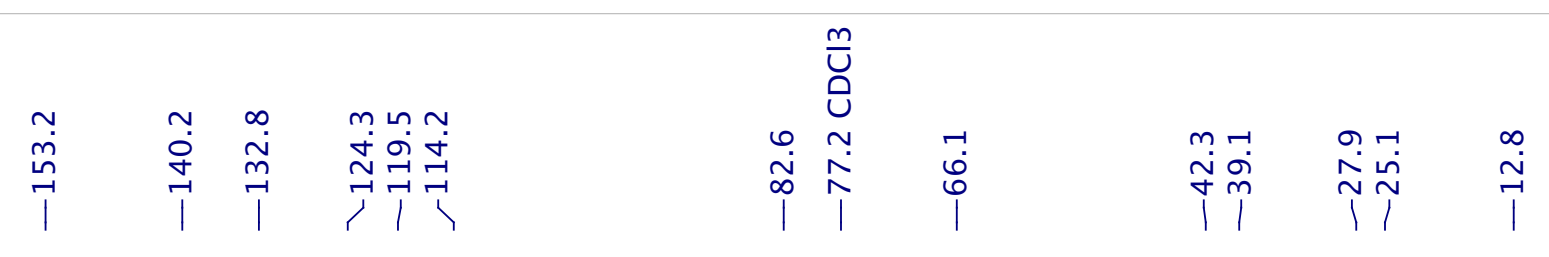

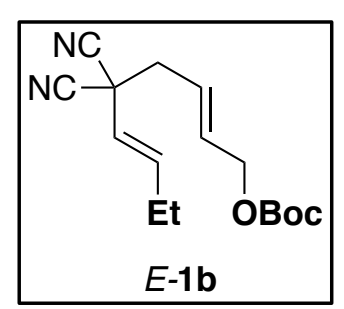

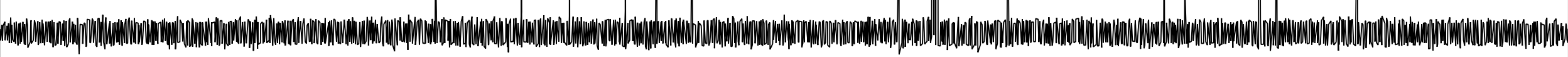



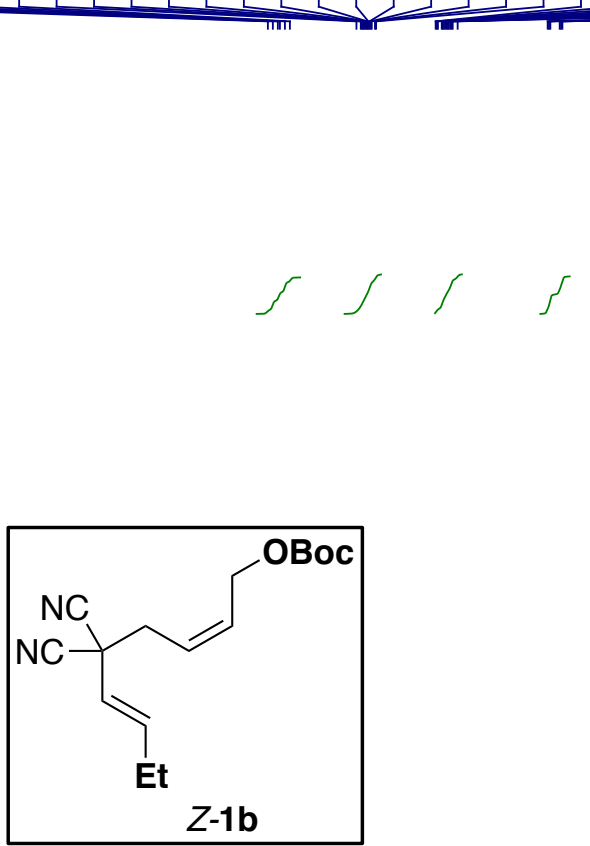
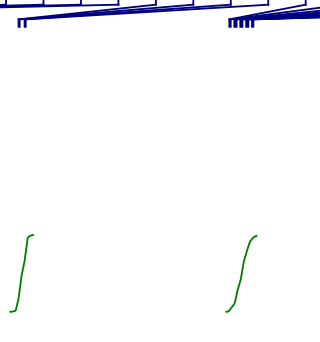


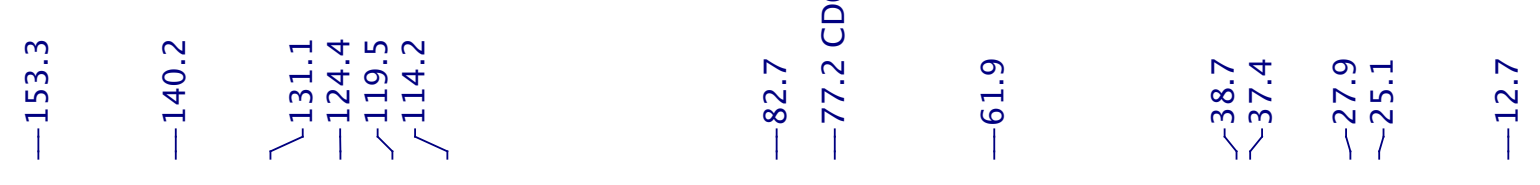

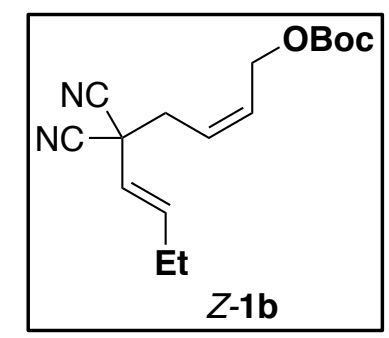

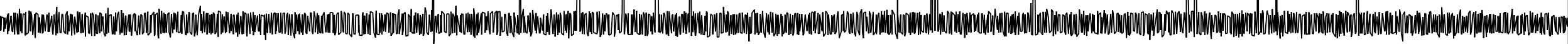

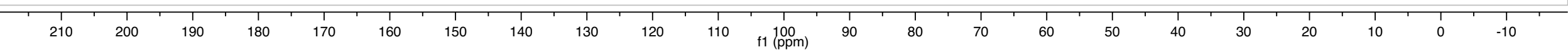




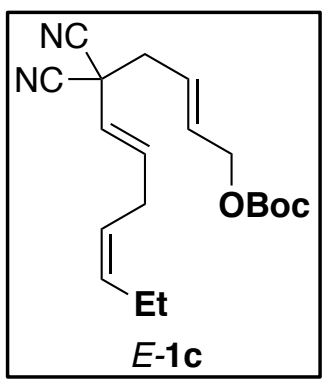




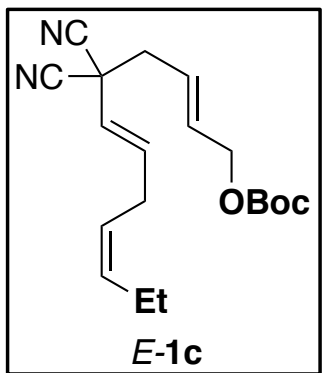



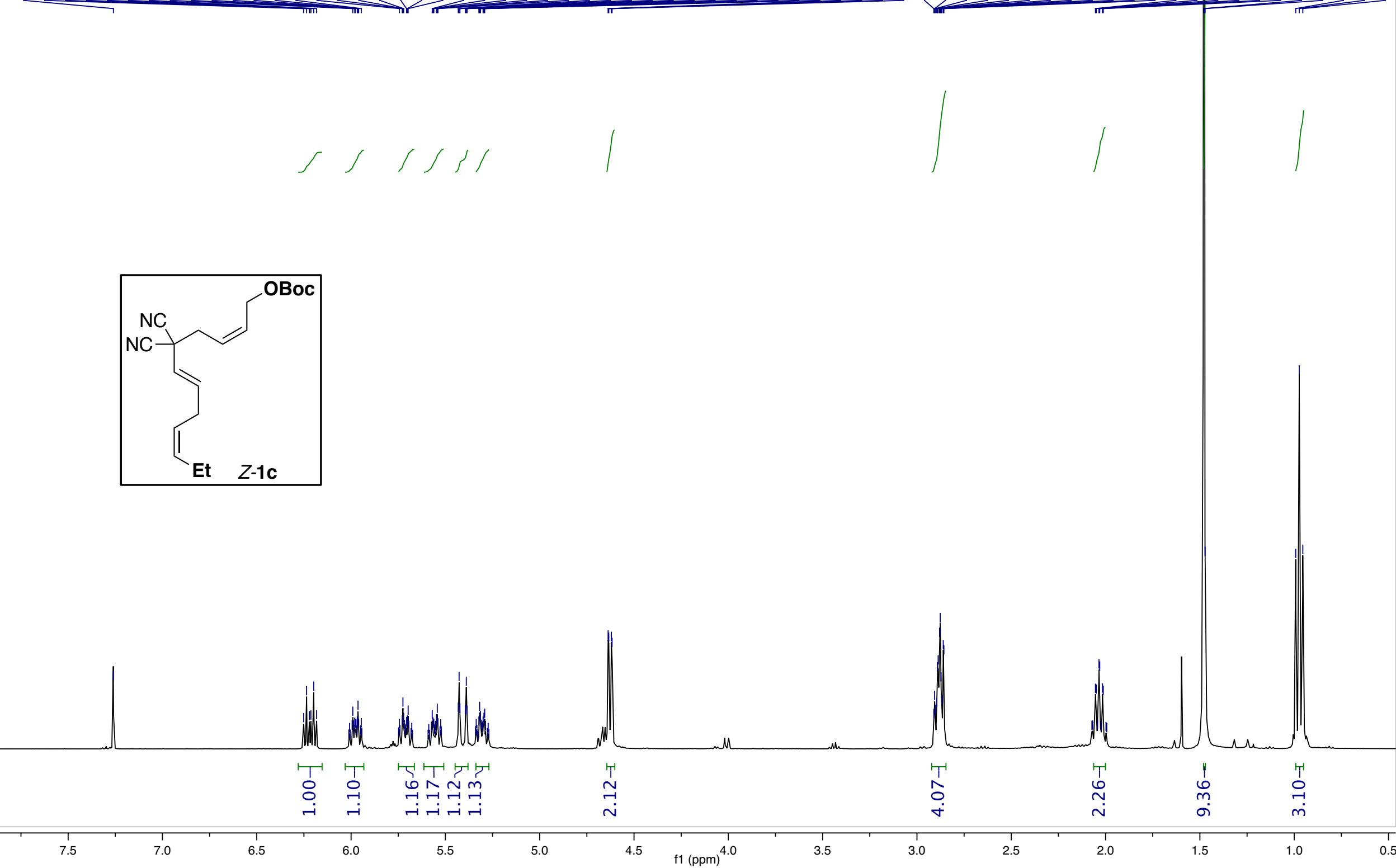


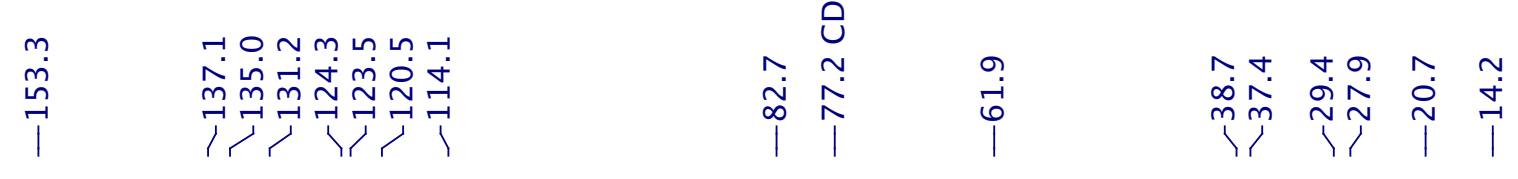
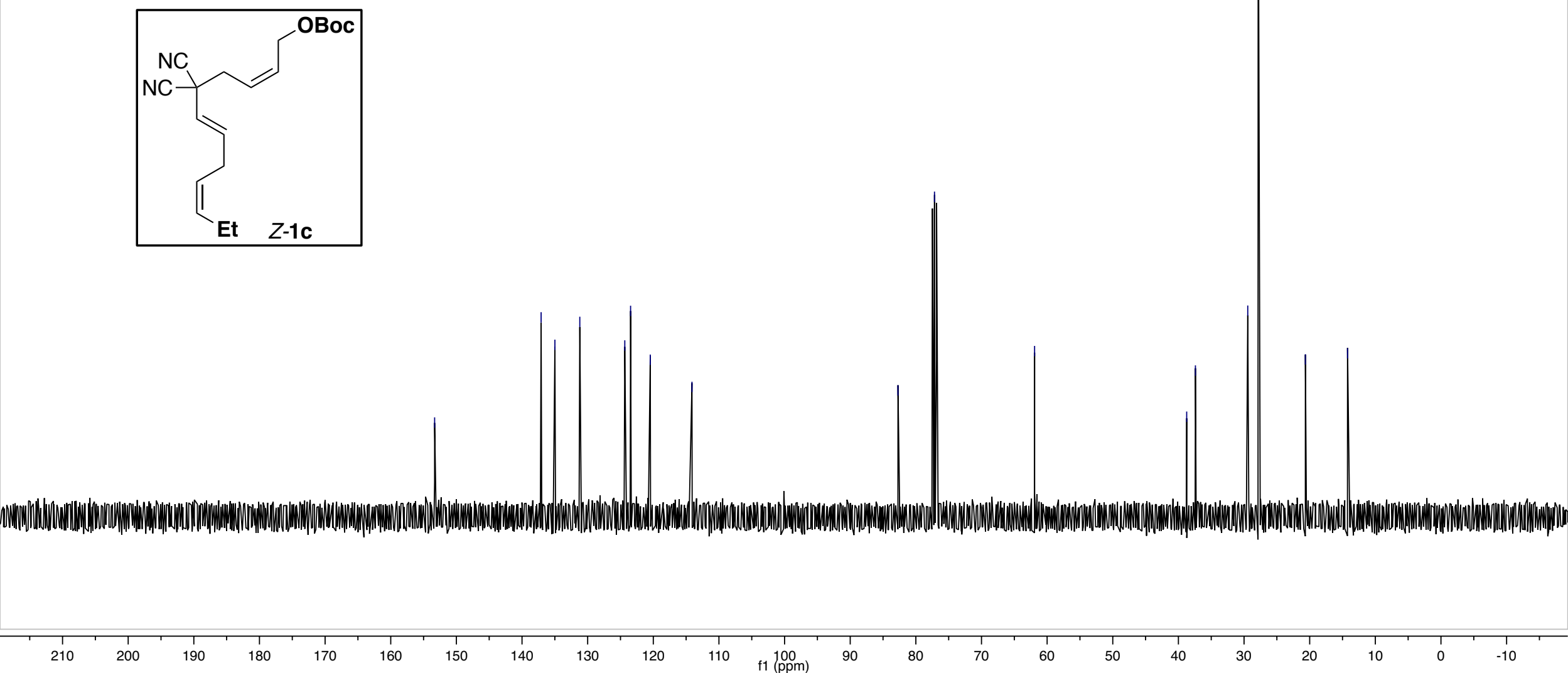

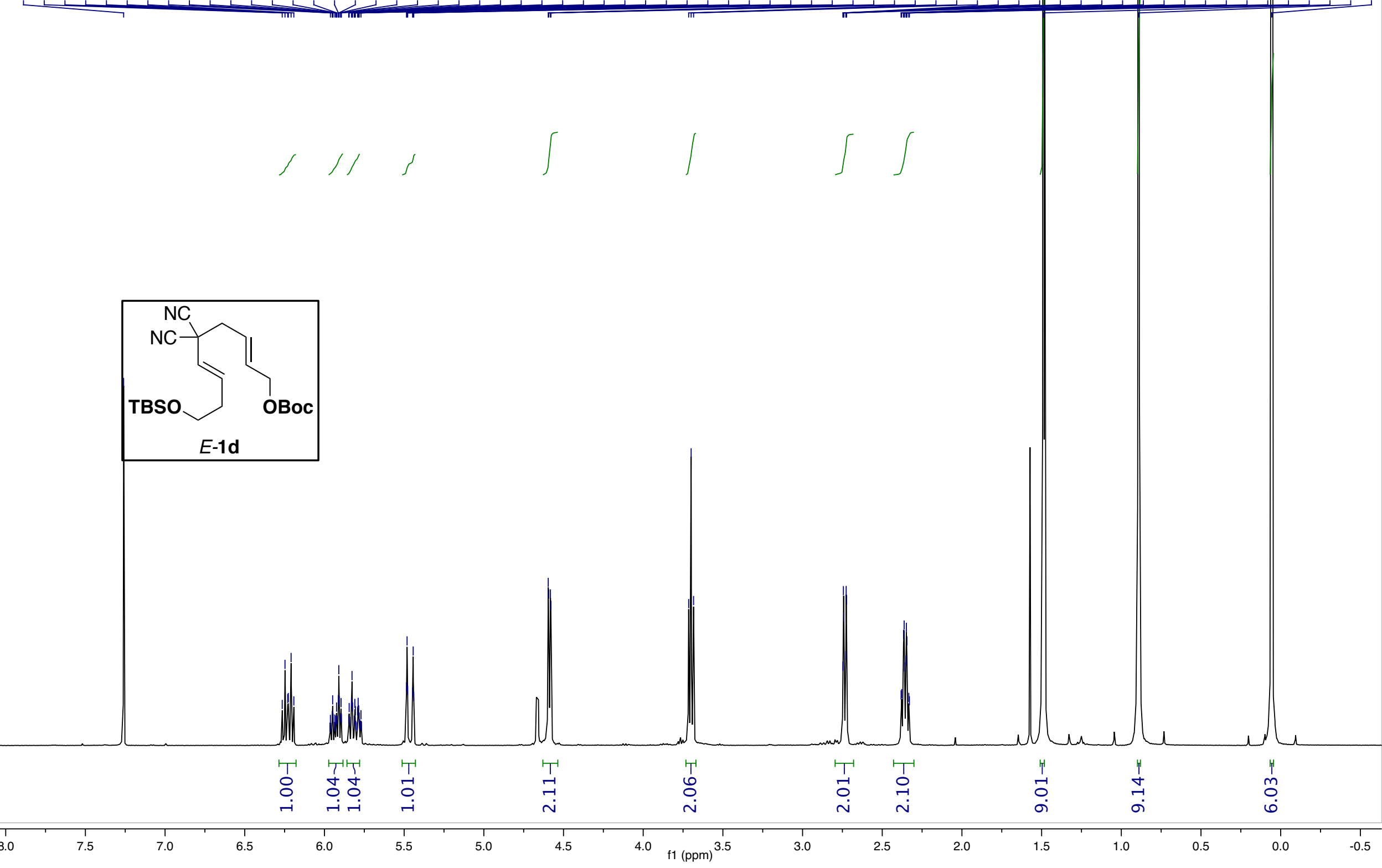


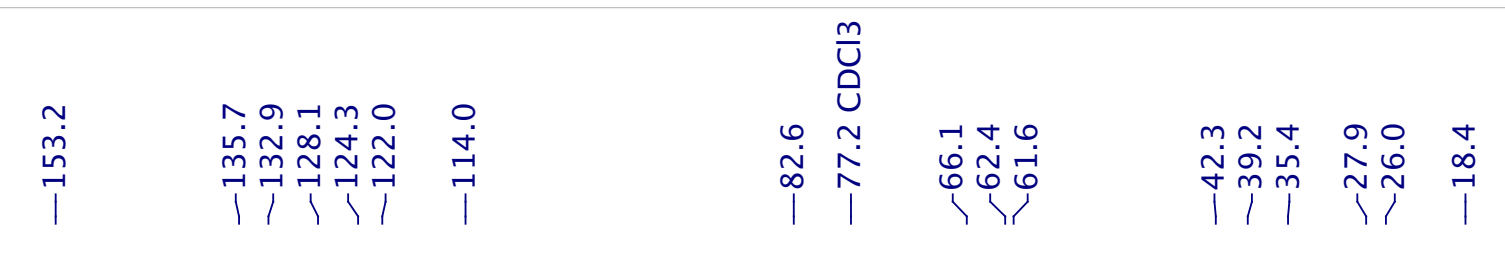
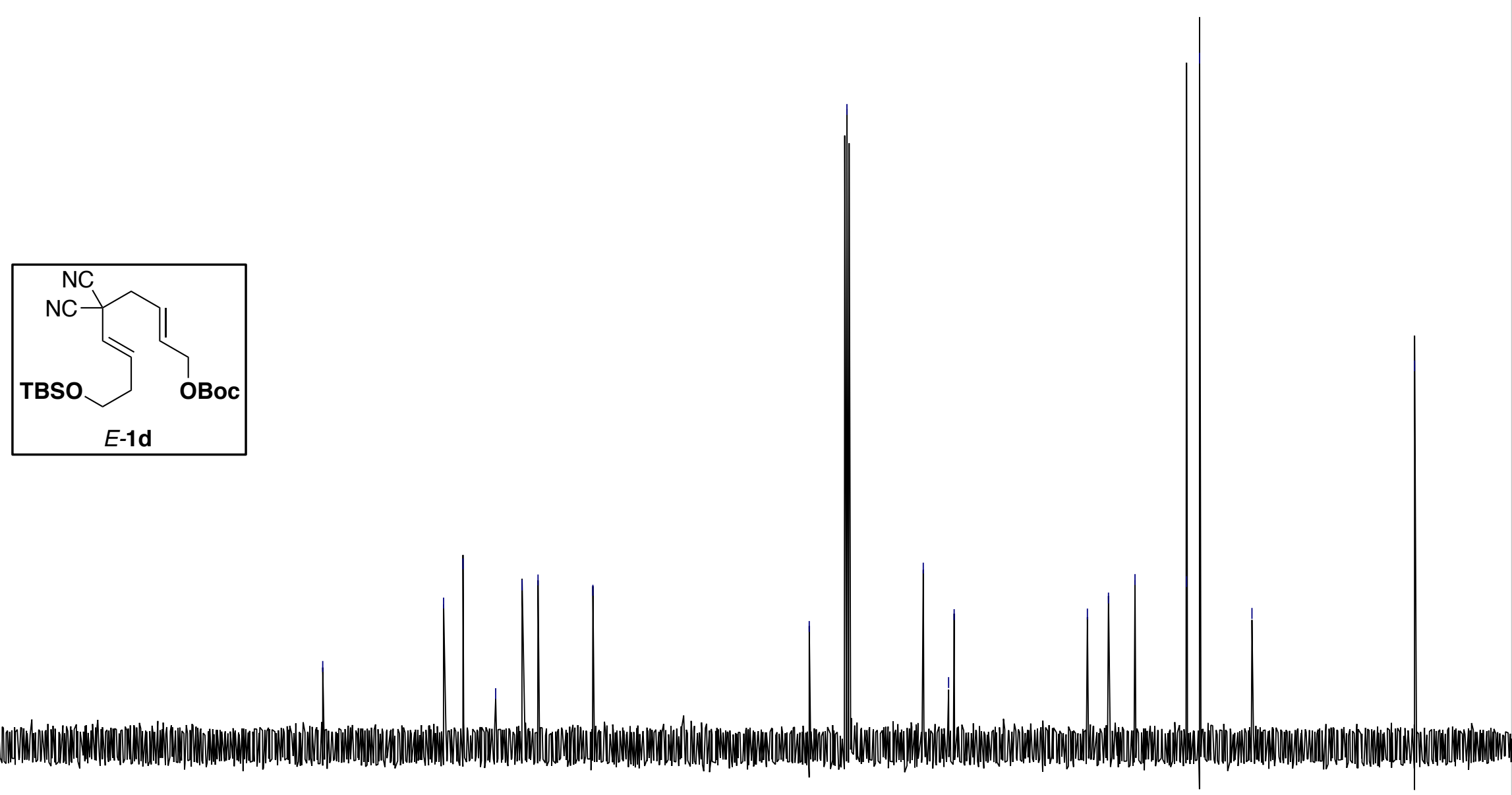

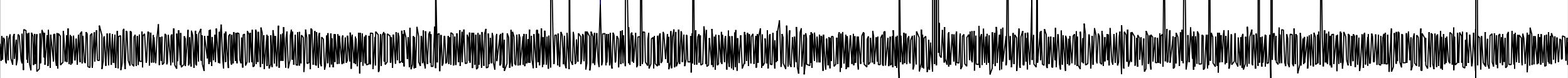

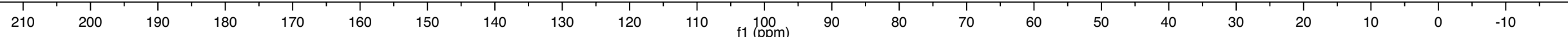




\section{* Trace of Knoevenagel starting material}

$\frac{m}{\mathrm{U}}$

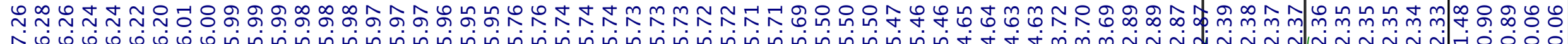

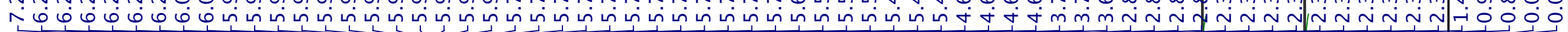
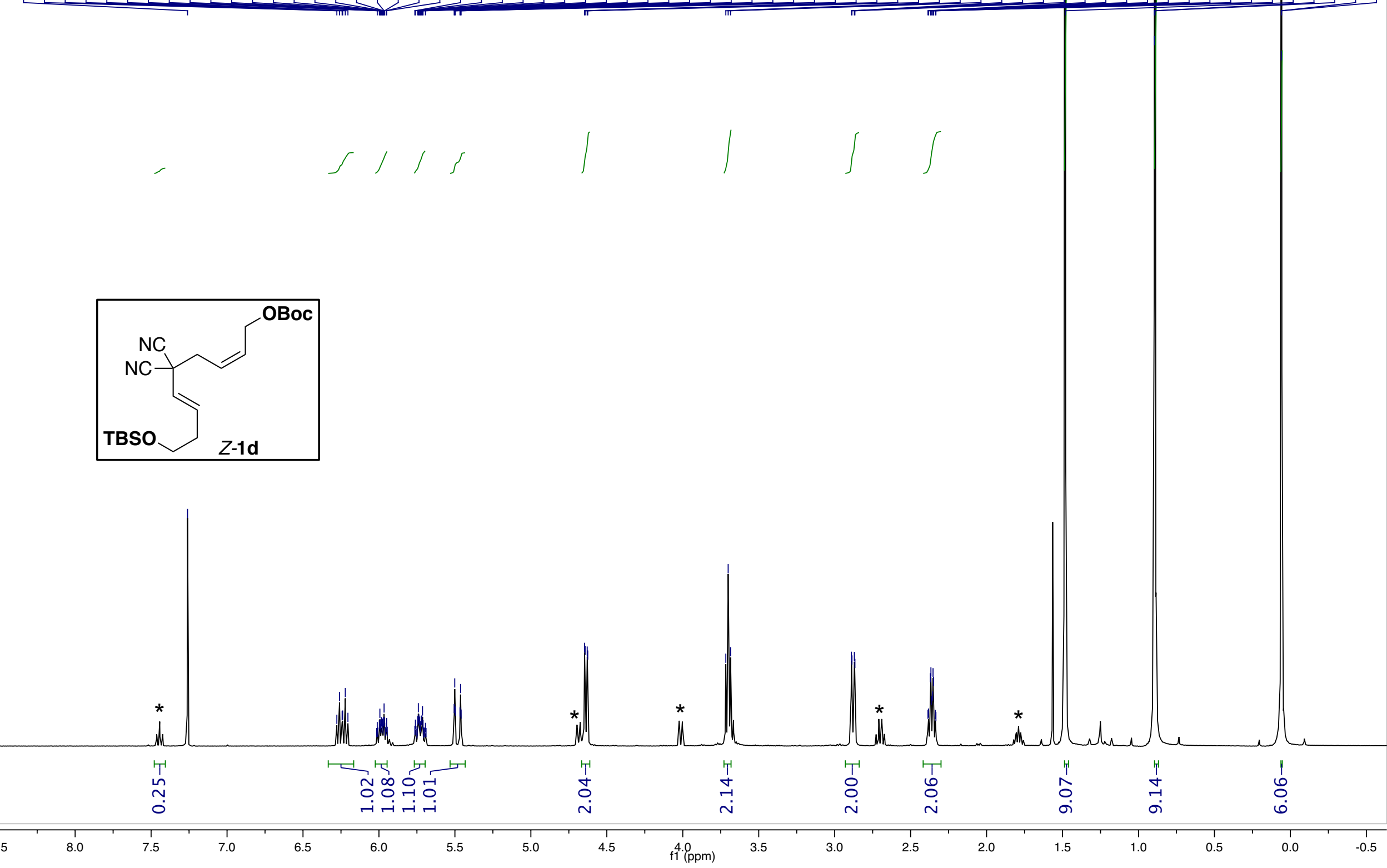


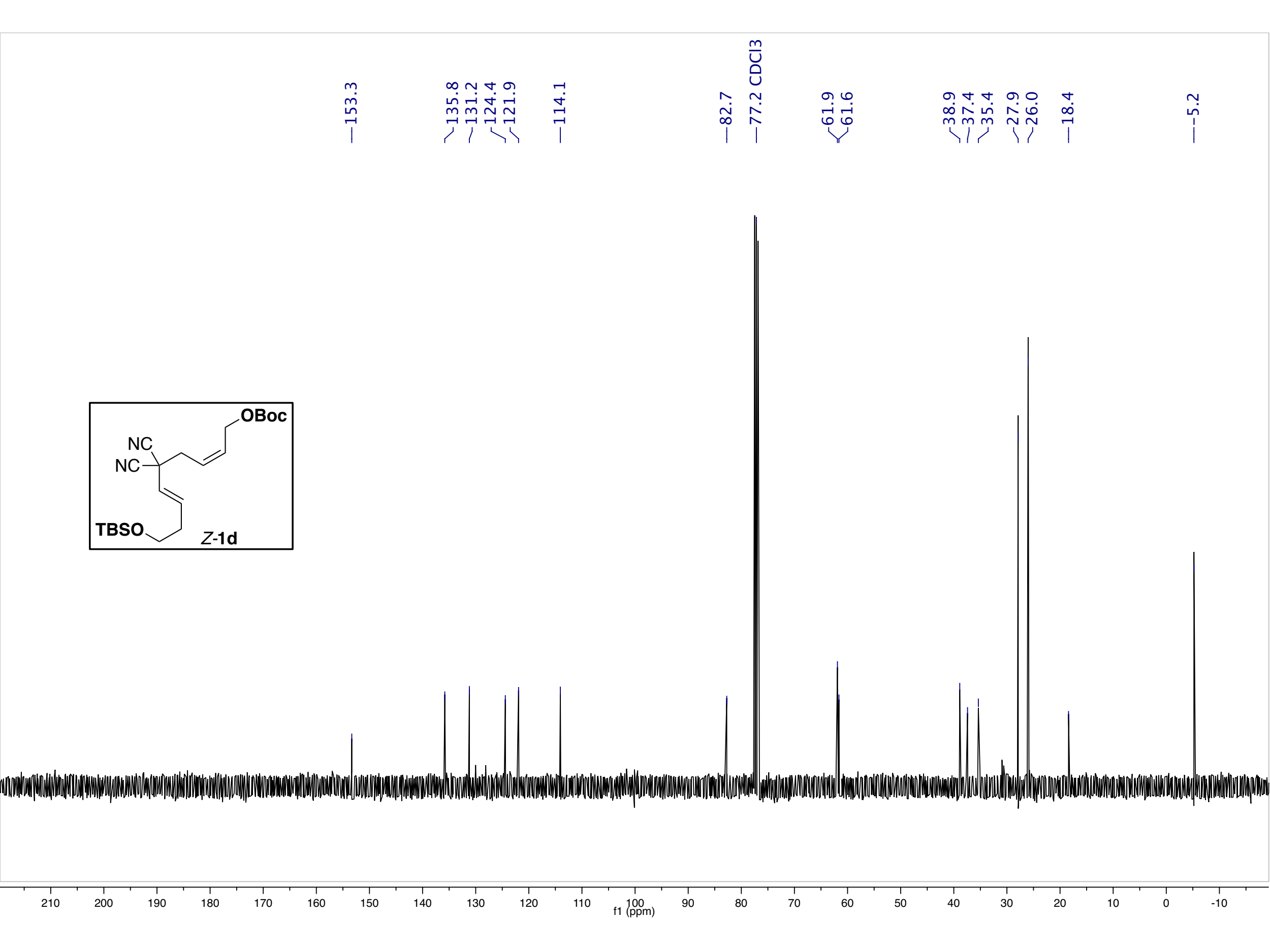


$\underline{\underline{U}}$

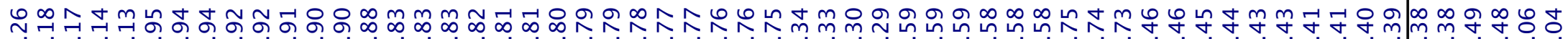
ヘ
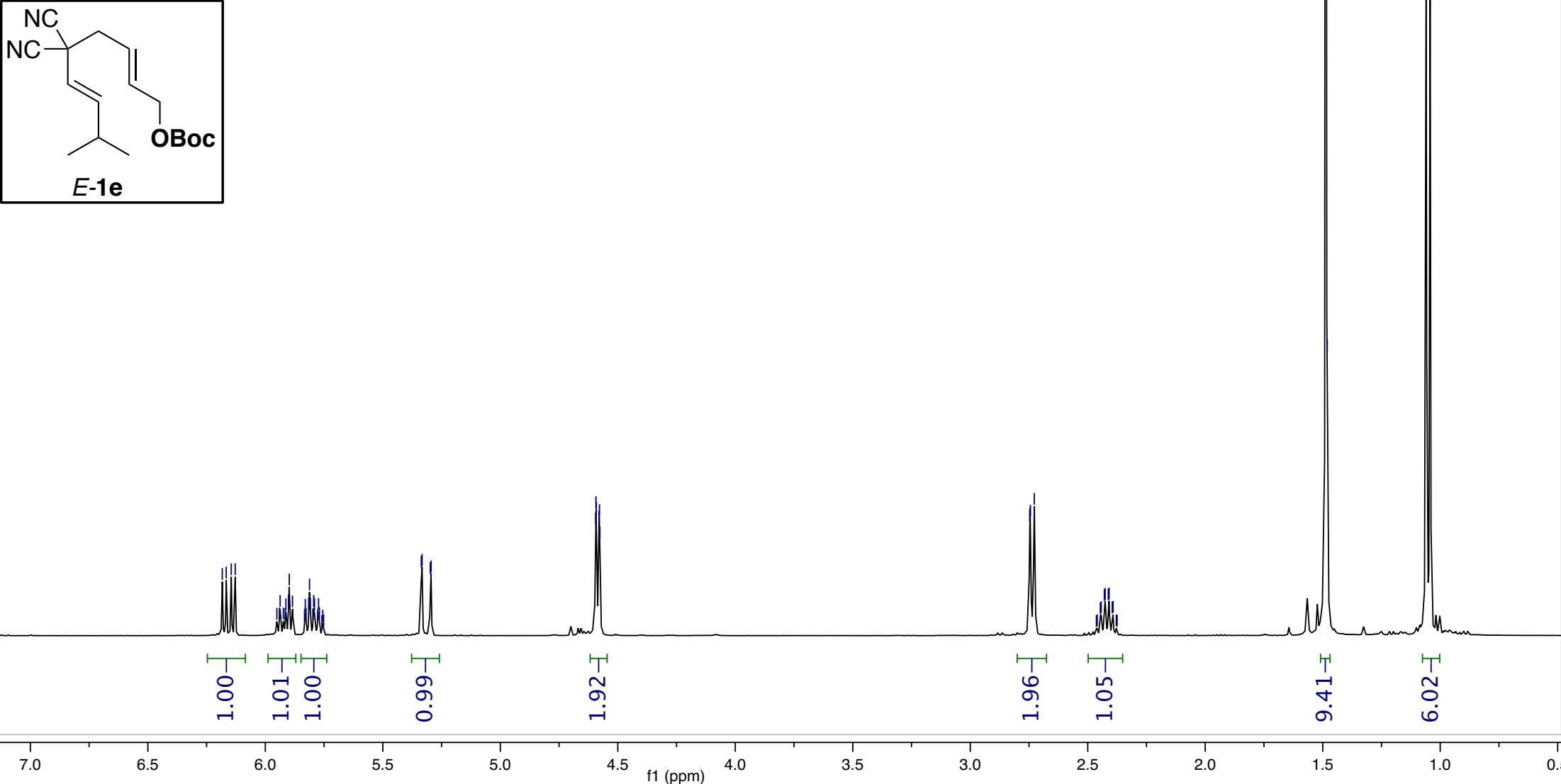


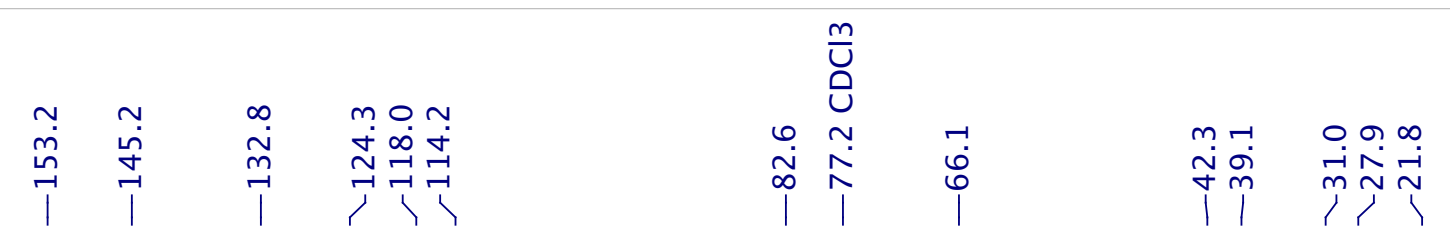
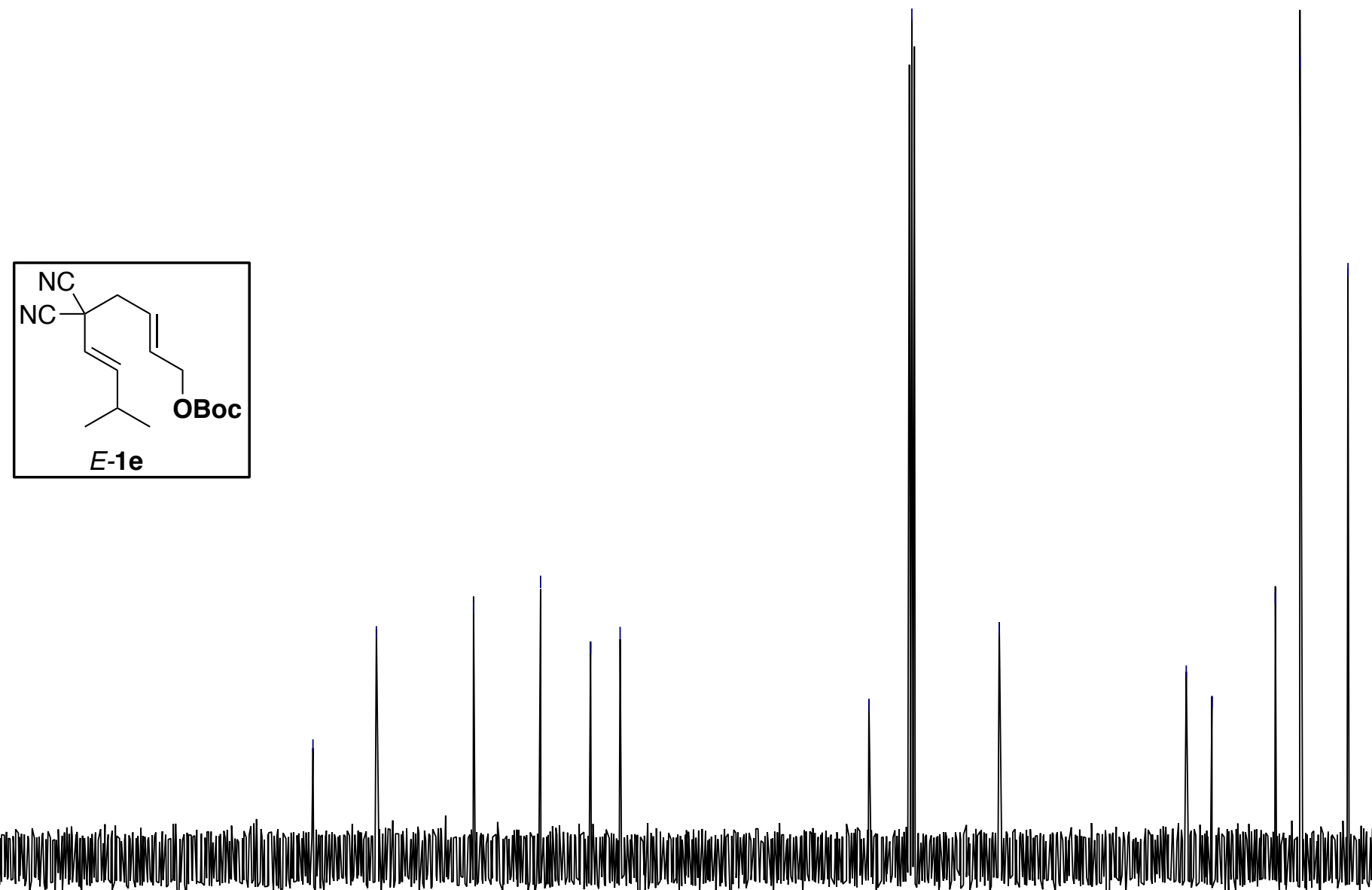

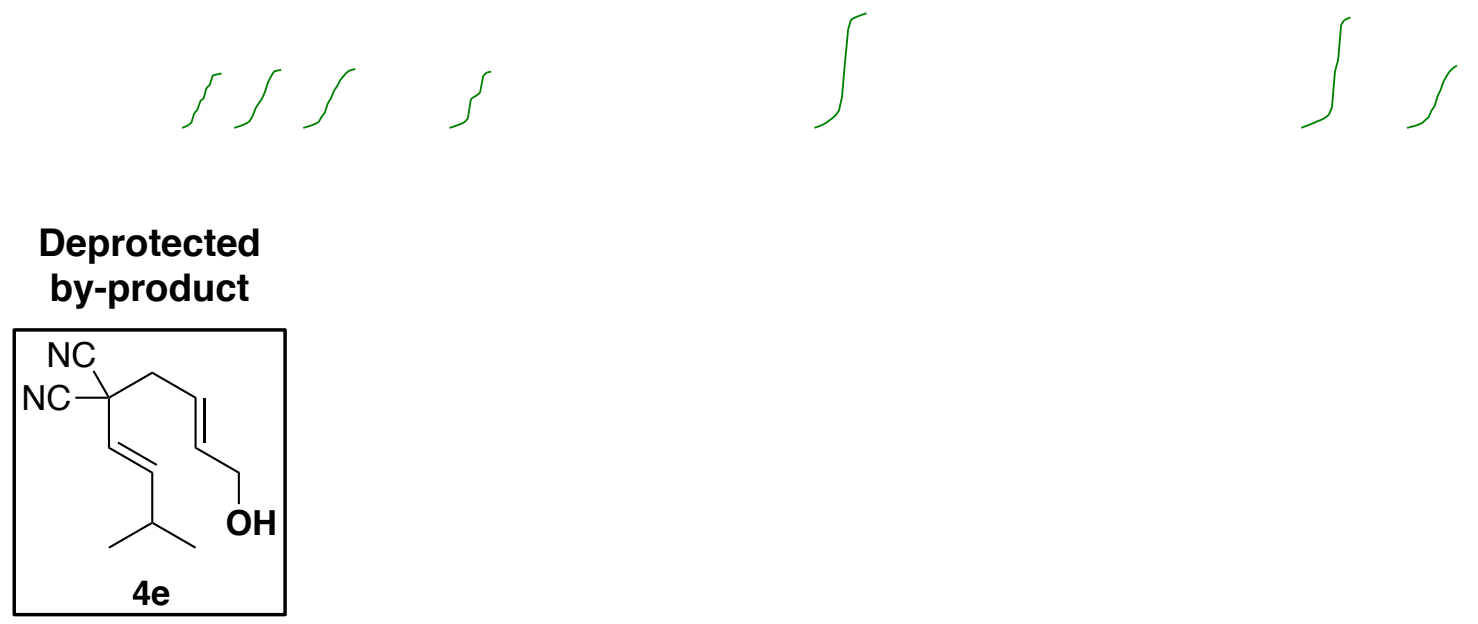

\section{Deprotected}

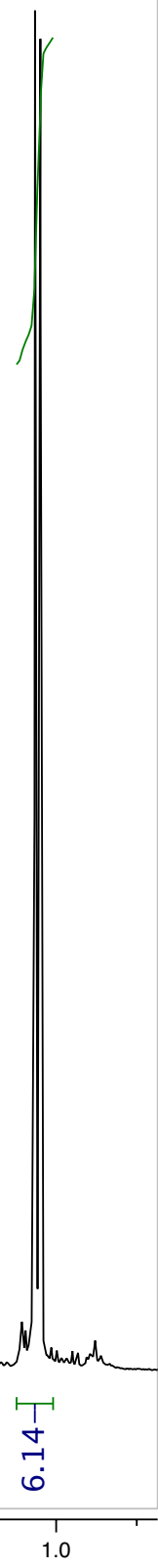



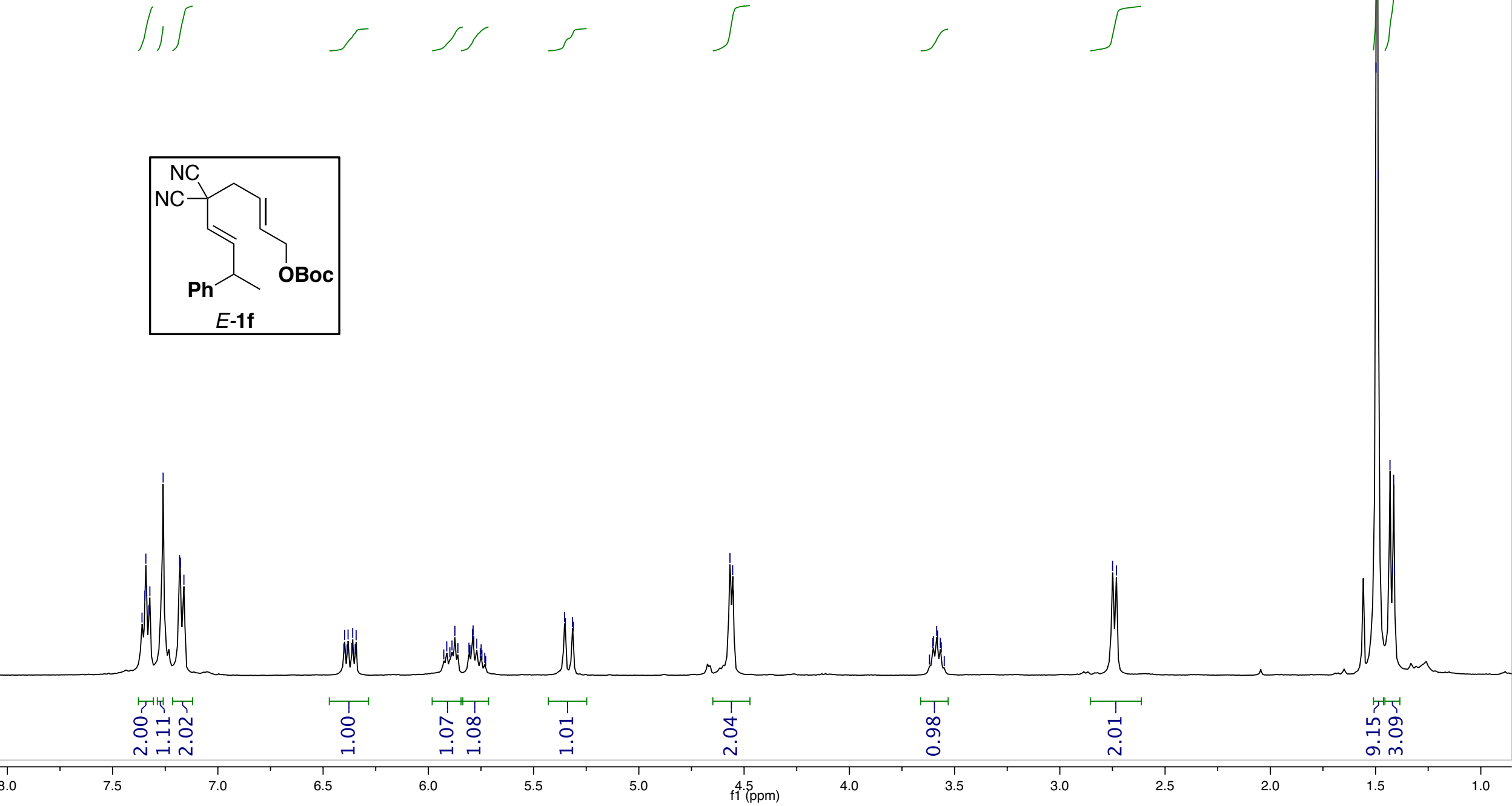

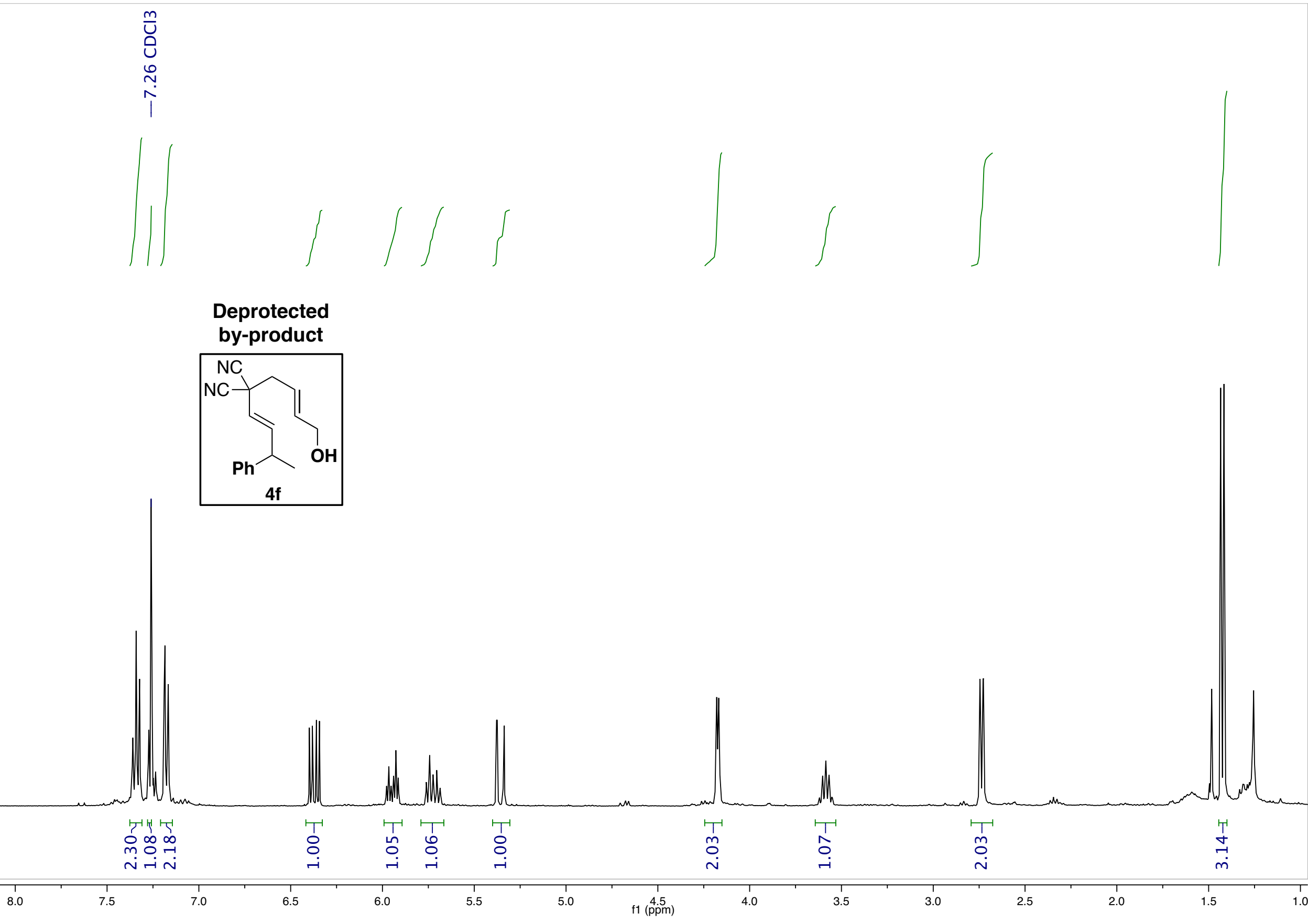


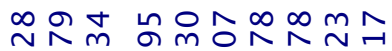

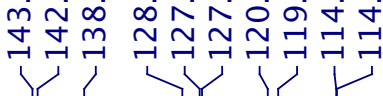

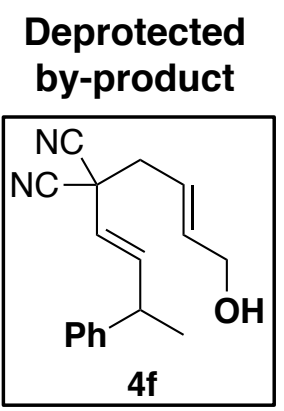

$4 f$
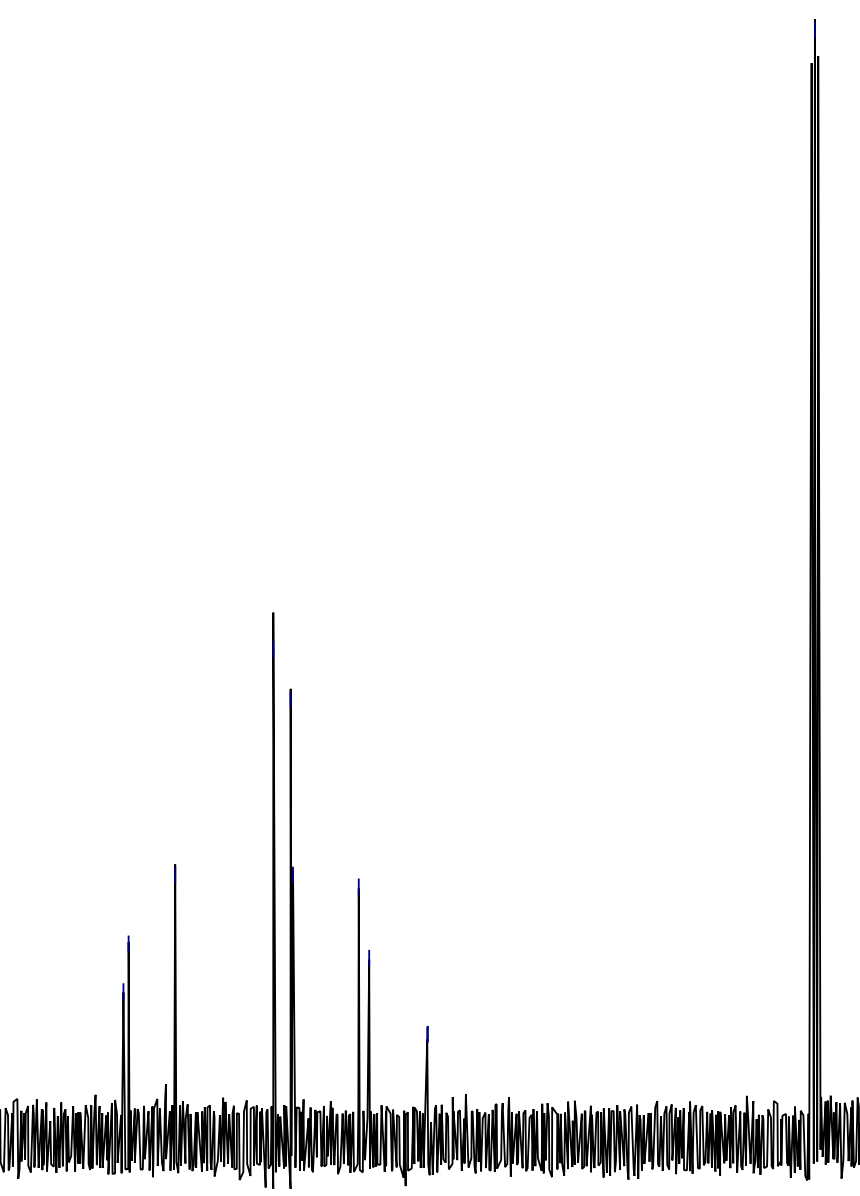
商

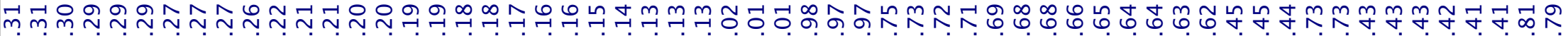

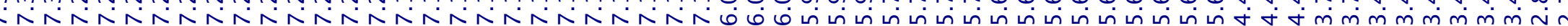

Al IIII)
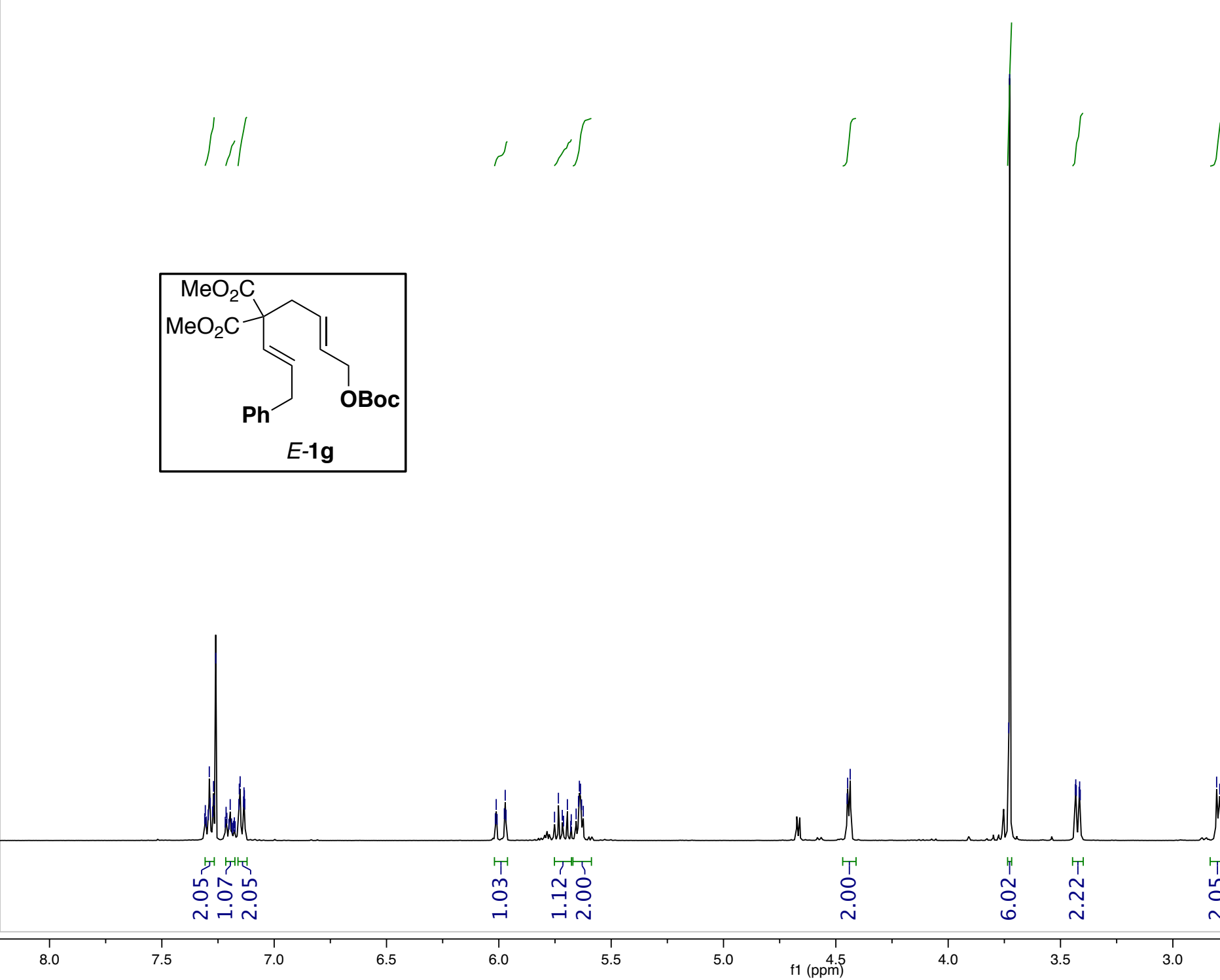

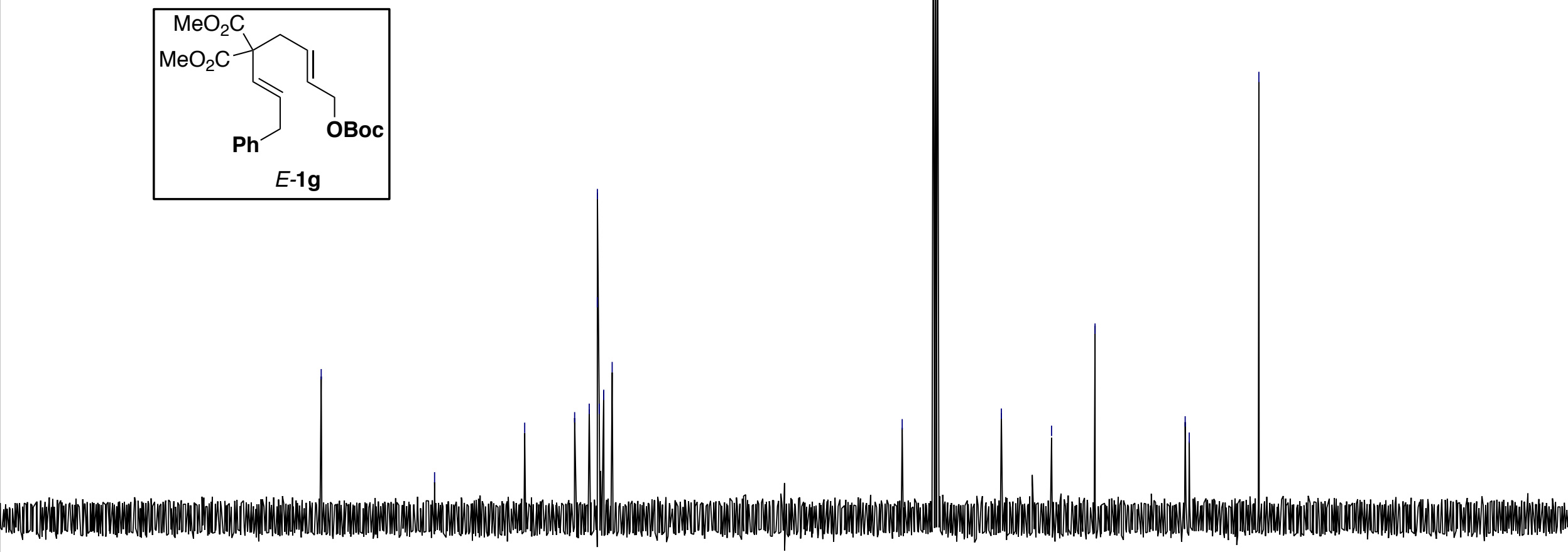

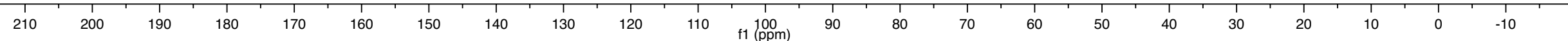


$\frac{m}{U}$
0
0

11

$\int / / J$

Deprotected

by-product
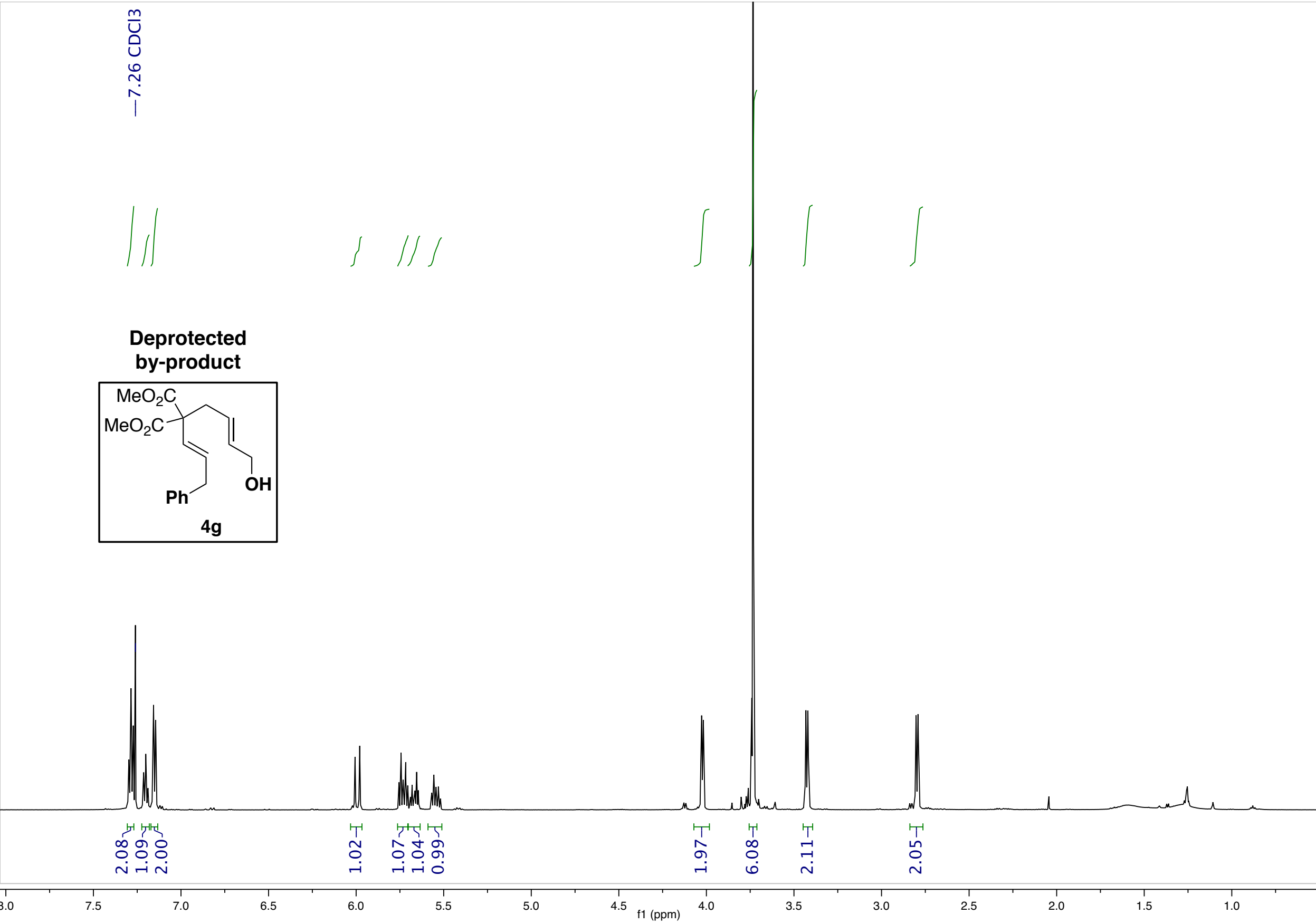


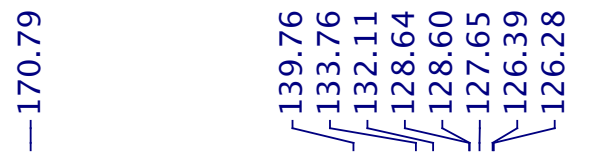

\section{Deprotected}

by-product

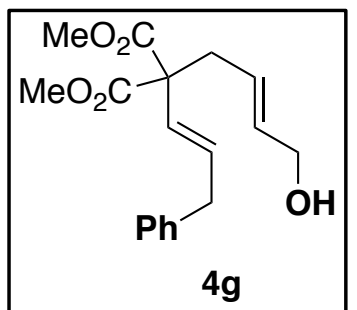

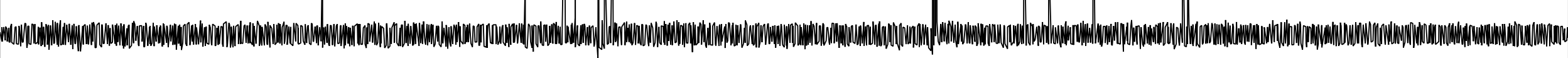

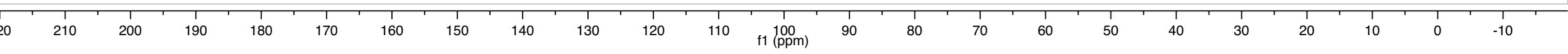


产

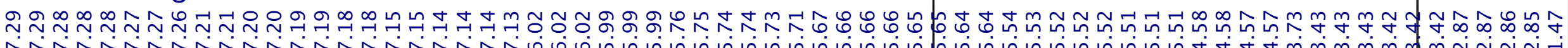

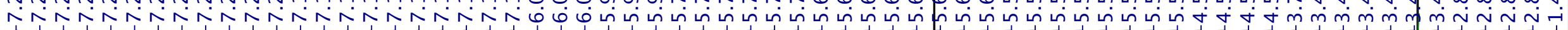

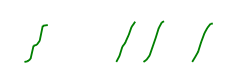

바맏

กั๊

Ni i

1

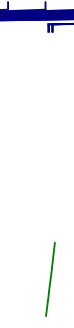

III In
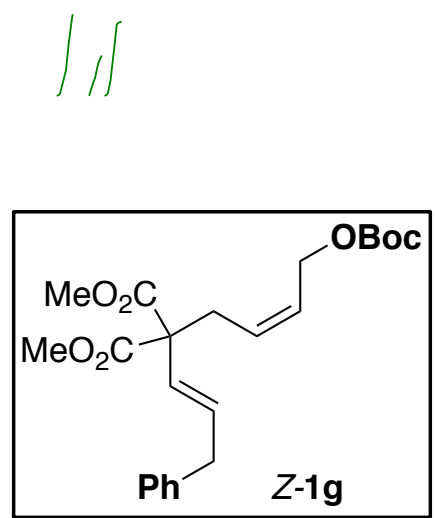
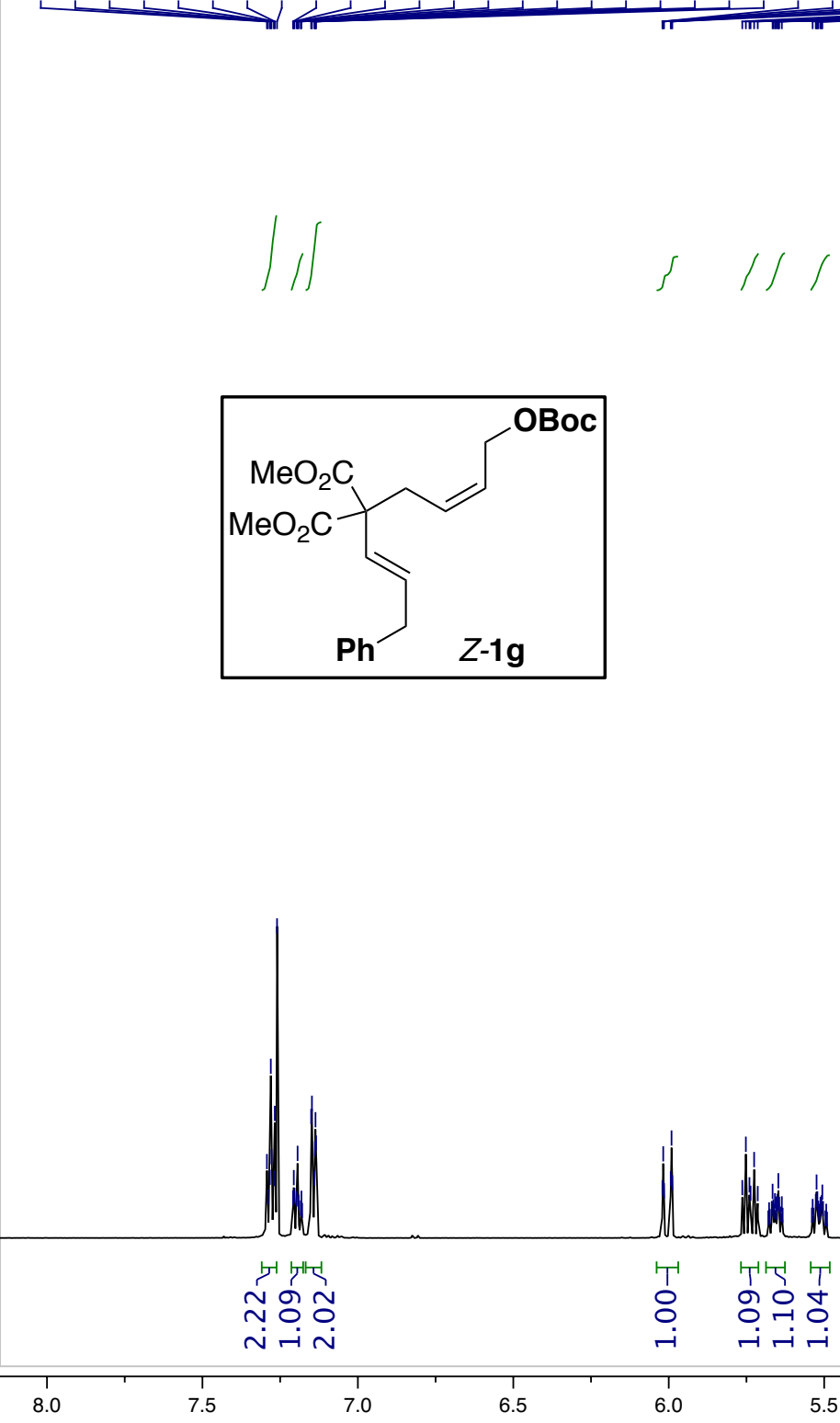

8.0 


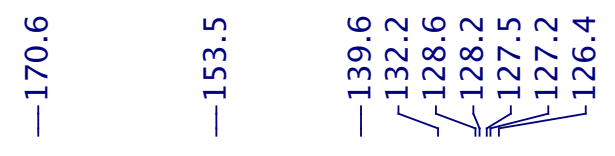
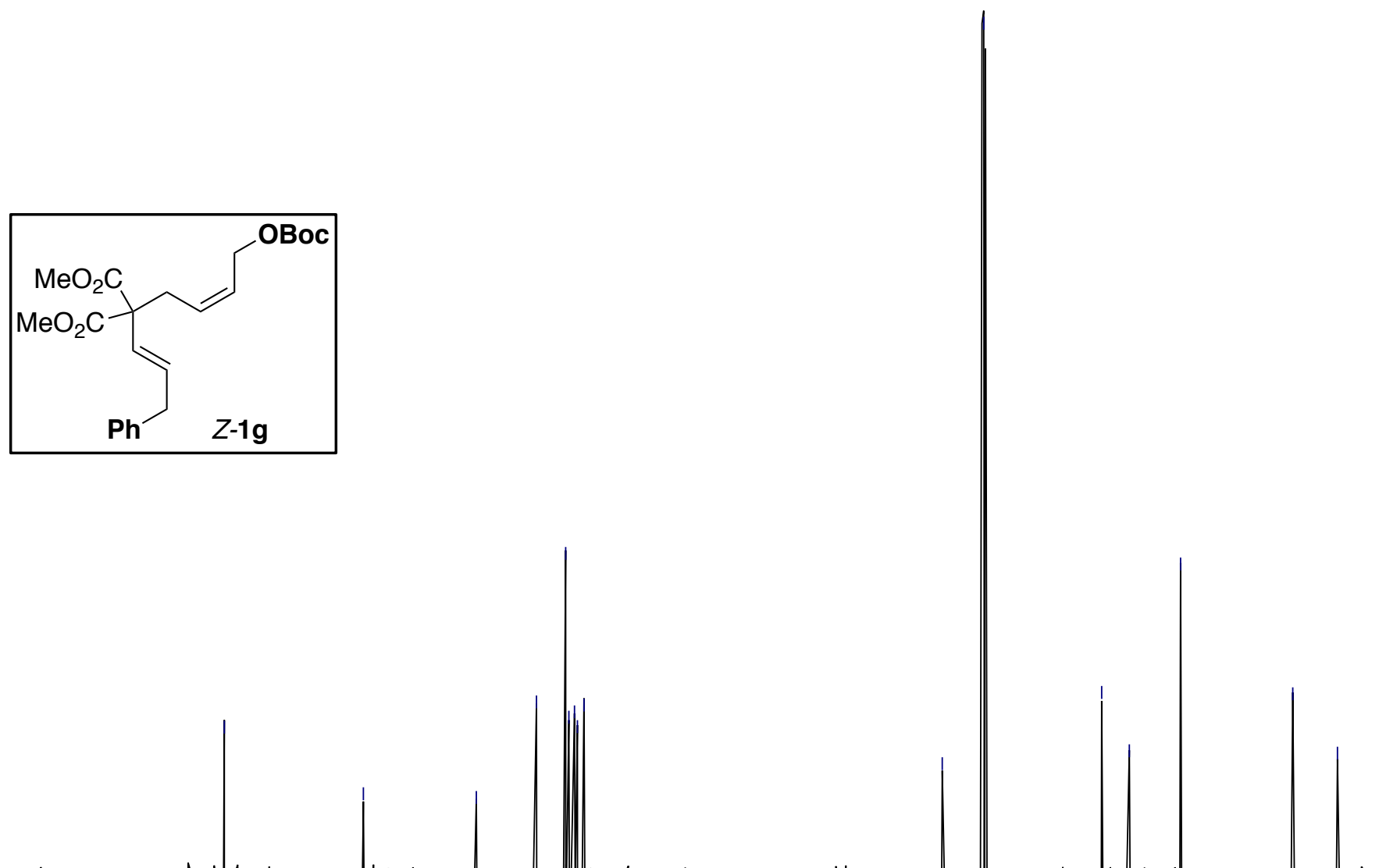

Co

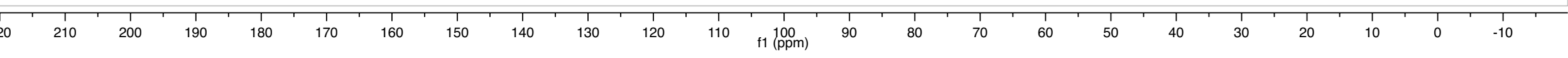


$\int /$

$\iint \Gamma$

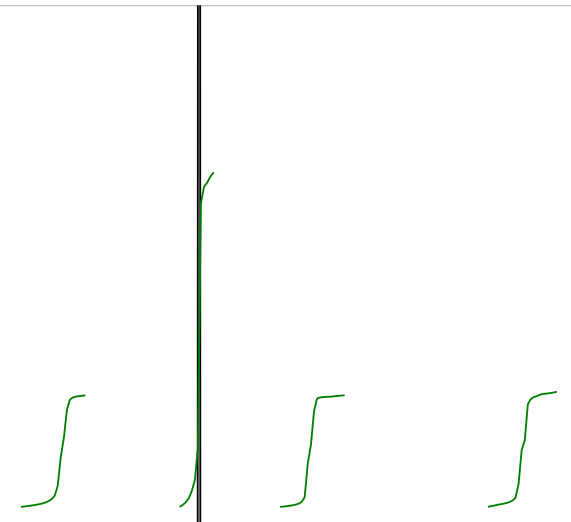

Deprotected by-product
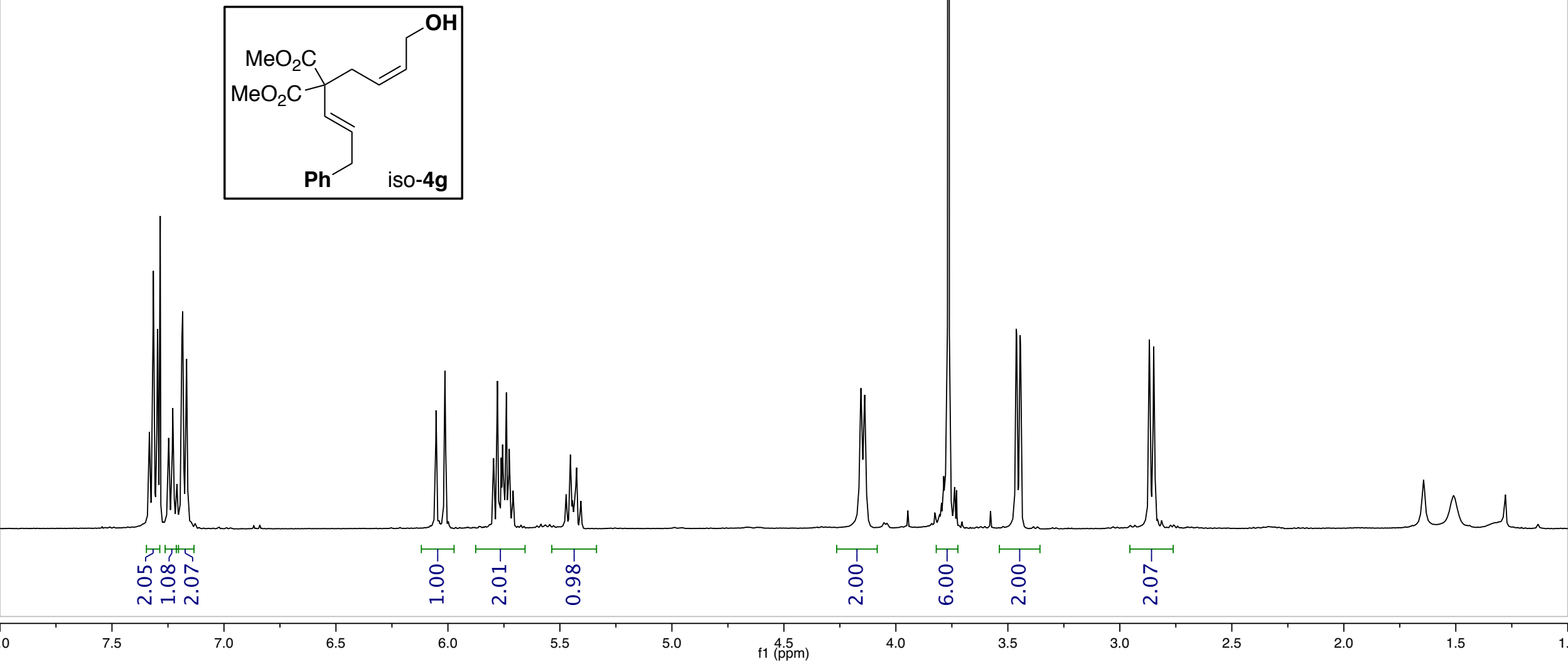

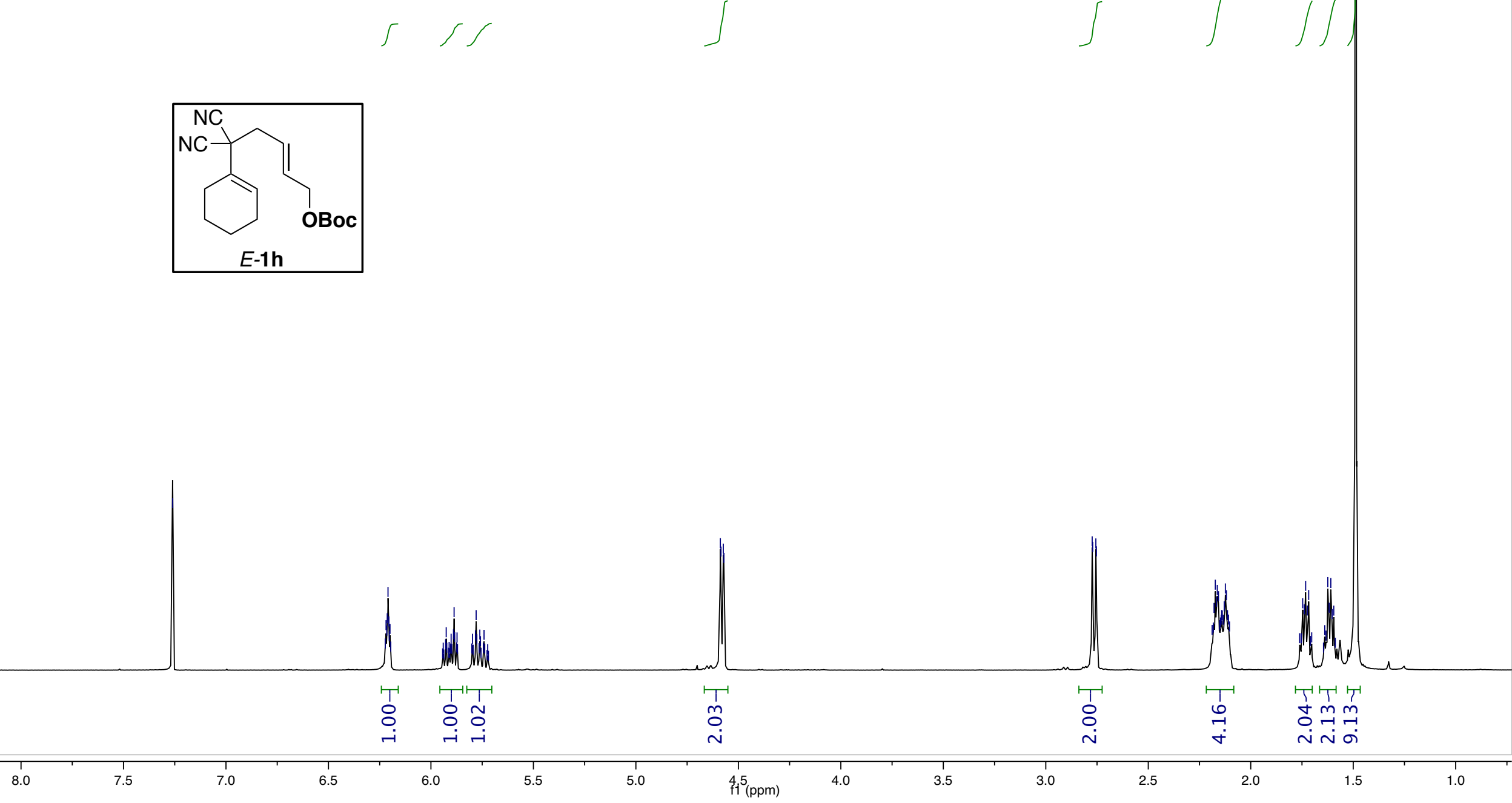


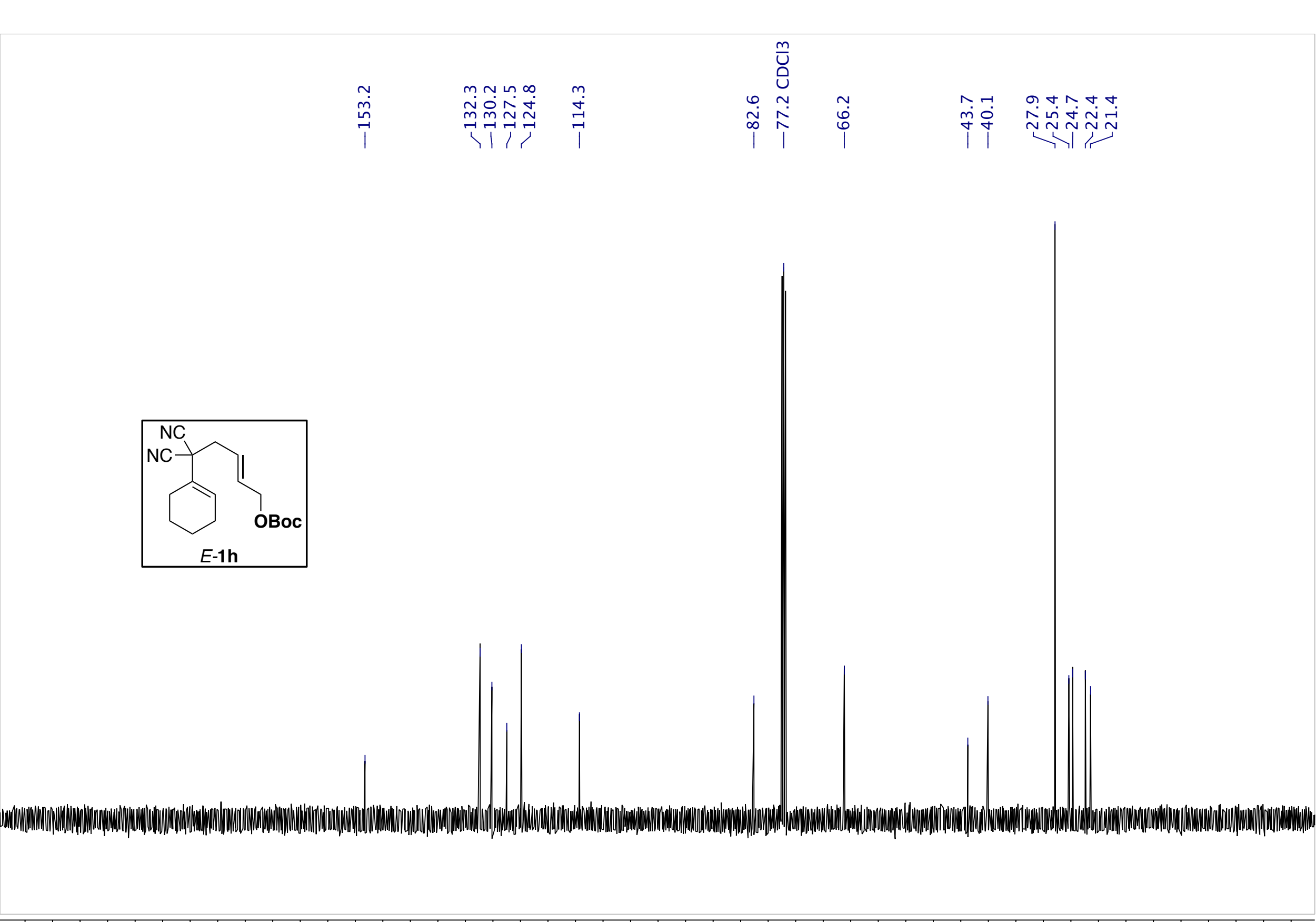




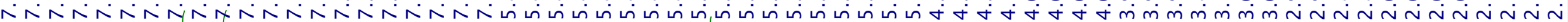
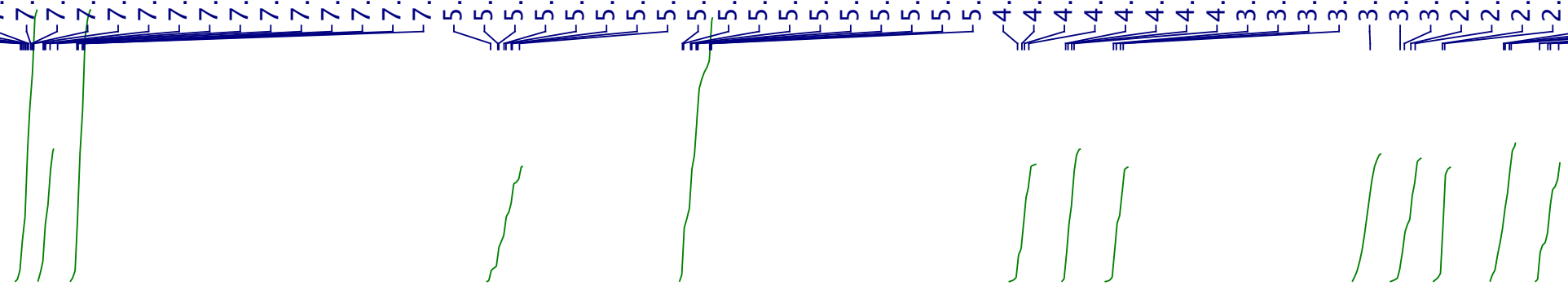

\section{5:1 d.r.}
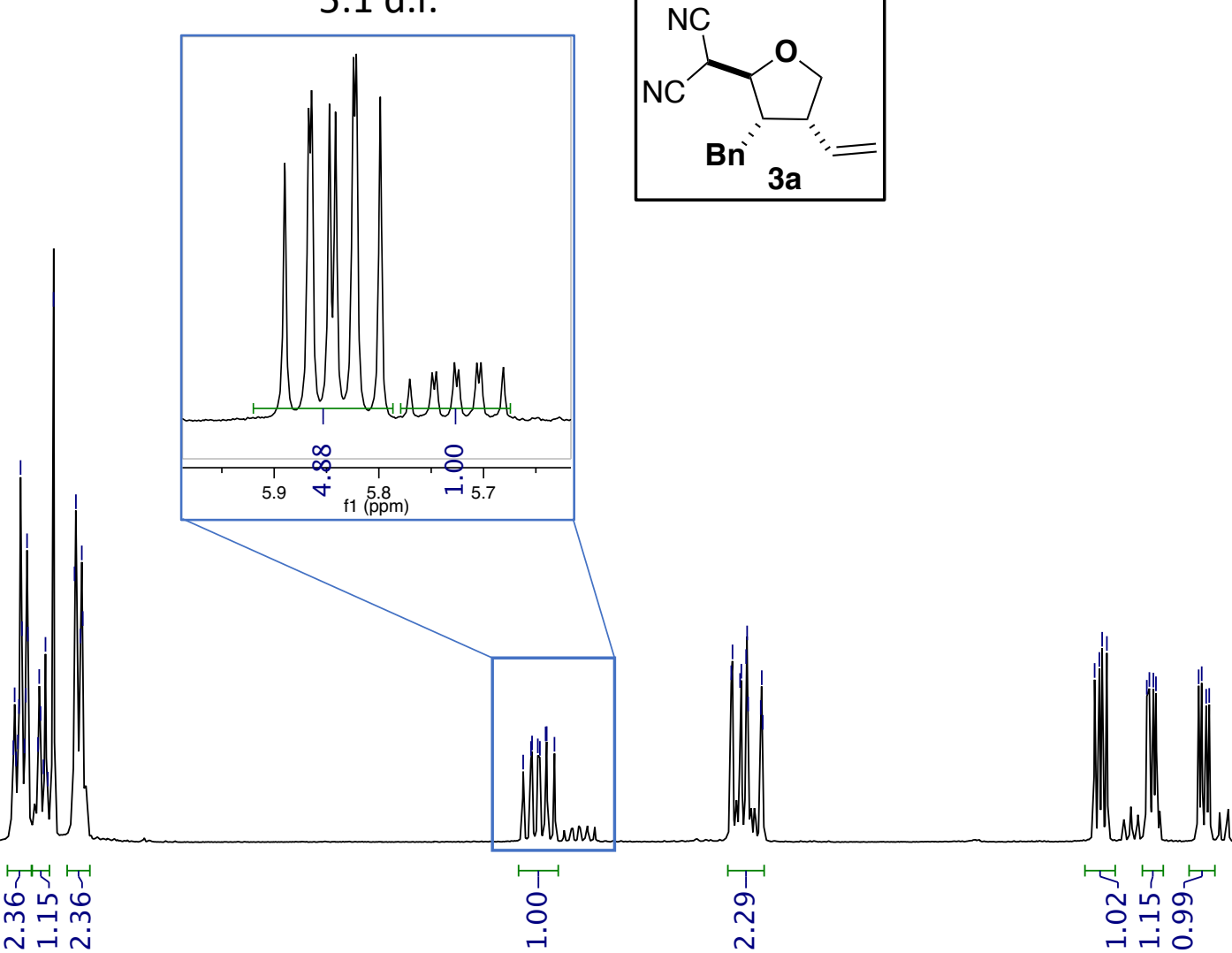

T1

पi

ชิ กิ

8

ำ

-i 


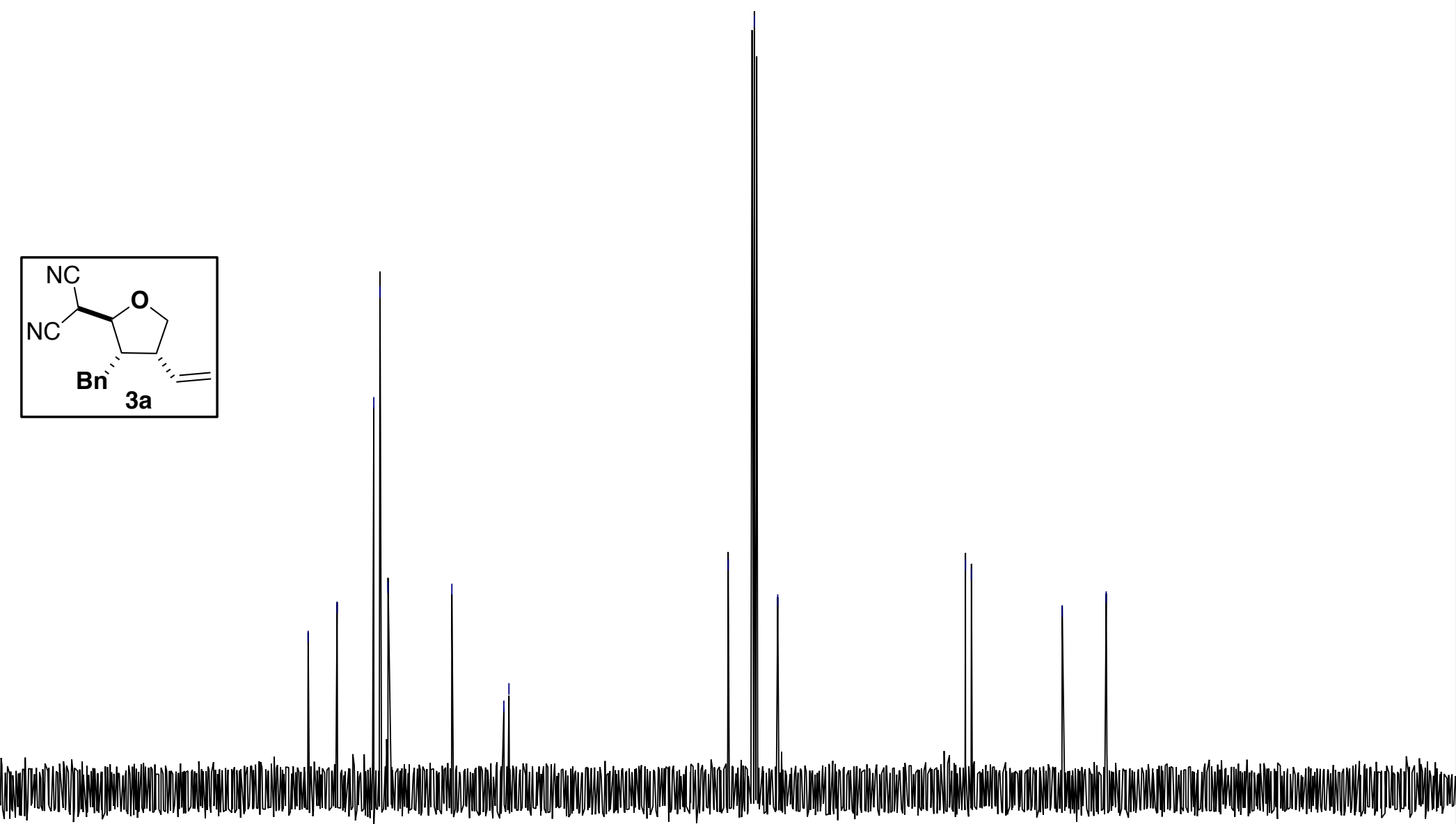




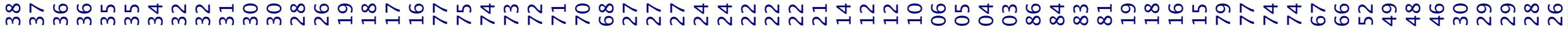

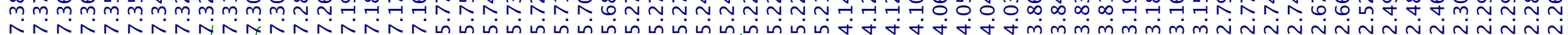
(

8:1 d.r.
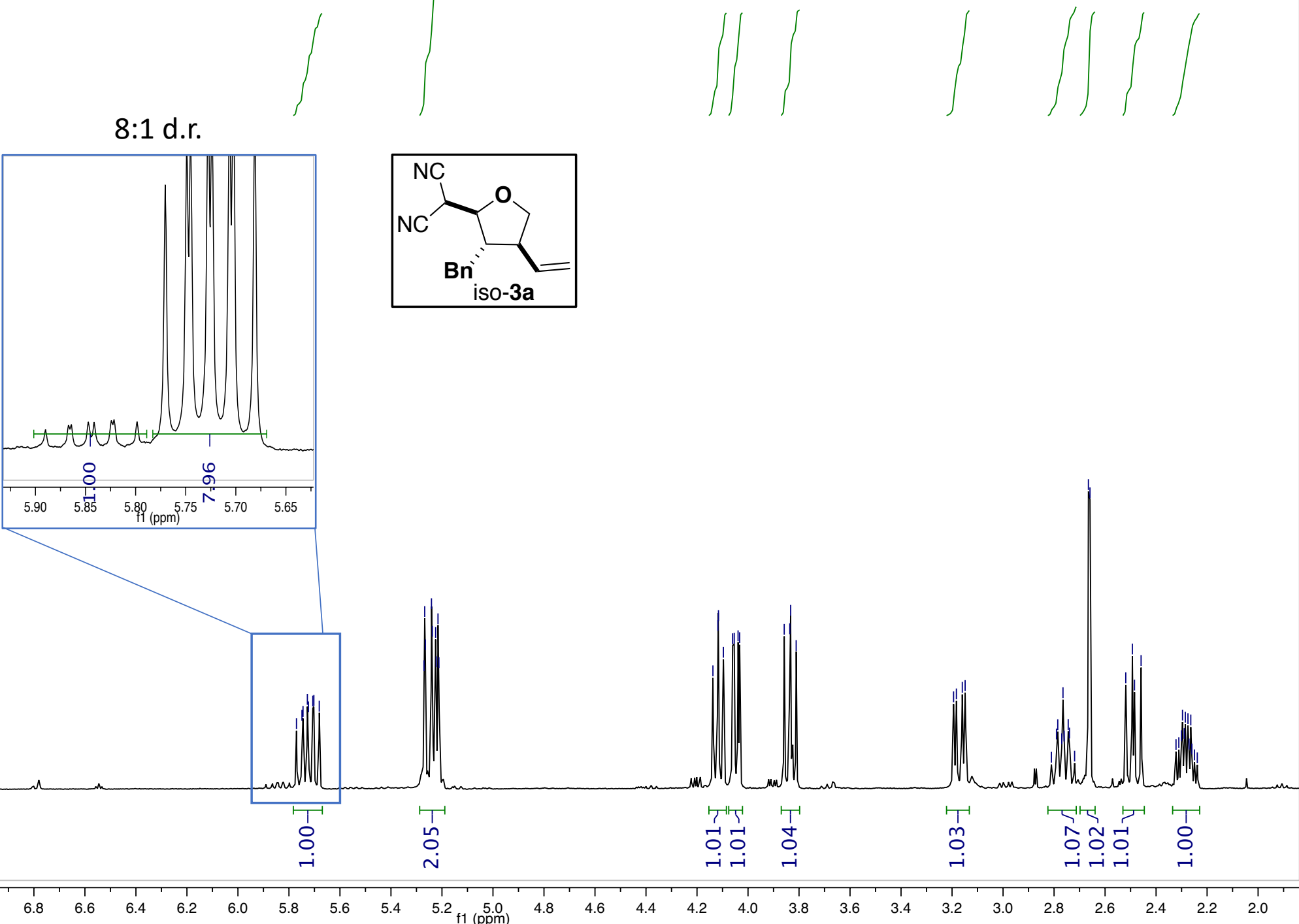

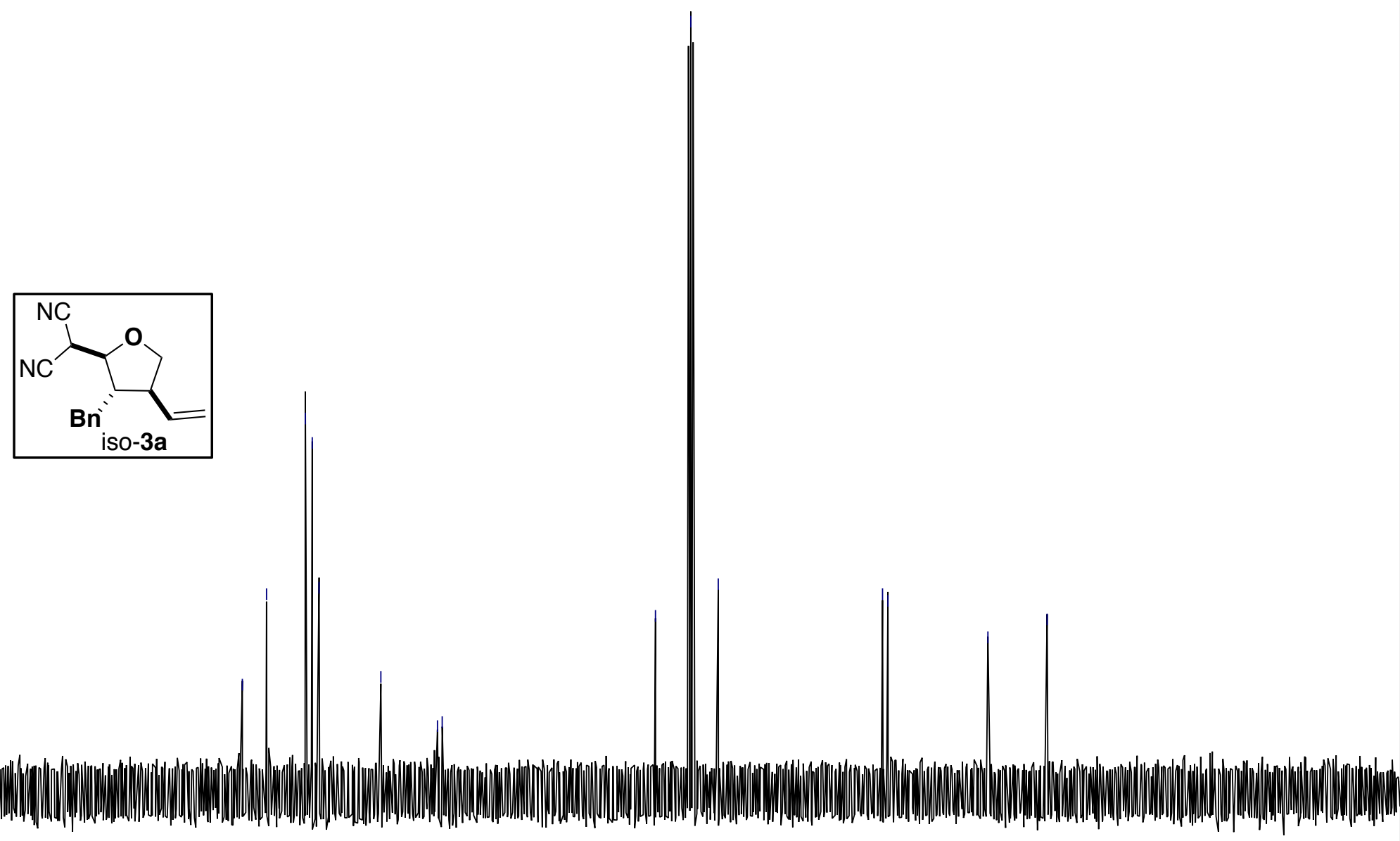


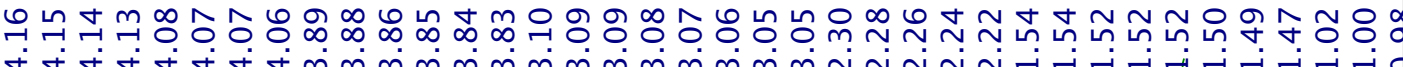
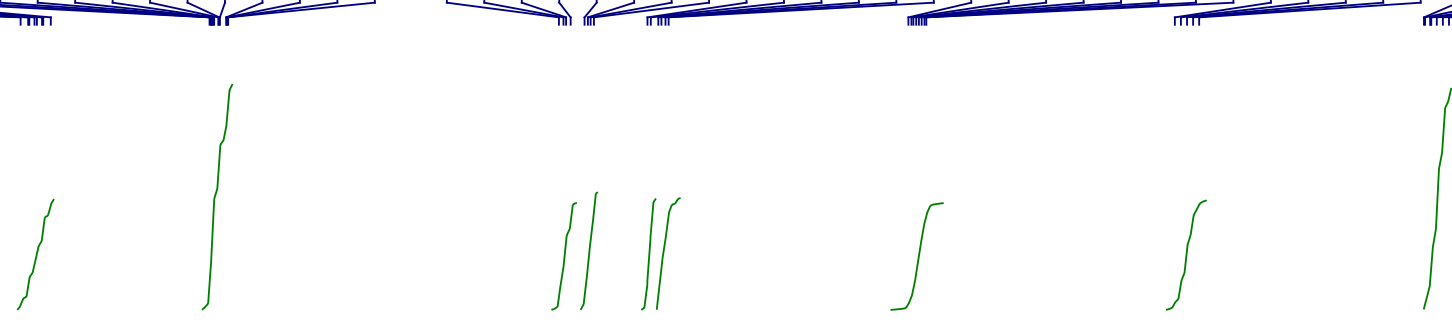

$8: 1$ d.r
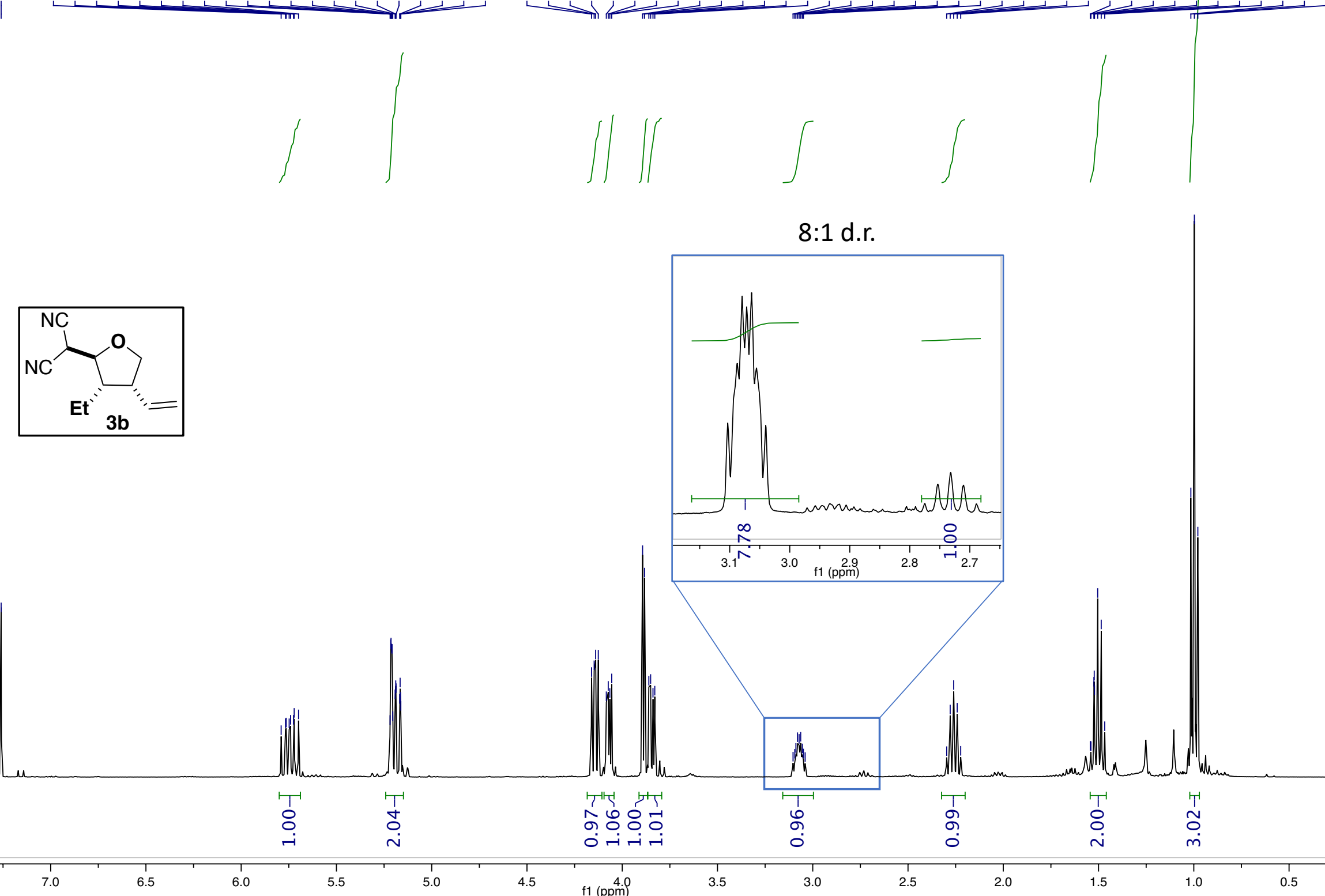


\begin{tabular}{|c|c|c|c|c|c|c|}
\hline & mo 0. & 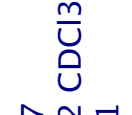 & & & & \\
\hline & 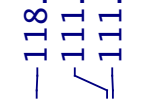 & în & $\begin{array}{ll}\infty & m \\
0 & 0 \\
0 & 0 \\
1 & 0\end{array}$ & $\stackrel{\infty}{\infty}$ & $\stackrel{\vec{i}}{i}$ & $\underset{\sim}{\stackrel{\sim}{\sim}}$ \\
\hline
\end{tabular}

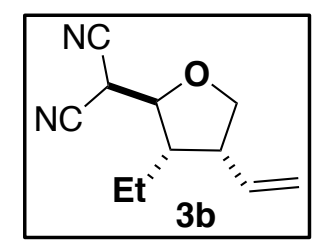

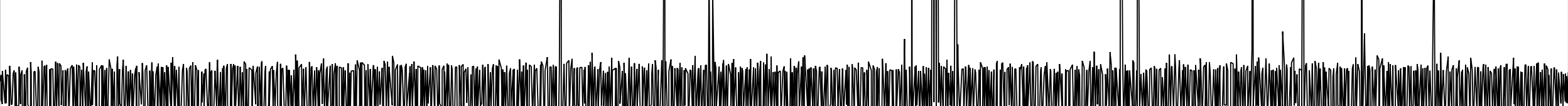

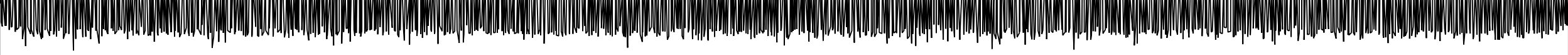

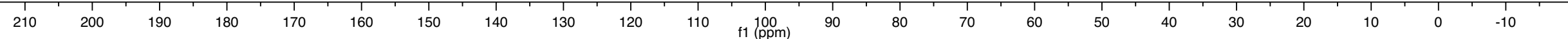



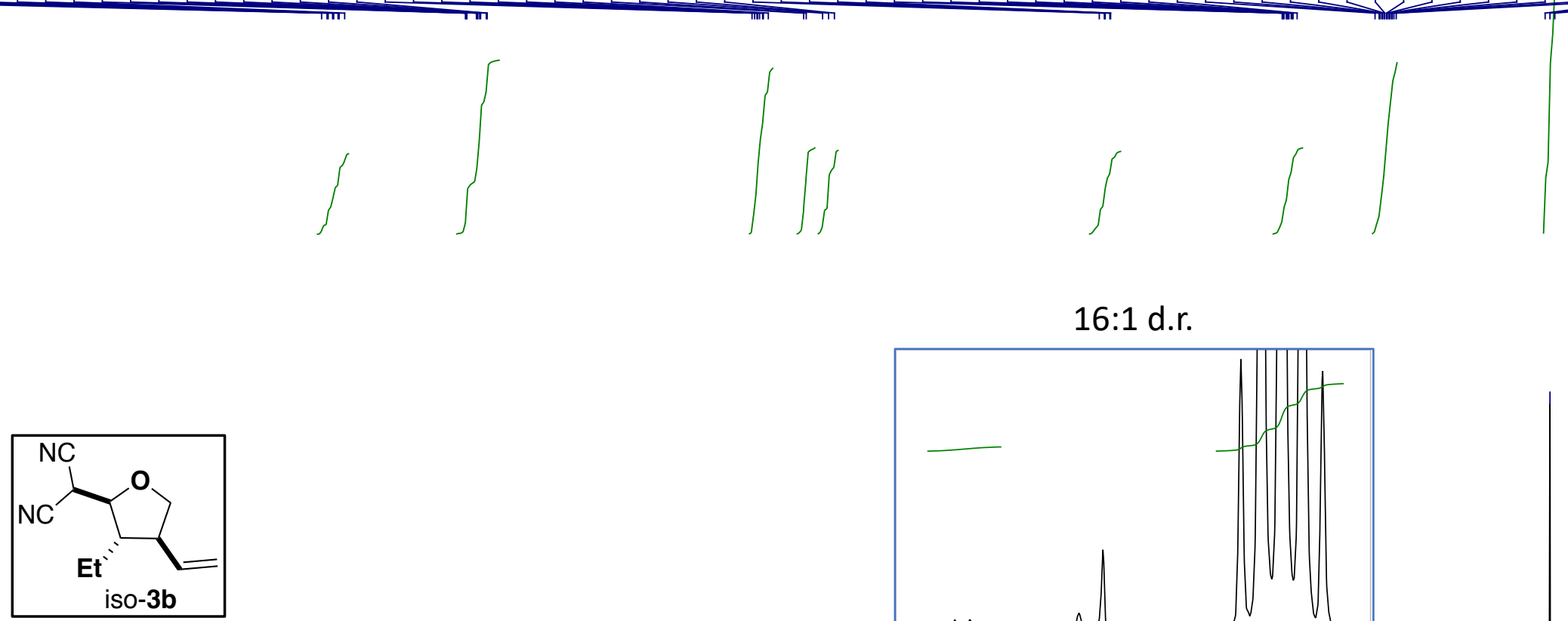

$16: 1$ d.r.
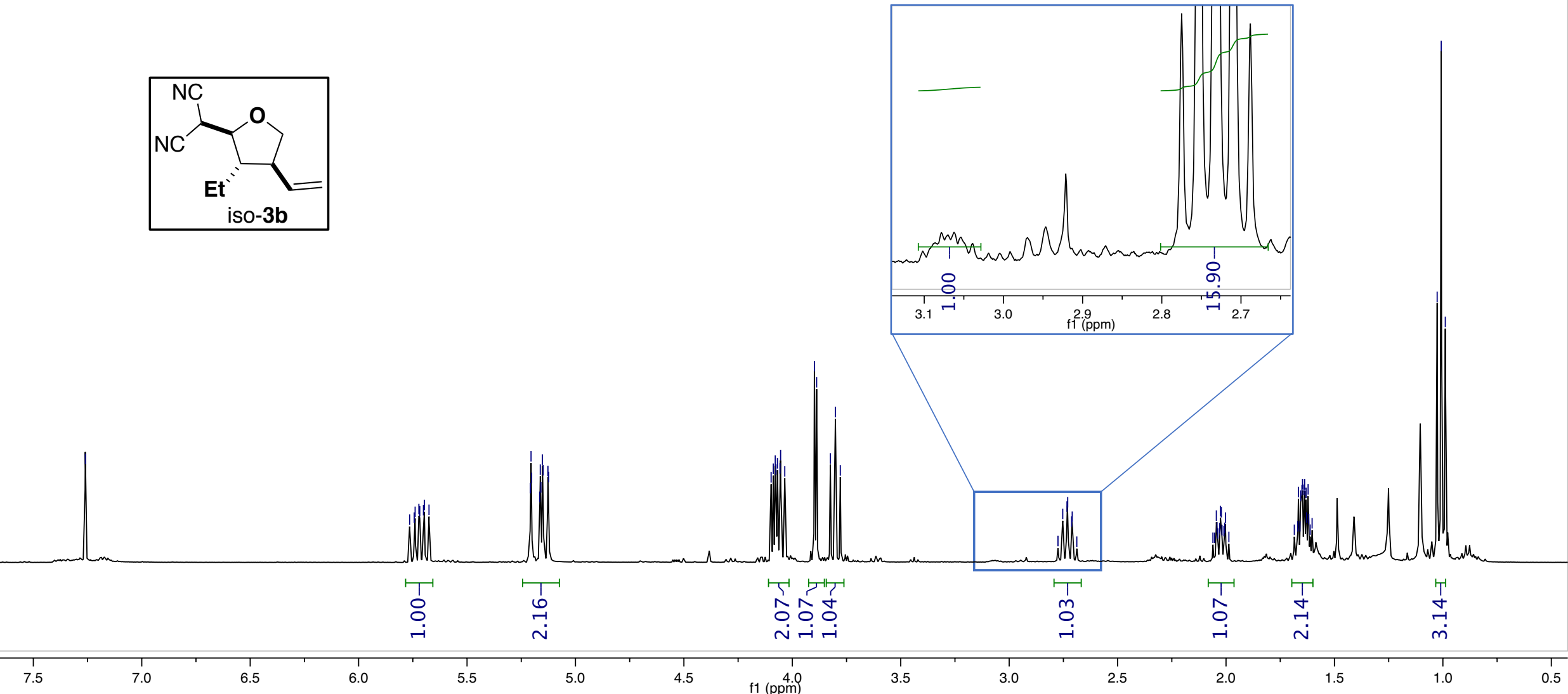




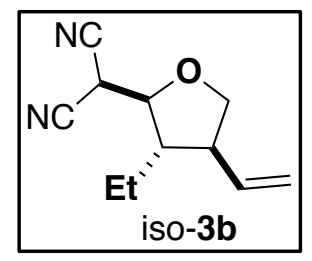

$210 \quad 200$

190
190

180

170

160

150

140

130

120

100
$\mathrm{f} 1(\mathrm{ppm})$

90

80

70

60

50

40

30

20 


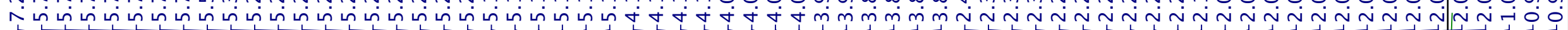
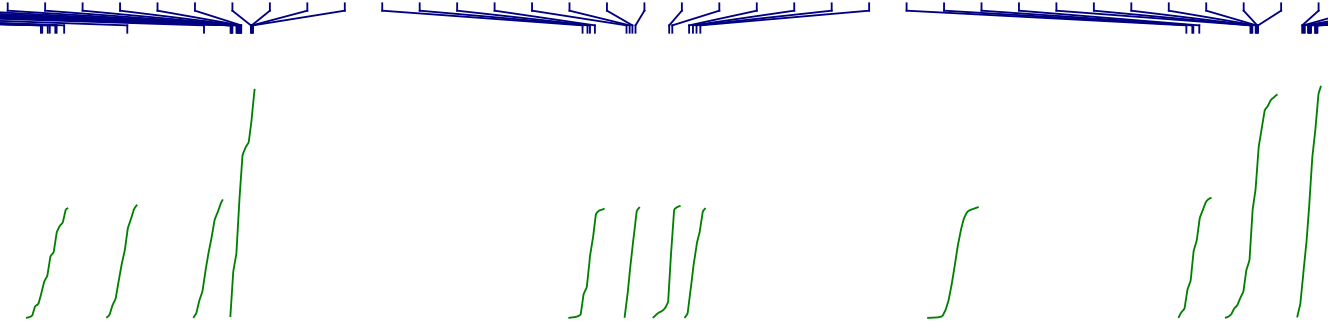

7:1 d.r.
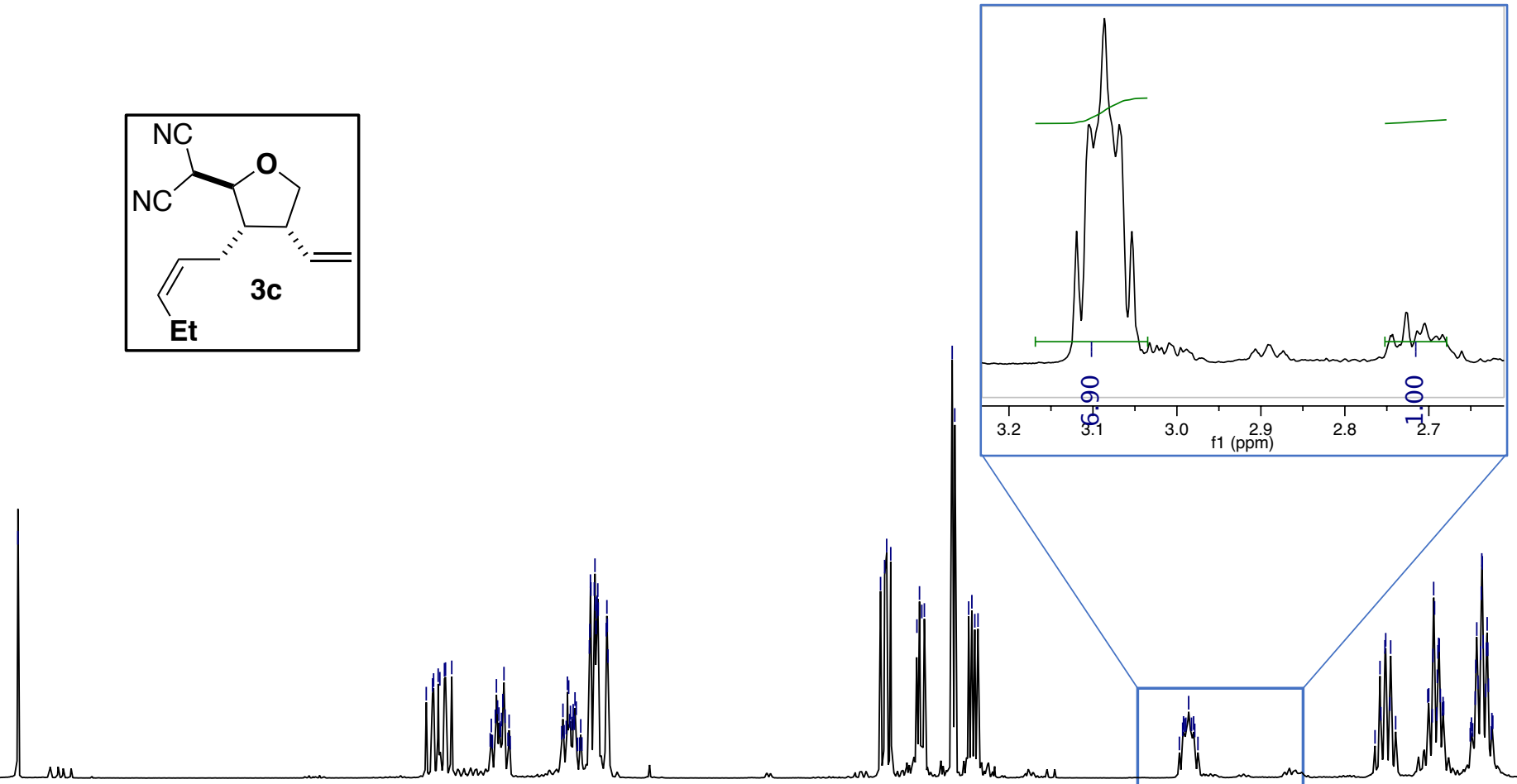

$\begin{array}{lll}\longmapsto & T & T \\ 0 & m & \infty \\ 0 & 0 & 0 \\ -i & -i & r i\end{array}$

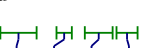

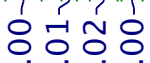

다다당
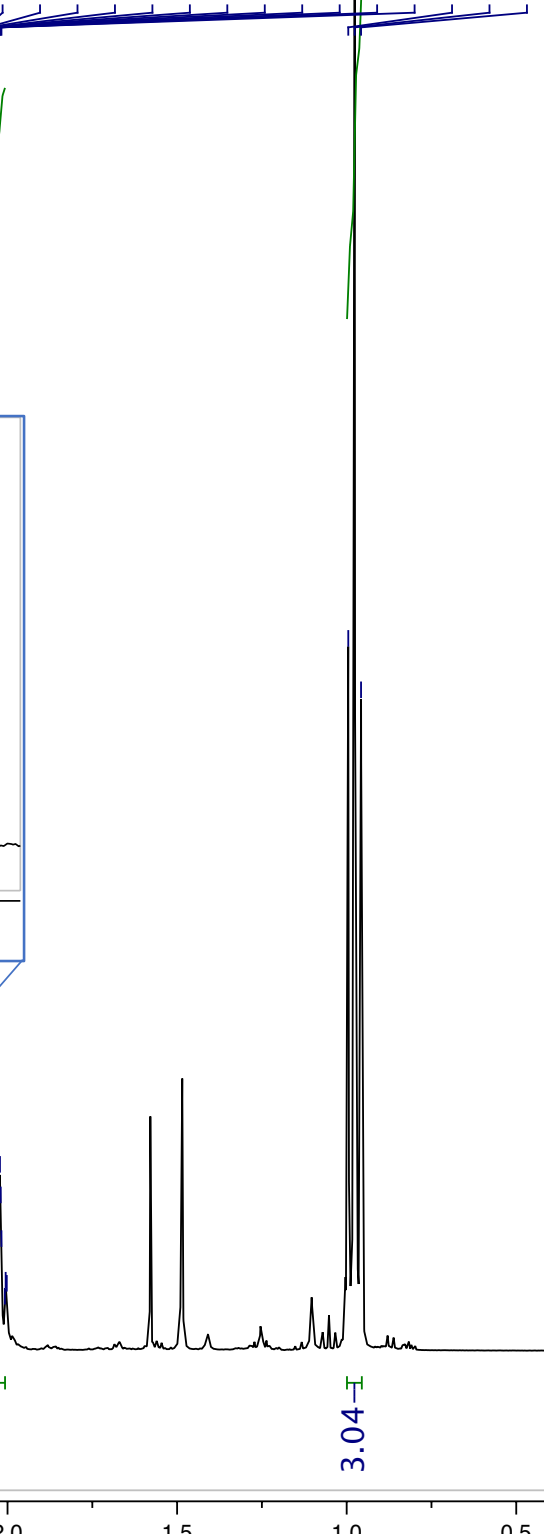


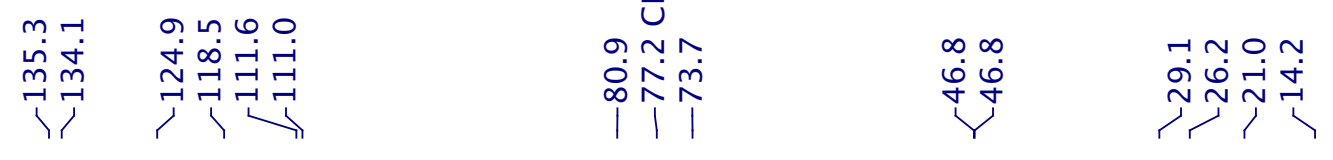

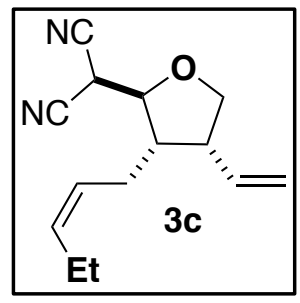

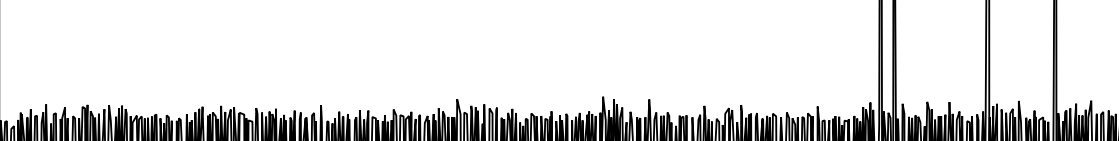

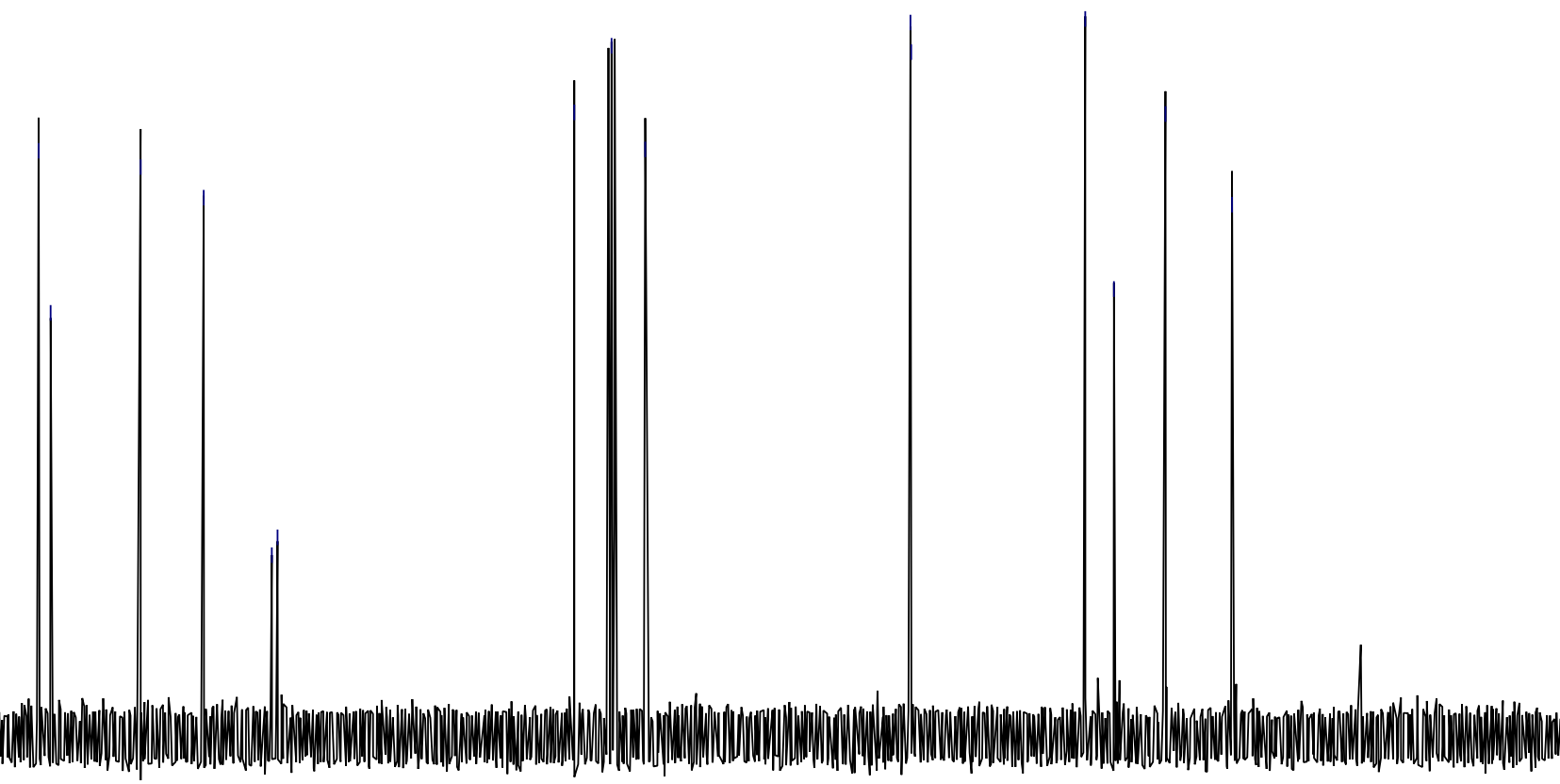



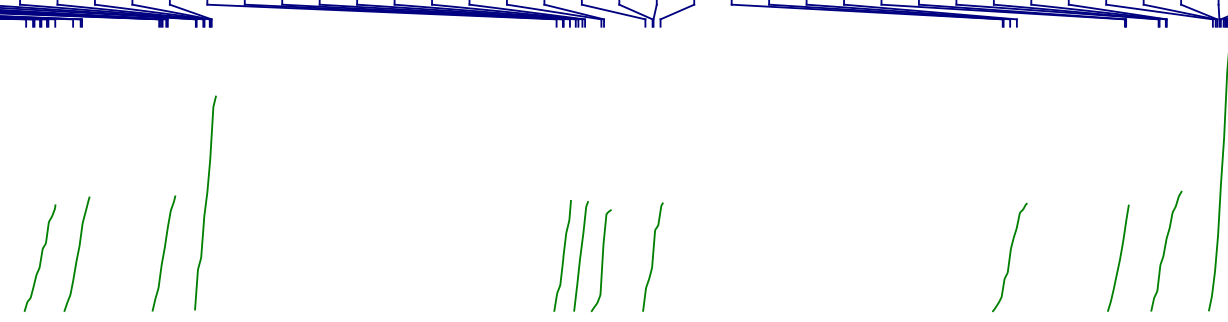

10:1 d.r.
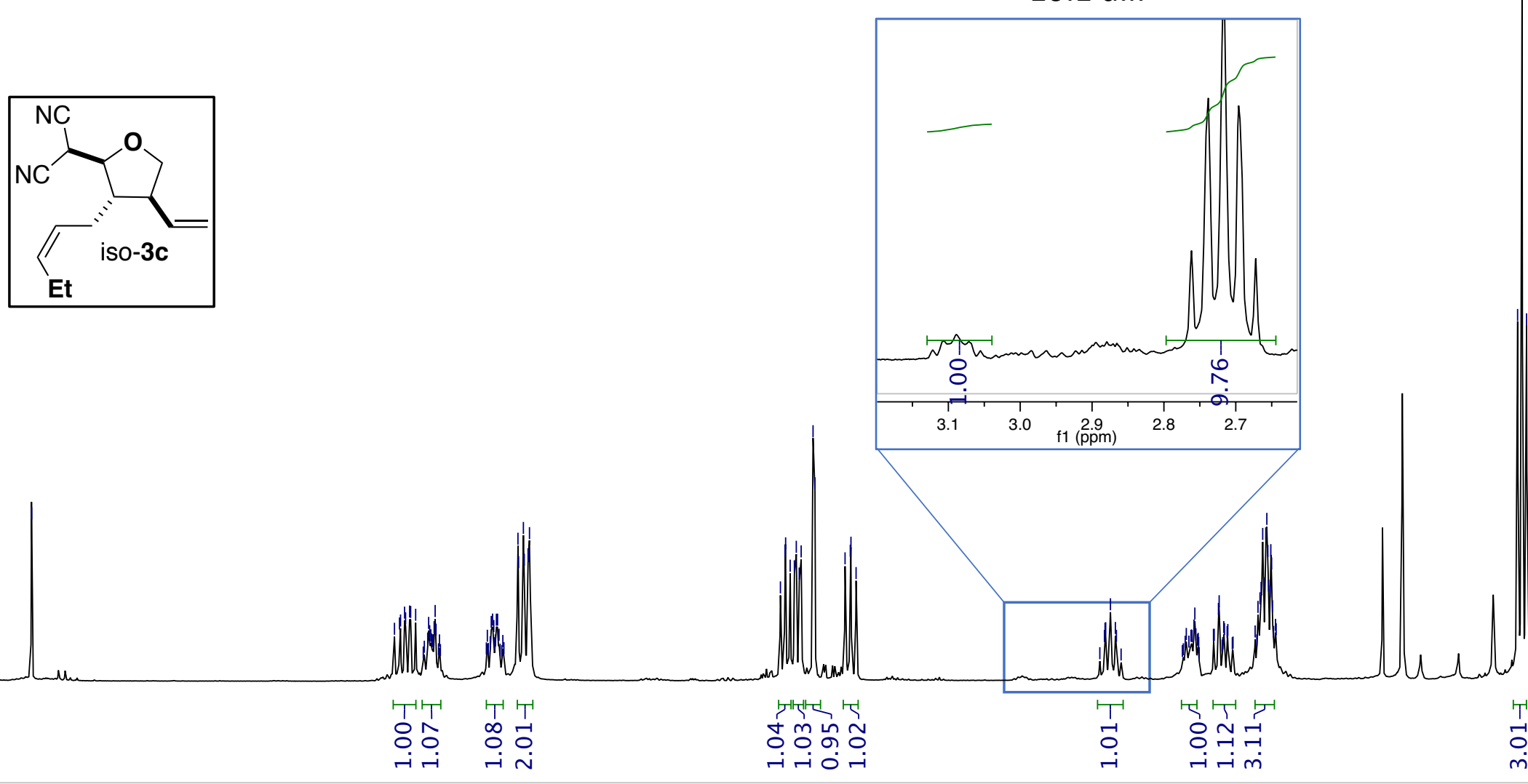
ț $\operatorname{m} \cong$

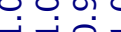

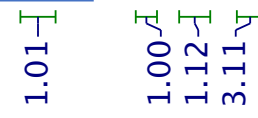

ir $-\dot{i} \dot{n}$ 


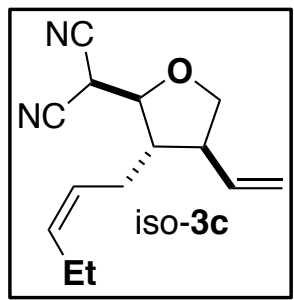




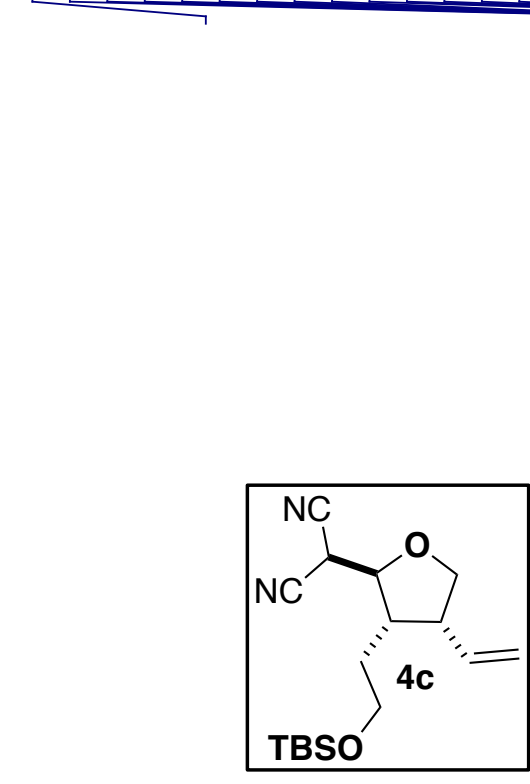

\section{4:1 d.r.}

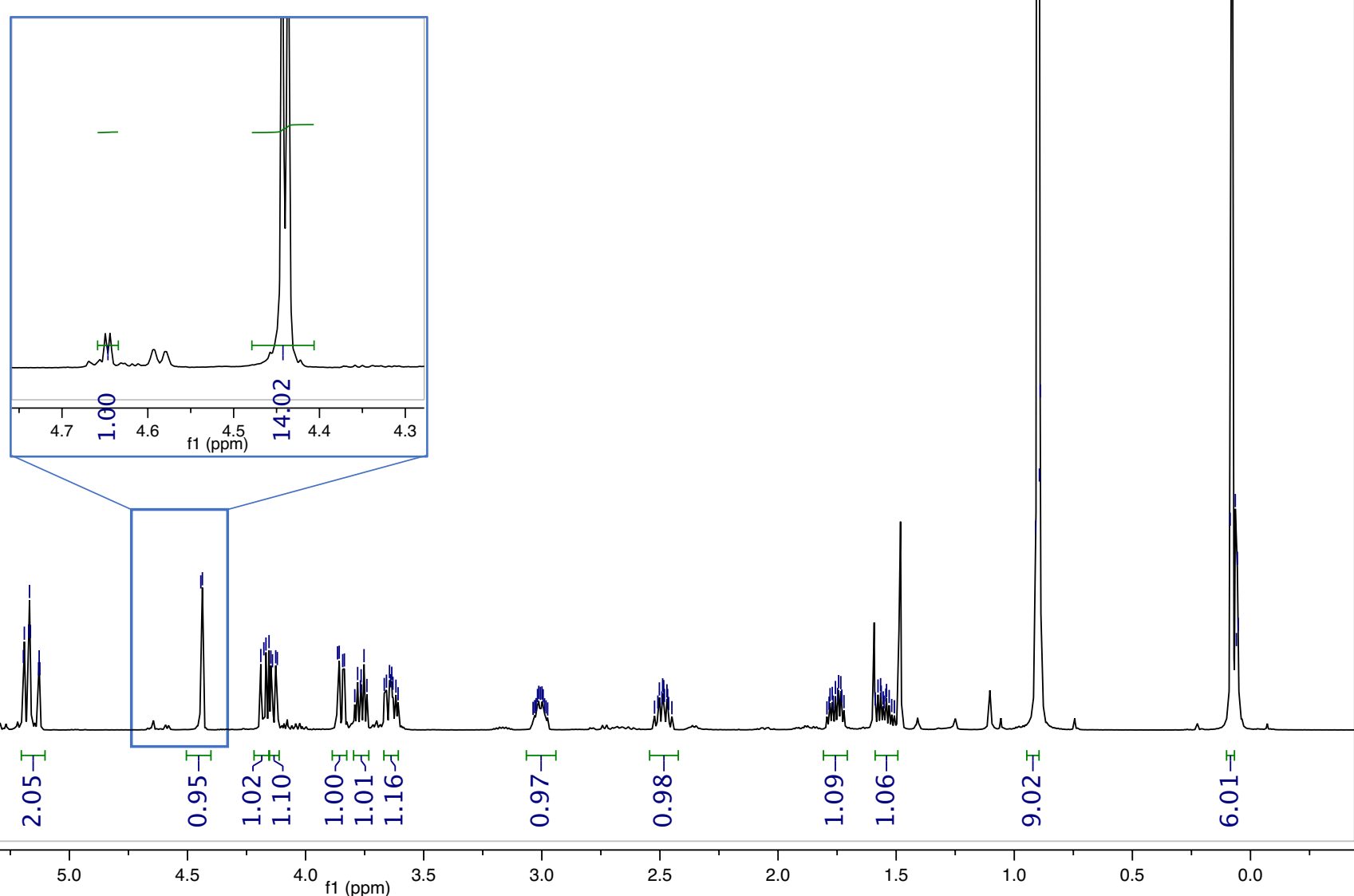






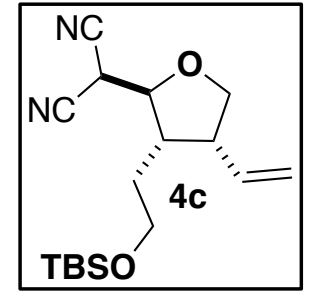

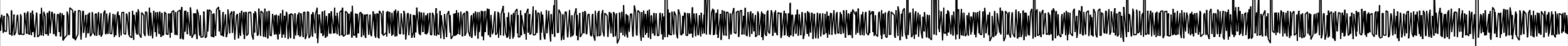




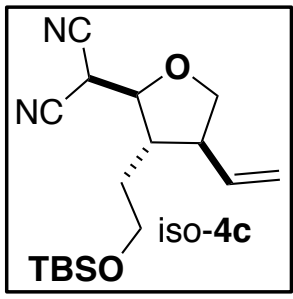




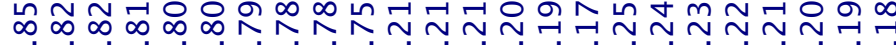

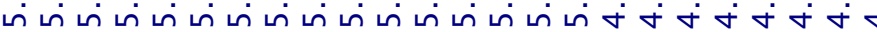

în

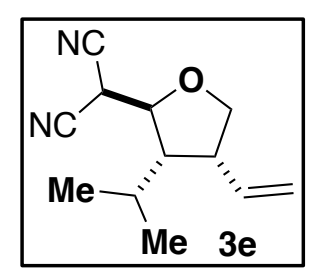

e $3 e$
我

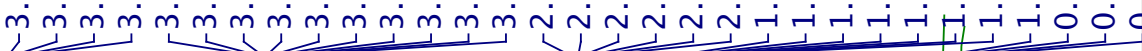

9:1 d.r.

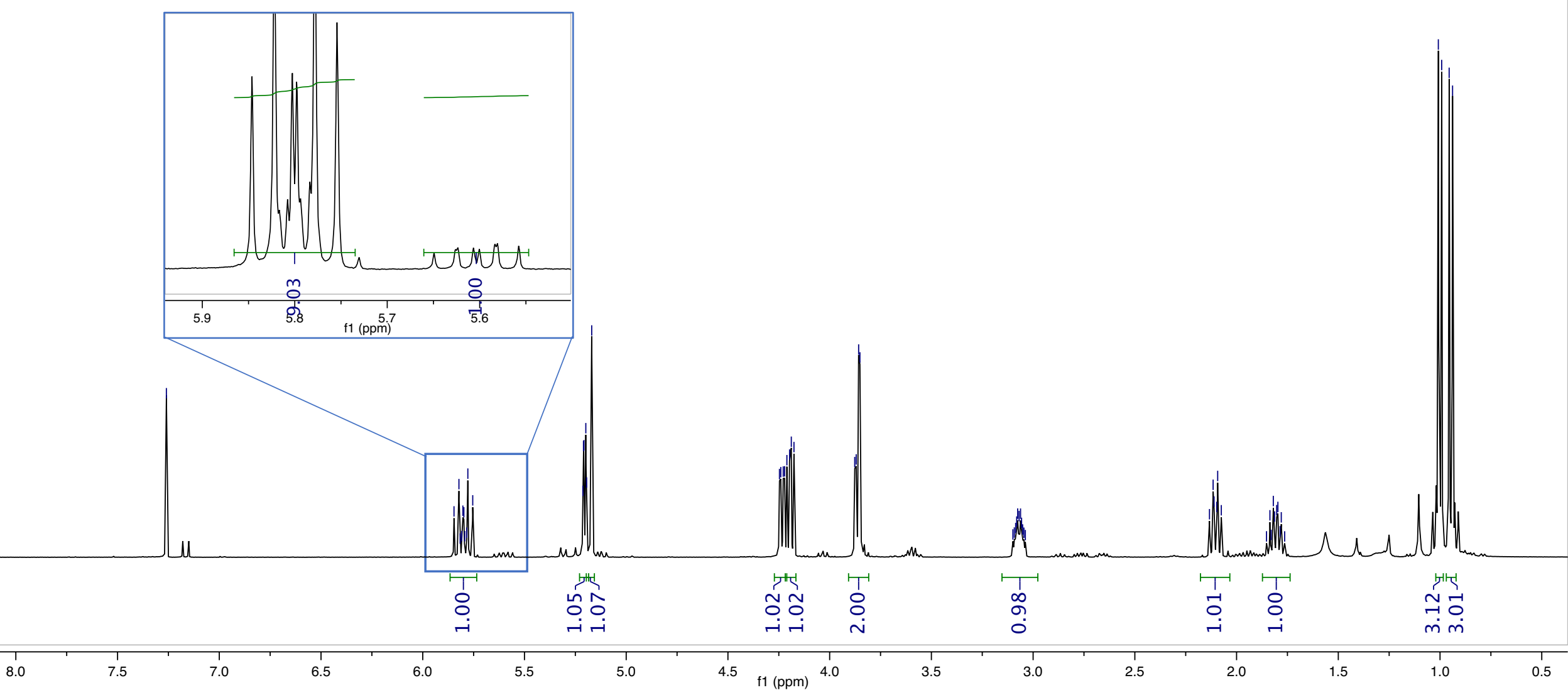

8

กิ
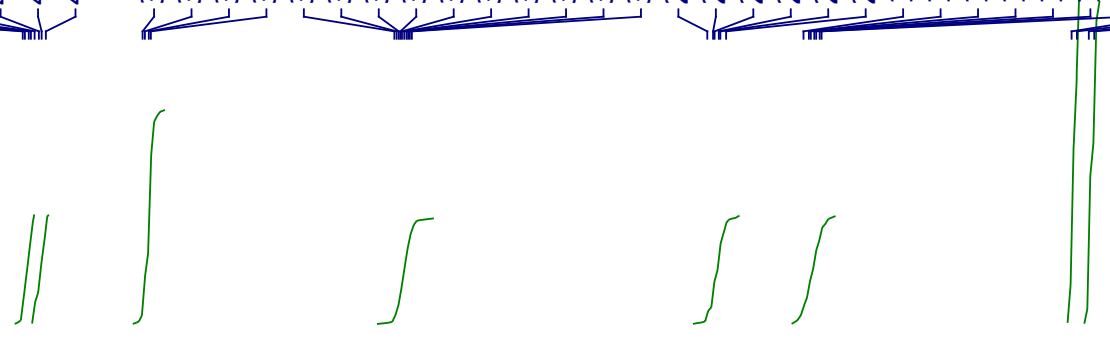


亚

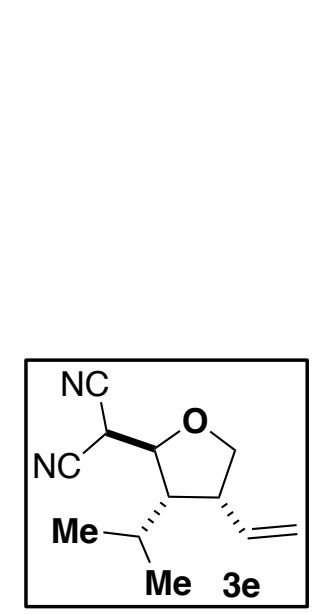

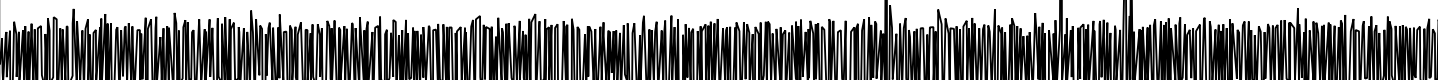
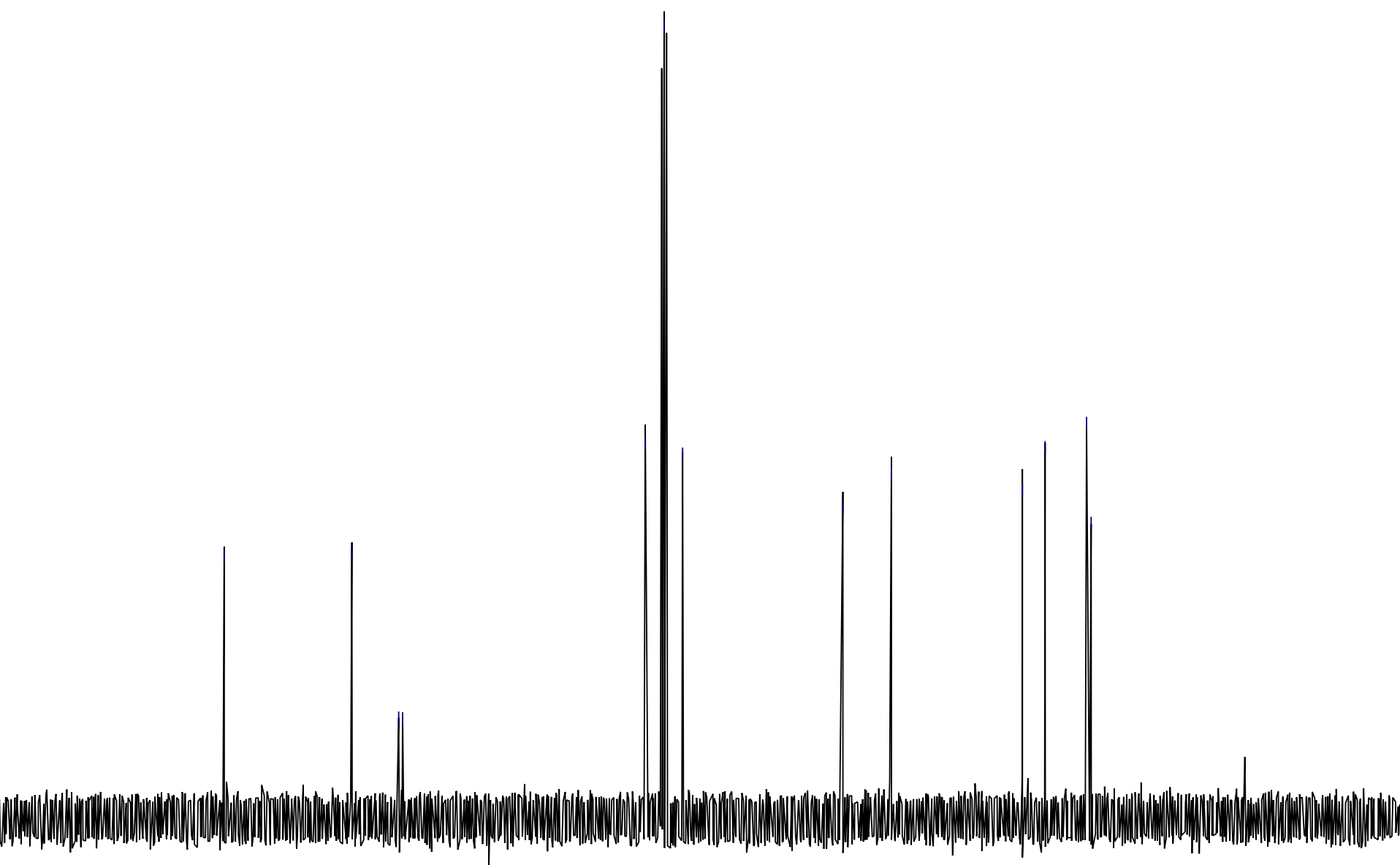
감우

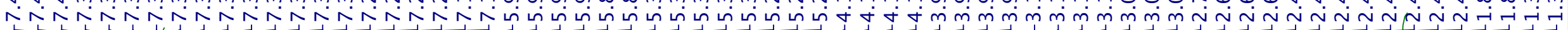
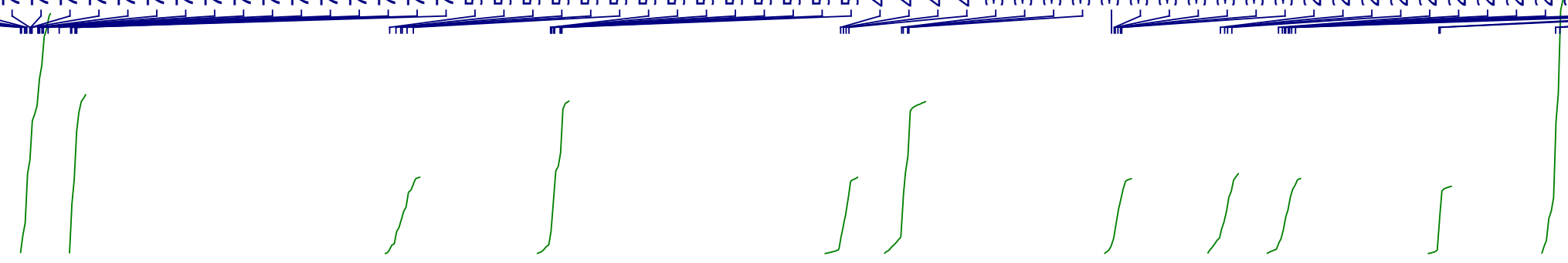

\section{Diastereomer 1}
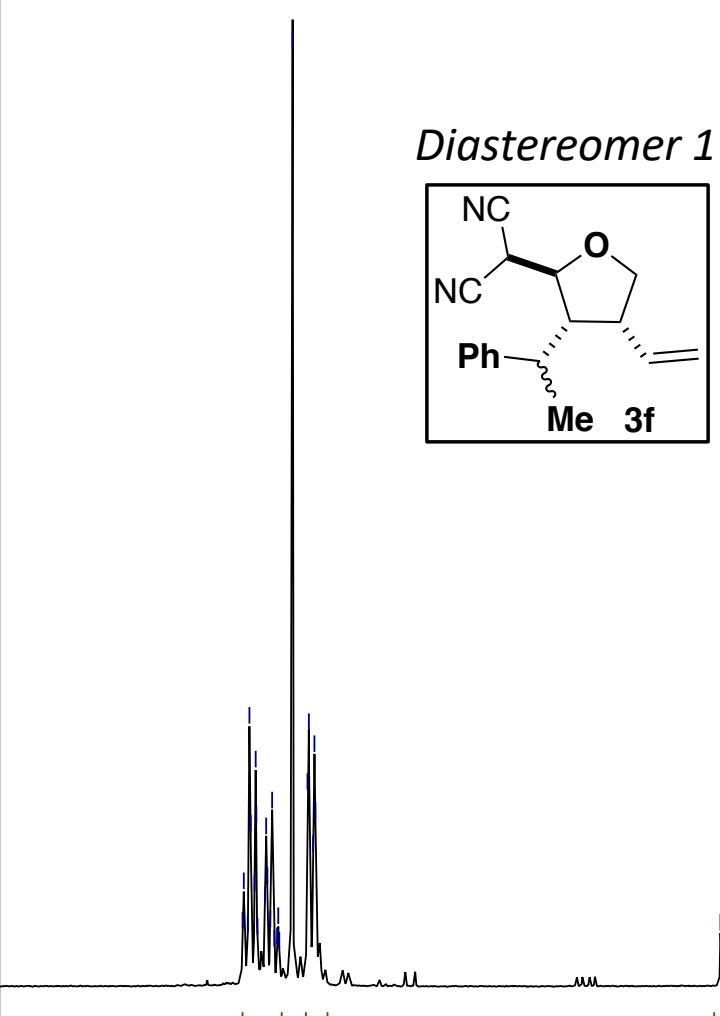

$\begin{array}{cc}\models & T \\ \dot{J} & \infty \\ \dot{\sim} & 0 \\ \dot{m} & \sim\end{array}$

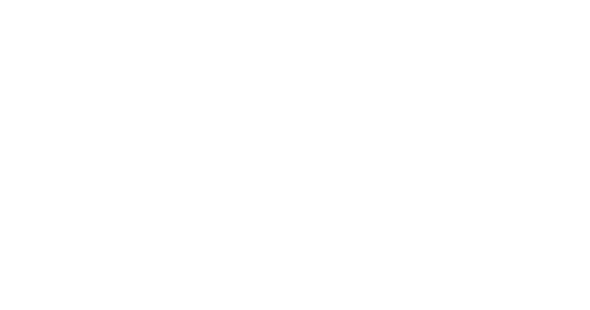




\section{Diastereomer 1}

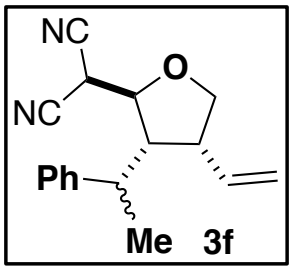



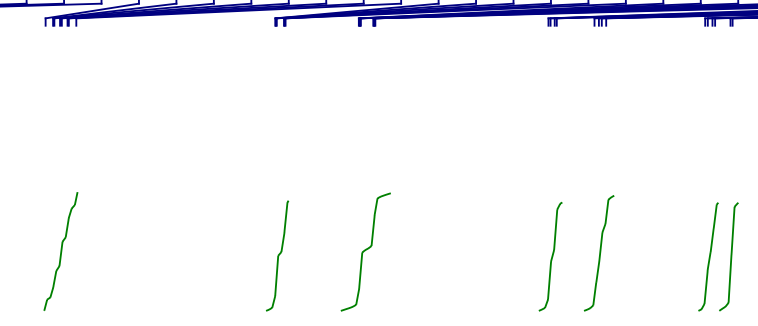

\section{Diastereomer 2}

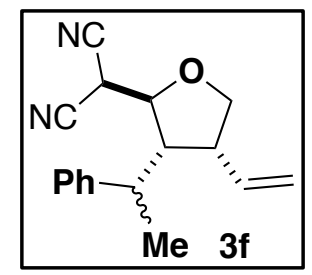

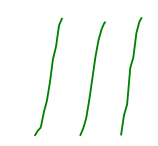




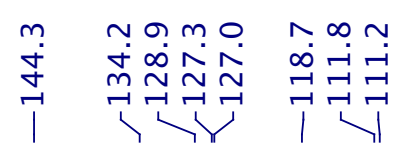

\section{Diastereomer 2}

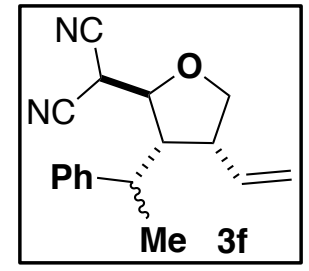

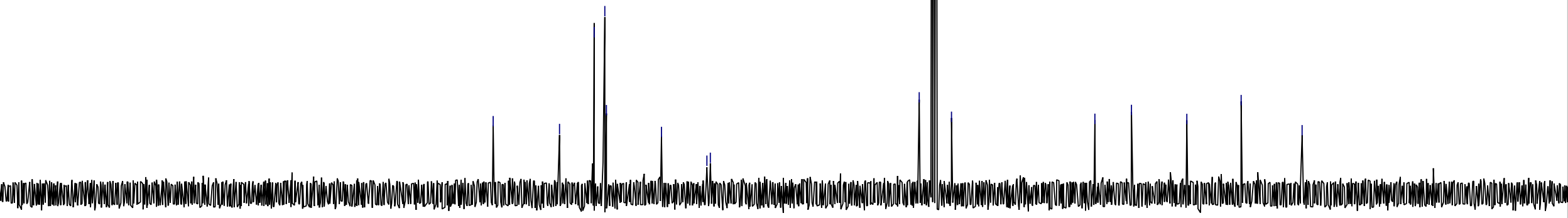




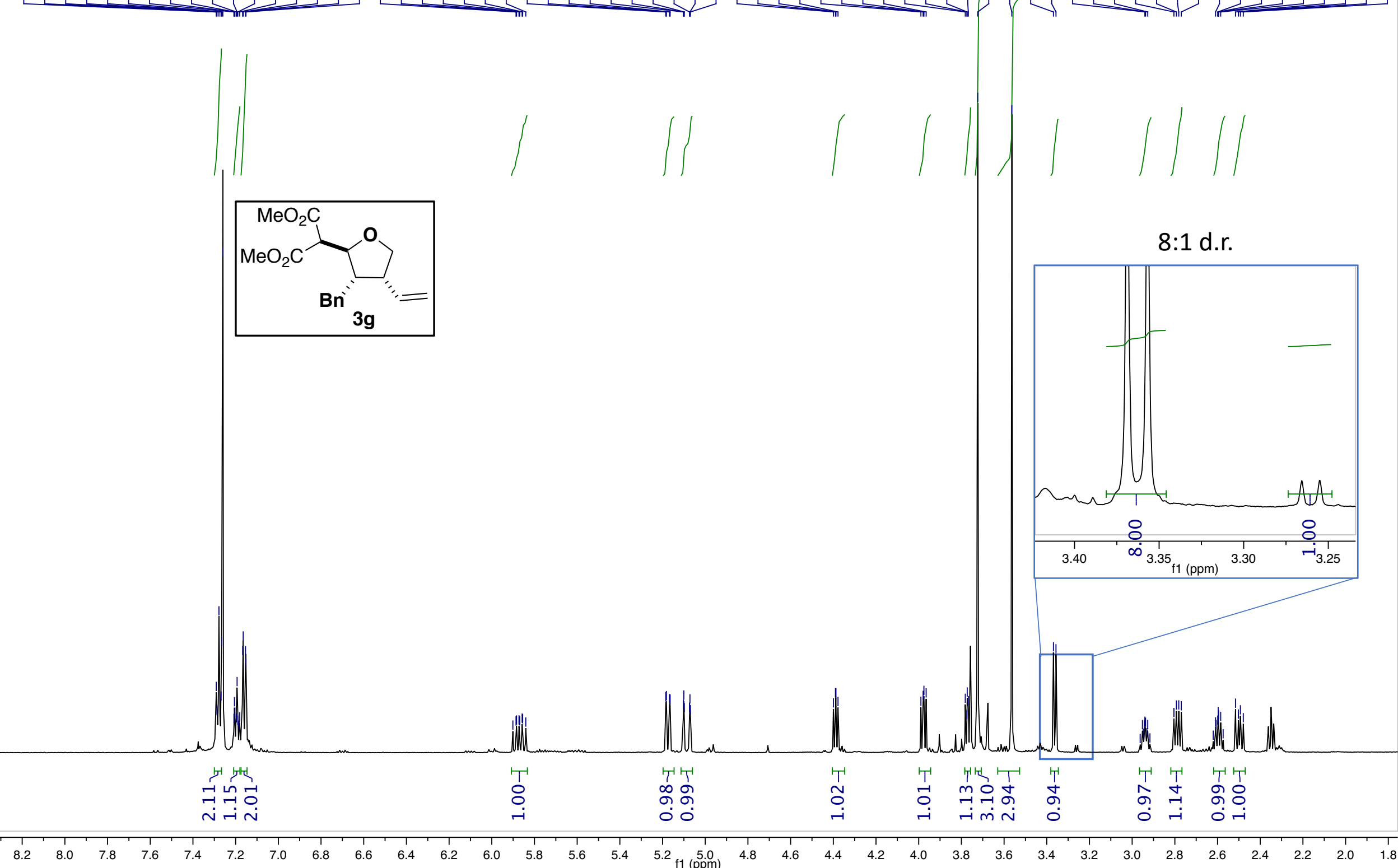




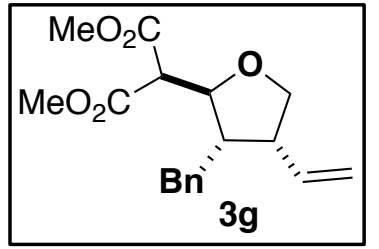

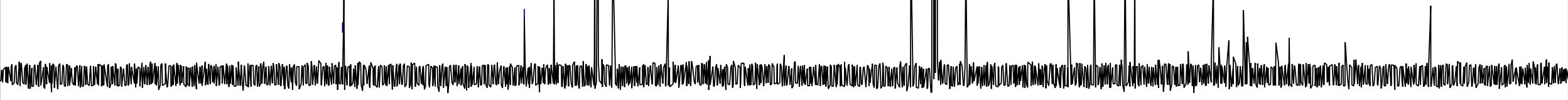

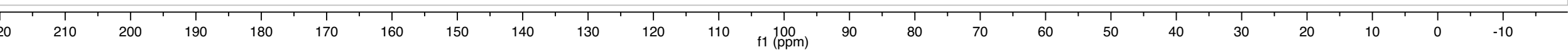


$\frac{m}{\mathrm{U}}$

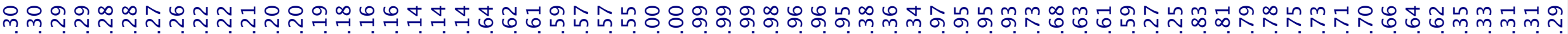

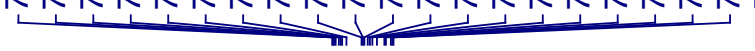

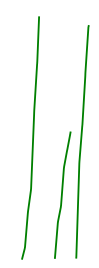

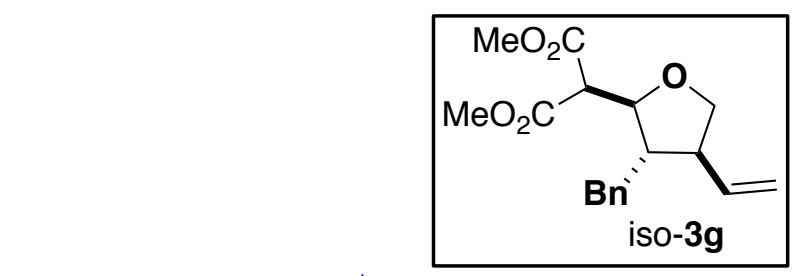

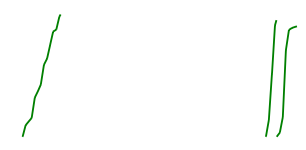

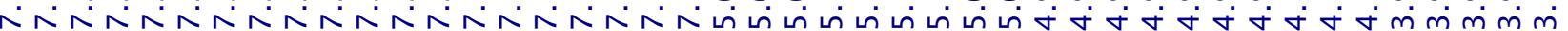

- 1 inl
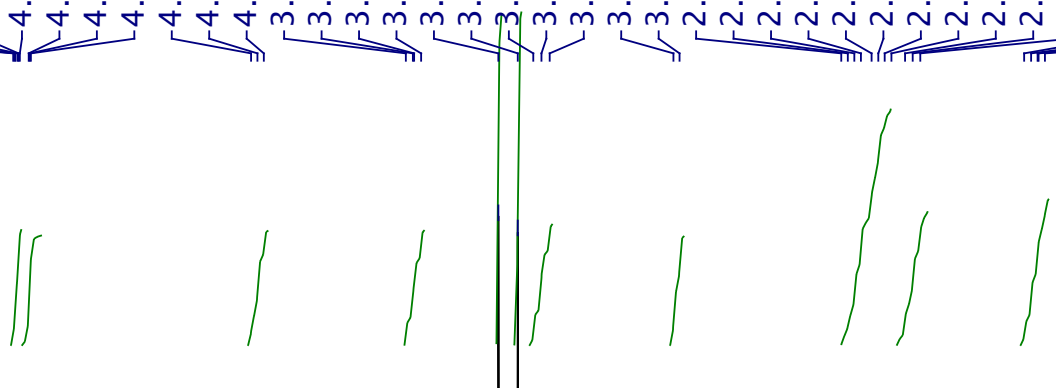

$14: 1$ d.r.
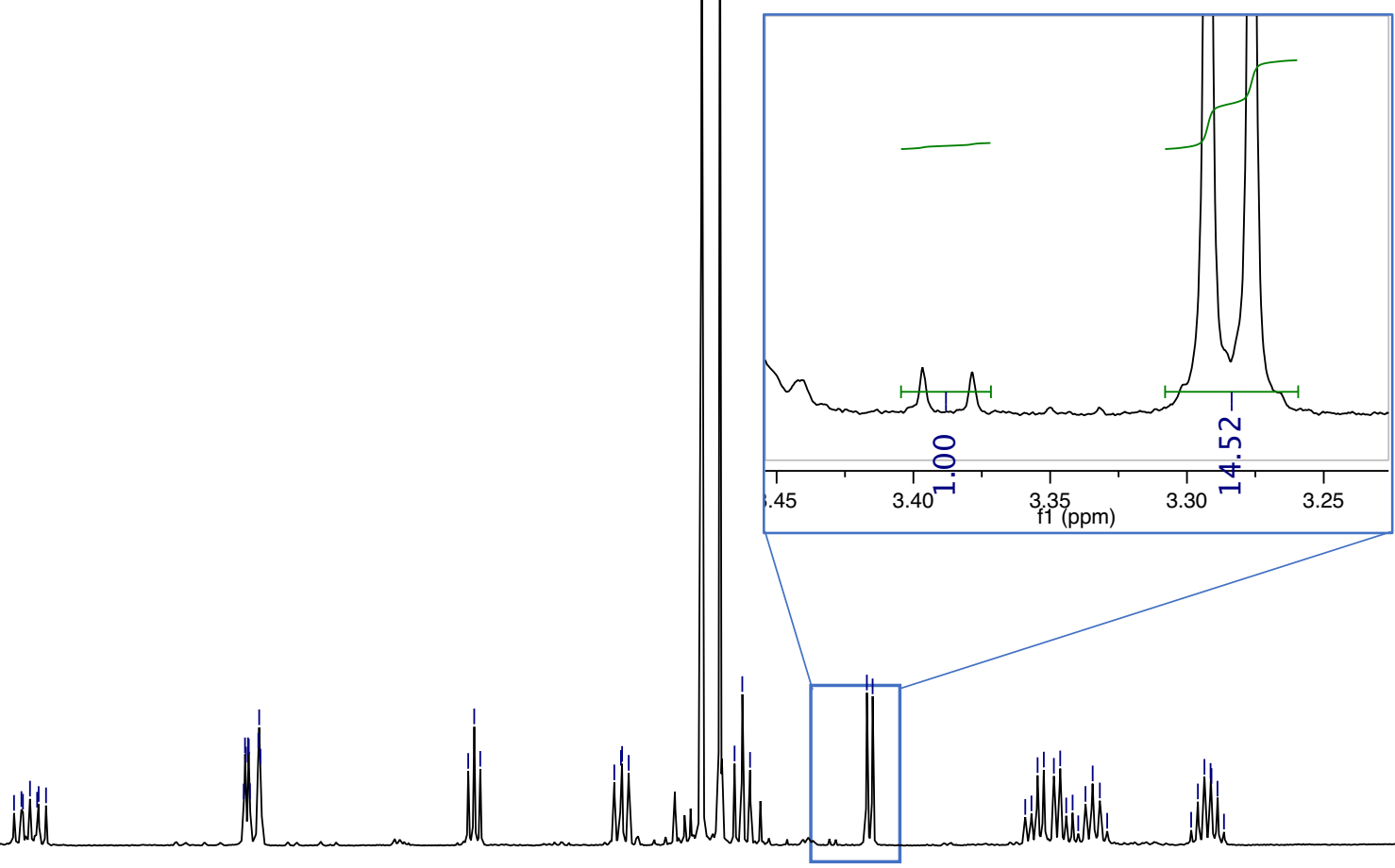

$\stackrel{T}{ت}$

늑

$\sim \sim \sim \sim \sim$

. 


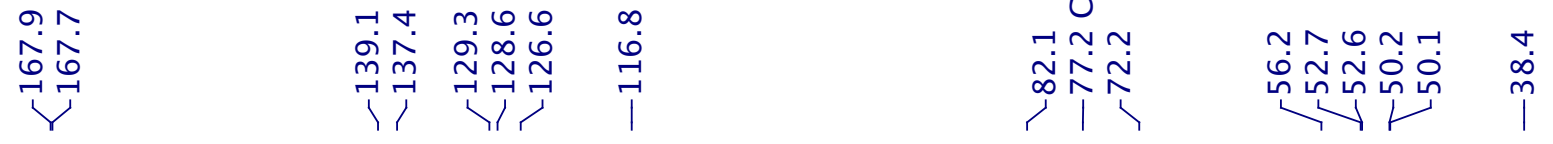

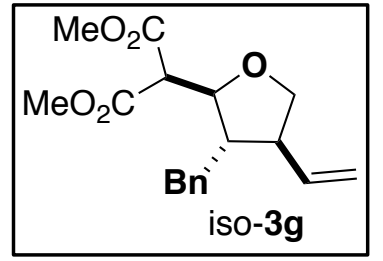

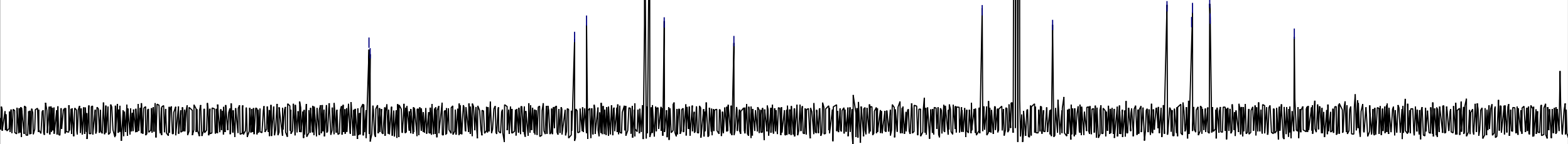

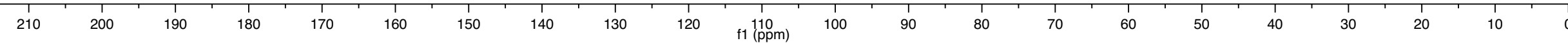


บั

$m$ m

iñNiñ
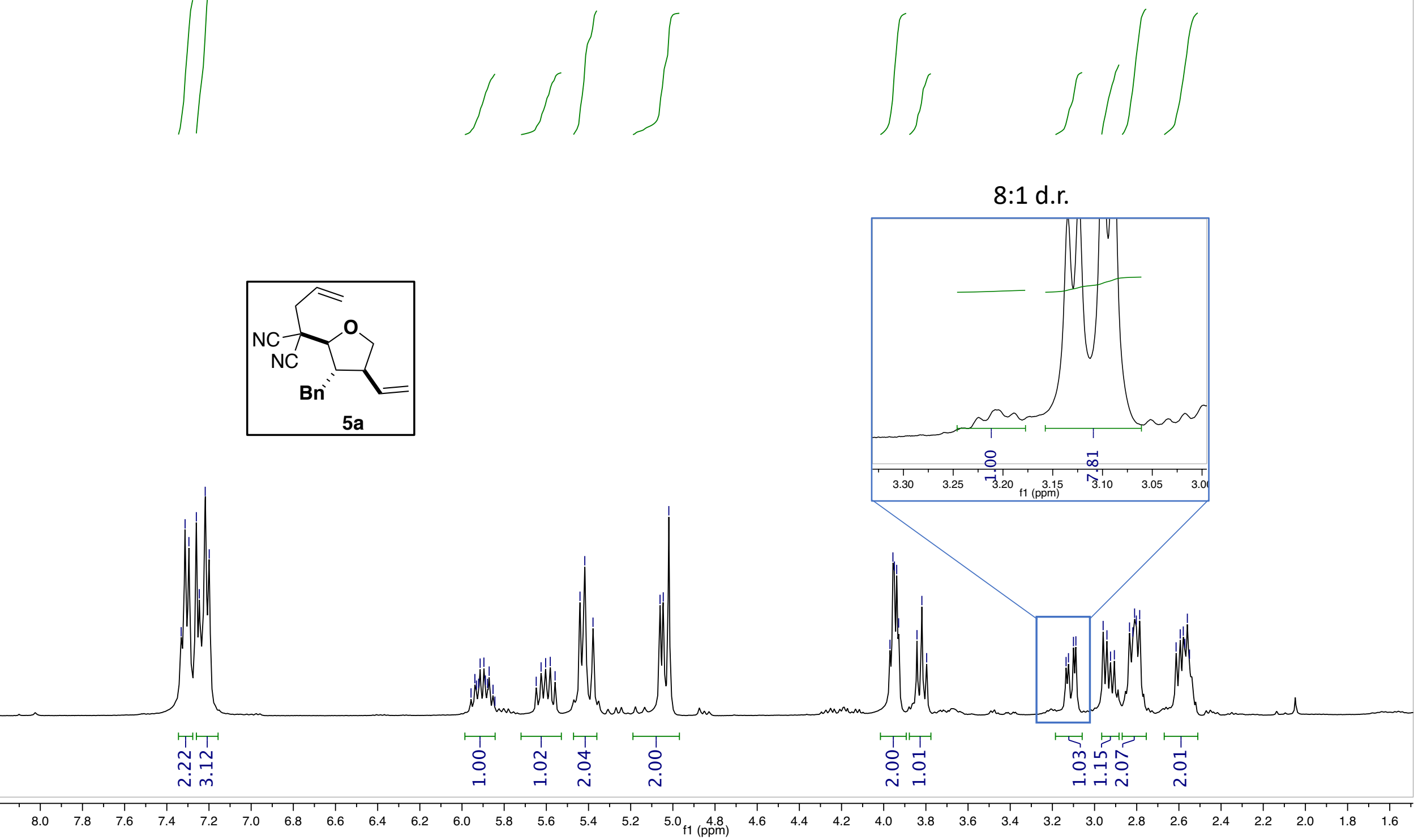

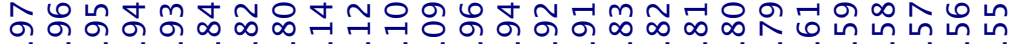

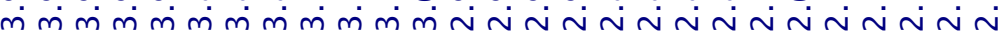
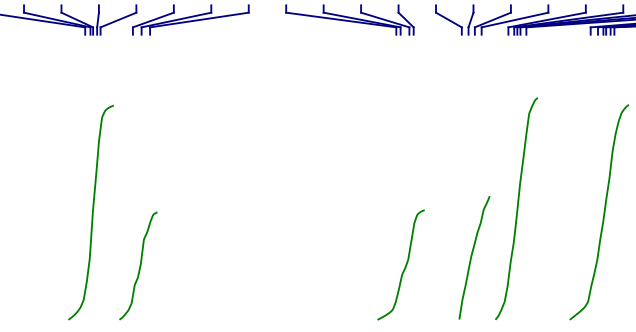

8:1 d.r.

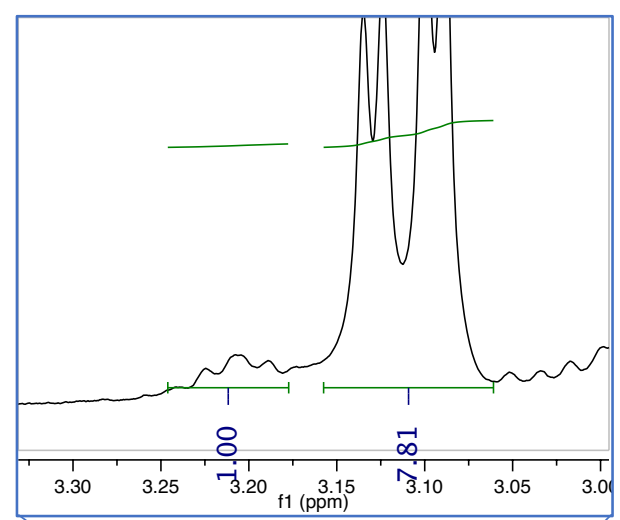




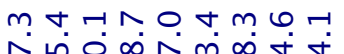

m $m \underset{\sim}{m} \stackrel{\infty}{\sim} \underset{\sim}{\sim} \stackrel{\sim}{\sim} \underset{\sim}{+}$

रा।
บั

$m \wedge m$

$1 \backslash 1$ mogom

윰궁ํํ

।

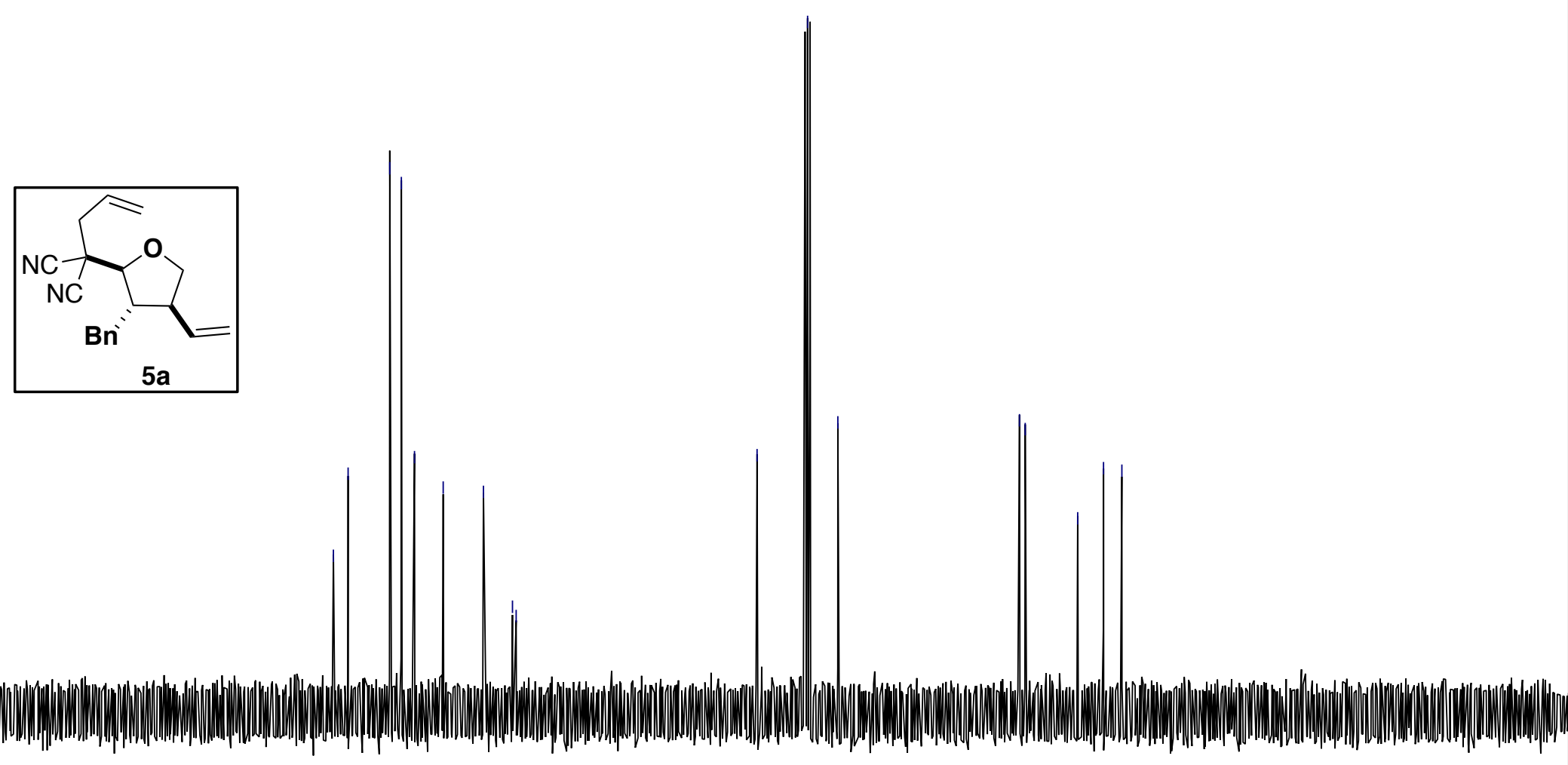


产

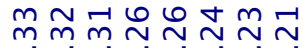

NNNNNNN

un th $\ln$ in th

(1)
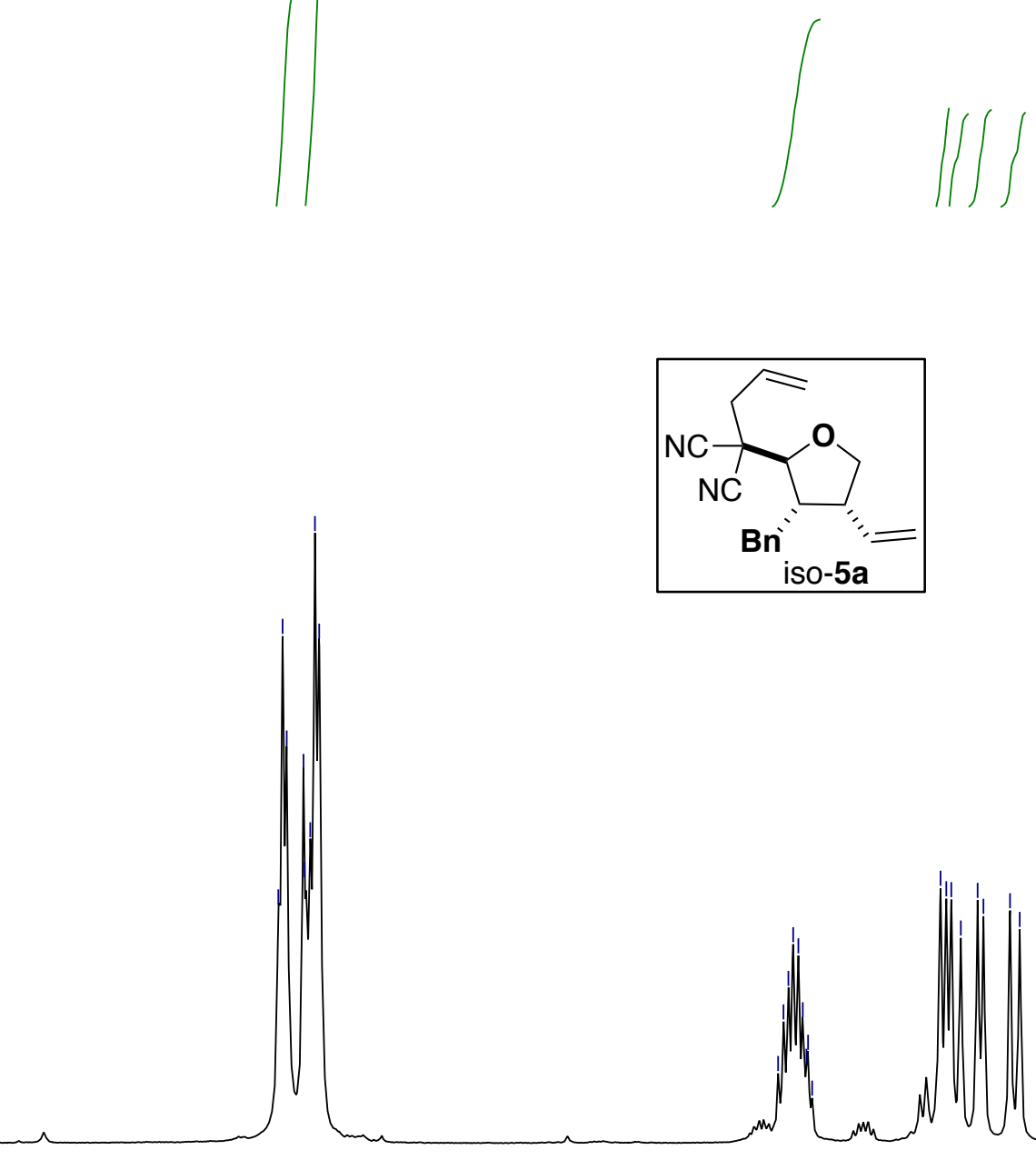

T'T

$\stackrel{m}{\sim}$

in

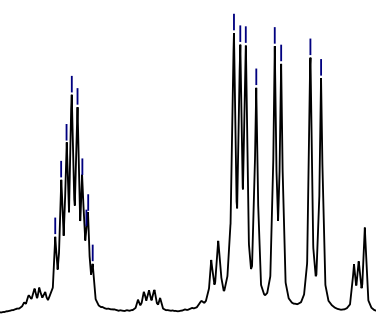

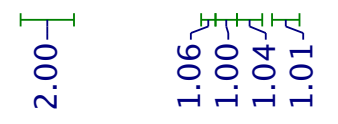

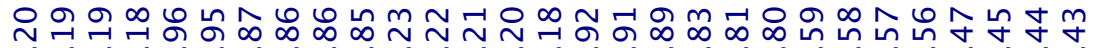

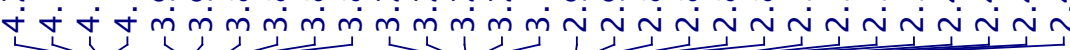
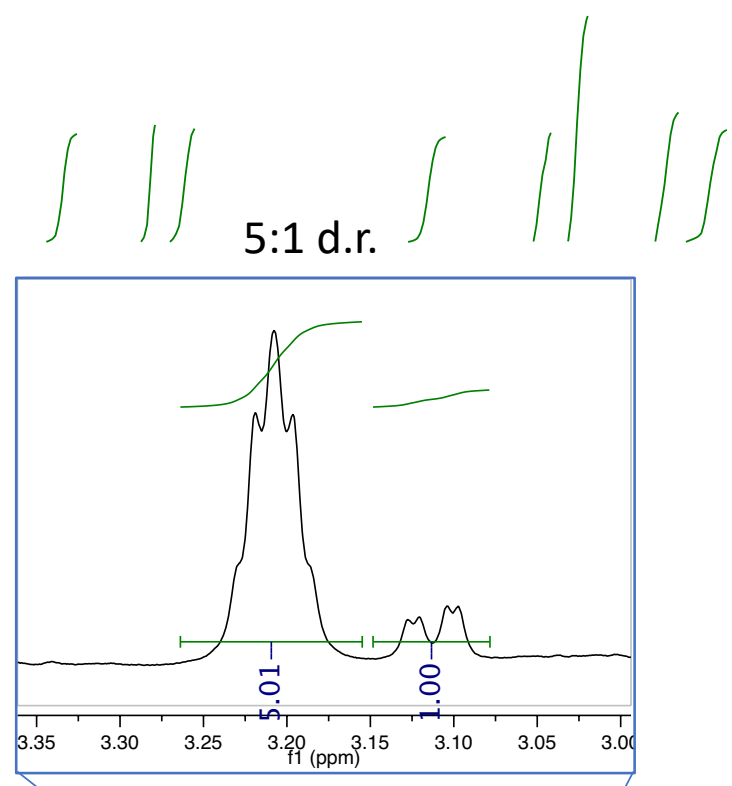
thent
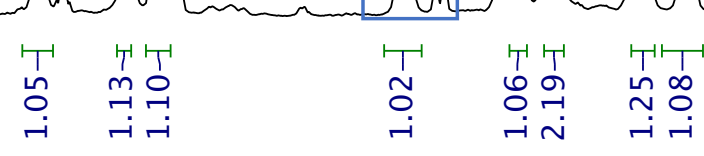

8.0

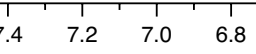

$\begin{array}{llll}8 & 6.6 & 6.4 & 6.2\end{array}$

$6.0 \quad 5.8 \quad 5$

$\begin{array}{ll}5.4 & 5.2\end{array}$

$5.0 \quad \stackrel{4.8}{\mathrm{fpm})}$

3.6

3.23.

$1+$ 

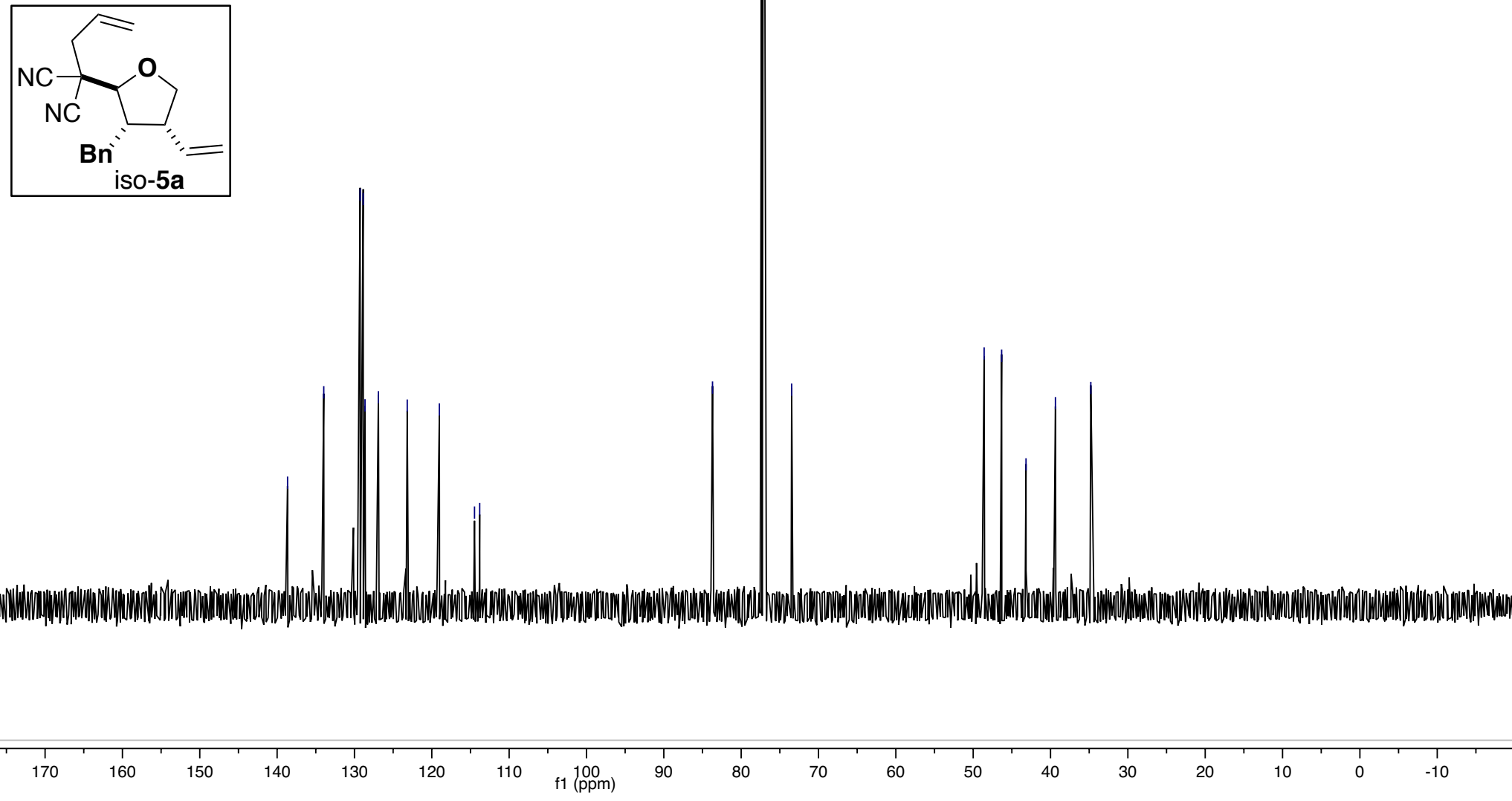


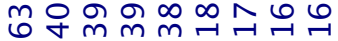

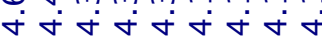

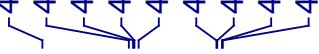

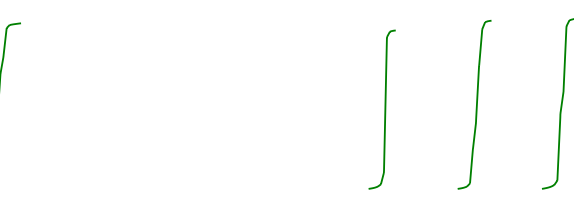

긔걱ㄱㅇㅇㅇㅇㅇ mं mं mंm

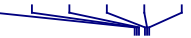

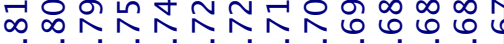
ninninninininu
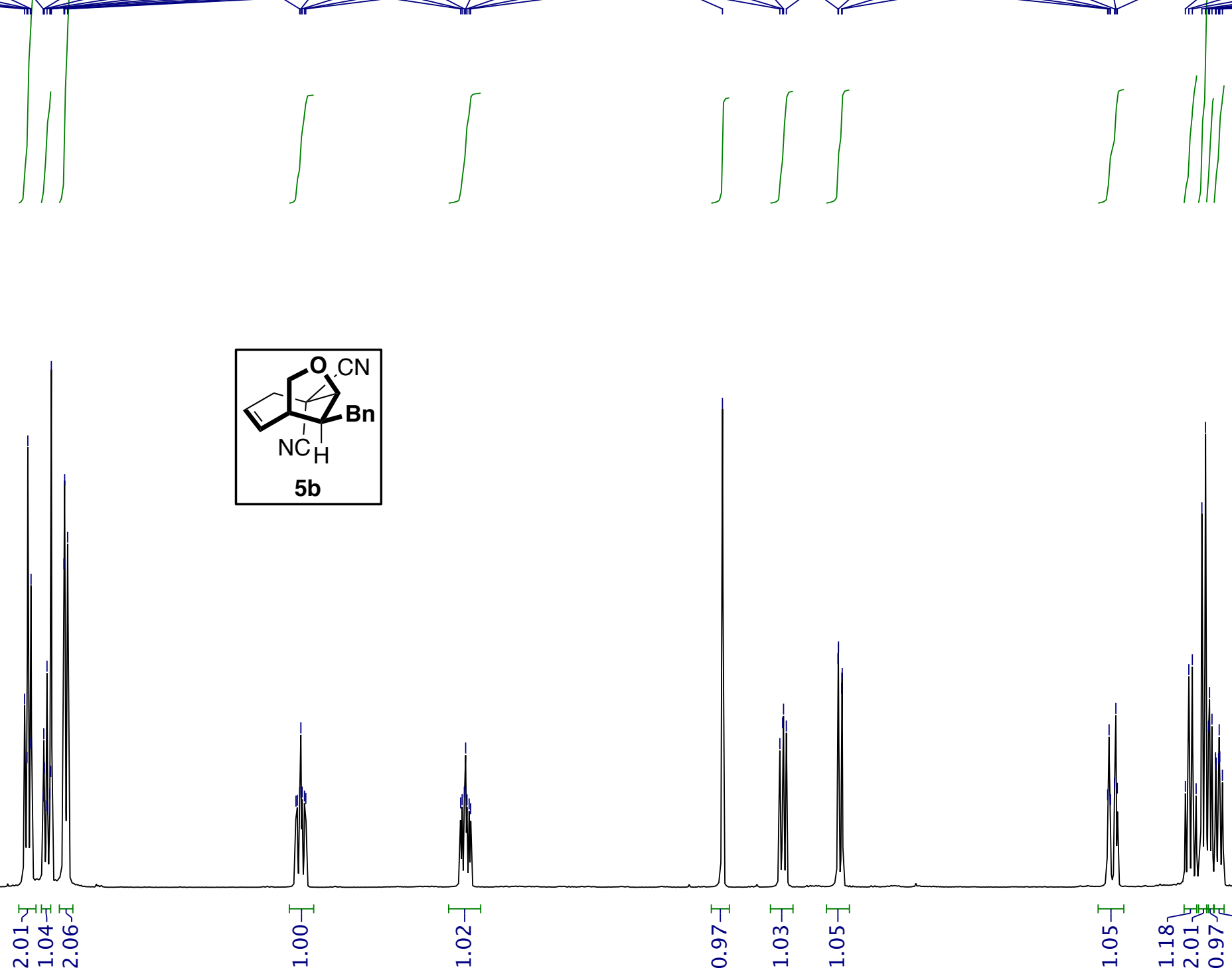

$\begin{array}{ccc}T & T & T \\ \hat{a} & m & 0 \\ 0 & 0 & 0 \\ 0 & -1 & -i\end{array}$

T कायू

놈 कनतेळ

5.5

5.0

f1 (ppm)

4.5

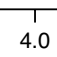




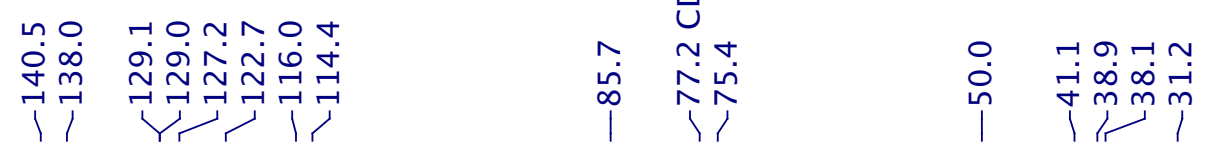
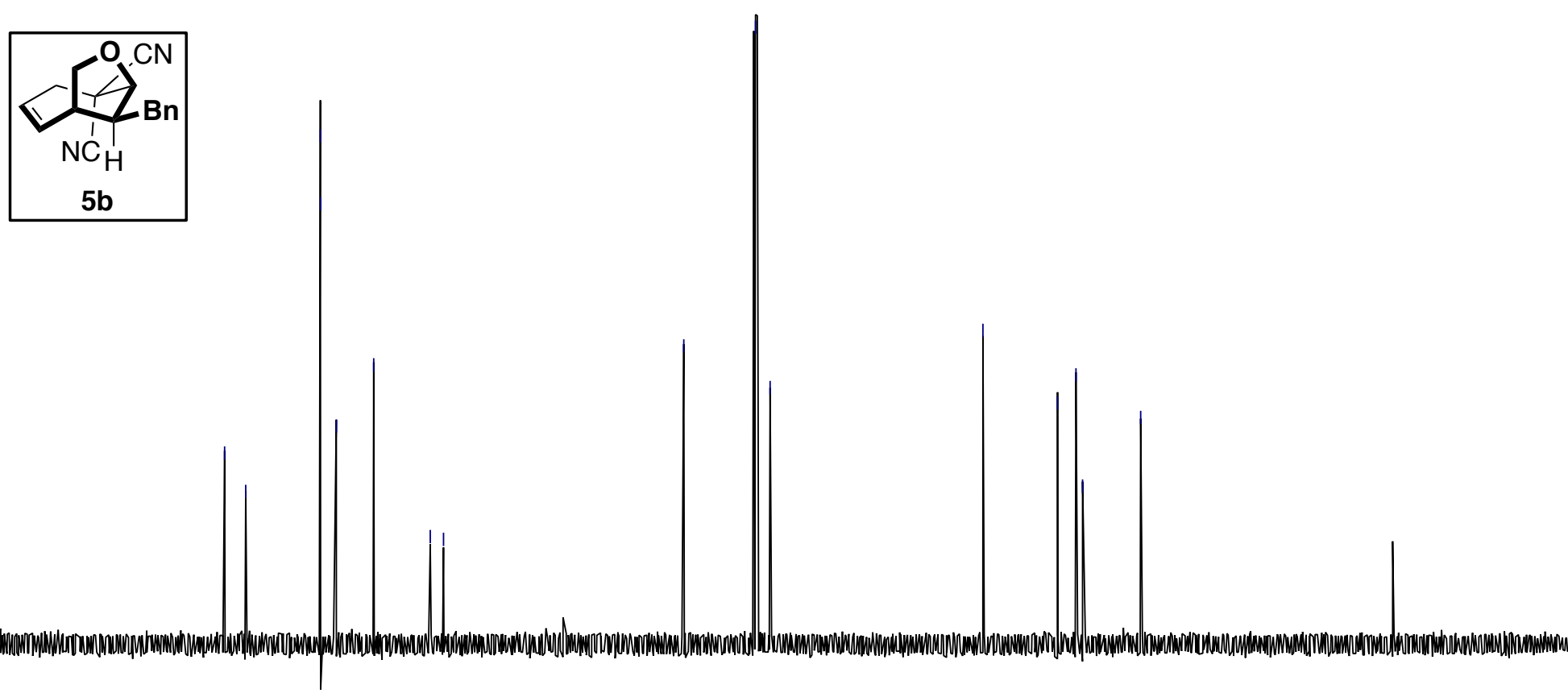


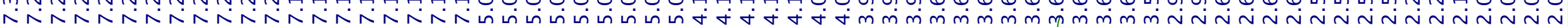

गा1
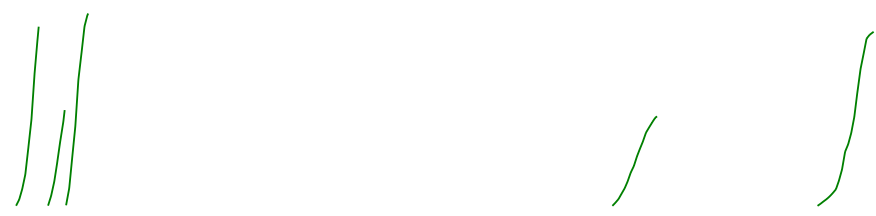
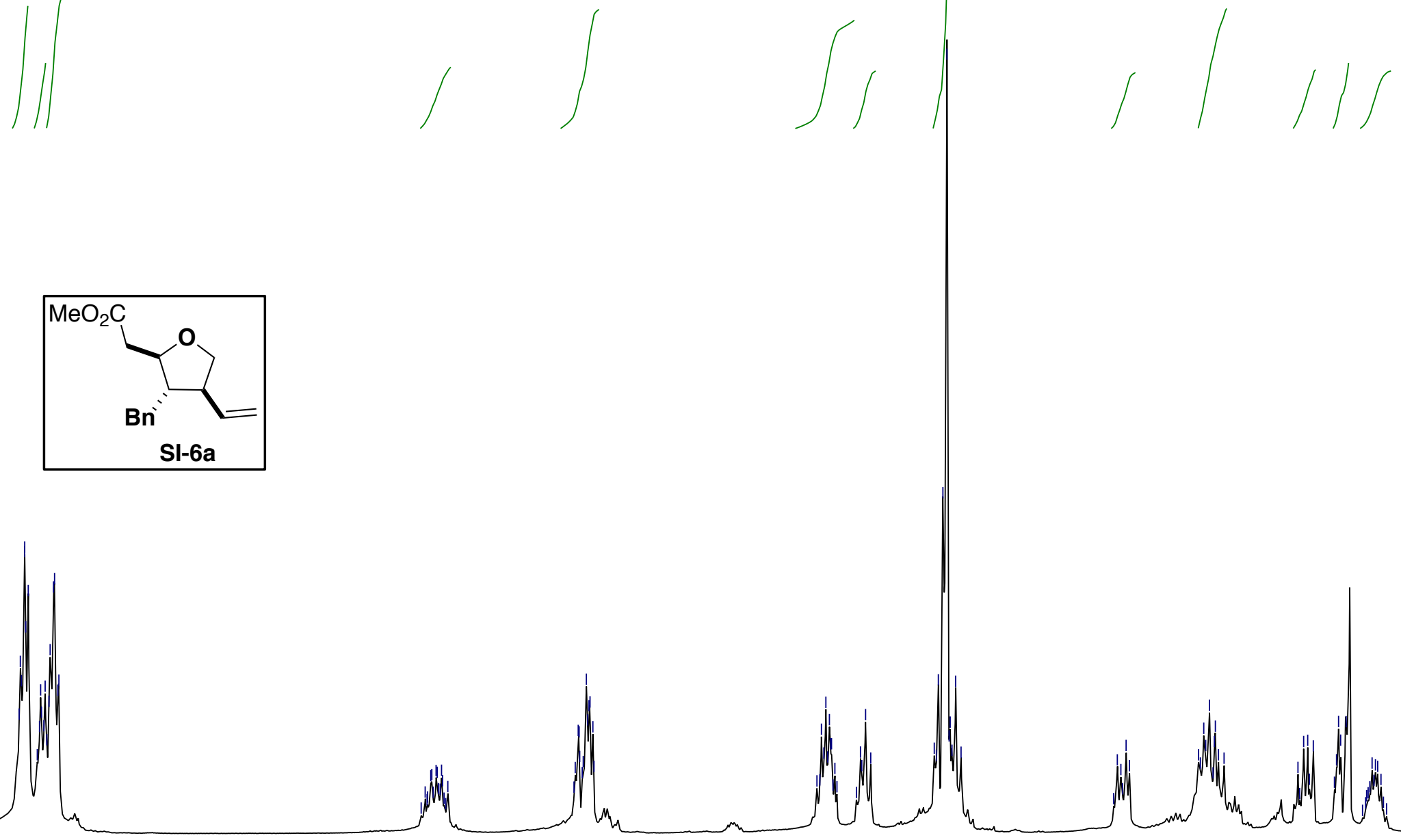

श्राप्र

คำ

ก

特

คำ

$I_{m}$

$\stackrel{m}{\sim}$

ก

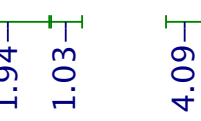

$0_{\mathrm{f} 1}^{4}(\mathrm{ppm})$

$4.0 \quad 3.8$

3.6

4.2

(1)

2.6

2.4

(11) 


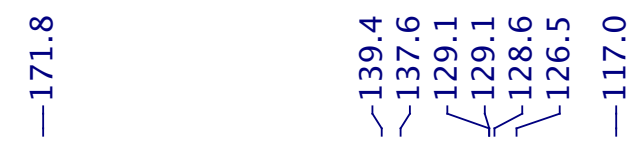

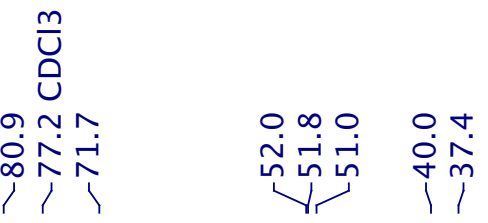

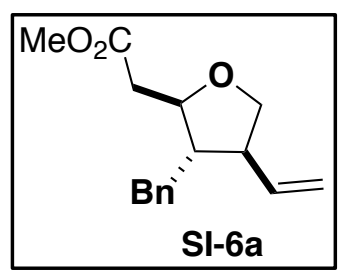


高

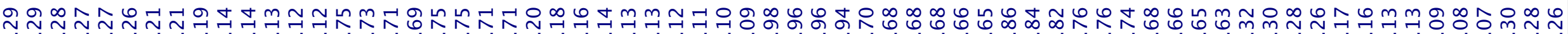

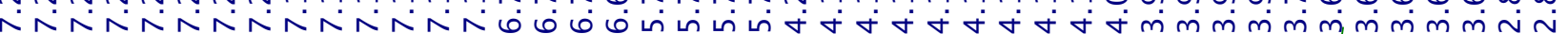

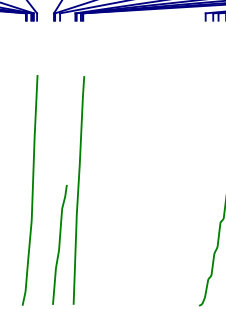

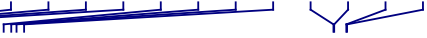
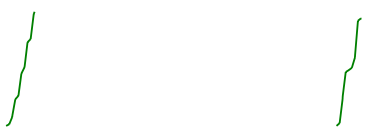
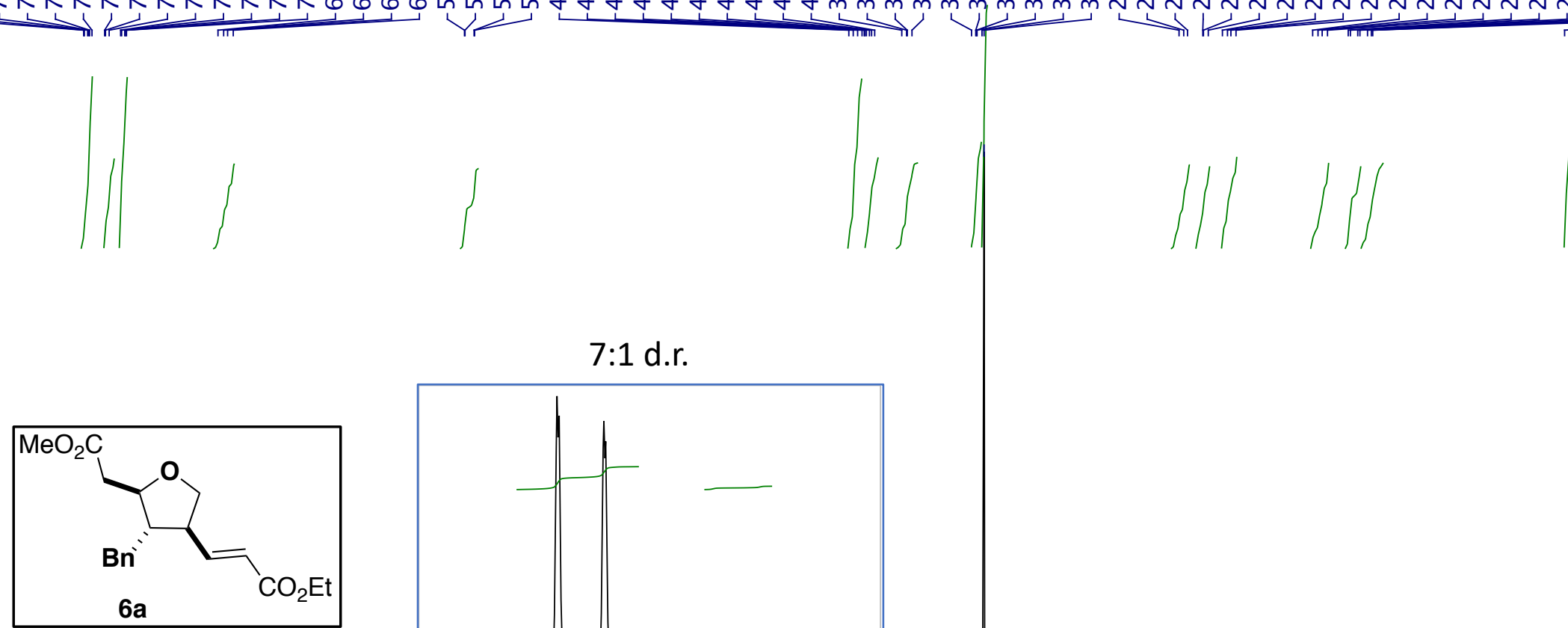

\section{7:1 d.r.}
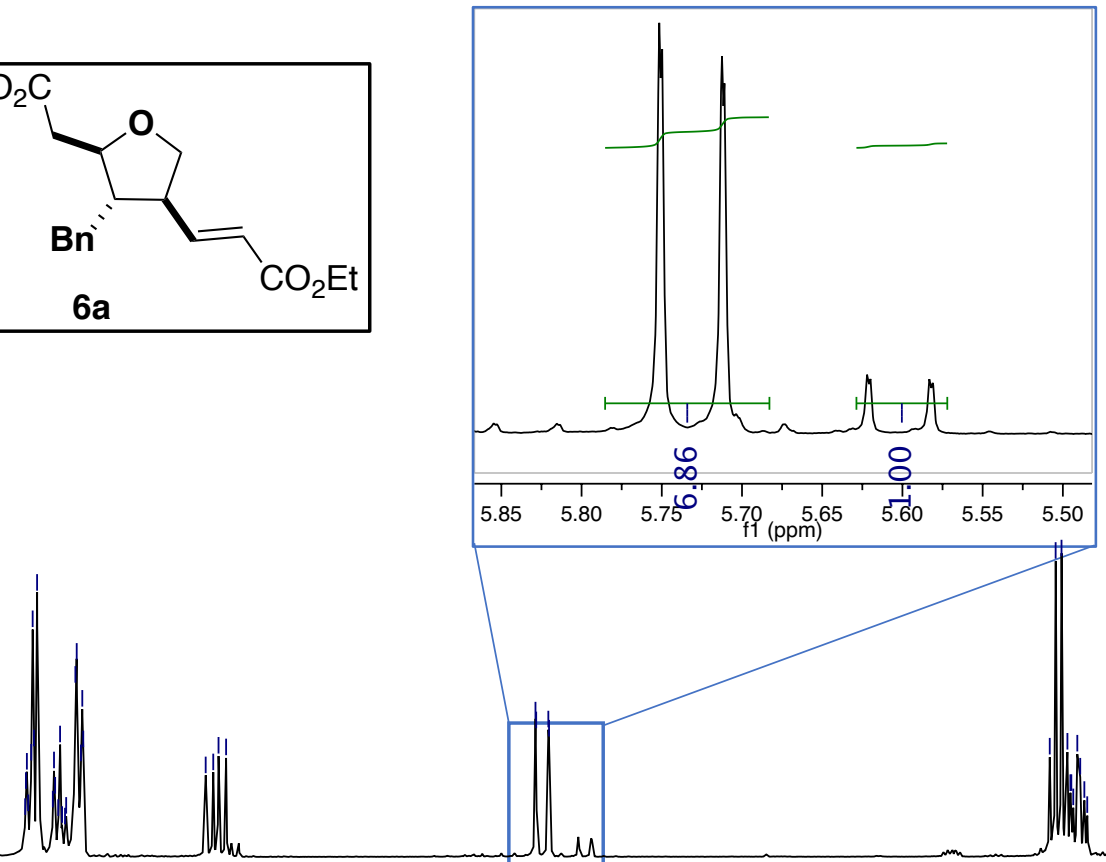

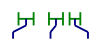
는 $\underset{-1}{\sim}$ $\sim \ddot{\sim} \sim$

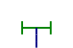

1
0 -i 


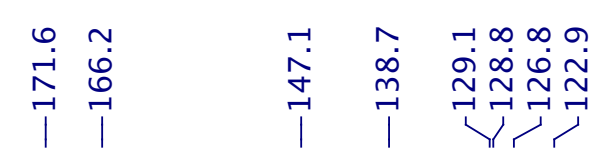

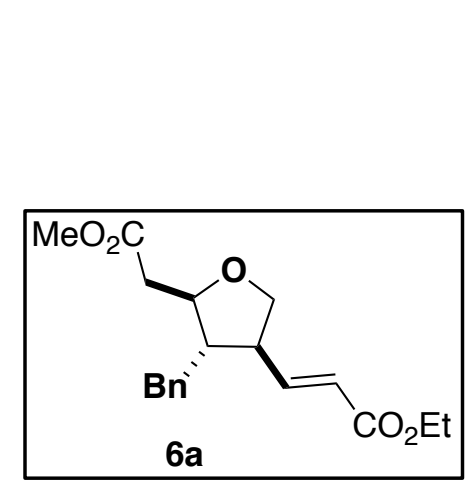




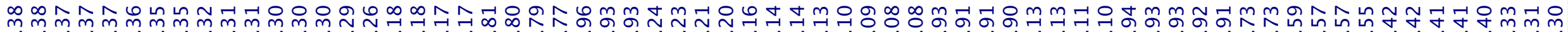

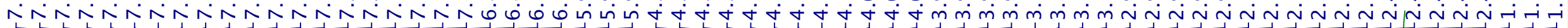
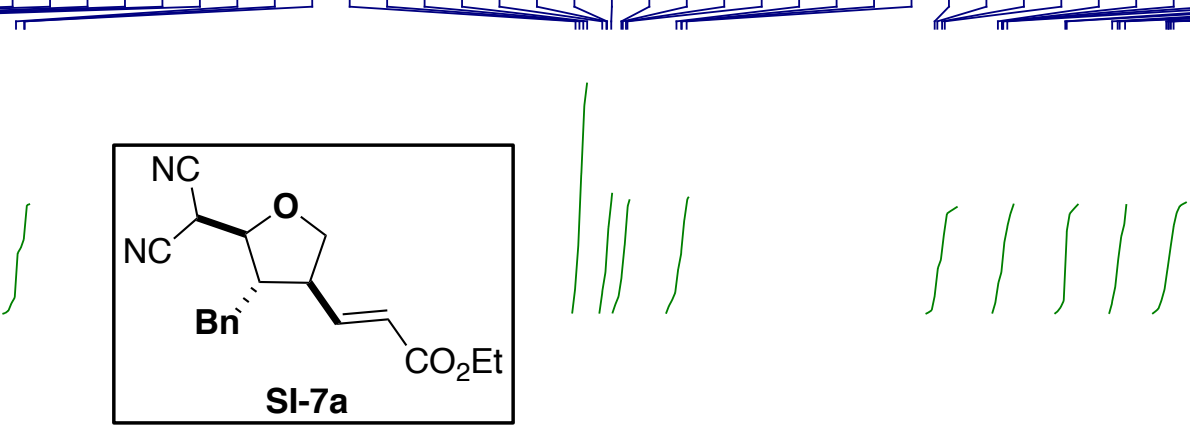

6:1 d.r.
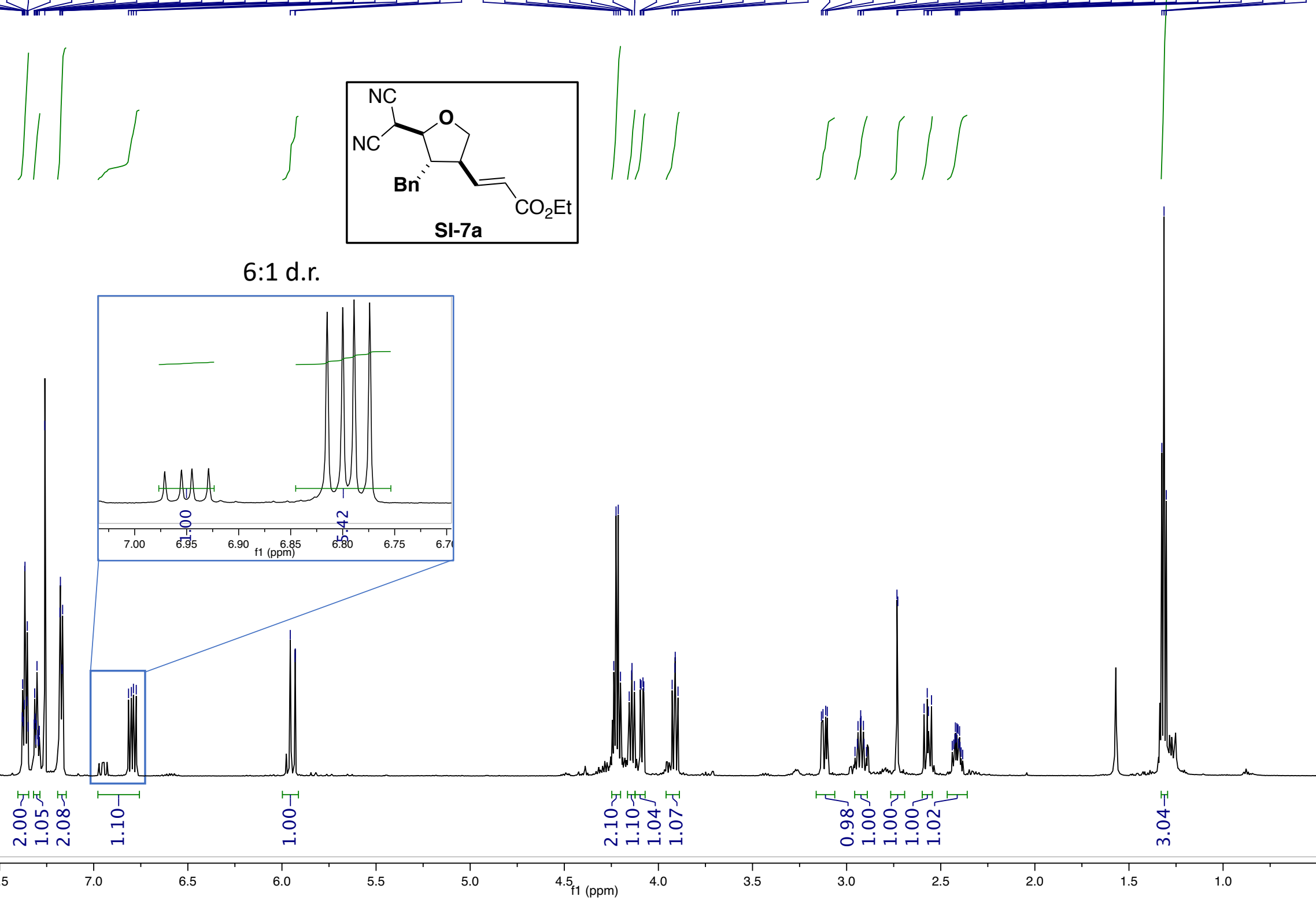

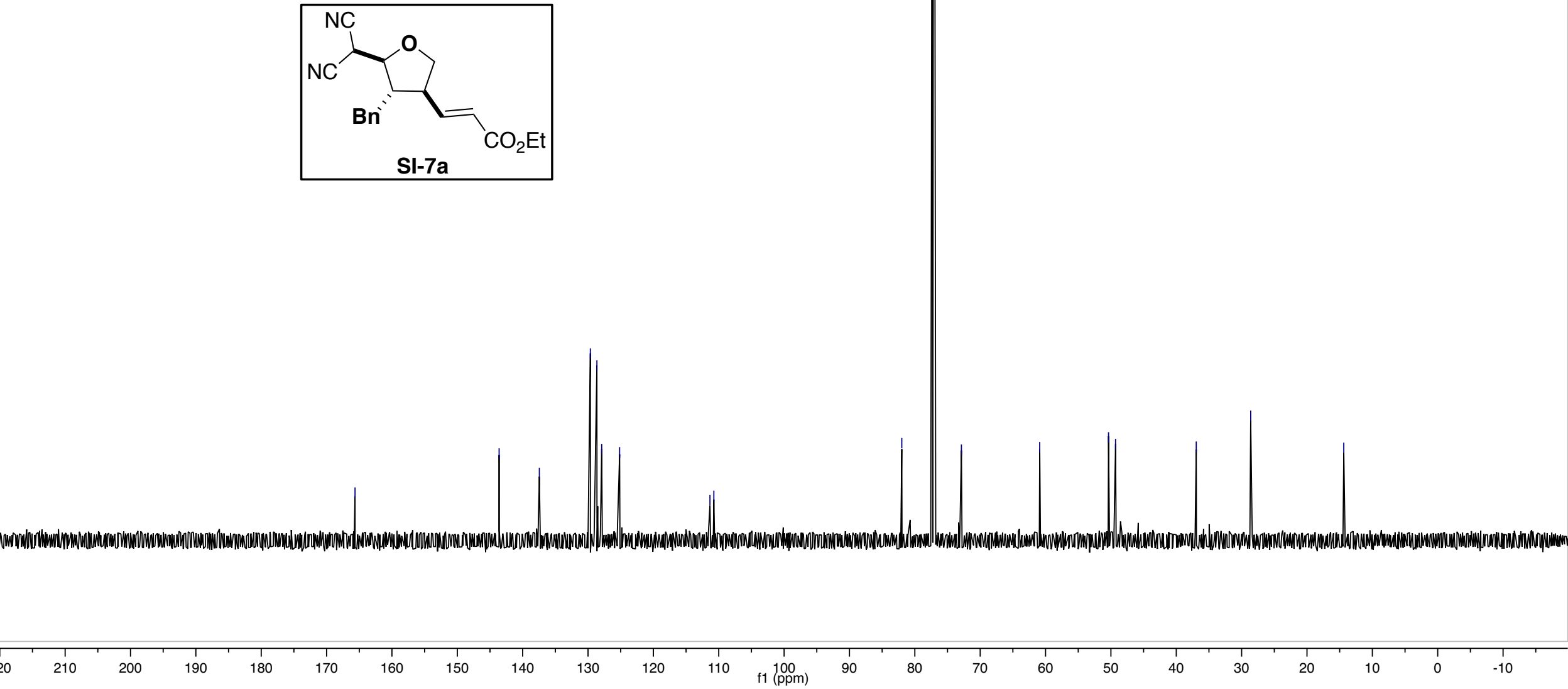
บับ

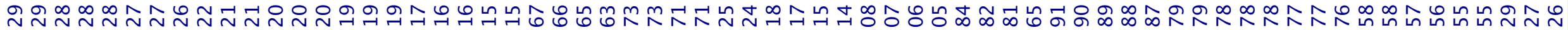

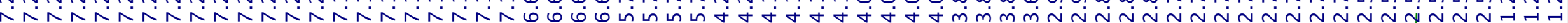
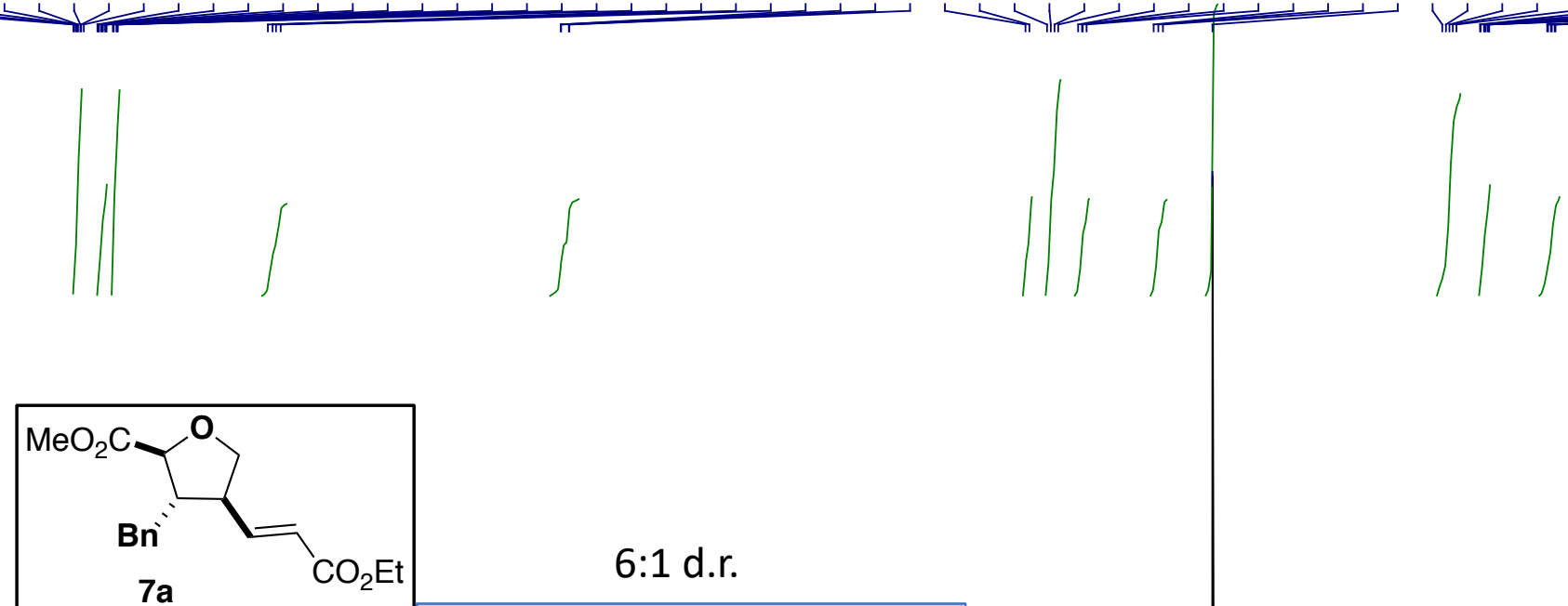

6:1 d.r.
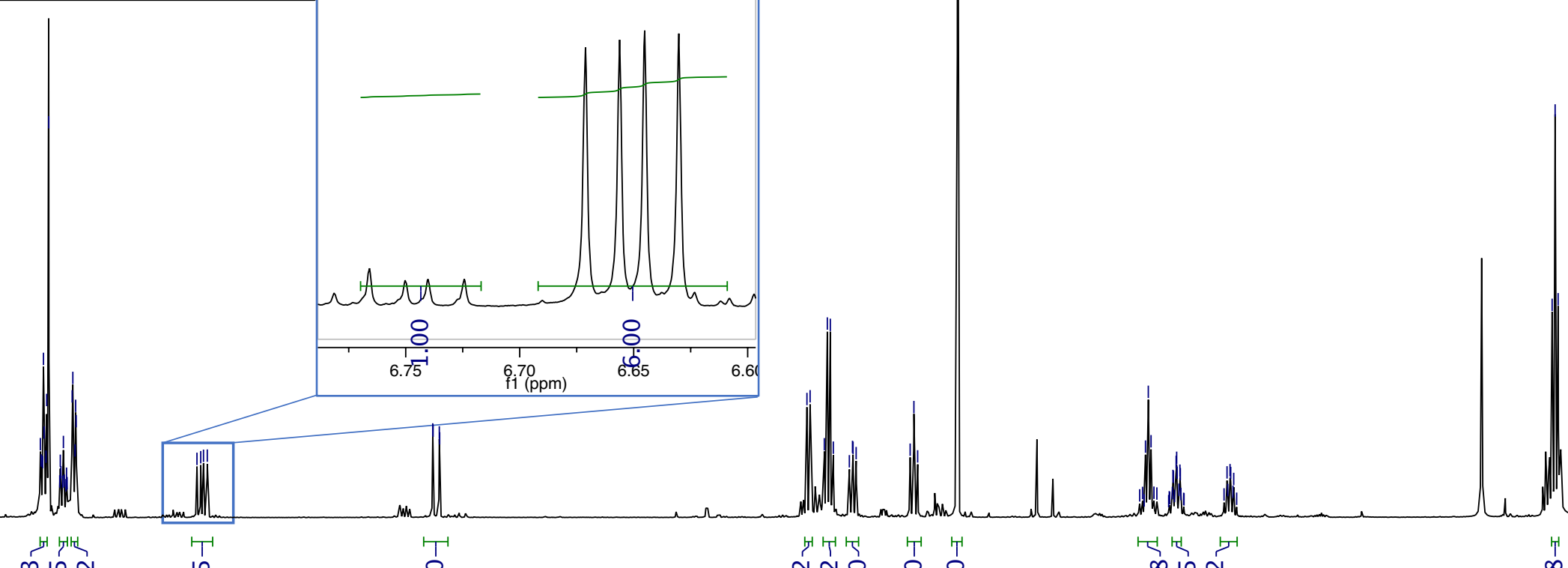

$m$ 늠

กี่

กิ
ตุ

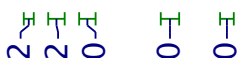

증 응

प्रे

능 중

Ni

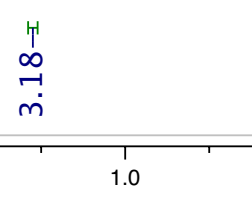




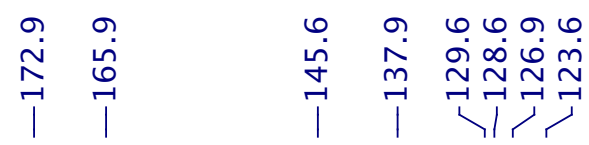

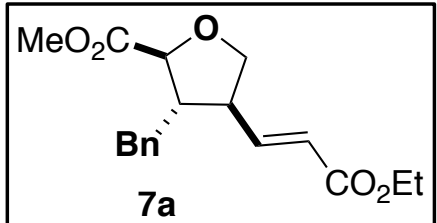




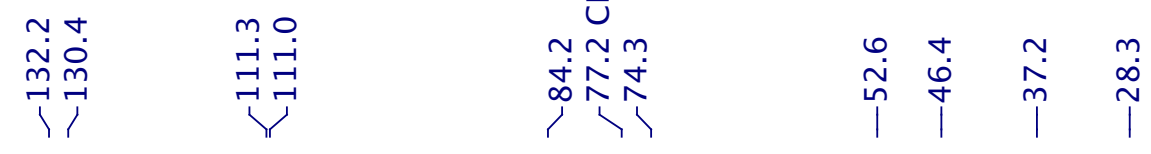

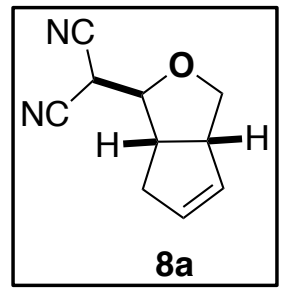

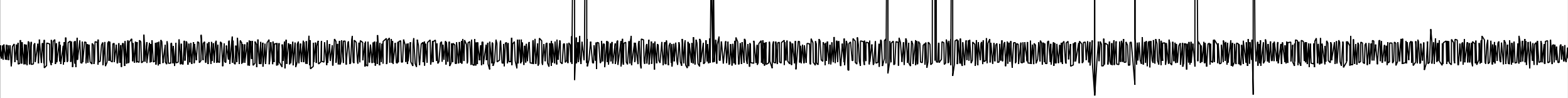

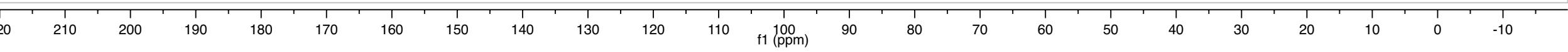


(1)
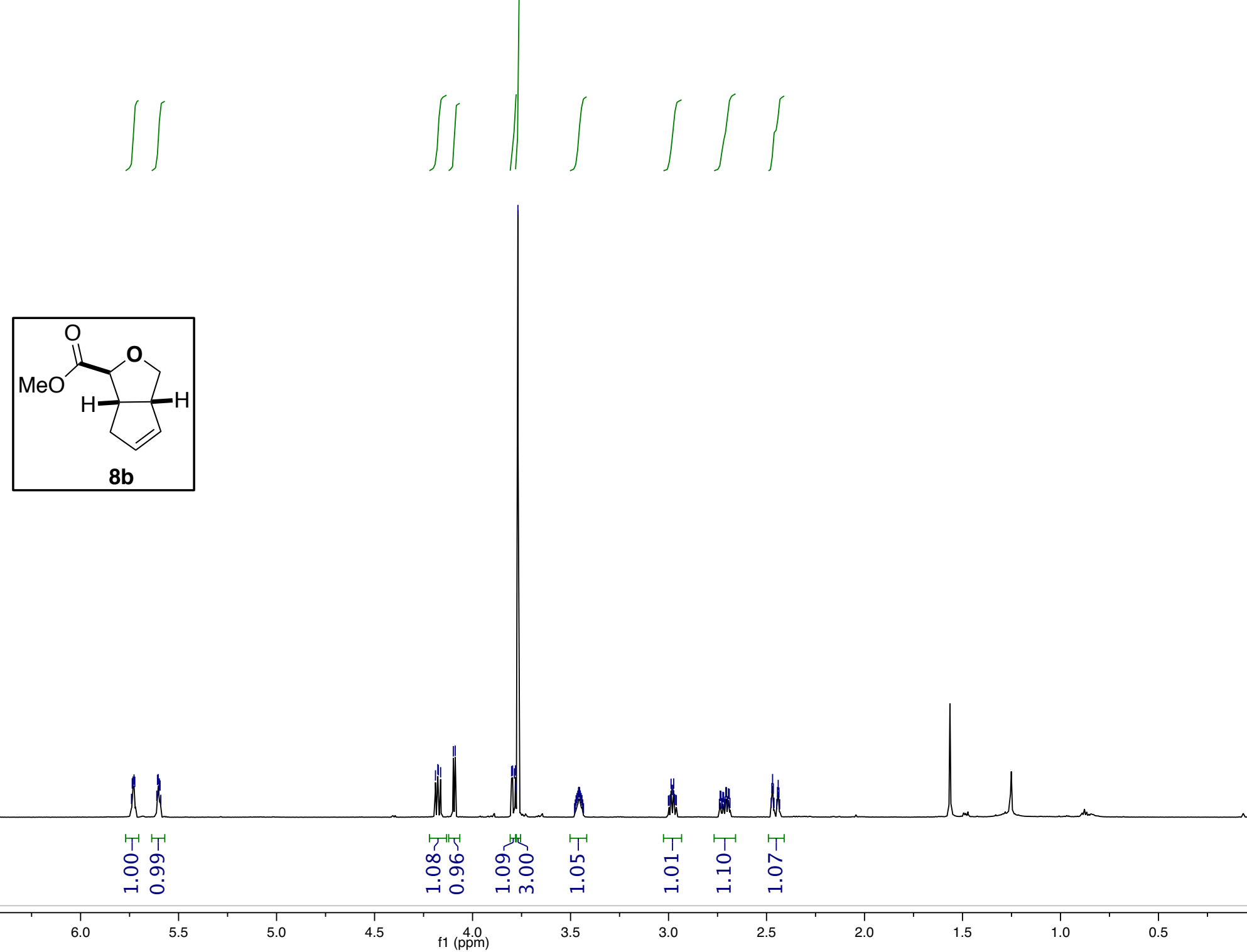


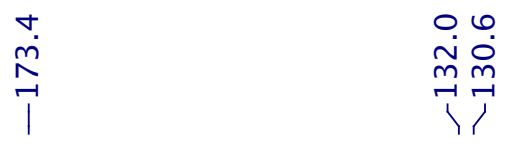

出

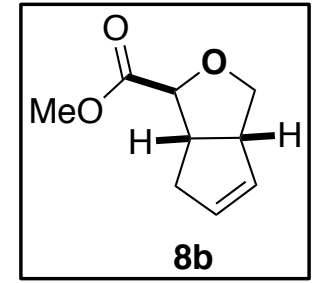

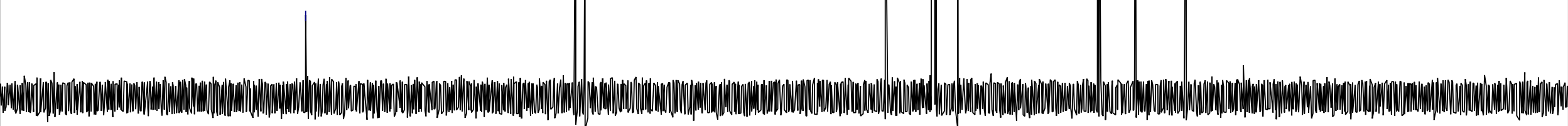
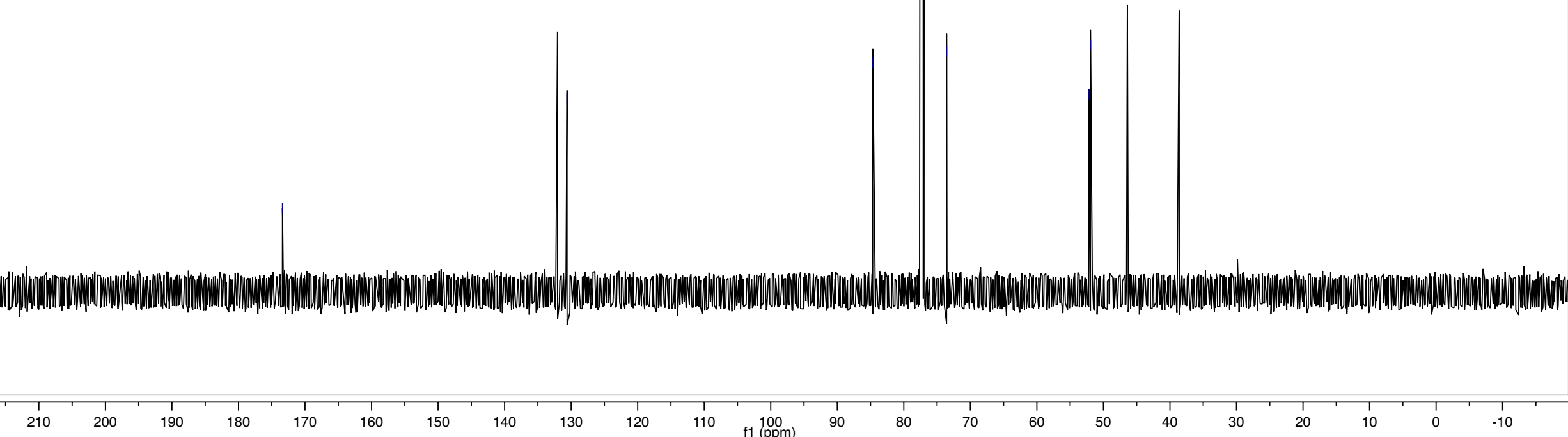


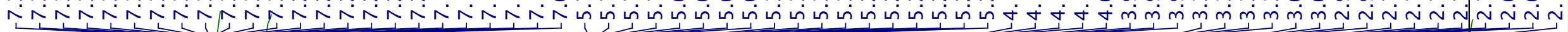
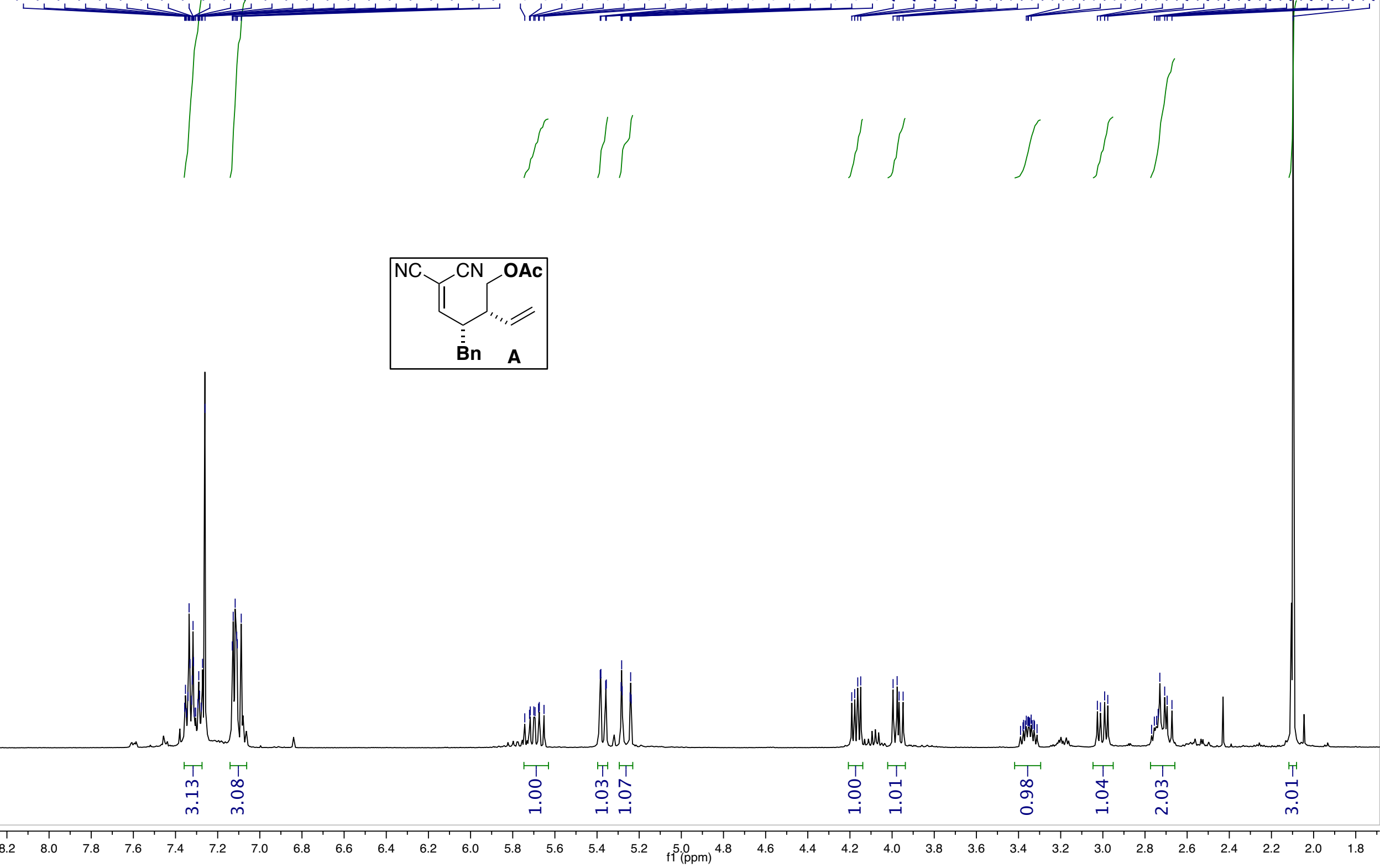


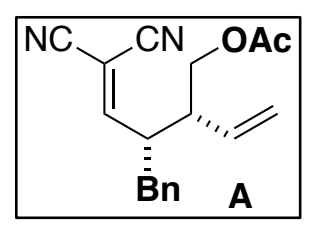

